\title{
BROOKHFUEN
}

NATIONAL LABORATORY

BNL-77900-2007-IR

\section{High-Performance Coating Materials}

\author{
Toshifumi Sugama
}

July 2006

\section{Energy Sciences and Technology Department/Energy Resources Division Brookhaven National Laboratory P.O. Box 5000 \\ Upton, NY 11973-5000 www.bnl.gov}

Notice: This manuscript has been authored by employees of Brookhaven Science Associates, LLC under Contract No. DE-AC02-98CH10886 with the U.S. Department of Energy. The publisher by accepting the manuscript for publication acknowledges that the United States Government retains a non-exclusive, paid-up, irrevocable, world-wide license to publish or reproduce the published form of this manuscript, or allow others to do so, for United States Government purposes. 


\section{DISCLAIMER}

This report was prepared as an account of work sponsored by an agency of the United States Government. Neither the United States Government nor any agency thereof, nor any of their employees, nor any of their contractors, subcontractors, or their employees, makes any warranty, express or implied, or assumes any legal liability or responsibility for the accuracy, completeness, or any third party's use or the results of such use of any information, apparatus, product, or process disclosed, or represents that its use would not infringe privately owned rights. Reference herein to any specific commercial product, process, or service by trade name, trademark, manufacturer, or otherwise, does not necessarily constitute or imply its endorsement, recommendation, or favoring by the United States Government or any agency thereof or its contractors or subcontractors. The views and opinions of authors expressed herein do not necessarily state or reflect those of the United States Government or any agency thereof. 


\title{
High-performance Coating Materials
}

\author{
Final Report
}

July 2006

Prepared for

The U.S. Department of Energies Office of Geothermal Technologies

1000 Independence Avenue SW

Washington, D.C. 20585-0121

Prepared by

Toshifumi Sugama

Energy Resources Division

Energy Science \& Technology Department

Brookhaven National Laboratory

Upton, NY 11973

This manuscript, issued by DOE Office of Geothermal Technologies, has been authored by Brookhaven Science Associates, LLC under Contract No. DE-AC02-98CHI-886 with the U.S. Department of Energy. The United States Government retains, and publisher, by accepting the article for publication, acknowledges, a world-wide license to publish or reproduce the published form of this manuscript, or allow others to do so, for the United States Government purposes. 


\section{TABLE OF CONTENTS}

$\begin{array}{ll}\text { Summary } & \text { Pages } \\ \text { Collaborators } & 6 \\ \text { 1. Introduction } & 14 \\ \text { 2. Coatings for HX Tubes } & 15 \\ \text { 2.1. Adherence to Underlying Steel } & 18 \\ \quad \text { 2.1.1. Experimental } & 19 \\ \text { 2.1.2. Results } & 20\end{array}$

2.1.2.1. Bond Mechanisms at Interfaces Between PPS and $\begin{array}{ll}\mathrm{ZnPh} & 21\end{array}$

2.1.2.2. Cathodic Delamination 25

2.2. Thermal Conductivity and Mechanical Properties 28

2.2.1. Experimental 29

2.2.2. Results 29

2.3. Corrosion-preventing Performance 32

2.3.1. Experimental 33

2.3.2. Results 34

2.4. Anti-hydrothermal Oxidation 35

2.4.1. Experimental 36

2.4.2. Results 36

2.4.2.1. Thermodynamic Properties 36

2.4.2.2. Surface Chemistry 38

2.4.2.3. Inertness to Silica Scale Deposition 40

2.5. Resistance to Abrasive Wear 43

2.5.1. Experimental 44

2.5.2. Results 44

2.6. Self-repairing Property 46

2.6.1. Experimental $\quad 47$

2.6.2. Results 48

2.6.2.1. SEM-EDX Analyses 48 
2.6.2.2. EIS Measurement 50

2.6.2.3. XRD Analysis $\quad 52$

2.7. Field Validation Tests

2.7.1. Full-scale Lining Technologies 55

2.7.2. Two-year Long Field Exposure Test at Mammoth

Power Plant 62

2.7.2.1. Experimental 63

2.7.2.2. Results 64

2.7.2.2.1. Scale Deposition 64

2.7.2.2.2. Liner’s Surfaces 69

2.7.3. Field Test at Puna Power Plant 70

$\begin{array}{ll}\text { 2.7.3.1. Results } & 71\end{array}$

$\begin{array}{ll}\text { 2.8. Conclusions } & 77\end{array}$

2.9. Technology Transfer 82

2.10. Awards 82

2.11. Publications $\quad 82$

2.12. Conf. Proceedings 83

3. Coatings for HX Tube/Sheet and Pipe/Pipe Joint Areas 84

3.1. PTFHFP Coating $\quad 84$

3.1.1. Experimental $\quad 84$

3.1.2, Results 85

3.2. PBI Coating 86

3.2.1. Experimental $\quad 86$

$\begin{array}{ll}\text { 3.2.2. Results } & 87\end{array}$

$\begin{array}{ll}\text { 3.3. PES Coating } & 87\end{array}$

3.3.1. Experimental $\quad 88$

3.3.2. Results 88

3.4. CAC-filled SAL Coating 89

3.4.1. Experimental $\quad 90$

$\begin{array}{ll}\text { 3.4.2. Results } & 91\end{array}$

3.5. Three-mo. Long Field Exposure Tests 93 
3.6. Conclusions 93

3.7. Publications 95

4. Coatings for Wellhead Components 95

4.1. CVD-Titanium Carbonitride 95

4.1.1. Experimental 96

$\begin{array}{ll}\text { 4.1.2. Results } & 97\end{array}$

4.2. Flame-sprayed Nickel-Aluminum (Ni-Al) 97

4.2.1. Experimental 97

$\begin{array}{ll}\text { 4.2.2. Results } & 98\end{array}$

4.3. PPS/Clay Nanocomposite 100

4.3.1. Experimental 102

4.3.2. Results 103

$\begin{array}{ll}\text { 4.4. Field Exposure Test } & 106\end{array}$

$\begin{array}{ll}\text { 4.5. Conclusions } & 107\end{array}$

$\begin{array}{lr}\text { 4.6. Publications } & 108\end{array}$

5. Coatings for Condensers 109

5.1. Polyaminopropylsiloxane Coating Derived from Unmodified APST 110

5.1.1. Experimental 110

5.1.2. Results 111

5.2. Ce Oxide/Poly-acetamide-acetoxyl methyl-propylsiloxane

(PAAMPS) Nanocomposite Coating Derived from Ce Acetate$\begin{array}{ll}\text { modified APST } & 114\end{array}$

5.2.1. Experimental 115

$\begin{array}{ll}\text { 5.2.2. Results } & 116\end{array}$

5.3. Field Exposure Tests 118

5.3.1. Experimental 119

$\begin{array}{ll}\text { 5.3.2. Results } & 120\end{array}$

5.4. Conclusions 122

$\begin{array}{ll}\text { 5.5. Patent } & 123\end{array}$

$\begin{array}{ll}\text { 5.6. Publications } & 123\end{array}$

$\begin{array}{ll}\text { 5.7. Conf. Proceeding } & 124\end{array}$ 
6. Coatings for Steam Separators 124

6.1. Experimental 125

6.2. Results 126

$\begin{array}{ll}\text { 6.3. Conclusions } & 131\end{array}$

$\begin{array}{ll}\text { 6.4. Publications } & 131\end{array}$

7. Impact of Work 132

References 133 


\section{Summary}

Corrosion, erosion, oxidation, and fouling by scale deposits impose critical issues in selecting the metal components used at geothermal power plants operating at brine temperatures up to $300^{\circ} \mathrm{C}$. Replacing these components is very costly and time consuming. Currently, components made of titanium alloy and stainless steel commonly are employed for dealing with these problems. However, another major consideration in using these metals is not only that they are considerably more expensive than carbon steel, but also the susceptibility of corrosion-preventing passive oxide layers that develop on their outermost surface sites to reactions with brine-induced scales, such as silicate, silica, and calcite. Such reactions lead to the formation of strong interfacial bonds between the scales and oxide layers, causing the accumulation of multiple layers of scales, and the impairment of the plant component's function and efficacy; furthermore, a substantial amount of time is entailed in removing them. This cleaning operation essential for reusing the components is one of the factors causing the increase in the plant's maintenance costs. If inexpensive carbon steel components could be coated and lined with cost-effective high-hydrothermal temperature stable, anti-corrosion, -oxidation, and -fouling materials, this would improve the power plant's economic factors by engendering a considerable reduction in capital investment, and a decrease in the costs of operations and maintenance through optimized maintenance schedules.

Over the past eight years, the objective of the R\&D work at Brookhaven National Laboratory (BNL) in U.S. Department of Energy (DOE) Geothermal Materials Program was to develop advanced coating material systems with upgraded corrosion-, erosion-, and fouling-prevention performances that extend the lifecycle of carbon steel-based plant components, including heat exchangers, heat exchanger tube/sheet or pipe/pipe joint areas, wellheads, condensers, and steam separators, which encounter very harsh geothermal environments. Since these plant components operate in chemically, physically, and thermally different environments, the material criteria of developing the coating systems depend on the particular components. Success would provide information on new material synthesis, processing technologies, and the specific characteristics of materials, as well as on how to eventually scale up the coating 
technologies necessary to adequately protect carbon steel-based plant's components in corrosive geothermal environments.

\section{Coatings for Heat Exchanger (HX) Tubes}

Economic utilization of the binary working fluids in geothermal energy conversion cycles would dramatically increase the size of the exploitable portion of any hydrothermal resource. The components of the HX, 800 tubes on average, shell, and sheet, represent by far the major cost in a binary plant. Thus, if inexpensive carbon-steel tubes could be coated with a thermally conductive material that resists corrosion, oxidation, and fouling, then there would be a marked reduction in both the capital investment and the maintenance costs of the heat exchanger.

Based upon all the data we obtained from 1998 through 2006, the following were the criteria for the liner systems being developed in this program: 1) Continuous operation at a hydrothermal temperature of $200^{\circ} \mathrm{C}$; 2) thermal conductivity $>1.0$ $\mathrm{kcal} / \mathrm{hr} . \mathrm{m}^{\circ} \mathrm{C}$; 3) cost of $<\$ 1.0 / \mathrm{ft}$; 4) ionic impedance of lining film after 15-day-exposure to $200^{\circ} \mathrm{C}$ brine: $>1 \times 10^{8} \mathrm{ohm}-\mathrm{cm}^{2}$; 5) oxidation rate (O/C atomic ratio) of liners' surfaces after 15 -day-exposure to $200^{\circ} \mathrm{C}$ brine: < 0.05 ; 6) abrasive wear rate by $\mathrm{SiO}_{2}$ grit (particle size of $15 \mu \mathrm{m}$ ) under $150 \mathrm{~m} / \mathrm{s}$ velocity and $0.6 \mathrm{MPa}$ pressure for liners' surfaces after 15-day-exposure to $200^{\circ} \mathrm{C}$ brine: $<0.1 \mu \mathrm{m} / \mathrm{min}$; 7) bond strength of liner to tube: >5.0 MPa; 8) tensile strength of lining film before exposure: > 60.0 MPa; 9) low surface energy of liner: contact angle $>80^{\circ}$ of water droplet on liner surfaces; and, 10) selfrepairing properties. To meet these material criteria, BNL developed smart, highperformance polyphenylenesulfide (PPS)-based composite coating systems consisting of PPS as the matrix, polytetrafluoroethylene (PTFE) as the antioxidant, micro-scale carbon fiber as the thermal conductor and reinforcement, dicalcium aluminate powder as the self-repairing filler, nanoscale boehmite crystal as the wear resistant filler, and crystalline zinc phosphate as the primer. Importantly, the zinc phosphate primer played a very important role in improving the adherence of carbon steel to the coating and in mitigating cathodic corrosion of the underlying steel.

A very promising result was obtained from a two-year-long field validation test of this liner at the Mammoth Pacific power plant, CA, a site operating at a brine temperature 
of $160^{\circ} \mathrm{C}$. The liner adequately protected the 20 -ft.-long carbon steel HX tubes ( $\sim 1.0 \mathrm{in}$. O.D) against corrosion, while minimizing the deposition of irremovable calcium silicate and silica scales. Also, this test result verified that the PPS liner satisfactory withstood the $160^{\circ} \mathrm{C}$ brine and greatly resisted its permeation. In contrast, the surfaces of the unlined stainless steel tubes were very receptive to the deposition of calcium silicate hydrate and silica scales, which strongly adhereed to the tubes.

This encouraging result inspired us to further test its performance at an upgraded brine temperature of $200^{\circ} \mathrm{C}$ in the Puna power plant, HW. Our research team, inlcuding staff from BNL, the National Renewable Energy Laboratory (NREL), and Thermochem, Inc. conducted four-week field validation tests for PPS composite-lined 20-ft.-long tubes with a small diameter of less than $1 / 2$ in. Post-test analyses revealed that this composite liner withstood hot brine at $\sim 200^{\circ} \mathrm{C}$ throughout this abbreviated exposure time. However, we observed some blistering and local delamination of the liners, in particular, near the inlet areas of brine temperatures of $\sim 200^{\circ} \mathrm{C}$ and $\mathrm{pH} 3.5$. The primarily reason for the local delamination and blisters was due to the irregular coverage of the inner surfaces of the small diameter tubes by the crystalline zinc phosphate primer, and was not due to any hydrothermal degradation of the liners. Thus, the standard formulation of the zinc phosphating solution did not offer as effective a protection for small diameter tubes as it did for those with a normal diameter of $\sim 1$ in. Chemical modification of the standard $\mathrm{ZnPh}$ solution was required to deposit a rough crystalline primer layer of at least $10 \mu \mathrm{m}$ thickness for tubes with a diameter of $<0.5$ in.

Nevertheless, Curran International Corp., commercialized this PPS-based lining material system under the trade name "CurraLon". The company estimated that the capital costs of the heat exchanger, containing on average 800 tubes, would be strikingly reduced by $\sim 83 \%$ and $\sim 80 \%$, compared to those of titanium- and stainless steel-based heat exchangers, respectively. Consequently, in 2003, this high-performance PPS composite lining system was selected for the prestigious "Research and Development (R\&D) 100 Award" in 2002 and "Federal Laboratory Consortium for Technology Transfer (FLC) Award”. 


\section{Coatings for HX Tube/Sheet or Pipe/Pipe Joint Areas}

Coatings that are curable at low temperature $<80^{\circ} \mathrm{C}$, yet possess highhydrothermal temperature stability are needed for preventing the corrosion at HX tube/sheet and pipe/pipe joints after roller expansion or welding joining process. None of the coatings tested can withstand exposure to the high compressive strengths and high temperatures of the joining process. Hence, the joint areas must be recoated with materials possessing the following four properties: 1) Hydrothermal stability of $>200^{\circ} \mathrm{C}$; 2) good adherence to the joint areas; 3) ability to cure well at temperatures up to $80^{\circ} \mathrm{C}$; and, 4) ability to be applied with a paint brush or spray gun.

BNL explored the usefulness of a room temperature-curable poly(tetrafluoroethylene)/(hexafluoropropylene) (PTFHFP) polymer coating for mitigating the corrosion of carbon steel joints in $200^{\circ} \mathrm{C}$ brine. After 20 days exposure, the superficial layer of this coating suffered hydrothermal oxidation, thereby diminishing its maximum effectiveness in preventing the corrosion of underlying steel. Our focus next centered on evaluating the NASA-developed polybezimidazole (PBI) polymer coating that possesses outstanding thermal stability (a high heat-deflection temperature of $450^{\circ} \mathrm{C}$ ). Unfortunately, when this room-temperature curable PBI film was exposed to $200^{\circ} \mathrm{C}$ brine, it underwent hydrolysis. Consequently, the coating was hydrothermally degraded, and had delaminated from the underlying steel joints after a 14-day exposure.

Solvay Advanced Polymers, L.L.C. provided us with a newly developed solventdissolvable polyarylethersulfone (PES) polymer. Its specific characteristics were its high glass-transition and softening temperatures of $260^{\circ}$ and $350^{\circ} \mathrm{C}$, respectively. Although the autoclave validation test was carried out only for two weeks, the PES coating gave some encouraging results; namely, it adequately protected the jointed steel against corrosion in $200^{\circ} \mathrm{C}$ brine. However, at $\geq 250^{\circ} \mathrm{C}$, the PES underwent severe hydrothermal oxidation, causing cleavage of its sulfone- and ether-linkages, and opening of phenyl rings. Consequently, the disintegration of the PES structure by hydrothermal oxidation was detrimental to the maximum efficacy of the coating in protecting the steel against corrosion, allowing the corrosive electrolytes to infiltrate easily through it.

Thus far, a hydrothermally self-advancing hybrid material, prepared by blending two starting materials, water-borne styrene acrylic latex (SAL) as the matrix and calcium 
aluminate cement (CAC) as the hydraulic filler, has been identified as the most promising room-temperature curable anti-corrosion coating for these joints exposed to $250^{\circ} \mathrm{C}$ brine. The following two major factors supported the self-improving mechanisms of the coating during autoclaving: First was the formation of a high temperature stable polymer structure of Ca-complexed carboxylate groups containing SAL (Ca-CCG-SAL) resulting from hydrothermal reactions between SAL and CAC; second was the growth with continuing exposure time of crystalline calcite and boehmite phases coexisting with CaCCG-SAL. These two factors promoted the conversion of the porous microstructure in the non-autoclaved coating into a densified one after 7 days exposure. The densified microstructure not only considerably reduced the conductivity of corrosive ionic electrolytes through the coatings' layers, but also contributed to the excellent adherence of the coating to the underlying steel' s surface.

\section{Coatings for Wellhead Components}

Wellhead components, including a flow line and tee, valve, and casing head, encounter a very harsh environment with a flow velocity of $\sim 3 \mathrm{~m} / \mathrm{sec}$ of brine at $250^{\circ} \mathrm{C}$. If their components were made of inexpensive carbon steel instead of titanium alloybased metals, $250^{\circ} \mathrm{C}$ hydrothermal stable coatings would be needed to protect the component's surface against corrosion. Three different coatings were evaluated: The first was a titanium carbonitride $[(\mathrm{Ti}(\mathrm{CN})]$ coatings generated by chemical vapor deposition (CVD) technology; the second was a nickel-aluminum (Ni-Al) coating produced by flame-spray technology; and the third was a PPS/Clay coating made using nanocomposite technology.

Both the CVD-Ti(CN) and flame-sprayed Ni-Al coatings failed when exposed in $250^{\circ} \mathrm{C}$ brine environment. Two undesirable attributes caused their failure as corrosionpreventing coatings: One was the susceptibility of these coatings to hydrothermal oxidation reactions with hot brine; the other was the inherent open structure of these coatings that allowed the hot brine to permeate them easily, causing the development of corrosion-induced stress cracks in the underlying carbon steel. The degradation of $\mathrm{Ti}(\mathrm{CN})$ in such an environment took place through the processes of oxidation 6 chlorination 6 dissolution. The hydrothermal oxidation of Ni-Al coating led to the formation of $\mathrm{Al}_{2} \mathrm{O}_{3}$ as 
the major scale compound and $\mathrm{NiO}$ as the minor one, and their subsequent accumulation and spallation on the carbon steel.

Since the melting temperature of PPS was around $250^{\circ} \mathrm{C}$, it was reasonable to assume that this material can withstand the brine temperatures up to $200^{\circ} \mathrm{C}$. Undoubtedly, increasing the melting temperature of PPS is an inevitable next step, if its potential application is targeted towards protecting carbon steel components at $\geq 250^{\circ} \mathrm{C}$ against corrosion and scale deposition. To enhance the melting point of PPS, the polymer/clay nanocomposite technology was adapted by using montomorillonite (MMT) clay as the alternative nanoscale filler. This nanofiller conferred three advanced properties on the semi-crystalline PPS: First, it raised its melting point by nearly $40^{\circ} \mathrm{C}$ to $290^{\circ} \mathrm{C}$; second, it increased its crystallization energy, implying an excellent adherence of the nanofillers' surfaces to PPS in terms of a good interfacial bond; and, third, it abated the degree of its hydrothermal oxidation due to sulfide $\rightarrow$ sulfite linkage transformations. When this advanced PPS nanocomposite was used as a corrosion-preventing coating for carbon steel in a simulated geothermal environment at $300^{\circ} \mathrm{C}$, a coating of $\sim 150 \mu \mathrm{m}$ thickness adequately protected the steel against hot brine-caused corrosion.

\section{Coatings for Condensers}

With the increased consumer demand for electricity from geothermal binary plants during the most economically valuable summer season, one very important issue is the impairment of the efficiency of the air-cooled condensers, thereby causing $30 \%$ reduction in the plant's net monthly energy delivery compared with that in the winter. A simple method to deal with this problem is to directly spray inexpensive relatively clean cooled geothermal brine over the surfaces of the aluminum-finned steel tubing condenser. Although this method is very attractive, a concern raised about spraying the brine is the likelihood of corroding the condenser's components, aluminum fins and carbon steel tubes, as well as depositing geothermal brine-induced mineral scales on them. To deal with this problem, the anti-corrosion and anti-fouling coatings are needed.

The aluminum fins circling the surfaces of steel tube are of many different shapes and structures; some of which they have zigzag- and wave-shaped conformations. Further, the distance between the fins is less than $3 \mathrm{~mm}$, while the height of fin attached 
to the steel tube is $\sim 15 \mathrm{~mm}$. Based upon their unique features and the information obtained from field and in-house tests over the past three years, the following were the design criteria for the coatings developed in this program: 1) Low surface tension of $<60$ dynes/cm, allowing them to easily permeate through and wick between the fins; 2) excellent wetting behavior on the surfaces of both aluminum and steel: a contact angle of $<70^{\circ}$ of a precursor solution droplet on both metal surfaces; 3) a hydrophobic surface to confer water-repellent and -shedding properties: low surface energy of $<30 \mathrm{~mJ} / \mathrm{m}$; 4) retention of thermal conductivity by a thin coating film of $<100 \mu \mathrm{m}$, so to reduce material cost; 5) coating cost of $\left.<\$ 0.015 / \mathrm{ft}^{2} ; 6\right)$ ASTM salt-spray resistance of $>1000$ hours; 7) good adherence to aluminum and steel: required strength of interfacial bond was unknown; 8) durability: withstand 10,000 brine wet/dry cycles; 9) chemical inertness of their surfaces to brine minerals to prevent scale deposition; and, 10) low conductivity of corrosive ions: ionic impedance of $>1 \times 10^{5} \mathrm{ohm}-\mathrm{cm}^{2}$.

To design the coatings meeting for this material criteria, BNL succeeded in developing a new technology of self-assembly nanosynthesis that allowed the assembly of a the nanocomposite structure consisting of the nanoscale rare-earth metal oxides as the corrosion inhibitors, and water-based organometallic polymer (OMP) as the hydrophobic matrix. Among the rare-earth metal oxides, the environmentally benign cerium (Ce) oxide was employed in this nanocomposite system. Using this synthesis technology involving three spontaneous reactions, viz., condensation, amidation, and acetoxylation, between the Ce acetate dopant and aminopropylsilane triol (APST) as the film-forming precursor aqueous solution, a synthetic OMP material was composed of Ce oxide as the nanoscale filler and poly-acetamide-acetoxyl methyl-propylsiloxane (PAAMPA) polymer in a family of OMP. This nanocomposite coating extended the useful lifetime of steel exposed in a salt-fog chamber at $35^{\circ} \mathrm{C}$ from only $\sim 10$ hours to 768 hours. Furthermore, this coating system far better protected an aluminum substrate from the corrosion that it did one of steel. The salt-spray resistance of film-covered aluminum panels was strikingly extended to more than 1440 hours compared with $\sim 40$ hours for bare aluminum.

Under an extremely harsh environment, exemplified by the 24,500 brine wet/dry cycle field fatigue test conducted by NREL at the Mammoth power plant, the Ce 
oxide/PAAMPS nanocomposite coatings displayed very promising results in protecting two metal components, the aluminum fins and carbon steel, of air-cooled condensers against corrosion, and in minimizing the deposition of scales. However, two critical issues to further improve corrosion-preventing performance of this coating remained unanswered: One is the poor coverage of the coating over the sharp edges of the ringshaped fin; the other issue is to explore more effective nanoscale rare earth metal oxides in inhibiting the cathodic corrosion reaction of metal surfaces underneath the coating, instead of using Ce oxide.

Nevertheless, BNL filed a patent entitled "Self-assembly Nanocomposite Coatings” through BNL's technology transfer office.

\section{Coatings for Steam Separators}

The steam separator plays a pivotal role in minimizing the extent of erosion of the turbine blades brought about by brine-laden steam containing hard mineral particles impinging on the blade's surfaces. Thus, the metal components of separators must possess excellent resistance to corrosion and abrasive wear at hydrothermal temperatures up to $210^{\circ} \mathrm{C}$. Currently, despite being very expensive, Inconnel $625 \mathrm{Ni}-\mathrm{Cr}$ alloy is extensively used in separators to lessen such erosion. The focus of our work in collaboration with Two-Phase Engineering \& Research, Inc. centered on developing and evaluating candidate materials as the internal coatings for the carbon steel separators.

Like Inconnel 625, the coatings must not only offer good corrosion- and scalepreventing performance at brine temperatures up to $210^{\circ} \mathrm{C}$, but also their surfaces must have three important properties: 1) Low friction; 2) low surface free energy corresponding to high water repellency; and, 3) resistance to abrasive wear.

We evaluated usefulness of a Teflon-rich PPS coating system as slip- and water repellent-enhancing, anti-corrosion barrier film. We demonstrated that it afforded outstanding slipperiness and water repellent properties because of its low surface free energy. In addition, although the Teflon-rich coating was immersed for 35 days in $\mathrm{CO}_{2}$ laden brine at $300^{\circ} \mathrm{C}$, it significantly contributed to preventing the permeation of moisture and corrosive electrolytes through the film, so mitigating the corrosion of carbon steel. We also explored, in detail, the changes in the kinetic coefficient of friction (KCOF) of 
the Teflon-rich PPS coatings' surfaces as a function of exposure time in $\mathrm{CO}_{2}$-laden brine at $300^{\circ} \mathrm{C}$. A very low KCOF of 0.19 was recorded from the Teflon-rich PPS surfaces before exposure to hot brine, suggesting that this coating was very slippery; this value represented a decrease of $47 \%$ and 2.6-fold compared with that of the PPS surface without Teflon and the stainless steel (SS) surface, respectively. After exposing the Teflon-rich PPS to hot brine, the KCOF value rose by $21 \%$ to 0.23 in the first 7 days exposure; beyond then, it seemed to level off. In contrast, the friction of the SS surfaces increased from 0.5 before exposure to 0.9 after 14 days exposure, raising concerns that the enhanced surface roughness accompanying this increment in friction might promote the deposition of scales.

\section{Collaborators}

The collaborators given below supported this project and expedited its progress.

Dr. Keith Gawlik (Collaborator, National Renewable Energy Laboratory)

Dr. Paul Hirtz (Collaborator, Thermochem, Inc.)

Mr. Robert Sullivan (Co-sharing partner, Mammoth Project) Contribution: Field validation tests

Mr. Brian Koenig (Co-sharing partner, CalEnergy Operating Corp.) Contribution: Field validation tests

Mr. Mike Kaleikini (Co-sharing partner, Puna Geothermal Venture)

Contribution: Field validation tests

Mr. Douglas Jung (Co-sharing partner, Two-Phase Engineering \& Research)

Contribution: Assessment of technical and economical feasibility of BNL-developed coating systems 


\section{Introduction}

One way to reduce capital investment and the expenses of operating and maintaining geothermal power plants with brine temperatures up to $300^{\circ} \mathrm{C}$ is to use inexpensive carbon steel components coated with cost-effective anticorrosion and antifouling materials instead of the very expensive stainless steel, titanium alloy, and inconel components that commonly are employed as a low corrosive metals. In this concept, the coatings play a pivotal role in extending the lifecycle of the carbons steel components including heat exchangers, wellheads, condensers, and steam separators, eventually lowering the costs of electricity generated from these plants.

To achieve this goal, the R\&D work at the BNL in the DOE geothermal materials program was focused on developing advanced coating material systems with upgraded corrosion-, erosion-, hydrothermal oxidation-, and fouling-prevention performance for these plant components in very harsh geothermal environments. The technical approach encompassed innovative syntheses and processing, and short-term in-house and field exposure tests for evaluating the reliability of the new types of coating materials. The work also was designed to obtain a fundamental understanding of the characteristics of these coating systems before applying them to full-scale metal substrates. Once a fundamental formulation of coating materials was established, our focus next was shifted to developing the coating and lining process technologies that confer the maximum performance of the formulated material systems, and can be scaled up. The coated and lined full-scale metal components then were deployed in geothermal plants to conduct the field validation tests and to identify the potential coating systems suitable for mitigating corrosion and deposition of scales on metal components.

Below are the aspects for reducing capital investment and maintenance costs of these plant components.

\section{Heat Exchangers (HXs)}

The economic utilization of binary working fluids in geothermal energy conversion cycles would dramatically increase the size of the exploitable portion of any hydrothermal resource. The components of the HX, such as tubes, shell, and sheet, represent by far the major cost in a binary plant. The stainless steel and titanium alloy $\mathrm{HX}$ 
tubes presently used in such binary-cycle plants afford great protection against corrosion caused by hot brine. However, there is one serious concern about using these expensive, high-grade metal alloy tubes. The concern is the inevitable formation of the corrosionpreventing passive oxide layers on their outermost surface sites. The oxide layers are detrimental in that the tubes' surfaces become more susceptible to the reactions with silicate and silica scales, developing a strong adherence to them. The accumulation of such multiple layers of scales promoted by the interfacial bonds between the scale and oxide layer not only impairs the HX's function and efficacy, but also entails a substantial amount of time to remove them, so that this cleaning operation essential for reusing the tubes is very costly. Thus, if inexpensive carbon-steel tubes could be coated with a thermally conductive material that resists corrosion, oxidation, and fouling, then there would be a marked reduction in both the capital investment and the maintenance costs of the heat exchanger, which contains 800 tubes on average.

\section{$\underline{\text { HX Tube/Sheet and Pipe/Pipe Joint Areas }}$}

The completion of some plant components, such as the HX unit and long pipeline, requires joints. Roller-expansion and welding processes are among the most popular ways to make the HX tube-to-tube sheet joints, and also the pipe-to-pipe joints. In the former process, the thickness of the walls of the carbon steel HX tubes are reduced by $\sim 6$ $\%$ as they are rolled into a tube sheet. Currently, none of available coatings can withstand such high compressive strength and abrasive wear during this expansion. Likewise, welding at high temperature causes thermal decomposition of the coatings. Hence, the joint areas, of $\sim 10.0 \mathrm{~cm}$ wide, must be recoated in the factories or in the field with materials having the following four important properties: 1) good curability at low temperatures up to $80^{\circ} \mathrm{C}$; 2) hydrothermal stability of $>200^{\circ} \mathrm{C}$; 3) good adherence to the

joint areas; and, 4) be suitable for application with a paintbrush or spray gun. In addition, the material cost must be as low as possible.

\section{Wellhead Components}

One specific area of application in the geothermal plants is the production wellhead, consisting of a flow line and tee, valve, and casing head, that encounter a very 
harsh environment with a flow velocity of $\sim 3 \mathrm{~m} / \mathrm{sec}$ of brine at $250^{\circ} \mathrm{C}$. At present, a titanium alloy-based metal is commonly used in assembling the wellhead. Hence, if this could be replaced by an inexpensive carbon steel wellhead coated with a cost-effective, anti-corrosion and -fouling material, which withstands $300^{\circ} \mathrm{C}$ hydrothermal temperature, capital expenditure would fall considerably.

\section{Condensers}

With the increased demand for selling electricity in geothermal binary plants during the most valuable summer season, one very important issue is the impairment of the efficiency of the air-cooled condensers, thereby causing $30 \%$ reduction in the plant's net monthly energy delivery compared with that in the winter [1]. A simple method to deal with this problem is to directly spray inexpensive relatively clean cooled geothermal brine over the surfaces of the aluminum-finned steel tubing condenser. As expected, such sprayed condensers then attain outputted the same generating capacity for electricity as under wintertime conditions. Although this method is very attractive, a concern raised about spraying the brine is the likelihood of corroding the condenser's components, aluminum fins and carbon steel tubes, as well as depositing geothermal brine-induced mineral scales on them. There is no doubt that such negative phenomena, corrosion and scale deposition, cause the impairment of the condenser's maximum function and efficacy. To deal with this problem, the anti-corrosion and anti-fouling coatings are needed to ensure that the condensers have a long useful lifetime free from any corrosion and hard-to-remove scale deposits.

\section{$\underline{\text { Steam Separators }}$}

In geothermal power plants, a high temperature steam mixed with brine containing minerals such as silicate and silica, is extracted under pressure as a resource of renewable energy from wells, 300 to 3,000 m deep. The extracted steam then flows into the steam separator for purification. The steam separator plays a pivotal role in minimizing the extent of erosion of the turbine blades brought about by the brine-laden steam containing hard mineral particles impinging on the blade's surfaces. Thus, the metal components of separators must possess excellent resistance to corrosion and 
fouling at hydrothermal temperatures up to $210^{\circ} \mathrm{C}$ because once the topographical features of the internal surfaces of separators are altered by a build up of scale, pitting corrosion, and enhanced asperity due to abrasive wear, their maximum efficacy in improving the quality of steam diminishes. Currently, despite it's being very expensive, Inconnel $625 \mathrm{Ni}-\mathrm{Cr}$ alloy is extensively used to deal with such an alteration. If Inconnel 625 can be replaced by an inexpensive carbon steel coated with cost-effective, high temperature-stable, anti-corrosion and anti-fouling materials, there is no doubt that the cost of separators would be considerably reduced. Hence, emphasis in this study was directed towards developing and evaluating candidate materials as the internal coatings for carbon steel separators. Like Inconnel 625, the coating to be developed must not only offer good corrosion- and scale-preventing performance at brine temperatures up to $210^{\circ} \mathrm{C}$, but also its surfaces must offer the following three important properties; 1 ) slipperiness, 2) water repellency, and 3) resistance to abrasive wear.

\section{Coatings for HX Tubes}

The melt-crystallized polyaryl thermoplastic engineering polymers, such as polyphenylenesulfide (PPS), polyphenyletheretherketone (PEEK), and polyphenyletherketone (PEK), have come chemical features consisting of aromatic backbone chains coupled with oxygen, ketone, and/or sulfide. When these liner polymers are left in an oven at a temperature above their melting point of $>250^{\circ} \mathrm{C}$, chain extension of the main phenyl groups caused by melting leads to molecular orientation, which is reflected in the crystallization of the polymers during cooling from the melting temperature to a lower temperature [2-4]. Such crystallization behavior of the polyaryls gives them specific desirable characteristics, such as high temperature stability, high

radiation, chemical, and hydrothermal resistance, and good mechanical and dielectric properties. Thus, they have become of increasing interest for applications in coatings, as adhesives, and in composites.

Among them, PPS was chosen as the high-temperature performance lining material for carbon steel $\mathrm{HX}$ tubes operating at brine temperatures up to $200^{\circ} \mathrm{C}$ because of its lower price and its larger production compared with those of other polyaryls [5]. However, this lining material was required to improve the following seven properties: (1) 
adherence to underlying steel; (2) thermal conductivity in order to increase the thermal transfer efficiency of liner; (3) tensile strength and elongation; (4) corrosion-preventing performance; (5) insusceptibility to hydrothermal oxidation to avoid the irremovable scale deposits; (6) resistance to abrasive wear; and, (7) self-repairing mechanisms to heal the cracks generated on the liner's surface during its use in the power plants.

\subsection{Adherence to Underlying Steel}

An ideal coating that will protect steel from corrosion is one that acts as a barrier against the attack of corrosive reactants, such as oxygen, water, electrolyte species (eg., $\mathrm{H}^{+}, \mathrm{Na}^{+}, \mathrm{NH}_{4}{ }^{+}, \mathrm{Cl}^{-}, \mathrm{SO}_{4}{ }^{2-}, \mathrm{NO}_{2}{ }^{-}$), and gases (eg., $\mathrm{O}_{3}, \mathrm{SO}_{2}, \mathrm{NO}_{\mathrm{x}}$ ), in natural or artificial environments. Unfortunately, all polymeric coatings are permeable to these reactants in some different degrees. Thus, an important consideration is that when the reactants reach the steel interfaces beneath the coating layers, corrosion occurs at the interface between coating and steel. Once corrosion is initiated, the growth of corrosion products at the interfaces promotes wedging and blistering, which put tremendous stress on the interfacial side of coating film; consequently, there is localized delamination and buckling of the stressed coating layers [6]. In addition, if the coating films become blemished, the underlying steels react with the reactants that have penetrated through the defect. Such a reaction is commonly called the two-step corrosion reaction; a cathodic reaction, $\mathrm{H}_{2} \mathrm{O}+1 / 2 \mathrm{O}_{2}+2 \mathrm{e}^{-} \rightarrow 2 \mathrm{OH}^{-}$, which occurs under the coating adjacent to the defect, and an anodic reaction, $\mathrm{Fe}+2 \mathrm{e}^{-} \rightarrow \mathrm{Fe}^{2+}$, which occurs at a defect in the coating $[7,8]$. Considerable attention in this corrosion process has been given to the high $\mathrm{pH}$ environment created at the cathodic sites; namely, the alkali generated beneath the coating not only causes the formation of corrosion products, but also promotes the alkalicaused degradation of polymeric coatings [9-11]. Subsequently, these phenomena occurring at the interfaces between the coatings and the steels lead to the cathodic delamination of the coatings from the steels. Thus, it is very important to tailor the surfaces of carbon steel prior to application of PPS coating. The surface tailoring not only acts to inhibit the onset of the cathodic reaction, and also improves the adherence of coating to underlying steel's surface. 
For such tailoring technologies, zinc or zinc alloy and zinc phosphate ( $\mathrm{ZnPh})$ conversion primers are often introduced into the intermediate layers as a post-treatment of the steel surfaces [12]. Among them, the $\mathrm{ZnPh}$ primer was employed in this work because the open surface structure (Figure 1) of the interlocked insoluble $\mathrm{ZnPh}$ crystal primer layers contributed significantly to the formation of a strong mechanical interlocking reaction with the polymeric topcoat systems, thereby enhancing the magnitude of the adhesive force at the primer/topcoat interfaces [13].

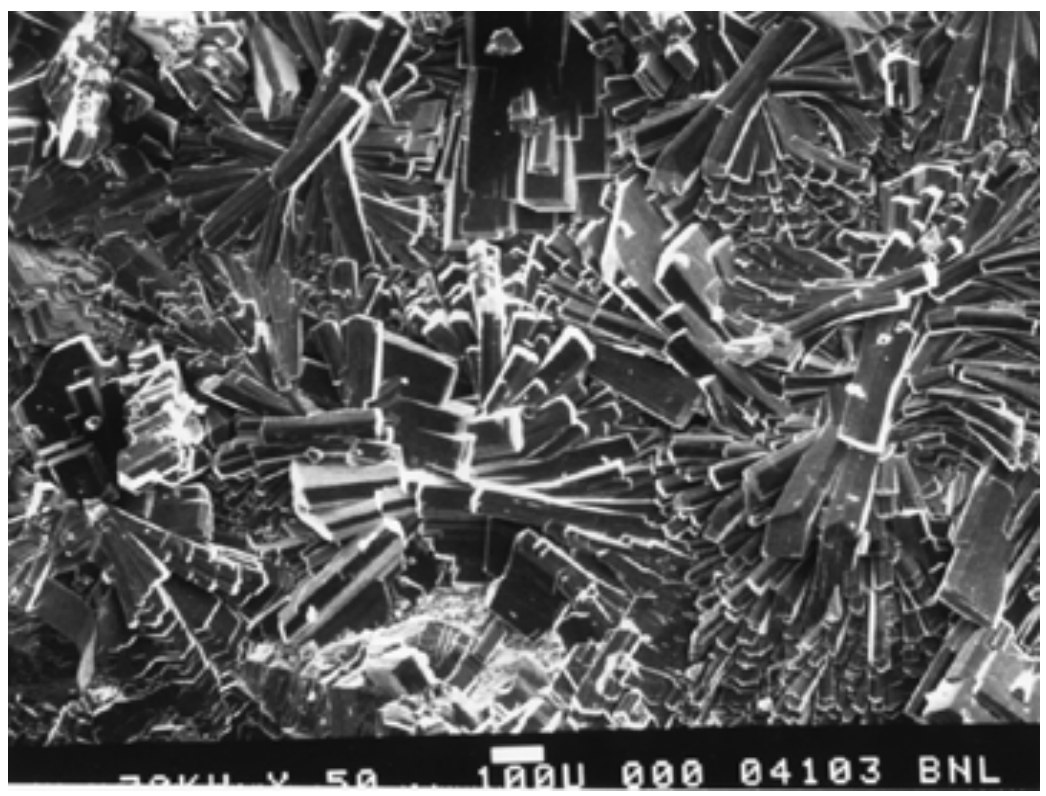

Figure 1. Microphotograph of zinc phosphate conversion primer.

On the basis of this information, emphasis was directed towards exploring the changes in chemistry and morphology occurring at the interfaces between PPS coating and phosphated steels after heating-cooling fatigue tests in an accelerated corrosive environment at 25 to $200^{\circ} \mathrm{C}$. All the information obtained was correlated directly with the results from cathodic delamination tests for PPS coatings.

\subsubsection{Experimental}


The metallic substrate used was commercial ALSI 1008 carbon steel. A thermoplastic PPS powder with a particle size of $<60 \mu \mathrm{m}$ was obtained from Ticona Co. It has a high melt flow at its melting point around $240^{\circ} \mathrm{C}$. The $45 \mathrm{wt} \%$ PPS powder was mixed with $55 \mathrm{wt} \%$ isopropyl alcohol to make the slurry precursor. Before depositing the PPS slurry on the metal, the surfaces of the metals were covered with a $\mathrm{ZnPh}$ primer by immersing them for 10 min into a zinc phosphate solution consisting of a $3.8 \mathrm{wt} \%$ zinc orthophosphate, $7.5 \mathrm{wt} \%$ phosphoric acid, $1.0 \mathrm{wt} \%$ calcium nitrate, $2.0 \mathrm{wt} \%$ zinc nitrate, $2.4 \mathrm{wt} \%$ ferrous sulfate, and $83.3 \mathrm{wt} \%$ tap water at $80^{\circ} \mathrm{C}$. The PPS coating was deposited on the primed steel panels' surfaces in the following way: First, the primed panels were dipped into the slurry, and withdrawn slowly; second, the slurry-covered panels were left in an atmospheric environment at $25^{\circ} \mathrm{C}$ for 20 hours to volatilize the alcohol, and concomitantly, to promote the conversion of the slurry into a sintering layer; and finally, the panels were heated in air at $300^{\circ} \mathrm{C}$ for 3 hours until the sintered layers reached their optimum melt-flow, and subsequently cooled to room temperature to make solid PPS film. The thickness of the coating films ranged from 0.0075 to $0.1 \mathrm{~mm}$.

In all the tasks on the coatings for HXs, the same metallic substrate and PPS powder were used, and also the $\mathrm{ZnPh}$ priming and PPS depositing process technologies described above were employed to prepare the coating specimens.

The cyclic fatigue tests for the PPS/ZnPh primer/steel systems were accomplished as follows; the PPS covered steel specimens were directly soaked in a corrosive solution consisting of $1.0 \mathrm{wt} \% \mathrm{H}_{2} \mathrm{SO}_{4}, 3.0 \mathrm{wt} \% \mathrm{NaCl}$, and $96.0 \mathrm{wt} \%$ water, and then the heatingcooling cycle (one cycle $=12$ hours at $200^{\circ} \mathrm{C}+12$ hours at $25^{\circ} \mathrm{C}$ ) was repeated up to 90 times.

\subsubsection{Results}

\subsubsection{Bond Mechanisms at Interfaces Between PPS and ZnPh}

A very encouraging result was obtained from cyclic tests of the PPS-coated panels; namely, there was no sign of delamination and decomposition of PPS coating films after 90 cycles. This fact proved that the bond durability at the interfaces between PPS and $\mathrm{ZnPh}$ is outstanding in such a harsh environment. 
To investigate the structure and chemistry of the interfacial bonds that confer such excellent durability, the cross-sectional areas and both the interfacial PPS and panel sides were explored by scanning electron microscopy (SEM) coupled with energy-dispersive $\mathrm{X}$-ray spectrometry (EDX) and X-ray photoelectron spectroscopy (XPS). Figure 2 shows the SEM images and EDX spectra for the cross-sectional areas of 30 (top) and 90 (bottom) cycled PPS/ZnPh/steel joints. These SEM images clearly demonstrated that the cross-section regions have three different layers. The EDX spectrum (not shown) of top layer in the SEM micrographs indicated that only two elements, $C$ and S, were present, reflecting the PPS coating. No traces of $\mathrm{Cl}$ and $\mathrm{Na}$ were detected in the PPS layers. From the EDX spectrum (not shown) containing a single element of Fe, the bottom layer in the micrograph corresponds to the underlying steel. The SEM image also highlighted that melted PPS penetrates into the open-surface microstructure and micro-size fissures of $\mathrm{ZnPh}$ layer. Such filling of the spaces in the $\mathrm{ZnPh}$ primer layers by PPS appears to increase the extent of mechanical bonding between PPS and primer layers. Attention was focused on the EDX spectra at the location " 1 " in the primer layer, and "2" in the critical boundary region between the primer and steel. Although $\mathrm{SO}_{2}$ gases are emitted from oxidized PPS at high temperatures [14], a surprising fact here was that the S species from PPS was present throughout the primer layers. The detection of $S$ in the primer/steel interfacial areas suggested that $\mathrm{S}$ invaded deeply into the primer layers causing the formation of S-related Fe compounds by reacting with the underlying steel. Considerable attention next was paid to the striking change in the image of the primer layers for the 90cycle panels. A noteworthy difference of from that of the 30-cycles was the development of numerous small cracks. Such a damaged and porous state of the primer layer was implicated by the increased intensity of S element in the EDX spectrum at a location "3", suggesting that the primer layers react with the $\mathrm{S}$ species from PPS.

To further substantiate the results from this cross-sectional examination, the chemical composition and development of the microstructure of the failure surfaces on the panel side removed physically from the PPS coating were studied by SEM-EDX. The results from 30-cycled PPS/primer/panel system is given in Figure 3. No complete 

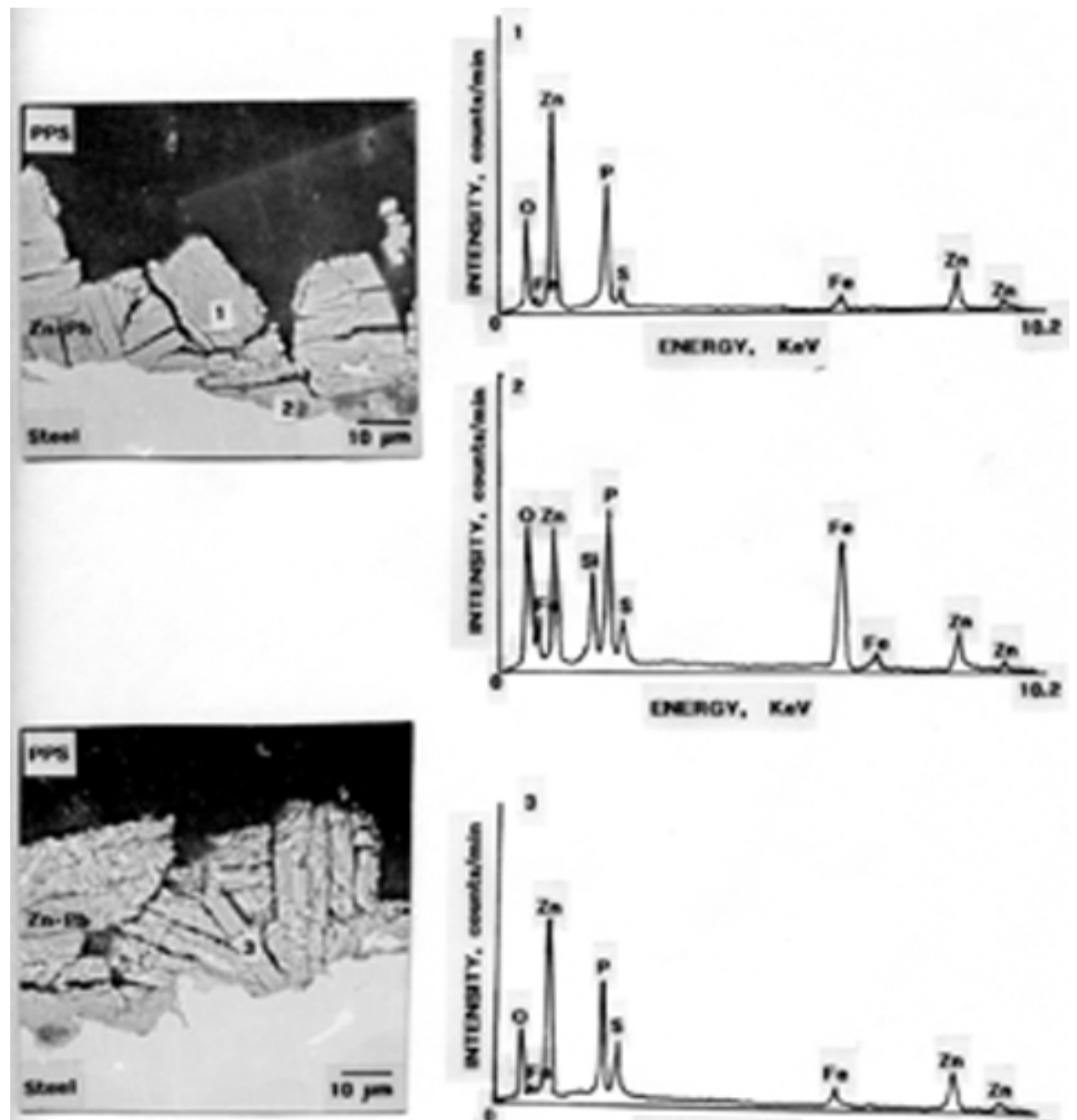

Figure 2. SEM-EDX examinations for the cross-sectional regions of the 30 -cycled (top) and 90-cycled (bottom) PPS/ZnPh/steel joints. 

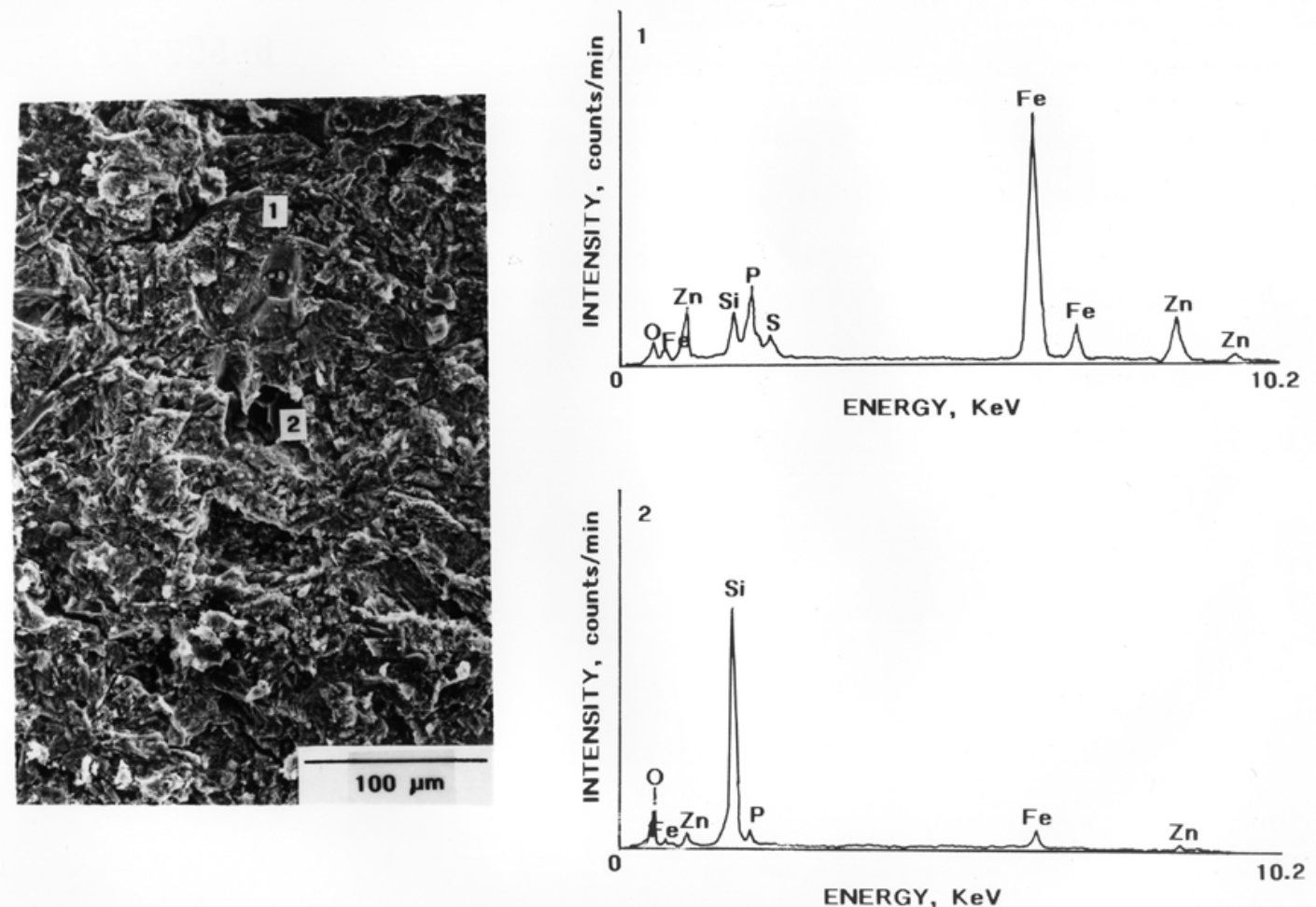

Figure 3. SEM-EDX data of the substrate interface side removed from the PPS coating for the 30 -cycled panels.

covering of $\mathrm{ZnPh}$ primer remained on the panel surfaces; therefore, most of primer layers must have been transferred to the PPS side when the loss of adhesion occurred. The EDX location “1” had highly intense Fe signal, moderately intense signals from Zn and P, while $\mathrm{O}, \mathrm{Si}$, and $\mathrm{S}$ were minor components. The detection of $\mathrm{Zn}$ and $\mathrm{P}$ suggests that some remnants of primer remained on the failed panel side. Location " 2 " showed a different morphology from that of location "1": the EDX pattern on this structure with its sharp edge showed two intense signals of Si and O, revealing the formation of Si oxides as the contaminants of the steel surfaces. As a result, the loss of adhesion takes place in a boundary region between the primer and steel, underscoring that $\mathrm{ZnPh}$ primer adheres more strongly to the PPS coatings than that to steel. 
Next, focus centered on identifying the reaction products at the interfaces between the PPS and $\mathrm{ZnPh}$ primer. This information was obtained by inspecting the $\mathrm{XPS} \mathrm{Zn}_{2 \mathrm{p} 3 / 2}$ region on the failure surface of primer side. Since the interaction of PPS with the $\mathrm{ZnPh}$ may generate the S-related Zn compounds as the reaction products, the two compounds, zinc sulfide $(\mathrm{ZnS})$ and zinc sulfate $\left(\mathrm{ZnSO}_{4} \cdot \mathrm{H}_{2} \mathrm{O}\right)$, were used as reference samples to identify the XPS bonding energy (BE) of the precise peak position in the $\mathrm{Zn}_{2 \mathrm{p} 3 / 2}$ region. The peak positions for these reference samples were $1022.7 \mathrm{eV}$ for $\mathrm{ZnS}$ and $1024.0 \mathrm{eV}$ for $\mathrm{ZnSO}_{4} \cdot \mathrm{H}_{2} \mathrm{O}$. Figure 4 shows the changes in spectral features in the $\mathrm{Zn}_{2 \mathrm{p} 3 / 2}$ region as a function of cycle number. The spectrum of the control (a) reveals only a single symmetric peak at $1024.6 \mathrm{eV}$, attributed to $\mathrm{Zn}$ originating from the $\mathrm{ZnPh}$. A noticeable change in the shape of the $\mathrm{Zn}_{2 \mathrm{p} 3 / 2}$ signal from the 30-cycled panel (b) was the emergence of a new signal at $1022.7 \mathrm{eV}$. According to the reference peaks, this new peak conceivably could be the $\mathrm{Zn}$ in the $\mathrm{ZnS}$ reaction product derived from the interaction between the PPS and $\mathrm{ZnPh}$; the intensity of this peak grows with more cycles. After 90 cycles (d), the peak at 1022.7 eV becomes a dominant component, while the ZnPh-related peak at $1024.6 \mathrm{eV}$ considerably decays. Relating this finding to the SEM exploration early, the development of numerous microfissures in the $\mathrm{ZnPh}$ layer was due to the degradation of $\mathrm{ZnPh}$ layers caused by the in-situ conversion of $\mathrm{ZnPh}$ into the $\mathrm{ZnS}$ as reaction product.

\subsubsection{Cathodic Delamiantion}

An important question was the ability of $\mathrm{ZnS}$ to reduce the rate of cathodic delamination of the coating films from the $\mathrm{ZnPh}$ primer layers. Because the $\mathrm{ZnS}$ compound is substantially insoluble in the water as well as alkaline solution, it is possible to assume that this reaction product would suppress the delamination of polymer film caused by alkali-catalyzed dissolution of $\mathrm{ZnPh}$ layers occurring at the cathodic reaction side, $\mathrm{H}_{2} \mathrm{O}+1 / 2 \mathrm{O}_{2}+2 \mathrm{e}^{-} \rightarrow 2 \mathrm{OH}^{-}$. The cathodic delamination tests for the PPS-coated $\mathrm{ZnPh}$ panels after cycling were conducted in an air-covered $1.0 \mathrm{M} \mathrm{NaCl}$ solution using an 


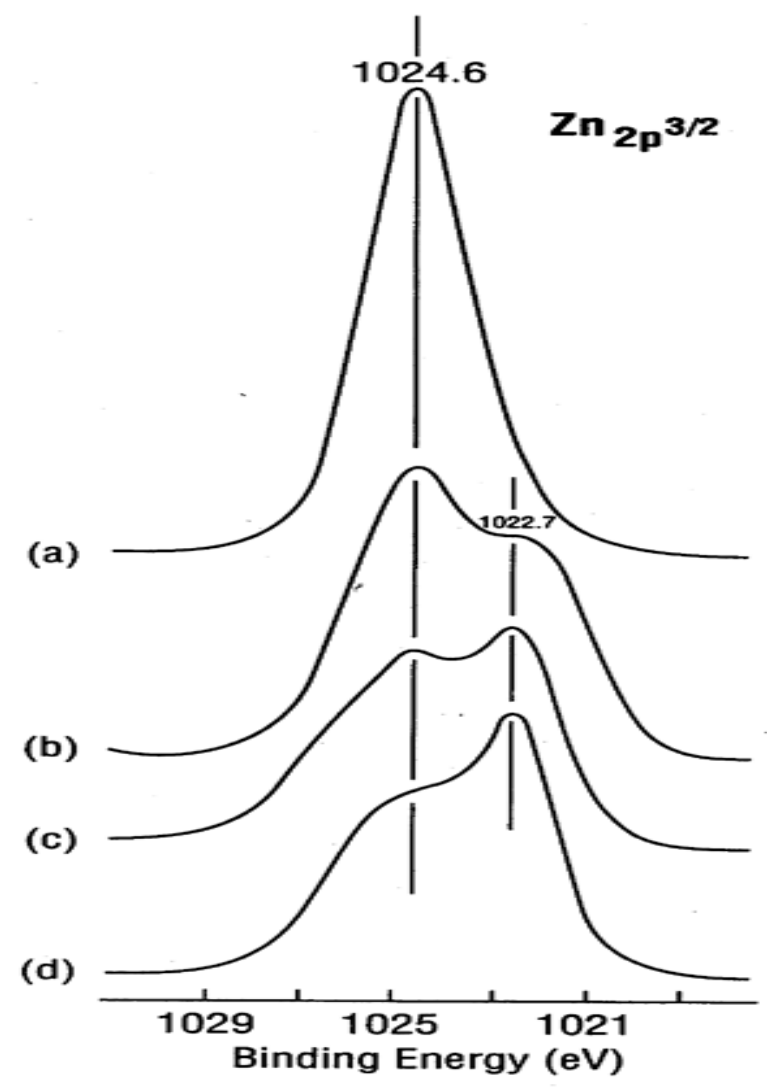

Figure 4. XPS $\mathrm{Zn}_{2 \mathrm{p} 3 / 2}$ region for the interfacial steel side of (a) 0-, (b) 30-, (c) 60-, and (d) 90-cycled PPS/ZnPh/steel joints.

applied potential of $-1.5 \mathrm{~V}$ vs SCE for 8 days (Figure 5). A defect was made using a 1 mm diameter drill-bit. After exposure, the panels were removed from the cell and allowed to dry. The coating was removed by cutting, revealing a light gray, delaminated region adjacent to the defect. The delaminated areas of PPS films from the primer were measured for 0-, 20-, 30-, 60-, and 90-cycled panels after eight days of cathodic delamination tests (Figure 6). As is seen, the rate of delamination was progressively reduced as a function of cyclic number. The delamination value of $\sim 1.8 \mathrm{~mm}^{2}$ for the 90 cycled panels was $\sim 20$ times lower than that for the control panels. Such a dramatic reduction was due mainly to the rate of in-situ conversion of the $\mathrm{ZnPh}$ layers into $\mathrm{ZnS}$ reaction products in the PPS-ZnPh interfacial regions; that is, a high conversion rate of $\mathrm{ZnPh}$ as the cycles increased led to the formation of large amount of the $\mathrm{ZnS}$ which has no effect on the cathodic reaction-caused decomposition of the interfacial intermediate 


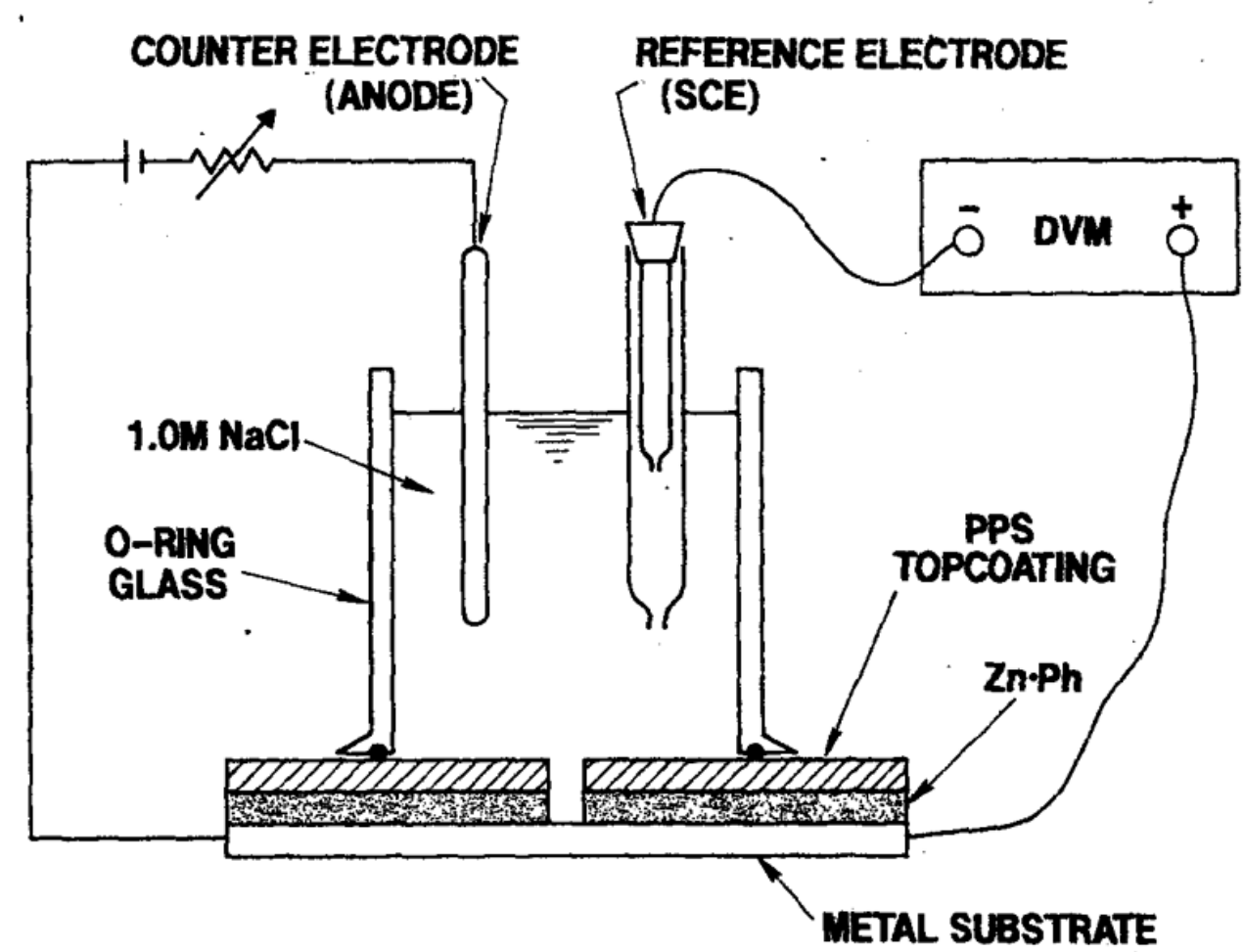

Figure 5. Schematic diagram of the cathodic delamination test.

layers. Because the cathodic corrosion reaction, which occurs under the coating adjacent to the defect creates a high $\mathrm{pH}$ environment at the interfacial boundary, the magnitude of susceptibility of the interfacial intermediate layers to alkali-induced decomposition plays a key role in resisting the cathodic failure. Thus, it is reasonable to assume that the intermediate $\mathrm{ZnS}$ formation, which is insoluble in alkali solution at $\mathrm{pH} \sim 13$, has a high resistance to cathodic reaction-created alkaline environments, thereby resulting in a minimum rate of delamination. This concept directly reflects why the rate of delamination for the control is higher than that of panels after the cycles because of the presence of a large amount of non-reacted $\mathrm{ZnPh}$ layers, which are essentially vulnerable to the alkali-catalyzed dissolution. 


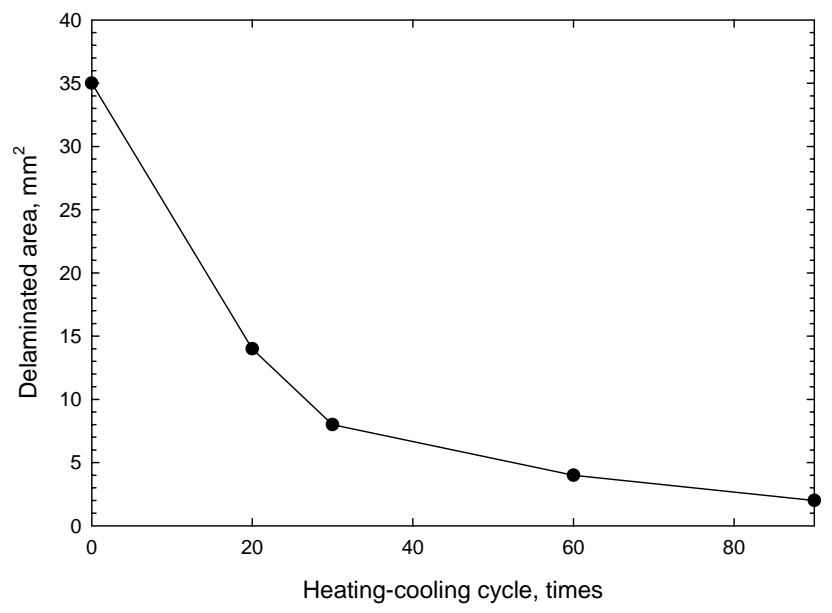

Figure 6. Rate of delamination of PPS coating films from the underlying primed steel after cathodic tests for eight days for the PPS/ZnPh/steel joints as a function of heating-cooling cycles.

\subsection{Thermal Conductivity and Mechanical Properties}

In previous work we demonstrated that incorporating carbon fibers, $\sim 7.5 \mu \mathrm{m}$ in diameter by $~ 3 \mathrm{~mm}$ long, into PPS matrix improved the properties of PPS-based coating materials. Although the maximum amount of these $3 \mathrm{~mm}$ long fibers that could be added to the thermoplastic was only around $1.5 \mathrm{wt} \%$, the thermal conductivity of the PPS was increased by $60 \%$, the tensile strength improved by 2.4 times, and the elongation enhanced 1.5 fold compared with that of the unmodified PPS coating [15]. One major reason for these improvements was the outstanding adherence of the carbon fibers' surfaces to the PPS matrix, reflecting the cohesive failure mode that occurred in the PPS layer. In addition, the PPS itself possessed outstanding hydrothermal stability at temperatures up to $200^{\circ} \mathrm{C}$ and great adherence to $\mathrm{ZnPh}$ primer deposited over the underlying steel surfaces [16]. Thus, this carbon fiber-reinforced PPS composite coating had a high potential for use as a thermally conductive internal liner of carbon steel heat exchanger tubes in geothermal binary-cycle power plants operating at temperatures up to $200^{\circ} \mathrm{C}$.

Recently, advanced milling technology has made it possible to produce an extremely short carbon microfiber while retaining all of these mechanical properties and 
the filament's shape. The milled fiber is $100-200 \mu \mathrm{m}$ long with $7.5 \mu \mathrm{m}$ diameter [17]. One attraction of using such a short fiber is that a substantial amount of it can be incorporated into the PPS. Thus, an emphasis in the current study was directed toward assessing the effectiveness of this milled carbon microfiber in enhancing further the thermal conductivity and the mechanical properties of the carbon fiber-reinforced PPS composite coatings.

\subsubsection{Experimental}

Asbury Graphite Mills, Inc. supplied the milled carbon microfibers (AGM-94). These carbon fibers derived from polyacrylonitrile (PAN) precursor were $7.5 \mu \mathrm{m}$ in diameter and 100-200 $\mu \mathrm{m}$ long. The surfaces of the fibers do not have any sizing materials nor were they treated physically or chemically. The fibers at 1, 2, 3, 4, and $5 \%$ by weight of the total mass of PPS were added to the PPS slurry. Using the same coating process technology as that described in the early experimental work on the adherence of underlying steel, this process was repeated three times to assemble a coating film with a thickness ranging from 100 to $130 \mu \mathrm{m}$.

\subsubsection{Results}

Figure 7 shows a SEM microphotograph of the "as-received" milled fibers. The length of the fibers ranged from 100 to $200 \mu \mathrm{m}$. The SEM close examination (Figure 7, right) of the fibers' surfaces revealed the presence of many gutters (less than $0.5 \mu \mathrm{m}$ wide) oriented along the fibers' axes. The EDX spectrum accompanying this SEM image displayed only one element, carbon, attributed to the fiber. Since the penetration of Xrays from EDX through the solid surface is up to $1.5 \mu \mathrm{m}$ depth, it is possible to assume that the subsurface layer (1.5 $\mu \mathrm{m}$ thick) of the fibers was mostly occupied by carbon. 

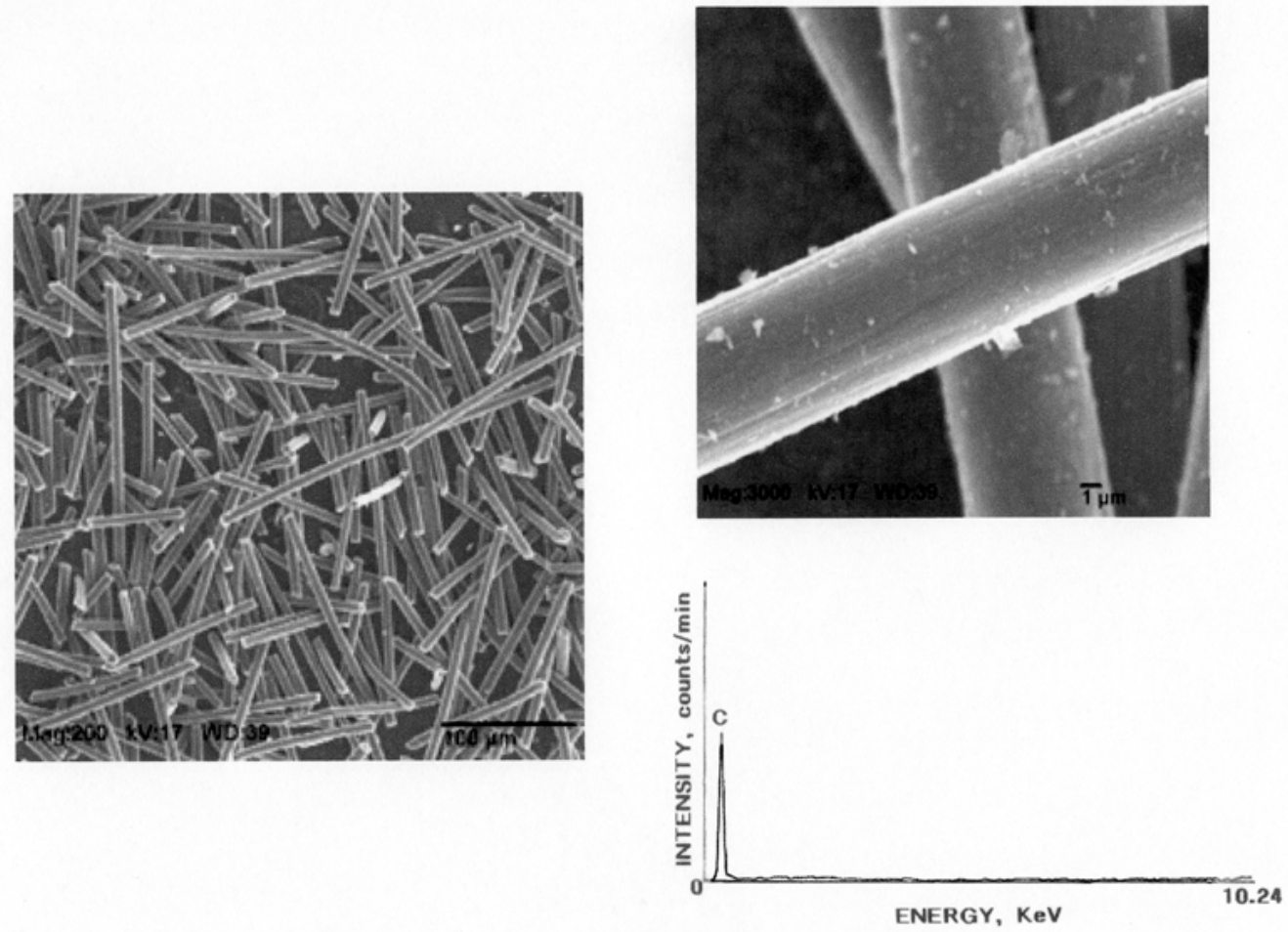

Figure 7. SEM microphotographs and EDX spectrum of “as-received” milled microfibers.

Also, we inspected by XPS the chemical states occupying the fibers' outermost surface sites. The feature of XPS $\mathrm{C}_{1 \mathrm{~s}}$ core-level excitation curve (not shown) closely resembled that taken from $\sim 3$ mm long carbon fibers' surfaces used in our previous study [16]. Namely, the curve encompassed the $C$ in the graphite as the principal component at $284.5 \mathrm{eV}$ and two $\mathrm{C}$ moieties in the $\mathrm{C}=\mathrm{O}$ and $\mathrm{COOH}$ groups as the minor components at 288.0 and $289.2 \mathrm{eV}$, respectively. This information strongly suggested that the superficial layer of fibers has numerous graphitic basal planes, which act as heattransferring components.

Table 1 lists the changes in thermal conductivity and mechanical properties such as tensile strength and elongation of the PPS composite coatings as a function of fiber content. As expected, the thermal transfer efficiency of the PPS was enhanced with increasing fiber content. In fact, the thermal conductivity of the non-reinforced PPS rose 
2.6fold to $1.03 \mathrm{~W} / \mathrm{mk}$ by incorporating $5 \mathrm{wt} \%$ fiber. The fibers contributed significantly to an improvement in the tensile strength of the PPS film. With only $1 \mathrm{wt} \%$ fiber, its tensile strength was $~ 3.2$ times higher than that of non-reinforced films. Further addition of fibers, to $3 \mathrm{wt} \%$, resulted in the development of the highest tensile strength, tantamount to an improvement of $~ 5.2$ times over that of the non-reinforced ones; beyond that content, strength seemed to decline. Correspondingly, the value of $38.2 \mathrm{MPa}$

Table 1. Thermal conductivity and mechanical properties of carbon microfiberreinforced PPS composite films

\begin{tabular}{|c|c|c|c|}
\hline Fiber, wt \% & $\begin{array}{c}\text { Thermal } \\
\text { conductivity, w/mk }\end{array}$ & $\begin{array}{c}\text { Tensile strength, } \\
\text { MPa }\end{array}$ & Elongation, \% \\
\hline 0 & 0.40 & 7.9 & 2.2 \\
\hline 1 & 0.64 & 25.0 & 3.0 \\
\hline 2 & 0.75 & 34.5 & 4.4 \\
\hline 3 & 0.89 & 40.8 & 5.8 \\
\hline 4 & 0.95 & 39.3 & 5.7 \\
\hline 5 & 1.03 & 38.2 & 5.2 \\
\hline
\end{tabular}

for $5 \mathrm{wt} \%$ fiber-reinforced PPS film was $~ 6 \%$ lower than that of the film with 3 wt $\%$ fiber. Since the maximum efficiency of the fiber reinforcement is directly related to the development of high interfacial shear strength at the interface between the fibers and the PPS matrix, the entire surfaces of each one of the multidirectional fibers in the matrix should be completely covered with PPS. Yet, adequate adherence of the fibers' surfaces to PPS is required to allow a crack to propagate through the matrix without significant pulling of the fibers. Based upon this concept, the incorporation of an excessive amount of fibers into the matrix may cause incomplete coverage of fibers' surfaces with PPS, thereby creating a weak interfacial boundary at the fiber/PPS joint. The formation of this weak boundary might result in the development of a low interfacial shear strength. If this interpretation is valid, the decrease in shear bond strength caused by deficient coverage of the fiber's surfaces with PPS may be the reason why the incorporation of $>3 \mathrm{wt} \%$ fibers generated a retrogression of tensile strength. A similar trend was observed in the measurements of elongation, which were taken during tensile failure; namely, the elongation of the PPS films increased with an increasing fiber content up to $3 \mathrm{wt} \%$; 
beyond this amount, its value descended. The inclusion of fibers in the PPS matrix served significantly in enhancing the elastcity of the composite film. In fact, $3 \mathrm{wt} \%$ fiber reinforcement allowed as much as a $5.8 \%$ elongation, corresponding to $\sim 2.6$ times improvement over the non-reinforced PPS films.

Focus now shifted to exploring the microstructure developed in the coating layer and at interfaces between the fibers or zinc phosphate primer and the PPS matrix after exposure for up to 14 days at $200^{\circ} \mathrm{C}$ brine. The results would provide us with important information on whether the fiber-reinforced PPS composite coating layer still retains its ability to protect the underlying steel against corrosion. Figure 8 shows the SEM and EDX analysis for the cross-sectional profile of the $3 \mathrm{wt} \%$ fiber-reinforced PPS composite coating adhering to the zinc phosphate-primed steel after 14 days exposure. The SEM image shows no signals that reflect the failure of the composite coating as a corrosion protective barrier; there is no delamination of the composite layer from the primed steel and no blisters were generated at the interfacial boundary regions between the composite and primed steel. To assess how well the surfaces of fiber adhere to the PPS matrix, the square area in the composite layer was magnified up to ten times. The enlarged image revealed that the excellent adhesive bonding at interfaces between the fiber and matrix remained unchanged.

\subsection{Corrosion-Preventing Performance}

All the information described in 2.2. Thermal Conductivity and Mechanical Properties was correlated directly with the ability of fiber-reinforced PPS composite coatings to protect the underlying carbon steel against corrosion. 


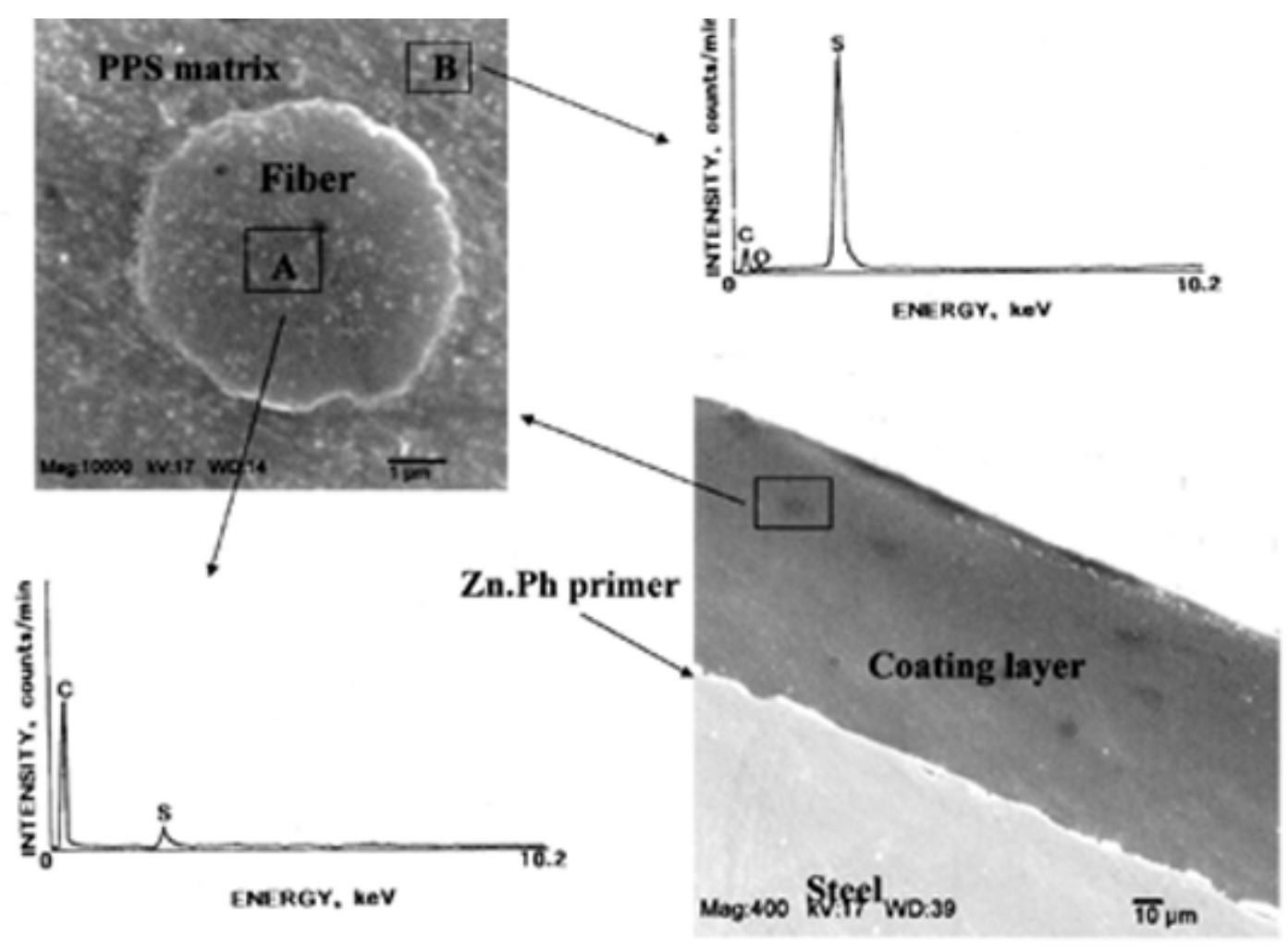

Figure 8. SEM-EDX result for the cross-sectional profile of $3 \mathrm{wt} \%$ fiber-reinforced PPS composite coating deposited on the ZnPh-primed steel after 14 days exposure in $200^{\circ} \mathrm{C} \mathrm{CO}_{2}$-laden brine.

\subsubsection{Experimental}

The coated test panels (63 mm x $63 \mathrm{~mm}$ ) were exposed for up to 14 days in an autoclave containing 20,000 $\mathrm{ppm} \mathrm{CO}_{2}-13 \mathrm{wt} \% \mathrm{NaCl}$ solution at $200^{\circ} \mathrm{C}$. AC electrochemical impedance spectroscopy (EIS) was used to evaluate the ability of the exposed coating films to protect the steel from corrosion. The specimens were mounted in a holder, and then inserted into an electrochemical cell. Computer programs were prepared to calculate theoretical impedance spectra and to analyze the experimental data. Specimens with a surface area of $1300 \mathrm{~mm}^{2}$ were exposed to an aerated $0.5 \mathrm{M}$ sodium chloride electrolyte at $25^{\circ} \mathrm{C}$, and single-sine technology with an input AC voltage of 10 $\mathrm{mV}$ (rms) was employed over a frequency range of $10 \mathrm{KHz}$ to $10^{-2} \mathrm{~Hz}$. To estimate the 
protective performance of the coatings, the pore resistance, $\mathrm{Rp},\left(\mathrm{ohm}-\mathrm{cm}^{2}\right)$ was determined from the plateau in Bode-plot scans that occurred in low frequency regions.

\subsubsection{Results}

Here, focus centered on the magnitude of the ionic conductivity generated by the electrolytes passing through the composite coating layers. EIS tests were conducted on the carbon steel panels coated with 0, 1, 3, 4, and 5 wt\% fiber-reinforced PPS composites, before and after exposure for up to 14 days in the 20,000 $\mathrm{ppm} \mathrm{CO}_{2}$-laden brine solution at $200^{\circ} \mathrm{C}$. For the EIS test, particular attention in the overall impedance curve was paid to the impedance value, called the pore resistance, Rp, which can be determined from the plateau in the Bode-plot features (the absolute value of impedance $|\mathrm{Z}|$, ohm- $\mathrm{cm}^{2}$ versus frequency, $\mathrm{Hz}$ ) occurring at sufficiently low frequencies [18]. A high value of Rp signifies a low degree of penetration of electrolyte into the coating layer, corresponding to an impervious film. Figure 9 plots the pore resistance, Rp, against the exposure time at $200^{\circ} \mathrm{C}$ for fiber-reinforced and non-reinforced PPS composite coatings. Before exposure, the Rp value depended on the fiber content in the composites. The incorporation of 1 and $3 \mathrm{wt} \%$ fibers led to the highest Rp value of $7 \times 10^{11} \mathrm{ohm}-\mathrm{cm}^{2}$, corresponding to an increase of $\sim 17 \%$ over that of the non-reinforced ones. The inclusion of an appropriate amount of fibers seems to densify the composite structure, reflecting a lower rate of ion permeability. However, the composites with a fiber content of $>3 \mathrm{wt} \%$ showed a decline in Rp. With $5 \mathrm{wt} \%$ fibers, the Rp value fell by one order of magnitude to $8.0 \times 10^{9} \mathrm{ohm}$ $\mathrm{cm}^{2}$, compared with that of the $3 \mathrm{wt} \%$ fiber composites. Although we have no experimental evidence, one possible reason may be the air-trapping behavior of the fibers during mixing with the PPS slurry. Trapped air in the slurry can cause the formation of a porous microstructure in the composite coatings. Over14 days of exposure, the Rp values of all the coatings tended to decline, meaning that extended exposures caused the uptake of more electrolytes by the coating layers. 


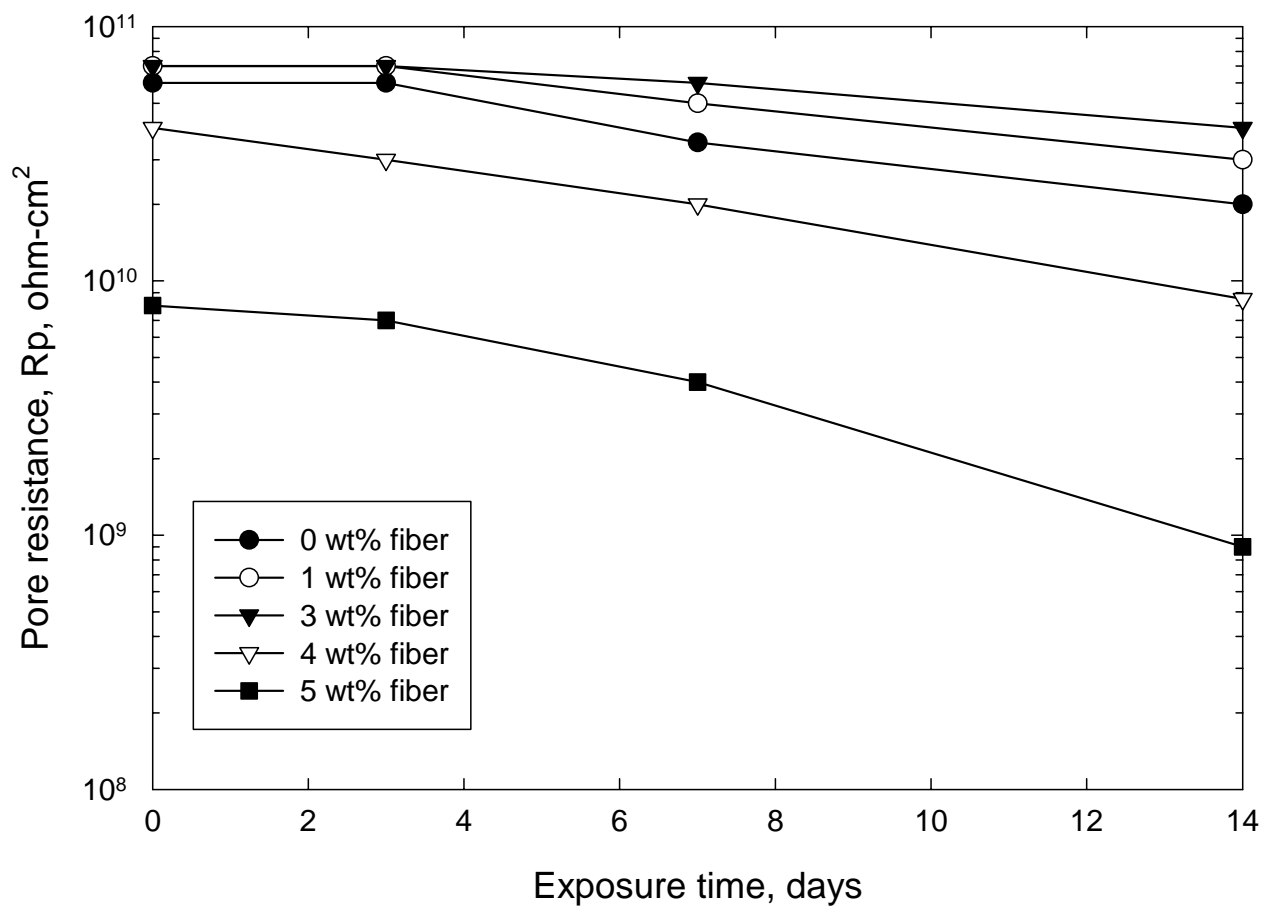

Figure 9 . Changes in Rp for steel panles coated with fiber-reinforced PPS composite and non-reinforced composite as a function of exposure time at $200^{\circ} \mathrm{C}$.

\subsection{Anti-hydrothermal Oxidation}

A short-term exposure test in $200^{\circ} \mathrm{C}$ brine suggested that although PPS coatings offered improved protection of carbon steel against corrosion, compared with the other polymeric coatings, such as epoxy, polyurethane, polyimide, and polyamide, its surfaces underwent a hydrothermal oxidation. Such hydrothermal oxidation involved two critical problems: One was the enhanced rate of permeation of the corrosive electrolytes through the PPS layer during prolonged exposure; and the other issue concerned the changes in topographical surface configuration, implying that the smooth surface texture of the coating was converted into a rough one. A major concern about the latter issue is that a rough surface texture promotes the deposition of scales resulting from the migration of mineral particles from the geothermal brine onto its surfaces. These results drove us to look for way of making PPS more resistant to oxidation by hot brine. One strategy is to 
modify PPS with other thermoplastics, which have outstanding resistance to oxidation, and also provide a surface slip. For this purpose, polytetrafluoroethylene (PTFE) thermoplastic was employed as the additive and blending material for PPS because of its superior thermal and chemical stability, as well as lower surface energy [19].

Emphasis in this present study was directed toward investigating the effectiveness of PTFE additive in alleviating the oxidation of PPS film's surfaces during exposure to a low pH, hypersaline brine at 200EC. The research, therefore, centered on two objectives: One is to gain information on the thermal properties of the PTFE-blended PPS coatings, such as its melting and crystallization temperatures; and, the changes in surface chemical composition before and after exposure in the simulated geothermal brine environment. The other refers to the assessment of the sensitivity of PTFE-blended PPS coating surfaces to silica scaling after exposure them to a $200^{\circ} \mathrm{C}$ sodium metasilicate-dissolved brine of which the silica content was $\sim 11$ times higher than that in geothermal brine.

\subsubsection{Experimental}

PTFE powder (commercial grade, SST-3H), supplied by Shamrock Technologies Inc., was used as a slip-enhancing and oxidation resistant additive to PPS. PTFE-blended PPS powders, with PPS/PTFE ratios of 90/10, 80/20, 60/40, and 40/60 by weight, were prepared in a rotary blender. For comparison, unblended PPS powder was used. The silica-rich brine consisted of $2.0 \mathrm{wt} \%$ sodium metasilicate, $\mathrm{Na}_{2} \mathrm{SiO}_{3} .5 \mathrm{H}_{2} \mathrm{O}, 14.0$ wt\% $\mathrm{NaCl}$, and $84 \mathrm{wt} \%$ water. The coated and non-coated steel panels (13 mm x $77 \mathrm{~mm}$ ) were immersed for up to 7 days in this brine at $200^{\circ} \mathrm{C}$.

\subsubsection{Results}

\subsubsection{Thermodynamic Properties}

Considering the repeated melting-crystallization behaviors of PTFE-blended PPS polymers, the cyclic differential scanning calorimetry (DSC) curves of blended polymers were investigated at a heating-cooling rate of $\pm 10 \mathrm{EC} \mathrm{min}^{-1}$ and at temperatures ranging from 25E to 450EC in air. Samples were prepared as follows: Open aluminum DSC pans were filled with $\sim 5 \mathrm{mg}$ slurries containing three components, the PPS, PTFE, and isopropyl alcohol, and then placed in an oven at 450EC in air for 3 hours. The melted 
samples were subsequently cooled to room temperature at the rate of $-10 \mathrm{EC} \mathrm{min}^{-1}$; the pans were then sealed with aluminum covers. The sealed samples were heated again to 450EC at the rate of $+10 \mathrm{EC} \mathrm{min}^{-1}$ and immediately cooled to 90EC at the rate of - 10EC $\mathrm{min}^{-1}$. Cooling from 450EC to low temperature was accomplished using a DuPont mechanical cooling accessory equipped with a DSC. The unblended PPS polymer was also used as control sample.

The resulting cyclic DSC curves for the PPS/PTFE ratios of 100/0, 80/20, and 60/40 are illustrated in Figure 10. The typical thermodynamic DSC scan (a) for the unblended PPS, denoted as a 100/0 ratio, had endothermic peak at 260EC, reflecting its melting point, Tm1. On cooling the melted polymer, an exothermic peak, Tc, was recorded around $170^{\circ} \mathrm{C}$, which represents the heat evolved during the crystallization of PPS. By comparison, the curve (b) of the blended PPS with 80/20 ratio disclosed two endothermic peaks at 260EC and 320EC. Since the first peak is associated with the melting point of PPS, it is possible to assume that the second one, Tm2, represents the melting point of PTFE. Correspondingly, the appearance of two exothermic peaks at $170 \mathrm{E}$ and $310 \mathrm{EC}$ is reasonably thought to be due to the crystallization point, Tc1 and Tc2, of PPS and PTFE, respectively. When the PPS-to-PTFE ratio was reduced to 1.5, the cyclic curve (c) revealed a conspicuous decrease in intensity of the PPS-related Tm1 and

Tc1 peaks, while a marked growth of Tm2 and Tc2 peaks for PTFE can be seen. This information provides no clear evidence for the formation of reaction products yielded by interactions between the PPS and PTFE in the melting-flowing processes. In other words, if PPS chemically reacts with PTFE, a new peak related to the reaction products should have appeared at a different temperature from these ones. 


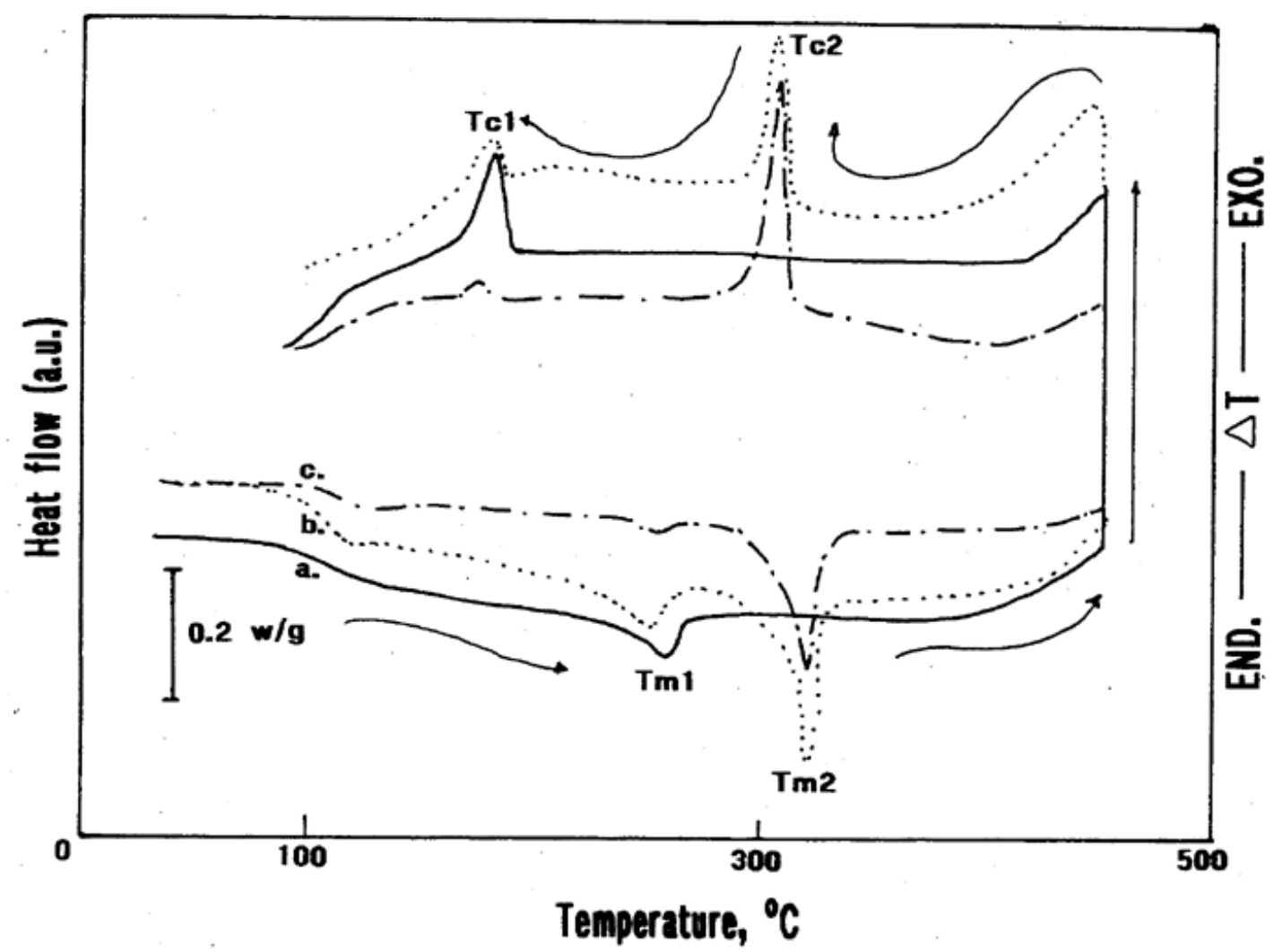

Figure 10. Cyclic DSC curves for polymers with PPS/PTFE ratios of (a) 100/0, (b) 80/20, and (c) 64/40.

\subsubsection{Surface Chemistry}

Focus next centered on surveying the changes in chemical composition and state for the coating surfaces after exposure for 7 days in autoclave containing a low $\mathrm{pH}$, hypersaline brine at 200EC. The fractions of the respective chemical elements were estimated by comparing the XPS $\mathrm{S}_{2 \mathrm{p}}, \mathrm{Cl}_{2 \mathrm{p}}, \mathrm{C}_{1 \mathrm{~s}}, \mathrm{Na}_{1 \mathrm{~s}}$, and $\mathrm{F}_{1 \mathrm{~s}}$ peak areas, which can be obtained from the differential cross sections for core level excitation. All XPS measurements were made at an electron take-off angle of 40E, which corresponds to an electron-penetration depth of $5.0 \mathrm{~nm}$; thus, the XPS data provide the atomic fractions present in the surface layers with a thickness of $5.0 \mathrm{~nm}$. Table 2 gives the XPS atomic fractions of the coating surfaces with the various PPS/PTFE ratios before and after exposure. Before exposure, the chemical composition of blended polymer surfaces with a 90/10 ratio was quite different from that of the unblended ones (100/0 ratio); the surface 
was occupied by two major atoms, the $\mathrm{F}$ and $\mathrm{C}$, while the $\mathrm{S}$ and $\mathrm{O}$ atoms are present as the minor elements. Since the source of F arises from the PTFE, it is possible to assume that the outermost surface site of the coatings was made up almost exclusively by the PTFE polymer. This finding seems to verify that the separation of PTFE from PPS might take place in the blended polymer system, and consequently the surface layer has PTFE as its major component and PPS as its minor one. Incorporating more PTFE into the PPS resulted in the elimination of the $\mathrm{S}$ atom in the surface layer. In fact, no $\mathrm{S}$ atom was detected from the 80/20 ratio coating surfaces. Hence, almost the entire surface is assembled by PTFE because of the presence of $\mathrm{F}$ and $\mathrm{C}$ as the dominant atoms. As expected, the 60/40 and 40/60 ratio coatings had the surface chemical composite similar to that of the $80 / 20$ ratio, excepting the concentration of oxygen present. There is no detection of oxygen in the 40/60 ration coating, seemingly suggesting that the top surface layer was completely constructed by the PTFE.

When these coating surfaces were exposed, a considerable attention was paid to the changes in chemical composition caused by the hydrothermal oxidation of coatings. As is seen, such changes for unblended PPS (100/0 ratio), compared with that before exposure, were as follows; 1 ) the incorporation of considerable amount of oxygen into the surface layers, 2) the marked decrease in the amount of both the S and $\mathrm{C}$ atoms arising from PPS, and 3) the migration of some Na and Clatoms from the brine solution to the surfaces. The most critical issue to be considered was the result (1); namely, the PPS surfaces can undergo oxidation during exposure to a hot brine solution at 200EC. The amount of oxygen after exposure for 7 days raised as much as five times to $26.4 \%$. By comparison with this, blending PTFE considerably reduced the degree of PPS's oxidation. The amount of oxygen for the 90/10 ratio coating was only a $9.4 \%$, corresponding to three times less than that of the 100/0 ratio. The amount tends to decrease with the reduction of PPS/PTFE ratio. Such a trend exemplifies that the incorporation of oxygen into the surface layer depends mainly on the proportion of PTFE to PPS; the coating's surfaces with a 40/60 ratio indicated the presence of very low oxygen content of $0.4 \%$. As a result, blending the PTFE with PPS led to transforming the oxidizable PPS surfaces into antioxidation ones. 
Table 2. Chemical Composition of Coating Surfaces with PPS/PTFE Ratios of 100/0, 90/10, 80/20, 60/40, and 40/60 Before and After Exposure for 7 Days

$\begin{array}{ccrrrrrr}\begin{array}{c}\text { PPS/PTFE } \\ \text { ratio }\end{array} & \begin{array}{c}\text { Exposure time, } \\ \text { Days }\end{array} & \underline{\mathrm{S}} & \underline{\mathrm{Cl}} & \underline{\mathrm{C}} & \underline{\mathrm{O}} & \underline{\mathrm{Na}} & \underline{\mathrm{F}} \\ 100 / 0 & 0 & 13.0 & 0.0 & 81.6 & 5.4 & 0.0 & 0.0 \\ 90 / 10 & 0 & 2.5 & 0.0 & 45.7 & 3.0 & 0.0 & 48.8 \\ 80 / 20 & 0 & 0.0 & 0.0 & 42.3 & 2.1 & 0.0 & 55.6 \\ 60 / 40 & 0 & 0.0 & 0.0 & 43.3 & 0.2 & 0.0 & 56.5 \\ 40 / 60 & 0 & 0.0 & 0.0 & 42.9 & 0.0 & 0.0 & 57.1 \\ 100 / 0 & 7 & 5.3 & 1.2 & 66.1 & 26.4 & 1.0 & 0.0 \\ 90 / 10 & 7 & 0.8 & 0.0 & 42.9 & 9.4 & 0.0 & 46.9 \\ 80 / 20 & 7 & 0.0 & 0.0 & 41.6 & 6.5 & 0.0 & 51.9 \\ 60 / 40 & 7 & 0.0 & 0.0 & 41.9 & 1.2 & 0.0 & 56.9 \\ 40 / 60 & 7 & 0.0 & 0.0 & 41.1 & 0.4 & 0.0 & 58.5\end{array}$

\subsubsection{Inertness to Silica Scale Deposition}

Figure 11 shows the SEM microphotographs coupled with the EDX for the noncoated bare steel surfaces after 1,3 , and 7 days immersion in $200^{\circ} \mathrm{C}$ sodium metasilicatecontaining brine. As seen in the top photograph, the surfaces of steel were covered with a certain amount of silica after only a day of immersion, clearly demonstrating that carbon steel is very sensitive to the deposition of silica. The EDX spectrum accompanying this SEM image had three elements, $\mathrm{O}, \mathrm{Si}$, and Fe. The first two elements are attributable to silica, and the last element arises from the underlying steel. Extending the immersion time to 3 days (middle photograph) resulted in the complete coverage of steel's surfaces by silica as evident from the elimination of the Fe signal in the EDX spectrum. Since EDX provides information on chemical elements existing in the subsurface layer with an $\sim 1.5 \mu \mathrm{m}$ thick, this silica layer covering the steel has the thickness of at least $1.5 \mu \mathrm{m}$. After 7 days immersion, the SEM image revealed the formation of a well-crystallized silica layer. In addition, the silica scales strongly adhered to the steel surfaces; in fact, 

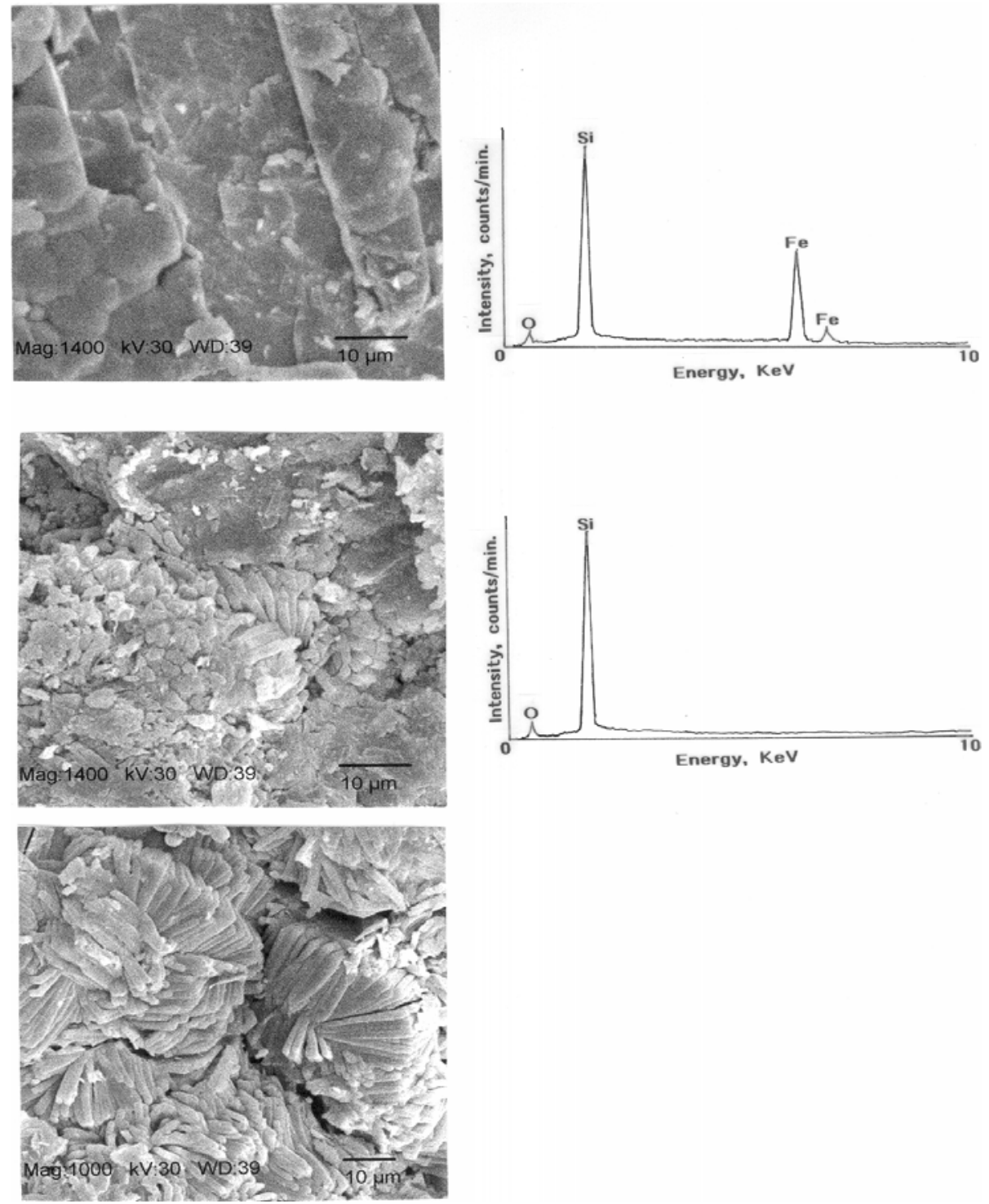

Figure 11. SEM microphotographs coupled with EDX spectra for the surface of carbon steel (top) after 1 day, (middle) 3 days, and (bottom) 7 days immersion in silica-rich brine at $200^{\circ} \mathrm{C}$.

although the steel panels were physically bent at an angle of about 90 degrees, there was hardly any flaking off of scales from the steel's surfaces. As described in our previous paper [4], the principal chemical compound occupying the outermost surface site of the carbon steel was ferric oxide $\left(\mathrm{Fe}_{2} \mathrm{O}_{3}\right)$. Hence, the high sensitivity of steel's surfaces to the silica scaling possible reflects the strong affinity of ferric oxide for silica. However, there is no experimental evidence on how ferric oxide reacts with silica and what kind of the reaction product is formed at the interfaces between them. 
In contrast, coating the steel's surfaces with unblended bulk PPS or PTFEblended PPS dramatically restrained the deposition of silica. Although we observed some silica contaminants on the coating surfaces after 7 days immersion, they were readily removed by rinsing the panels with water. Figure 12 shows the SEM image and the EDX spectrum for the rinsed surfaces of the 7-day-immersed bulk PPS coating. A smooth surface texture is seen, representing the absence of micro-sized silica scales. The EDX spectrum associated with this image included three weak peaks of the $\mathrm{C}, \mathrm{O}$ and $\mathrm{Si}$ elements, together with a prominent signal of S element. Since the C and S elements are attributed to PPS, the other two elements seem to be associated with the $\mathrm{SiO}_{2}$, suggesting that a certain amount of silica still remained over the PPS coating surfaces after rinsing.
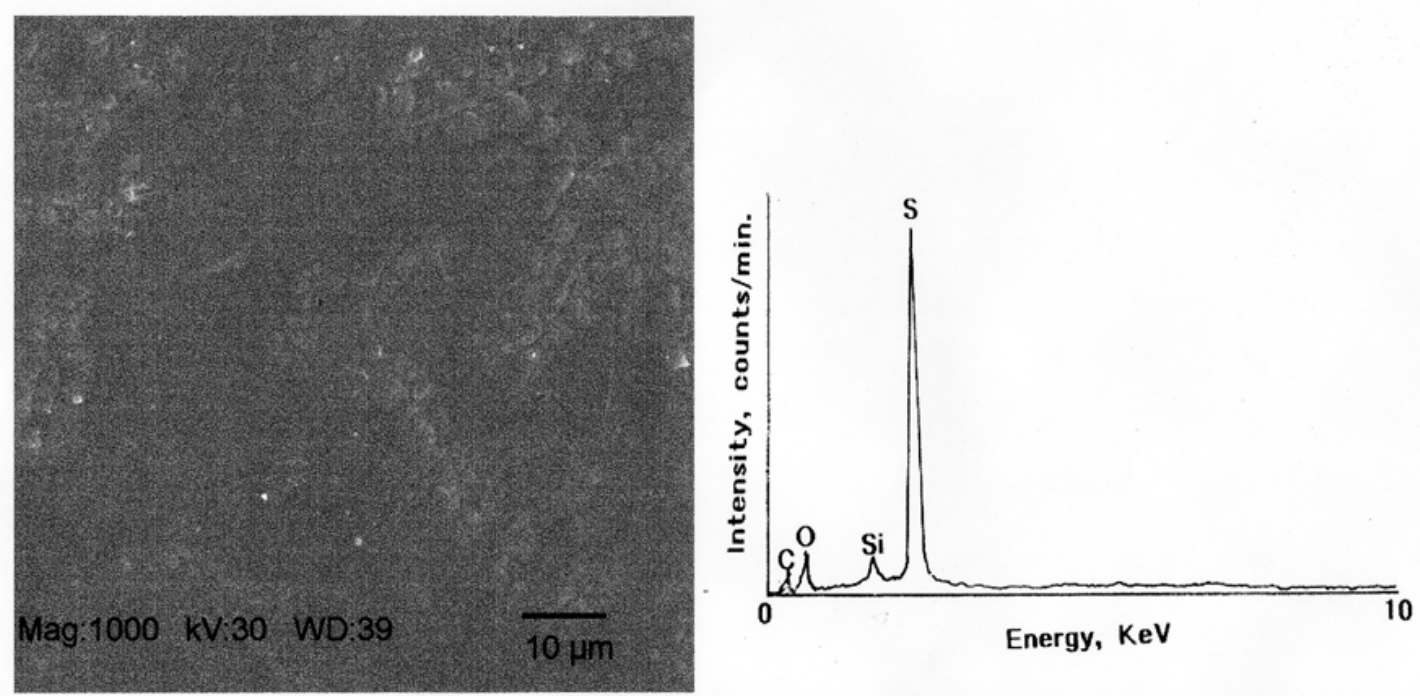

Figure 12. SEM-EDX analyses of PPS coating surfaces after 7 days immersion.

Figure 13 shows the SEM image and EDX spectrum of the surfaces of PTFE-blended PPS coating after 7 days immersion. By comparison with that of the bulk PPS, this image expresses a rough surface texture. However, such a rough microstructure is an inherent characteristic of blended PPS surfaces, and is not due to the attack of the hot brine. The 
EDX spectrum for this subsurface layer with the thickness of $\sim 1.5 \mu \mathrm{m}$ had a prominent $\mathrm{S}$ as the major element and the weak peaks of $\mathrm{C}$ and $\mathrm{F}$ as the minor ones, while the signal of $\mathrm{O}$ element is barely detectable. There is no signal of Si element, implying that the surfaces of PTFE-blended PPS coating are inert to the deposition of silica.
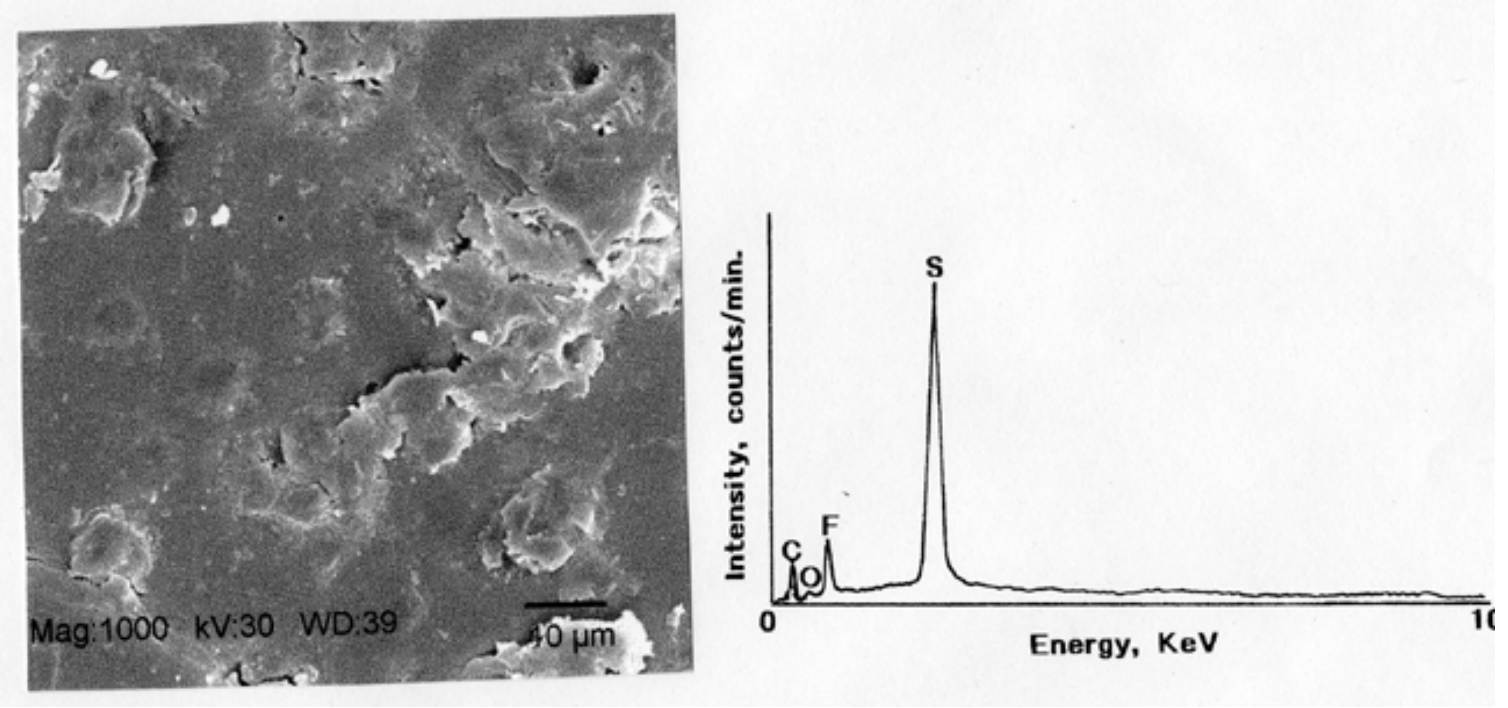

Figure 13. SEM-EDX analysis for PTFE-blended PPS coating surfaces after 7 days immersion.

\subsection{Resistance to Abrasive Wear}

Our field tests at geothermal power plants demonstrate that no matter how and with what the internal surfaces of carbon steel heat exchanger tubes were coated with anti-corrosion and anti-fouling materials, fouling by geothermal brine-induced scale deposits still occurred. Among the technologies used for removing scales, hydroblasting at pressures ranging from 41.3 to $82.7 \mathrm{MPa}$ is commonly employed to clean heat exchanger tubes before their reuse. The major mineralogical constituents of the scales are silicate compounds and silica, raising concerns that when such scale-deposited surfaces are repeatedly cleaned by hydroblasting, the coating's surfaces undergo severe wear and tear. Such damage is due to the bombardment of the surfaces by dislodged hard mineral 
particles under velocity, causing topographical changes from smooth surfaces to rough ones. In the worst case, the liner film was completely worn out, promoting the rate of corrosion of the underlying steel. In addition, the asperity of the coating's surfaces reinforced their strong physical affinity for the scales, thereby making it difficult to dislodge them. Consequently, an adequate resistance to blasting wear is required to extend the coating's useful lifetime as a corrosion-preventing barrier.

In trying to improve the resistance of PPS coating to abrasive wear, the crystalline boehmite engineering ceramic is very attractive for use as the wear-resistant filler because of its great hardness and strength. Recently, advanced nanoscale technology has made it possible to produce a nano-sized boehmite crystal. An attraction in using such extremely fine boehmite filler is that the filled PPS coating's surfaces would offer a sufficient resistance to wear.

\subsubsection{Experimental}

The nanoscale boehmite filler (Catapal ${ }^{\circledR} 200$ Alumina) was supplied by Sasol North American Inc; Table 3 lists some of its chemical and physical properties. Figure 14 shows the secondary electron image of clustered nanosize boehmite filler taken by SEM, and its chemical elements detected from EDX. The boehmite filler at 2, 5, 10, and 15\% by weight of the total amount of PPS was added to the PPS slurry, and the mix was poured into a $110 \mathrm{ml}$ shear blender. Mechanical blending for 2 min converted the agglomerated boehmite powder particles of $\sim 35 \mu \mathrm{m}$ into a primary filler with particles of 100-500 nm; also, this uniformly dispersed the primary fillers in the slurry.

\subsubsection{Results}

The blasting wear resistance of the surfaces of unexposed coatings was assessed using a silica $\left(\mathrm{SiO}_{2}\right)$ grit-blasting hand-held gun with a 2 mm-diameter orifice nozzle. The $\mathrm{SiO}_{2}$ grits (particle size of $15 \mu \mathrm{m}$ ) were conveyed by a compressed air pressure of $0.62 \mathrm{MPa}$ from a backpack hopper to the gun. From a standard distance of $\sim 20 \mathrm{~mm}$, the grits were projected for 2 min onto the coating surfaces at an angle of $\sim 45^{\circ}$. The rate of wear was estimated as the loss in weight of the coatings (mg/min). 
Figure 15 shows the rate of blasting wear for the 2, 5, 10, and $15 \mathrm{wt} \%$ boehmitefilled and unfilled PPS coatings. The data clearly showed that the rate of wear of the

Table 3. Typical chemical and physical properties of “as-received” boehmite filler.

\begin{tabular}{|l|l|}
\hline $\mathrm{Al}_{2} \mathrm{O}_{3}, \%$ & 80 \\
\hline $\mathrm{Na}_{2} \mathrm{O} . \%$ & 0.002 \\
\hline Bulk density, g/l & $500-700$ \\
\hline Surface area (BET), ${ }^{2} / \mathrm{g}$ & 100 \\
\hline Crystalline size, nm & $\sim 30$ \\
\hline Agglomerated particle size, $\mu \mathrm{m}$ & $\sim 35$ \\
\hline
\end{tabular}
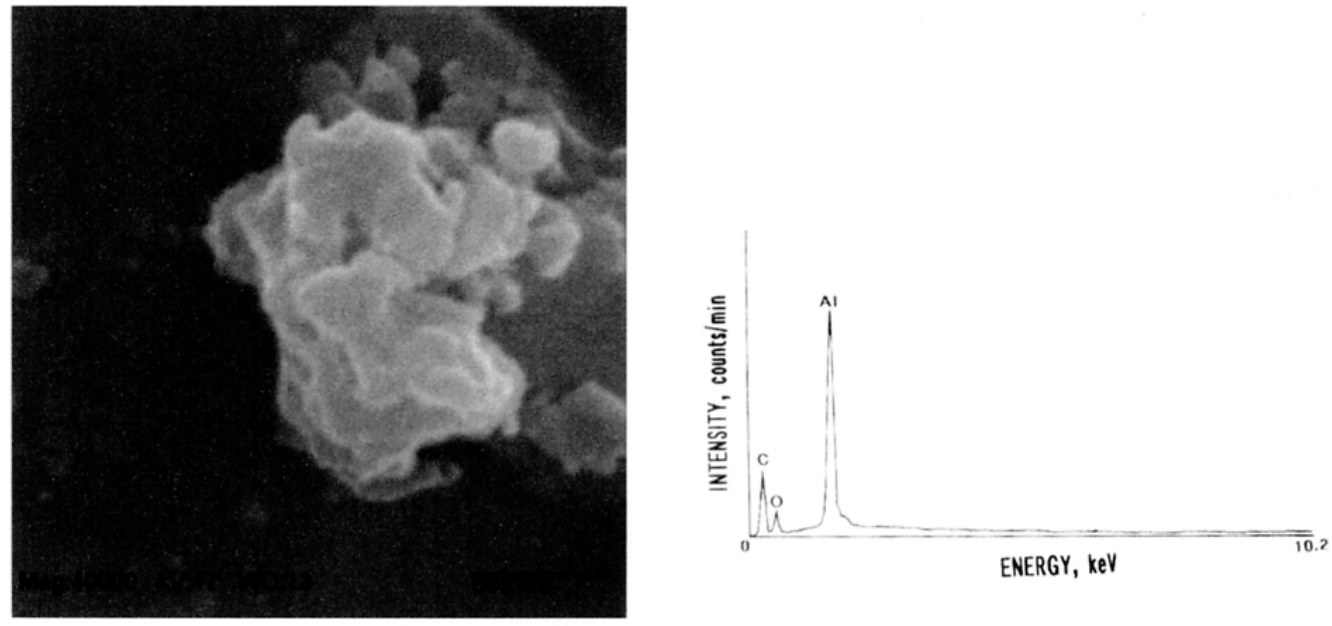

Figure 14. SEM image and EDX spectrum of "as-received” nano-scale boehmite filler's clusters (一: $500 \mathrm{~nm})$.

unfilled PPS coating, at $1.04 \mathrm{mg} / \mathrm{min}$, strikingly decreases with an increasing content of boehmite. When $5 \mathrm{wt} \%$ boehmite was incorporated into the PPS, the rate markedly dropped to $0.35 \mathrm{mg} / \mathrm{min}$, corresponding to three-fold reduction compared with that of unfilled coatings. Further, a reduction to $0.2 \mathrm{mg} / \mathrm{min}$ was obtained by adding $10 \mathrm{wt} \%$; 
beyond this content, the effect of more filler was reduced. Nevertheless, nanoscale boehmite fillers significantly enhanced the resistance of PPS coating's surfaces to blasting wear.

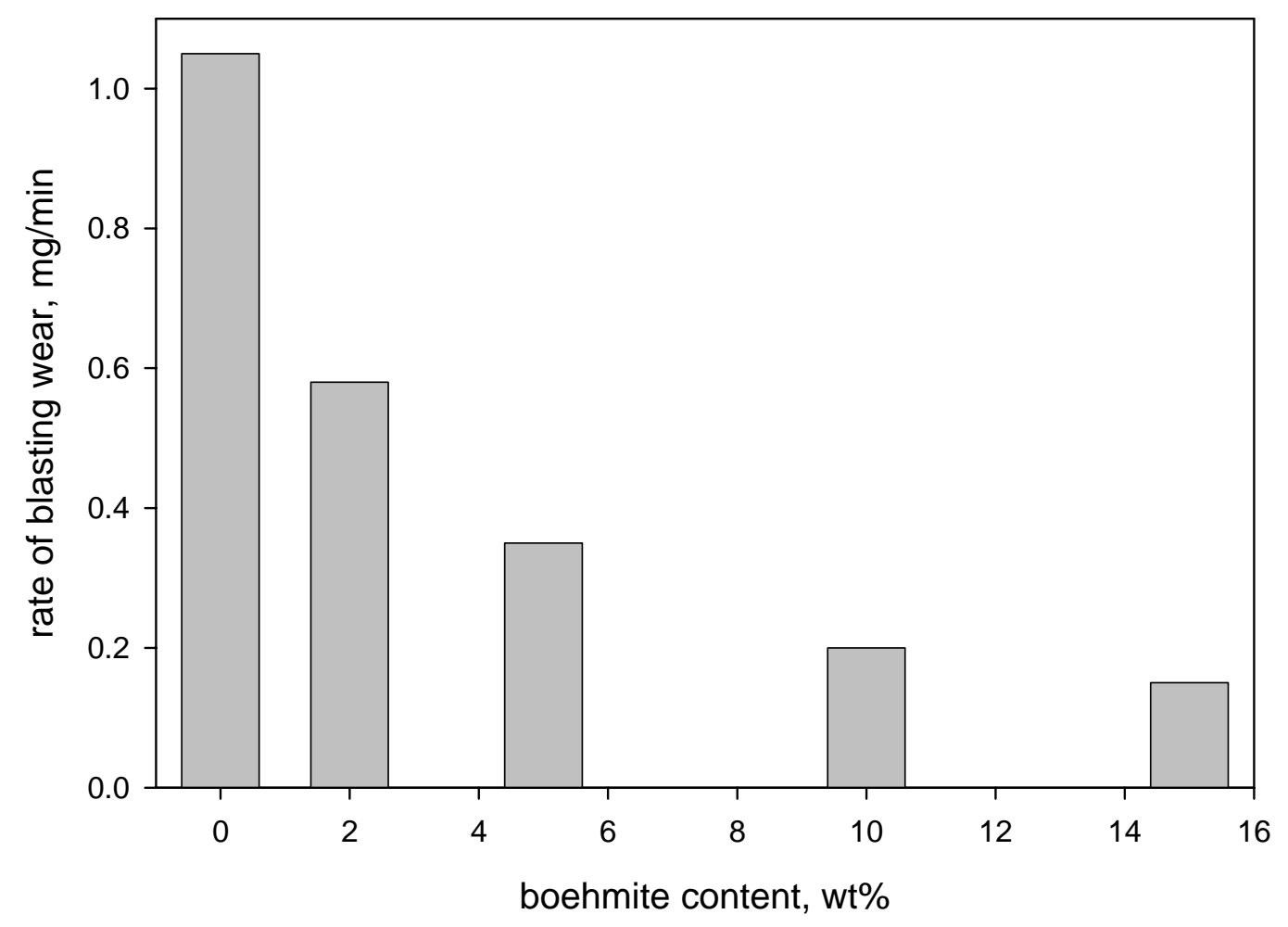

Figure 15. Changes in the rate of blasting wear for PPS coating's surface as a function of nano-scale boehmite filler content.

\subsection{Self-repairing Property}

As described above, the PPS composite material had high potential for use as an internal thermal conductive liner for heat exchanger tubes. However, of particular concern were the micro- and nano-scopic scissions generated in the superficial layer of the composite during operation of the heat exchanger tubes. The development of such cracks as initiators of the propagation of serious damage ultimately diminishes the lining's ability to protect the underlying steel against corrosion, thereby reducing their 
maximum service lifetime as corrosion-preventing barriers. At present, non-destructive techniques such as ultrasonic and radiography can detect failed coating with macroscopic cracks. But, micro and nano-scale damages are too small to be revealed by these technologies. In addition, repairing seriously damaged tubes is a major drawback, because it is very expensive and time consuming. Therefore, the ideal composite liner must have the ability to self-repair the cracks generated in the superficial layer during the service life of tubes in a hydrothermal environment.

Hydraulic inorganic grains, such as calcium silicate $\left(\mathrm{CaO}-\mathrm{SiO}_{2}\right.$ phase system) and calcium aluminate ( $\mathrm{CaO}-\mathrm{Al}_{2} \mathrm{O}_{3}$ phase system) are very attractive for use as self-repairing fillers because their crystals grow after their grain's surfaces came in contact with a hot water. The expanding crystals then may densely fill the open cracks, thereby reconstituting the damaged coatings. As is well documented, in autoclaved $\mathrm{CaO}-\mathrm{SiO}_{2}{ }^{-}$ $\mathrm{H}_{2} \mathrm{O}$ systems, the crystal's features and behavior depended on the $\mathrm{CaO} / \mathrm{SiO}_{2}$ mole ratios; crystals in the ratios of 1.5 to 2.0 , and of 0.8 to 1.3 , respectively, showed fiber-like and needle-like microstructures [20,21]. The size of the crystals ranged from 5 to $20 \mu \mathrm{m}$. On the other hands, hydrated $\mathrm{CaO}-\mathrm{Al}_{2} \mathrm{O}_{3}-\mathrm{H}_{2} \mathrm{O}$ systems were characterized by the rapid growth of flat blade- or plate-like crystals of 10 to $20 \mu \mathrm{m}$ [22,23]. Since a rapid growth of crystals is of important concern to this healing technology, the $\mathrm{CaO}-\mathrm{Al}_{2} \mathrm{O}_{3}$ system filler was selected in this study.

Based upon the information described above, the emphasis of the present study was on assessing the potential of the $\mathrm{CaO}-\mathrm{Al}_{2} \mathrm{O}_{3}$ system in healing and repairing the cracks generated in a superficial layer of PPS coating. The factors to be assessed included the alterations in microstructure at the coating's surfaces after healing, the phase composition of crystals growing in the spaces of the cracks, the ability of the healed material to inhibit corrosion of the underlying steel substrates. All the findings were integrated to elucidate the mechanisms of self-healing and -repair in exposed PPS coatings containing hydraulic inorganic fillers.

\subsubsection{Experimental}

Calcium aluminate (CA, Secar 71) grains, $<40 \mu \mathrm{m}$ in size, used as the $\mathrm{CaO}-\mathrm{Al}_{2} \mathrm{O}_{3}$ system filler material, were obtained from the Lafarge Aluminate Corp. The chemical 
constituents of Secar 71 consisted of $70.2 \% \mathrm{Al}_{2} \mathrm{O}_{3}, 28.6 \% \mathrm{CaO}, 0.9 \% \mathrm{SiO}_{2}$, and $0.3 \%$ $\mathrm{Fe}_{2} \mathrm{O}_{3}$. The X-ray powder diffraction (XRD) data showed that the crystalline components of CA consist of two major phases, monocalcium aluminate $\left(\mathrm{CaO} . \mathrm{Al}_{2} \mathrm{O}_{3}\right)$ and calcium bialuminate $\left(\mathrm{CaO} \cdot 2 \mathrm{Al}_{2} \mathrm{O}_{3}\right)$, and one minor phase, gehlenite $\left(2 \mathrm{CaO} \cdot \mathrm{Al}_{2} \mathrm{O}_{3} \cdot \mathrm{SiO}_{2}\right)$. CA fillers at 2, 5, and $10 \%$ by weight of the total amount of PPS were added to the PPS slurry, and then the CA-filled slurries were deposited on the $\mathrm{ZnPh}$ primed metal panel (62.5 mm x $62.5 \mathrm{~mm}$ ). The average thickness of coating films for 0, 2, 5 and10 wt\% CA was $110,110,100$, and $90 \mu \mathrm{m}$, respectively.

To prepare cleaved PPS coatings, an initial crack $\sim 5 \mu \mathrm{m}$ in wide was cut at the edge of the coated panel's surface using a sharpened diamond blade under a loading of $1.5 \mathrm{~kg}$. Then, the loaded blade was shifted slowly from one edge to other edge of the panel until the length of the cleavage reached $62.5 \mathrm{~mm}$ (Figure 16). The eleven slices were made with the intervals of $5 \mathrm{~mm}$ between the grooves. Scanning electron microscopy (SEM) for the cross-sectional profile of the cleaved coatings revealed that these grooves ranged in depth from $\sim 8$ to $\sim 17 \mu \mathrm{m}$. The grooved coating panels then were exposed for up to 20 days in $200^{\circ} \mathrm{C} 40,000 \mathrm{ppm} \mathrm{CO}$-laden brine (13 wt\% sodium chloride).

\subsubsection{Results}

\subsubsection{SEM-EDX Analyses}

The first experiment focused on visualizing how the CA fillers virtually sealed the cracks in the coatings. To obtain this information, we explored alterations in the microstructure of the cleaved coating surfaces with and without the $5 \mathrm{wt} \%$ CA fillers after a $24 \mathrm{hr}$-exposure in a $200^{\circ} \mathrm{C}$ autoclave. Figure 17 shows the SEM microphotographs in coupling with the EDX spectrum for the CA-filled coating surfaces before and after exposure. For the non-exposed coating (Figure 17, top), the SEM image revealed the microstructure of an $\sim 5.3 \mu \mathrm{m}$ wide cleavage on the coating's surface. The EDX spectrum taken from the area "A" at $~ 2 \mu \mathrm{m}$ depth from the edge of cleavage included a prominent signal from sulfur (S) as the principal element, strong secondary signals from aluminum 


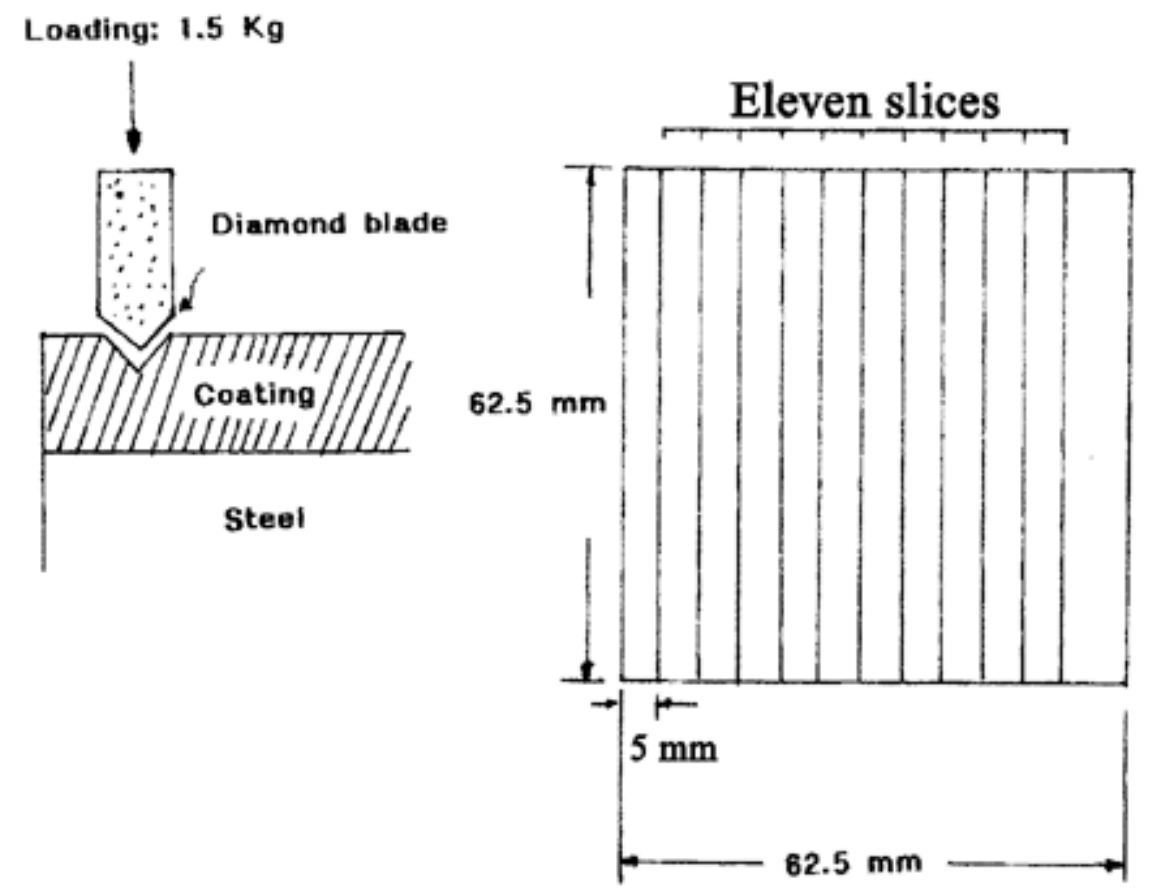

Figure 16. Sample preparation of cleaved PPS coatings deposited on carbon steel panels and the size of specimens for AC electrochemical impedance spectroscopy (EIS) test.

(Al) and calcium (Ca) elements, and lesser signals from carbon (C) and oxygen (O). Both the $\mathrm{S}$ and $\mathrm{C}$ elements appear to arise from the PPS coating, while $\mathrm{Al}, \mathrm{Ca}$, and $\mathrm{O}$ are attributed to the CA fillers. A dramatically changed SEM image was observed on the surface of 24 hr-exposed coating; namely, the entire space in the groove was completely filled up and sealed with block-like crystals (size, $\sim 7 \mu \mathrm{m}$ ) growing on the groove's foundation walls. The EDX spectrum of the crystals denoted as site "C" strikingly differed from that of the unexposed groove at site " $\mathrm{A}$ ”; in particular, the $\mathrm{Al}$ elemental signal had become one of the major peaks coexisting with $S$, while the signal intensity of Ca was markedly reduced. This finding suggests that the major chemical constituent of the crystals is an Al-enriched oxide compound. The other elements, such as $\mathrm{Na}$ and $\mathrm{Cl}$, detected in the crystals, come from the brine. The spectrum of side " $\mathrm{B}$ ", which represents 


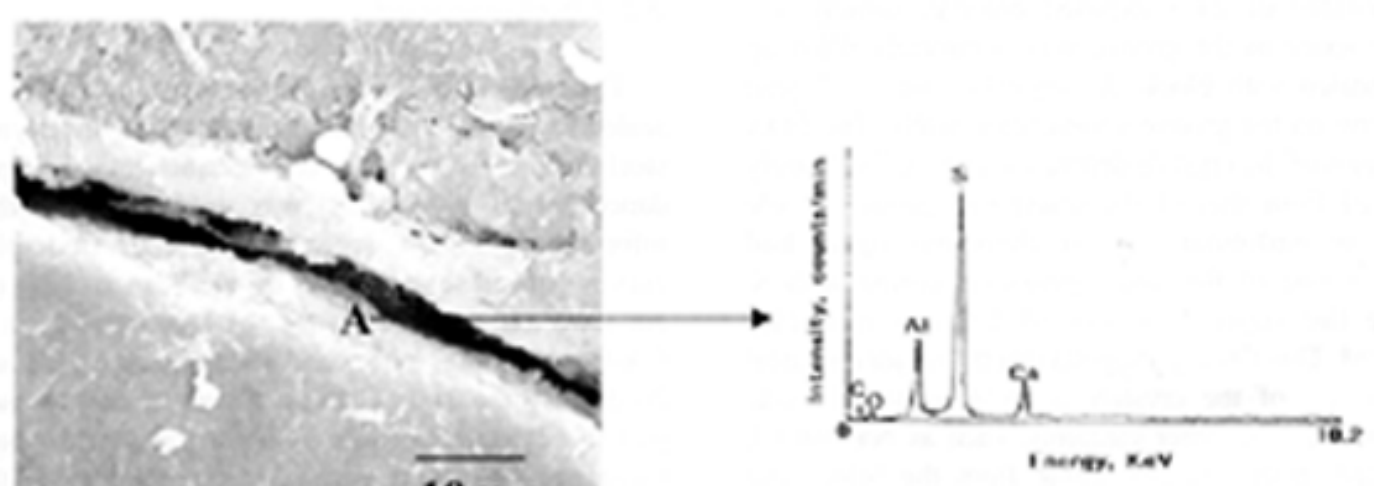

$10 \mu \mathrm{m}$
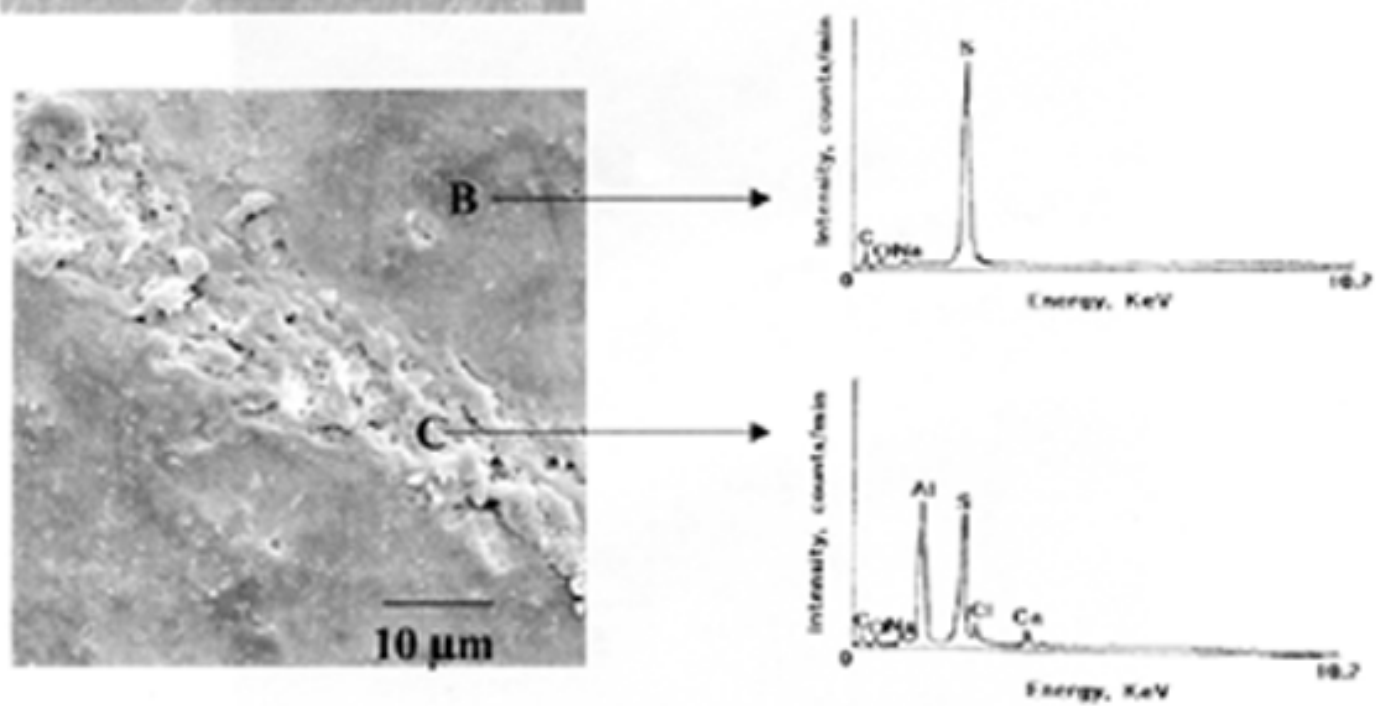

Figure 17. SEM images coupled with EDX spectra for cleaved PPS coatings with 5 wt \% CA fillers before (top) and after (bottom) exposure for 24 hours to $\mathrm{CO}_{2}$-laden brine at $200^{\circ} \mathrm{C}$.

the coating's surfaces, had a pronounced peak of $\mathrm{S}$ and very weak signals of $\mathrm{C}, \mathrm{O}$, and $\mathrm{Na}$. There were no signals related to the CA fillers, reflecting their coverage with a PPS layer of at least $1.5 \mu \mathrm{m}$ because the penetration depth of $\mathrm{x}$-ray from the EDX is $\sim 1.5 \mu \mathrm{m}$.

\subsubsection{EIS Measurement}

The study then shifted to assessing the ability of sealed crystalline materials to protect the underlying steel against corrosion. AC electrochemical impedance 
spectroscopy (EIS) was used to obtain this information. Figure 18 depicts the overall Bode-plot curves [the absolute value of impedance $|\mathrm{Z}|\left(\mathrm{ohm}-\mathrm{cm}^{2}\right.$ ) vs. frequency $(\mathrm{Hz})$ ] for the blemished $5 \mathrm{wt} \%$ CA-filled coatings before and after exposure for 1 and 20 days to the $200^{\circ} \mathrm{C}$ brine. Our attention focused particularly on impedance value in terms of the pore resistance, Rp. This can be determined from the plateau in the Bode plot occurring at a sufficiently low frequency of $1 \times 10^{-1} \mathrm{~Hz}$ for the steel panels coated with $5 \mathrm{wt} \% \mathrm{CA}$ filled PPS. Before exposure to brine, the Rp value for the blemished coatings was $7 \times 10^{6}$ $\mathrm{ohm}-\mathrm{cm}^{2}$. This value raised nearly two orders of magnitude to $3.0 \times 10^{8} \mathrm{ohm}-\mathrm{cm}^{2}$, when the coating was exposed for 1 day. Since the Rp value reflects the magnitude of ionic conductivity generated by the electrolyte $(\mathrm{NaCl})$ passing through the coating layer, a high Rp value means a low degree of permeation of the electrolytes into the coating films. Hence, exposure to the hot brine led to the conversion of the failed coating responsible for a high rate of uptake of corrosive electrolytes into a reconstituted coating that minimized its uptake. This finding strongly demonstrated that the crystals packing the spaces in the cracks significantly contribute to restraining the penetration of electrolytes through the repaired areas. In other words, the sealing effect of crystals accelerated rapidly in a very short period of time, resulting in the recovery and restoration of the corrosion-preventing barrier, which had failed due to cleavages. The reconstituted coatings after 24 hours exposure again were immersed in hot brine for a 20-day-extended exposure. This test was carried out to evaluate whether the crystals would continue to maintain the integrity of the impermeable corrosion-preventing barrier. As seen in figure 18 , the Rp value of the 20-day-exposed coatings was almost the same as that after a 1day-exposure, verifying that the efficacy of closely packed crystals in reducing the permeability of electrolytes in the self-healed coating layer remained unchanged for 20 days. 


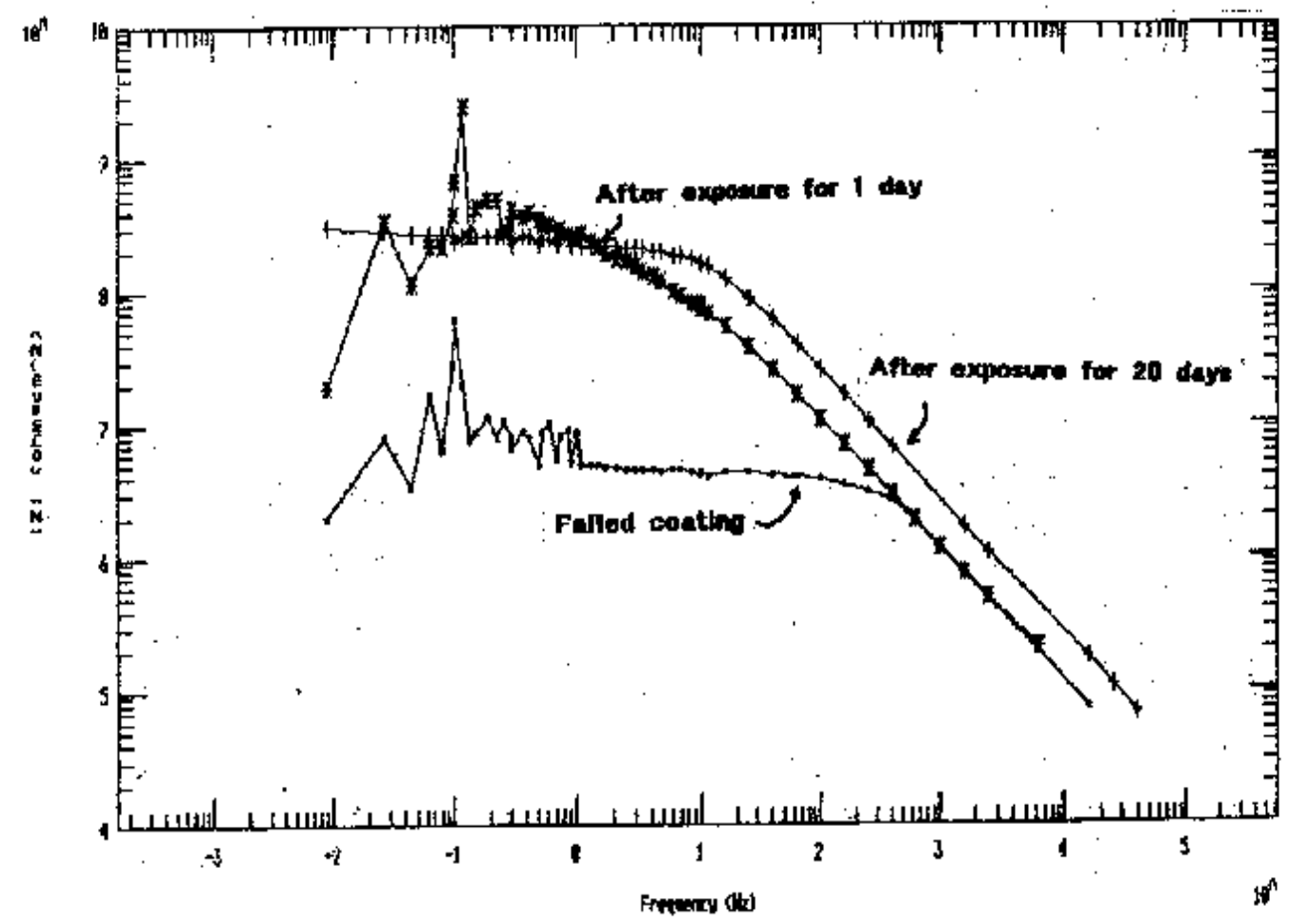

Figure 18. Comparison of Bode-plots for cleaved PPS coatings with 5 wt $\%$ CA fillers before and after exposure for 1 and 20 days.

\subsubsection{XRD Analysis}

Next, the focus concentrated on identifying the phase composition of the crystals contributing to the self-healing and -repair of the coatings by XRD. To increase the amount of XRD-detectable crystals, we added five additional slices to the eleven slices made originally on the $5 \mathrm{wt} \% \mathrm{CA}$-filled coating's surfaces. The coatings then were exposed for $0,2,10$, and 24 hours in $\mathrm{CO}_{2}$-laden brine at $200^{\circ} \mathrm{C}$. Figure 19 compares the XRD features over the diffraction range 0.4436-0.2627 nm. For the unexposed coatings denoted as " 0 " hour, the XRD tracing revealed the presence of three major crystal phases, semi-crystalline PPS, $\mathrm{CaO} \cdot \mathrm{Al}_{2} \mathrm{O}_{3}$ and $\mathrm{CaO} \cdot 2 \mathrm{Al}_{2} \mathrm{O}_{3}$ along with one minor phase related to gehlenite $\left(2 \mathrm{CaO} \cdot \mathrm{Al}_{2} \mathrm{O}_{3} \cdot \mathrm{SiO}_{2}\right)$. Except for the PPS, the other crystalline phases 


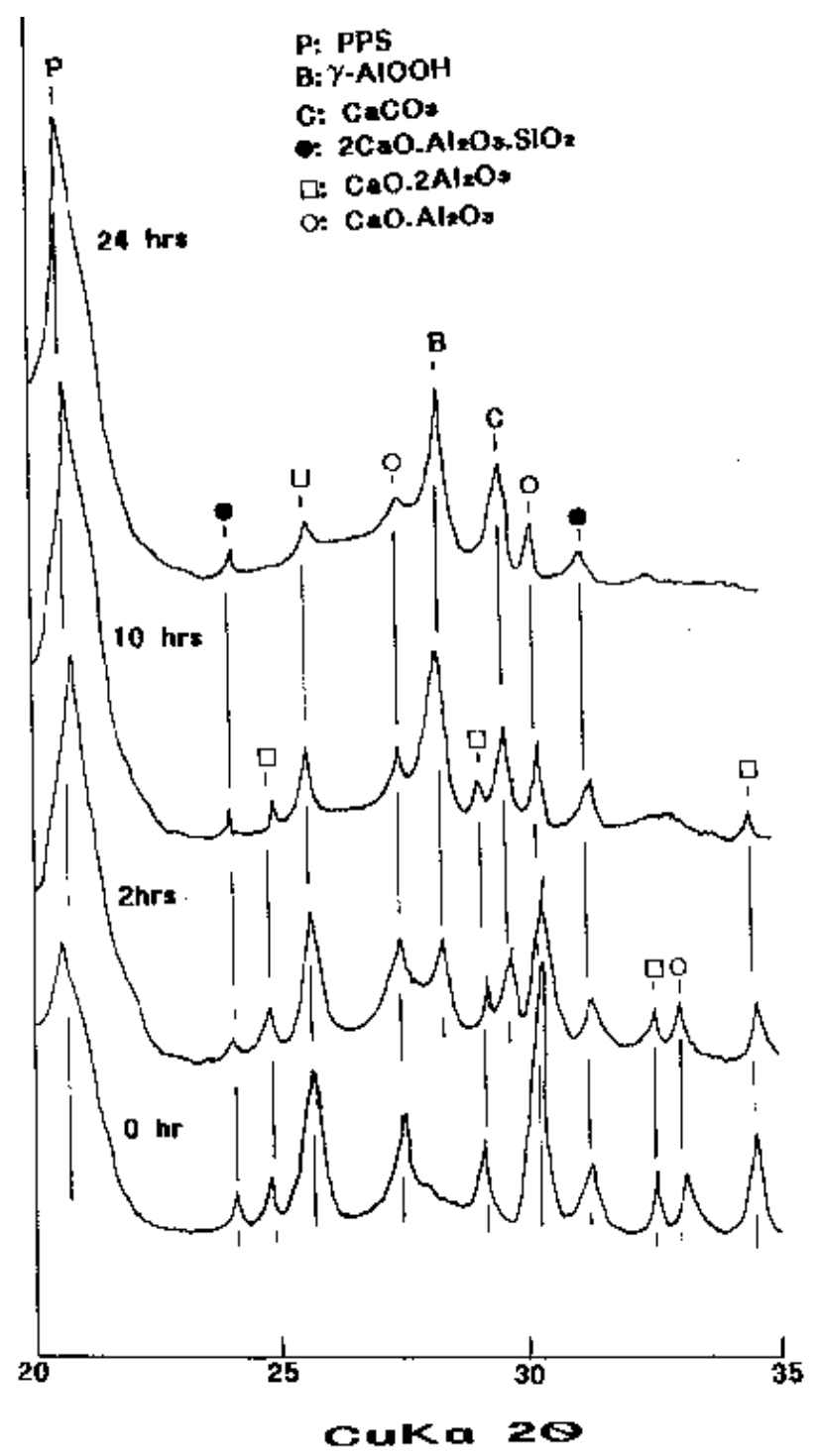

Figure 19. XRD patterns for cleaved 5 wt $\%$ CA-filled PPS coatings before and after exposure for 2,10 , and 24 hours.

are originated from the CA fillers. After 2 hours exposure, XRD pattern was somewhat different from that of the unexposed one. Among the differences were the appearance of two new additional d-spacing lines attributed to the boehmite $(\gamma-\mathrm{AlOOH})$ and calcite $\left(\mathrm{CaCO}_{3}\right)$, and the attenuation of line intensity related to the $\mathrm{CaO} \cdot \mathrm{Al}_{2} \mathrm{O}_{3}$ and $\mathrm{CaO} \cdot 2 \mathrm{Al}_{2} \mathrm{O}_{3}$ phases. After 10 hours, a further attenuation for these CA-associated phases was observed, while increasing the line intensity of the boehmite and calcite phases as the hydrothermal reaction products. After 24 hours, the pattern's feature was similar to that of the 10-hour-exposed coating, excepting that some $\mathrm{CaO} .2 \mathrm{Al}_{2} \mathrm{O}_{3}$-related lines were 
eliminated. Thus, these reaction products appear to be formed within a short exposure period of 2 hours. The data also showed that there are no significant changes in the line shape of the $2 \mathrm{CaO} \cdot \mathrm{Al}_{2} \mathrm{O}_{3} \cdot \mathrm{SiO}_{2}$ phase after exposure, compared with that before exposure. Since calcite is formed by the carbonation of CA fillers, we believed that two reactants in the $\mathrm{CA}, \mathrm{CaO} . \mathrm{Al}_{2} \mathrm{O}_{3}$ and $\mathrm{CaO} \cdot 2 \mathrm{Al}_{2} \mathrm{O}_{3}$, in the $\mathrm{CA}$ preferentially react with $\mathrm{CO}_{2}$ in the brine to form the calcite, rather than $2 \mathrm{CaO} \cdot \mathrm{Al}_{2} \mathrm{O}_{3} \cdot \mathrm{SiO}_{2}$. The transformation of $\mathrm{CaO} \cdot \mathrm{Al}_{2} \mathrm{O}_{3}$ and $\mathrm{CaO} .2 \mathrm{Al}_{2} \mathrm{O}_{3}$ into the calcite and boehmite reaction products perhaps took place through the following reaction pathways:

Hydrolysis of reactants;

$\mathrm{CO}_{2}+\mathrm{H}_{2} \mathrm{O} \rightarrow \mathrm{HCO}_{3}^{-}+\mathrm{H}^{+}$,

$\mathrm{CaO} . \mathrm{Al}_{2} \mathrm{O}_{3}+4 \mathrm{H}_{2} \mathrm{O} \rightarrow \mathrm{Ca}^{+}+2 \mathrm{Al}(\mathrm{OH})_{4}^{-}$,

$\mathrm{CaO} .2 \mathrm{Al}_{2} \mathrm{O}_{3}+7 \mathrm{H}_{2} \mathrm{O}+2 \mathrm{OH}^{-} \rightarrow \mathrm{Ca}^{2+}+4 \mathrm{Al}(\mathrm{OH})_{4}^{-}$,

Interactions;

$\mathrm{Ca}^{2+}+\mathrm{HCO}_{3}^{-} \rightarrow \mathrm{CaCO}_{3}+\mathrm{H}^{+}$,

$\mathrm{Al}(\mathrm{OH})_{4}{ }^{-}+\mathrm{H}^{+} \rightarrow \mathrm{AlH}(\mathrm{OH})_{4} \rightarrow \gamma-\mathrm{AlOOH}+2 \mathrm{H}_{2} \mathrm{O}$.

In the induction stage of the reactions described as hydrolysis of both of environmental $\mathrm{CO}_{2}$ and the $\mathrm{CA}$ filler reactants, $\mathrm{CO}_{2}$ reacts with water to form weakly ionized carbonic acid $\left(\mathrm{HCO}_{3}{ }^{-} \mathrm{H}^{+}\right)$. Meanwhile, the $\mathrm{CaO} \cdot \mathrm{Al}_{2} \mathrm{O}_{3}$ and $\mathrm{CaO} \cdot 2 \mathrm{Al}_{2} \mathrm{O}_{3}$ phases dissociate into two counter ionic species, $\mathrm{Ca}^{2+}$ and $\mathrm{Al}(\mathrm{OH})_{4}^{-}$. Afterward, the $\mathrm{Ca}^{2+}$ begins to interact with the $\mathrm{HCO}_{3}{ }^{-}$counter ion to yield calcite that is one of the reaction products, in the following interaction stage. On the other hand, the uptake of $\mathrm{H}^{+}$by $\mathrm{Al}(\mathrm{OH})_{4}{ }^{-}$generates amorphous $\mathrm{AlH}(\mathrm{OH})_{4}$ as the intermediate derivative. Then, the dehydration of $\mathrm{AlH}(\mathrm{OH})_{4}$ leads to its phase transition into crystalline $\gamma$-AlOOH. Furthermore, the relative intensity of $\gamma$ AlOOH line for the coatings after more than 10 hours exposure was stronger than that of calcite, verifying that the $\gamma-\mathrm{AlOOH}$ is the principal reaction product. A possible explanation of why calcite becomes the secondary reaction product may be due to its susceptibility to reactions with carbonic acid, thereby yielding water-soluble calcium bicarbonate, $\mathrm{CaCO}_{3}+\mathrm{H}_{2} \mathrm{CO}_{3} \rightarrow \mathrm{Ca}\left(\mathrm{CaCO}_{3}\right)_{2}$. This finding is supported by the results from SEM-EDX analysis earlier; namely, the EDX from the crystals filling the grooves revealed a dominant signal from Al. Hence, this dominant element is attributable to $\gamma$ $\mathrm{AlOOH}$. We note that the feature of the XRD pattern from 20-day-exposed coatings is 
almost the same as those from samples exposed for one day (data not shown). This information demonstrated that once the crystalline reaction products were formed in the first 24 hours exposure, there was no further phase transformation of these reaction products caused by extending the exposure to 20 days.

Nevertheless, the agglomeration of well-grown dense boehmite crystals, known as a strong, hard engineering ceramic, in the grooves appears to play the major role in reducing the extent of permeation of the electrolytes, thereby leading to the repair of the cleaved coatings.

\subsection{Field Validation Tests}

Over the past eight years, BNL had fabricated more than thirty liners for 40- and 20ft.-long HX tubes, and National Renewable Energy Laboratory (NREL) had conducted the short-and long-term field validation tests of these liners at the Mammoth Power Plant, California, and Puna Power Plant, Hawaii. Among a large number of field tests, below describes the most recent test results.

Thus, this objective is to evaluate BNL-developed thermally conductive material systems for use as the anti-corrosion and anti-fouling liners of carbon steel heat exchanger (HX) tubes. It also aims to develop lining technologies that confer the maximum corrosion- and fouling-preventing performance of the candidate material systems, and can be scaled up. The approach to evaluating them includes field exposure tests of lined 20-ft.- and 40-ft.-long HX tubes using a BNL-designed state-of-the-art lining apparatus at the Mammoth and Puna geothermal power plant sites in collaboration with NREL and the private sectors; post-test analyses of these materials are undertaken after the field validation tests. The results from latter provide the information on the improvements in the material's formulation and placement technology.

\subsubsection{Full-scale Lining Technologies}

BNL had designed and installed the state-of-the-art lining apparatus that make it possible to complete the entire fabrication process including preparing the internal surface of the tube and depositing the ZnPh primer and PPS-based lines for the 0.97-in.- 
diameter x 20- and 40-ft.-long HX tubes. Using this apparatus, the priming and lining processes were performed in the following sequences.

Surface Preparation of the Internal Surfaces of the Tubes

Step 1: Two 40-ft.-long tubes encased in a $1 / 2$ in. thick urethane slip-on insulation sheath were set on both sides of a galvanized steel truss bed (40-ft-long x 14-in.-wide x 2-ft.-high). A plastic hose was connected from one tube to a pump outlet, while a 6-ft.long hose was attached to the other tube to return cleaning fluid, zinc phosphate make-up solution, and rinsing water to the process tanks or the wastewater drum (Figure 20, left). Meanwhile, the end of each tube was connected with plastic tube (Figure 20, right). This configuration allowed us to circulate the cleaning fluid and a zinc phosphate solution through the inside of the two connected tubes.

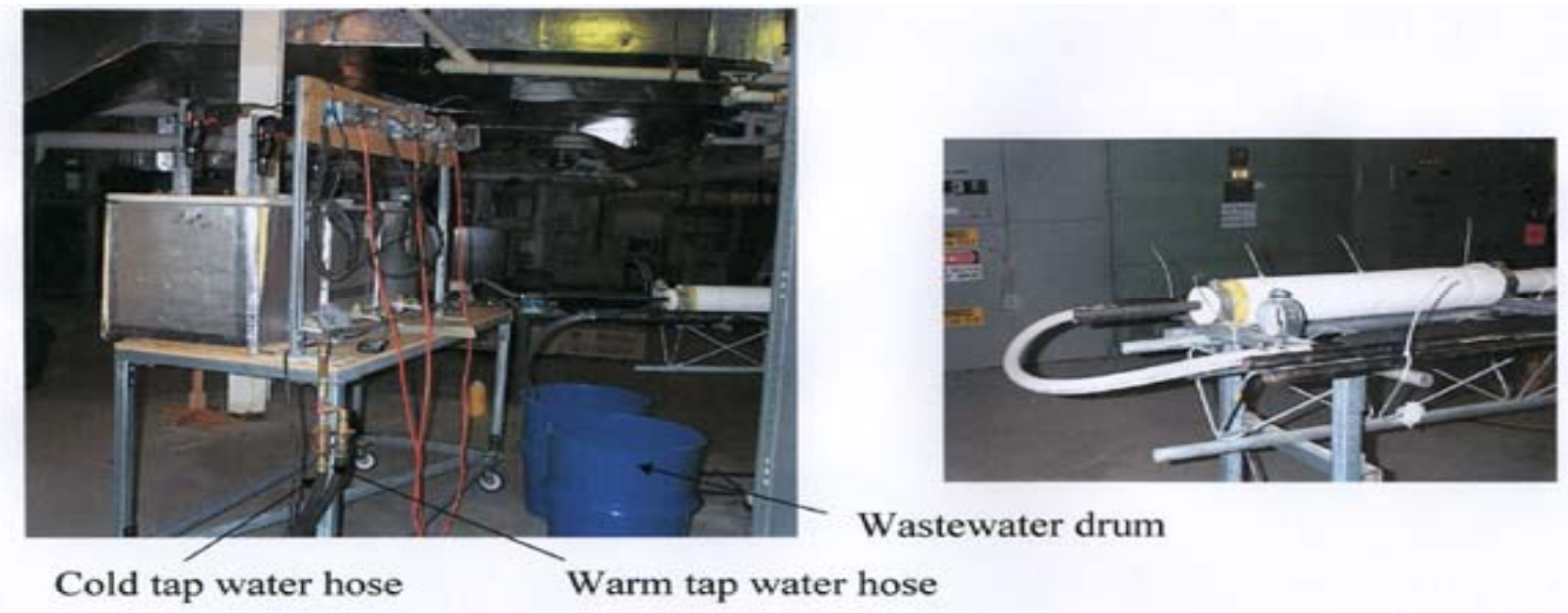

Figure 20. Cold-and warm-tap water hoses connected to circulating pump outlet (right), and plastic tube linked between two HX tube ends (left).

Step 2: A warm water-rinsing hose was connected from the building's hot water system to the manifold (Figure 20, left).

Step 3: Each of three 20-gallon heating tanks was filled with the requisite amount of three different solutions: an alkali cleaning fluid in the first tank; pickle conditioner in 
the second tank; and, zinc phosphate in the third tank. The solutions were agitated with a stirrer while being heated to $75^{\circ} \mathrm{C}$ (Figure 21).

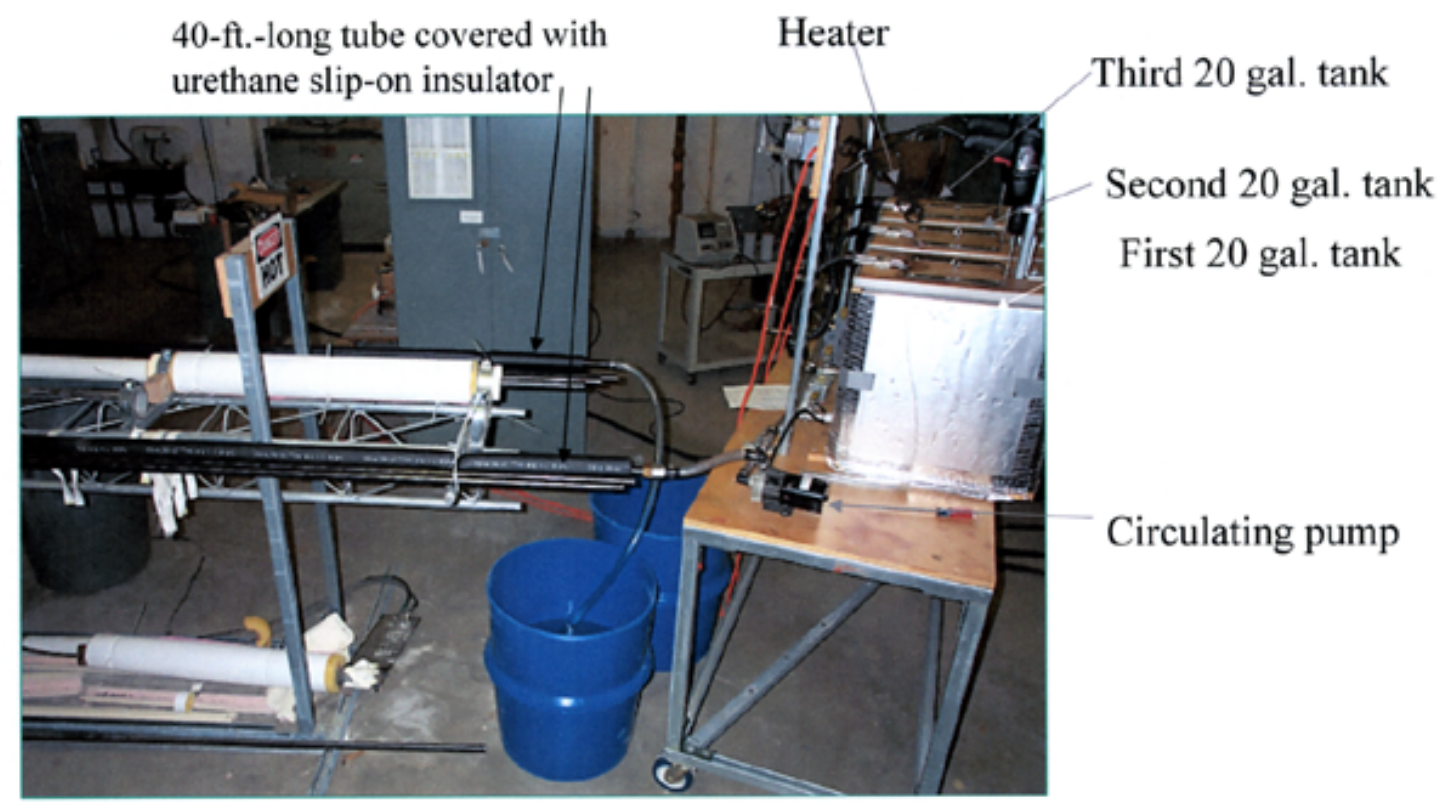

Figure 21. Three 20-gallone tanks, the first containing alkaline cleaner, the second containing pickle conditioner, and third with zinc phosphate primer make-up solution.

Step 4: The warm tap water valve was opened to allow water to run through the tubes for five minutes to preheat them.

Step 5: The valve connected to the first tank containing the alkali-cleaning fluid was opened and the circulating pump is turned on, while the circulating hose connected to one tube was immersed in the first tank (Figure 22). After circulating the cleaning solution for two minutes, the pump and tank valves were shut off, and then the cool tap water valve was opened to push back the cleaning fluid into the first tank. After most of the fluid had been returned to the tank, the circulating hose was shifted from the first tank to the wastewater drum, allowing rinsing water to run through the tubes to remove any remaining alkali cleaner from the tube's internal surfaces. After rinsing for one minute, 
the cooling tap water valve was turned off, and the rinsing water left in tubes was drained out into wastewater drum.

Step 6: Next, the circulating hose was placed in the second tank containing the pickle conditioner, and then the conditioner was circulated for five minutes. Afterwards, the tubes were rinsed in the same process as described in step 5.

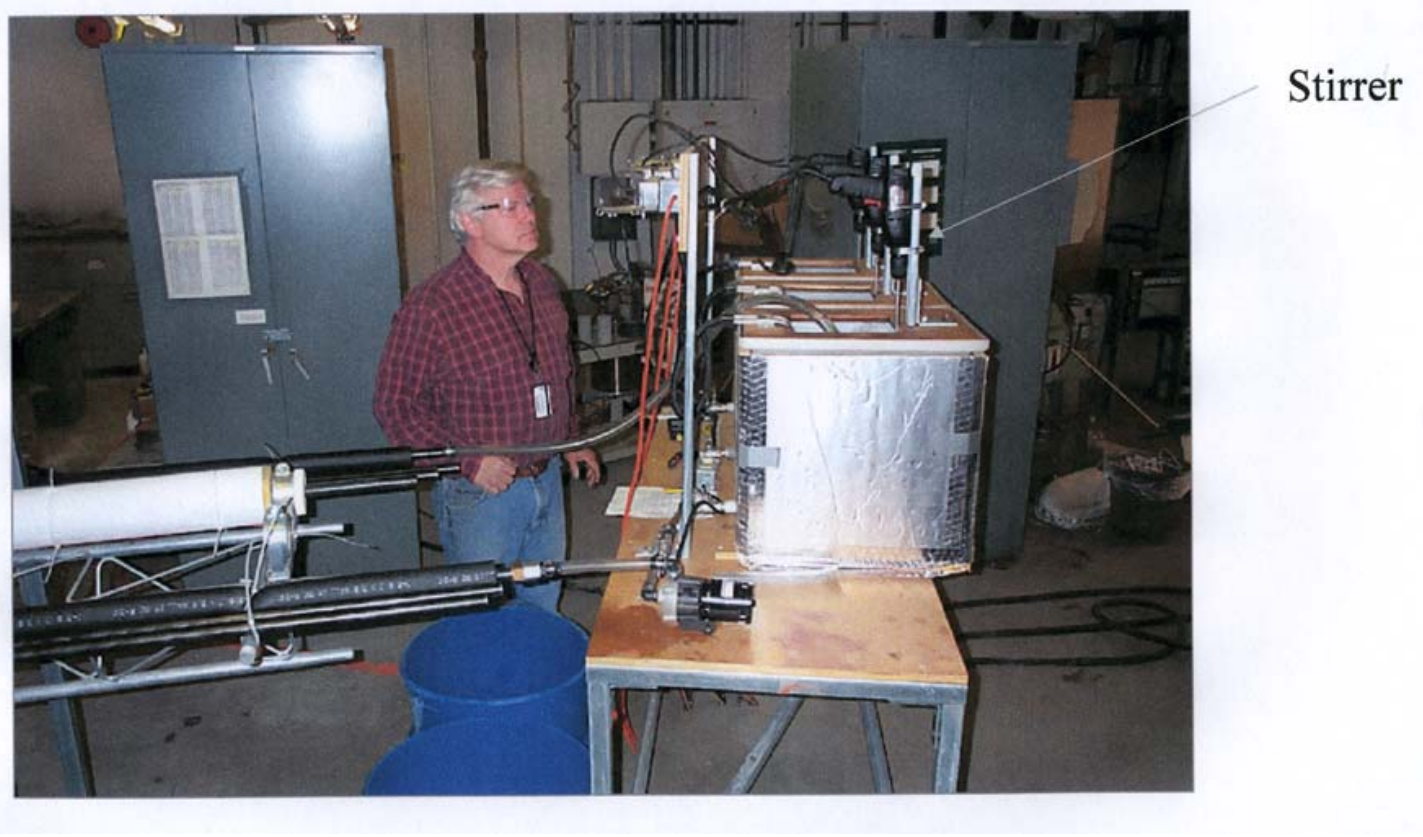

Figure 22. Alkali-circulating cleaning process at $75^{\circ} \mathrm{C}$ for “as-received” $\mathrm{HX}$ tubes.

\section{Zinc Phosphate Primer}

Step 7: Once the preparation of the internal surfaces of the tubes was completed, the circulating hose was immersed into the zinc phosphate make-up solution in the third tank, and then this solution was pumped and circulated for 20 min to deposit the crystalline zinc phosphate primer over the tube’s inner surfaces. Again, the primed surfaces were rinsed with water to eliminate any slag left behind during the zinc phosphate conversion process.

Step 8: After the zinc phosphate primer was deposited, the circulating hose connected to the circulating pump was removed, and then a short piece of plastic tubing was jointed to one end of the HX tube. The other end of the insulated HX tube, still set on 
the truss bed was elevated by a hand hoisting device until there was $\sim 40$ degree angle between the truss bed and the horizontal position. A wet/dry shop vacuum cleaner then was attached to the lower end of the tubes and run for ten min, sucking any residual water out of the tubes. After drying the zinc phosphate-primed tube, the truss bed was returned to the horizontal position.

\section{PPS-based Lining}

Step 9: One of two zinc phosphate-primed HX tubes was set on center of the truss bed, and covered with a fiber-glass thermal insulator. A temperature-limit controller was clamped to the HX tube at its near center point. Six thermocouples were attached at both ends of the tube and at intervals of $10 \mathrm{ft}$. from the tube's ends to monitor heat distribution and the increasing rate of temperature over the entire tube. One of three impedance heaters was connected to the tube's center point (Figure 23).

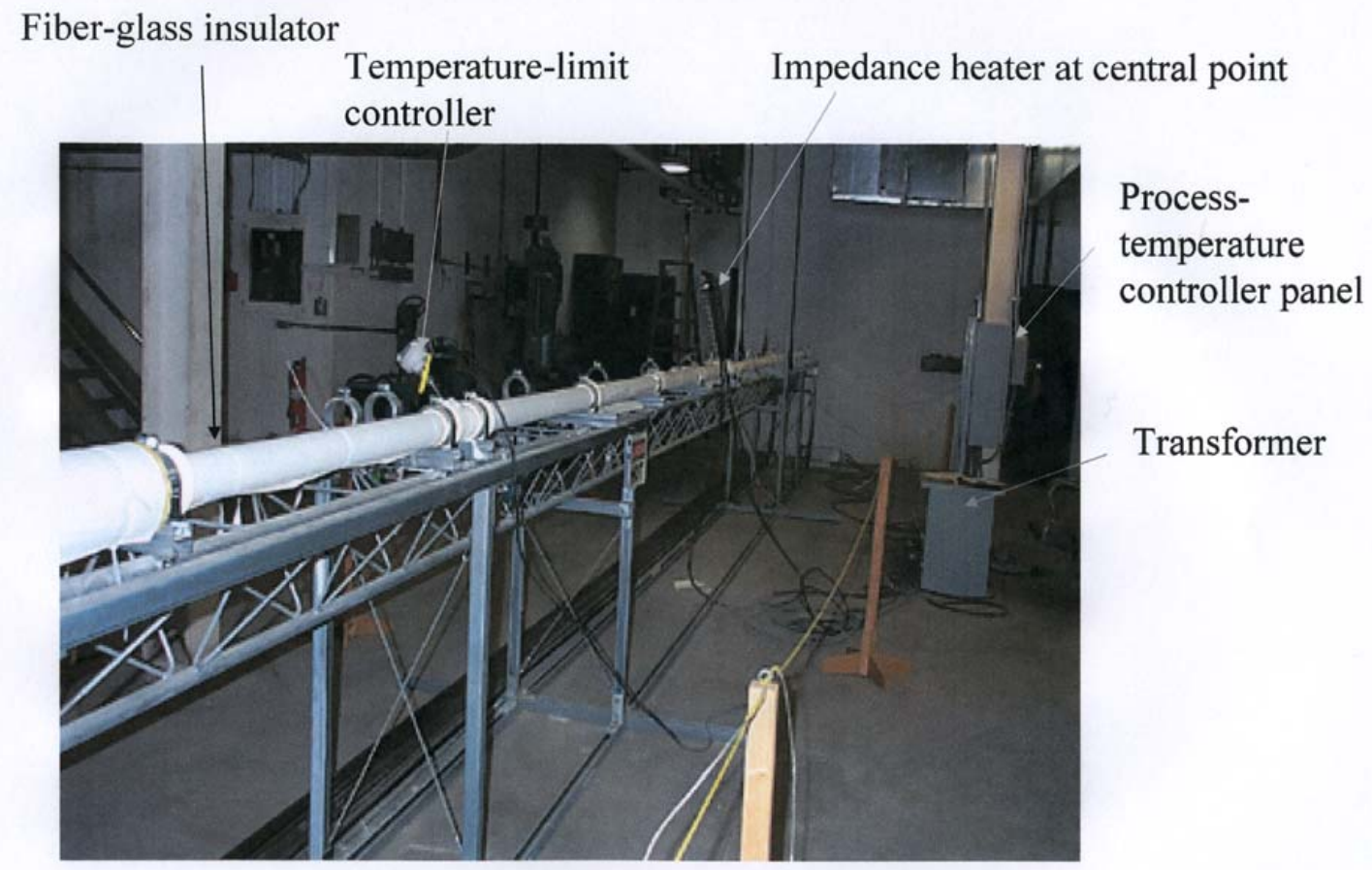

Figure 23. Temperature-limit controller and the one of impedance heaters being set on the primed HX tube is covered with fiber-glass insulator. 
Step 10: One end of the truss bed was elevated by a hand hoisting device until the angle between the truss bed and the horizontal attained $\sim 40$ degree. A funnel was attached to the higher end, while a short length of plastic tubing with a closed ball valve was connected to the lower end. With the operator standing on a ladder, the PPS slurry was slowly poured through a funnel into the tube until it was filled (Figure 24).

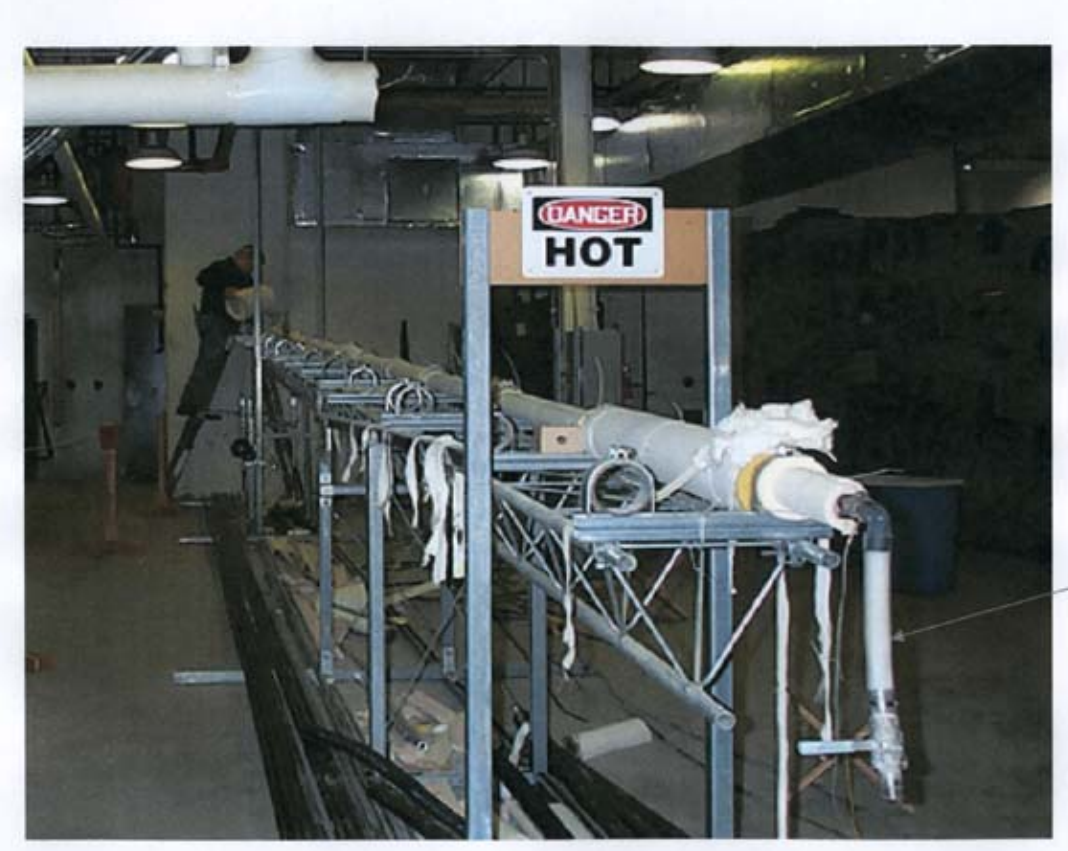

PPS slurry drain tube at lower end of the Primed HX tube

\section{Figure 24. PPS slurry drain tube joined to the lower end of the primed HX tube.}

Step 11: The ball valve on the lower end was opened to allow any extra PPS slurry to drain back into the original container. The slurry-wet tube then was rotated back and forth through 180 degrees by grasping the impedance heater connected to a center point of the tube. This rotating action was repeated five times at intervals of two minutes to prevent excess slurry accumulating in the lower radius of tube, and to ensure an even thickness of the lining. The truss bed then was returned to its horizontal position and again rotated several times for the next five minutes.

Step 12: Two 6-ft. extension elbow tubes were attached to both of the tube's ends. These extension tubes were designed to provide an equivalent temperature throughout the 
entire 40-ft.-long tube being lined by the PPS slurry. The remaining two impedance heaters were attached to these extension tubes (Figure 25).

Step 13: The slurry-lined tube was left overnight to ensure the complete evaporation of all the isopropyl alcohol in the slurry.

Step 14: The impedance heaters at the three different locations were turned on via the process temperature controller set at 23 volts, $25 \%$ output (heat-increase rate of $1.7^{\circ} \mathrm{C} / \mathrm{min}$ ). Almost one hour afterwards, the temperature of the entire tube reached $127^{\circ} \mathrm{C}$. The controller then was set at $55 \%$ output (heat-increase rate of $\sim 7^{\circ} \mathrm{C} / \mathrm{min}$ ). Thirty minutes later, the tube's temperature reached $338^{\circ} \mathrm{C}$. The tube was left at this temperature for two hours to complete the melt-flow of the PPS.

Step15: After two hours, the heaters were turn off. The tube then was left for overnight at room temperature to convert the molten PPS phase into its solid phase.

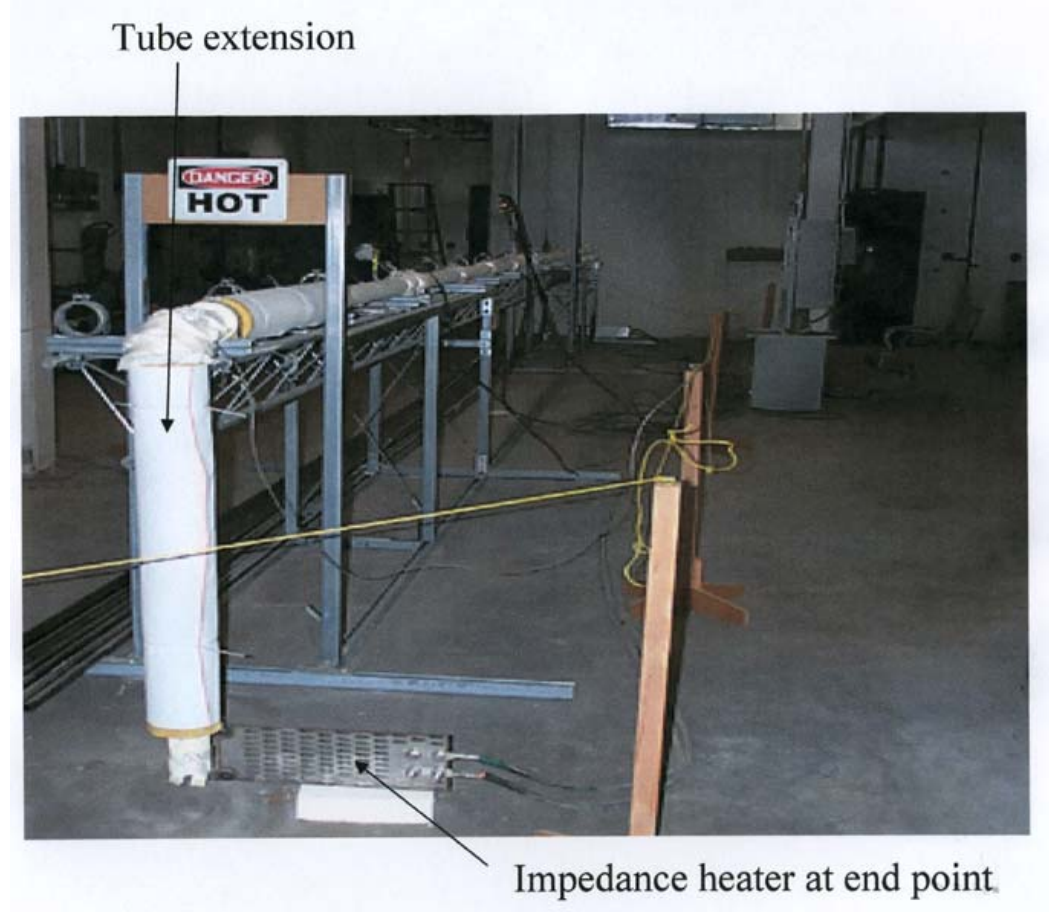

Figure 25. A 6-ft. extension elbow tube linked to the tube's end with an impedance heater attached to the end of the extension tube. 


\subsubsection{Two-year Long Field Exposure Test at Mammoth Power Plant}

As described above, BNL designed and developed several PPS-based coating systems in geothermal material research program aimed at extending the useful lifetime of carbon steel heat exchanger tubes used in geothermal binary-cycle power plants. The coating systems were required to have the following properties: (1) good thermal conductivity; (2) protection of steel tube against corrosion and oxidation; (3) anti-fouling characteristics; and, (4) resistance to abrasive wear. In this field test at the Mammoth power plant, California, operating at temperatures up to $160^{\circ} \mathrm{C}$, the silicon carbide (SiC) grit instead of employing carbon fiber was used as the thermally conductive filler and was packed into the PPS layer. SiC improves heat transfer by $16 \%$, compared with that of unfilled PPS.

In readily transferring the heat energy generated by passing hot brine through the heat exchanger tubes, the coating's surfaces inevitably must possess anti-fouling characteristics. The scales that accumulate over the tubes' surfaces cause a loss in two important functions, the flow pressure of brine and the efficacy of heat transfer, in the heat exchangers. To restore these functions, the scale layers must to be scoured off from the tubes' surfaces. Among the technologies for removing scales, hydroblasting is most commonly used to clean the fouled tubes. However, the strong bond formed between the scales and the tubes' surfaces not only requires highly pressurized hydroblasting, but also a substantial amount of time to dislodge them completely. Such scouring is timeconsuming and very costly. Finally, the coating's surfaces also must be resistant to abrasive wear to abate damage from scratching brought about by the passage of fine hard mineral particles through the tubes, and by the impact of scale particles during hydroblasting. In trying to alleviate the wear damage, we incorporated nanoscale boehmite crystals as the wear resistant filler into the PPS matrix.

To ensure that the laboratory's results are duplicated in the field, BNL lined 20-ft.long carbon steel HX tubes with two different PPS-based coating systems. One system consisted of the $\mathrm{ZnPh}$ primer, the SiC-filled PPS as the intermediate layer, and the PTFEblended PPS as the top layer. The other was comprised of the $\mathrm{Zn.Ph}$ primer and the boehmite-filled PPS only. The lined tubes were set by NREL into the test skids at the 
Mammoth geothermal power plant site, and were exposed for two years to flowing geothermal brine at temperature of $160^{\circ} \mathrm{C}$.

Thus, the objective of the present work was to conduct post-test analyses of the two yearlong exposed liners. The physicochemical factors to be analyzed included the chemistry of the scales deposited on the liner's surfaces, the interfacial bond between the liners and the scales, and the chemical composition and state of liner's surfaces. Integrating these data would provide us with the information on the reliability of these liners in protecting the carbon steel heat exchanger tubes against corrosion, oxidation, and scaling in such a harsh, hostile geothermal environment. For comparison with these liners, we used the stainless steel tubes without any liners.

\subsubsection{Experimental}

The AISI 1008 carbon steel heat exchanger tubes, 20-ft.-long, 1-in. outside diam. with 0.048-in.-thick walls, were lined with the coating materials. The reference stainless steel tube with as same dimension as that of the carbon steel tubes was AISI AL-6XN. The “as-received” PPS powder for the slurry coatings, supplied by the Ticona, had a particle size of $<20 \mu \mathrm{m}$ and a high melt flow at temperatures above its melting point of $250^{\circ} \mathrm{C}$. The PTFE powder under the commercial trade name "SST-3H”, supplied by Shammrock Technologies, was used as the anti-oxidant additive to PPS, and had a particle size of $\sim 40 \mu \mathrm{m}$. PTFE-blended PPS powder, with a PPS/PTFE ratio of 90/10 by weight, was prepared in a rotary blender. The nanoscale boehmite filler (Catapal ${ }^{\circledR} 200$ Alumina) was supplied by Sasol North American Inc.The boehmite filler, at 10 \% by weight of the total amount of PPS, was mixed with the PPS powder in a rotary blender. The SiC grits used to enhance the thermal conductivity of the PPS layer were of the mixture of grits consisting of three different sizes, $\sim 142$, $\sim 32$, and $\sim 9 \mu \mathrm{m}$, obtained from Norton Corporation. The industrial grade isopropyl alcohol was used to make the slurries. Table 4 gives the formulations of the three different slurries, SiC-filled PPS, boehmite-filled PPS, and PTFE-blended PPS that were used to line the internal surfaces

of the heat exchanger tubes. Before deposing these coatings on the interior surfaces of the tubes, the tubes' surfaces were covered with $\mathrm{ZnPh}$ primer. The thickness of these liners without the $\mathrm{ZnPh}$ primer (8 to $60 \mu \mathrm{m}$ ) ranged from 300 to $330 \mu \mathrm{m}$. 
Table 4. Formulation of slurry systems

\begin{tabular}{|c|c|c|c|c|c|}
\hline \multirow{2}{*}{ System } & \multicolumn{5}{|c|}{ Composition, wt \% } \\
\cline { 2 - 6 } & PPS & SiC & Boehmite & PTFE & $\begin{array}{c}\text { Isopropyl } \\
\text { alcohol }\end{array}$ \\
\hline SiC/PPS & 36.0 & 19.0 & - & - & 45.0 \\
\hline $\begin{array}{c}\text { Boehmite/P } \\
\text { PS }\end{array}$ & 45.0 & - & 4.5 & - & 50.5 \\
\hline PTFE/PPS & 43.0 & - & - & 4.3 & 52.7 \\
\hline
\end{tabular}

\subsubsection{Results}

\subsection{Scale Deposition}

Figure 26 shows the SEM microphotograph of the scale deposited on the surfaces of the PTFE-blended PPS top layer. The image revealed the accumulation of flake-like scales covering the entire surface of the top layer. The EDX spectrum of the scale fragments denoted as area “A” had seven elemental distributions, C, O, Na, Si, Cl, Ca, and $\mathrm{Fe}$, as the representative elements of the scales. Among these elements, $\mathrm{Na}$ and $\mathrm{Cl}$ may be attributed to the $\mathrm{NaCl}$ salt, while possible assignments of three other elements, $\mathrm{O}$, $\mathrm{Ca}$, and $\mathrm{Si}$, are the calcium silicate compounds and silica. The Fe-related components of scales may be associated with $\mathrm{FeCl}_{2}$ and Fe oxides. The remaining component of the scales reflects organic contaminants because of the presence of a conspicuous $\mathrm{C}$ signal. The EDX spectrum from the other area marked as site "B" included a pronounced signal from the S element, moderately intense signals of $\mathrm{Si}, \mathrm{Cl}, \mathrm{Ca}$, and $\mathrm{Fe}$, and weaker signals from C, O, F, and Na. The major element S along with the F element appears to be originated from the PTFE-blended PPS coating. All the other elements seem to come from the scales. The EDX is extremely useful for the quantitative analysis of individual elements in a solid surface layer, up to $\sim 1.5 \mu \mathrm{m}$ thick because of the penetration of the $\mathrm{x}$ rays to that depth. Thus, it is possible to assume that the thickness of some scale layers is no more than $1.5 \mu \mathrm{m}$. To the contrary, the EDX spectrum (not shown) of the scales accumulated over the stainless steel tube do not show any typical elements like Cr, Mn, 

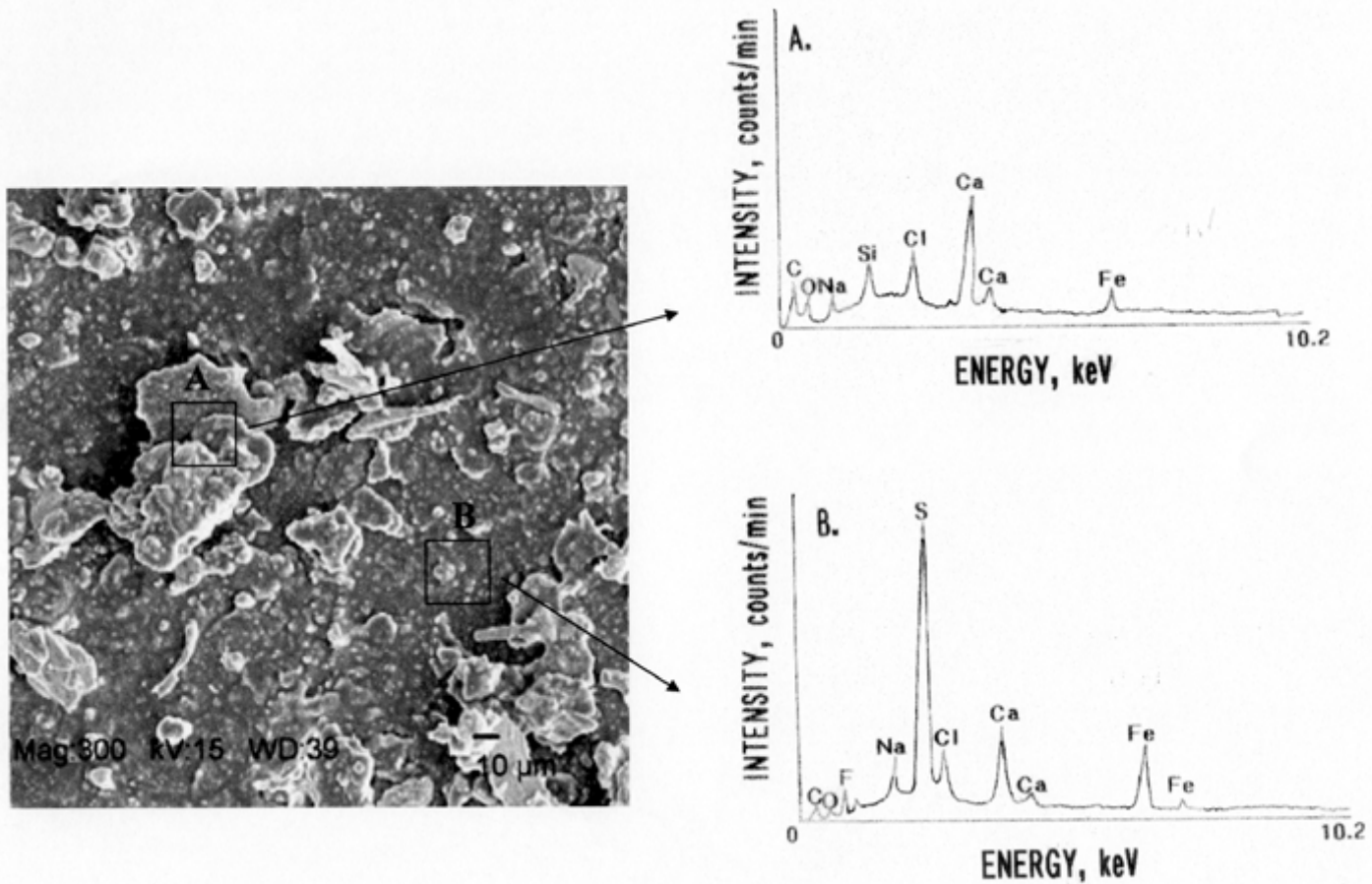

Figure 26. SEM microphotograph and EDX spectra of scale deposited on PTFEblended PPS's surfaces after two-year-long field exposure.

and Ni that are representative of the major chemical components of the stainless steel. This implies that the scale layer deposited on the stainless steel is more than $1.5 \mu \mathrm{m}$ thick. This information strongly suggested that the susceptibility of the stainless steel's surfaces to the scale deposition is much greater than that of the surfaces of the PTFEblended PPS liner.

To support the information on the chemical constituents of the scales, FT-IR analysis (Figure 27) of powdered scales was carried out over the frequency range of 4500 to $450 \mathrm{~cm}^{-1}$. The IR spectrum showed the following absorption bands at 3423 and 1627 $\mathrm{cm}^{-1}$ attributed to $\mathrm{O}-\mathrm{H}$ stretching and bending vibrations of $\mathrm{H}_{2} \mathrm{O}$, respectively, at 2923, 2853, and $1463 \mathrm{~cm}^{-1}$, which can be ascribed to the aliphatic C-H stretching and bending modes of $\mathrm{CH}_{2}$ group, at 1155 and $1095 \mathrm{~cm}^{-1}$, corresponding to the calcium silicate and silica, and at 668 and $602 \mathrm{~cm}^{-1}$, revealing the inorganic Cl-related salts. From the presence of $\mathrm{H}_{2} \mathrm{O}$ molecules, the calcium silicate might be defined as hydrated calcium silicate compounds. 


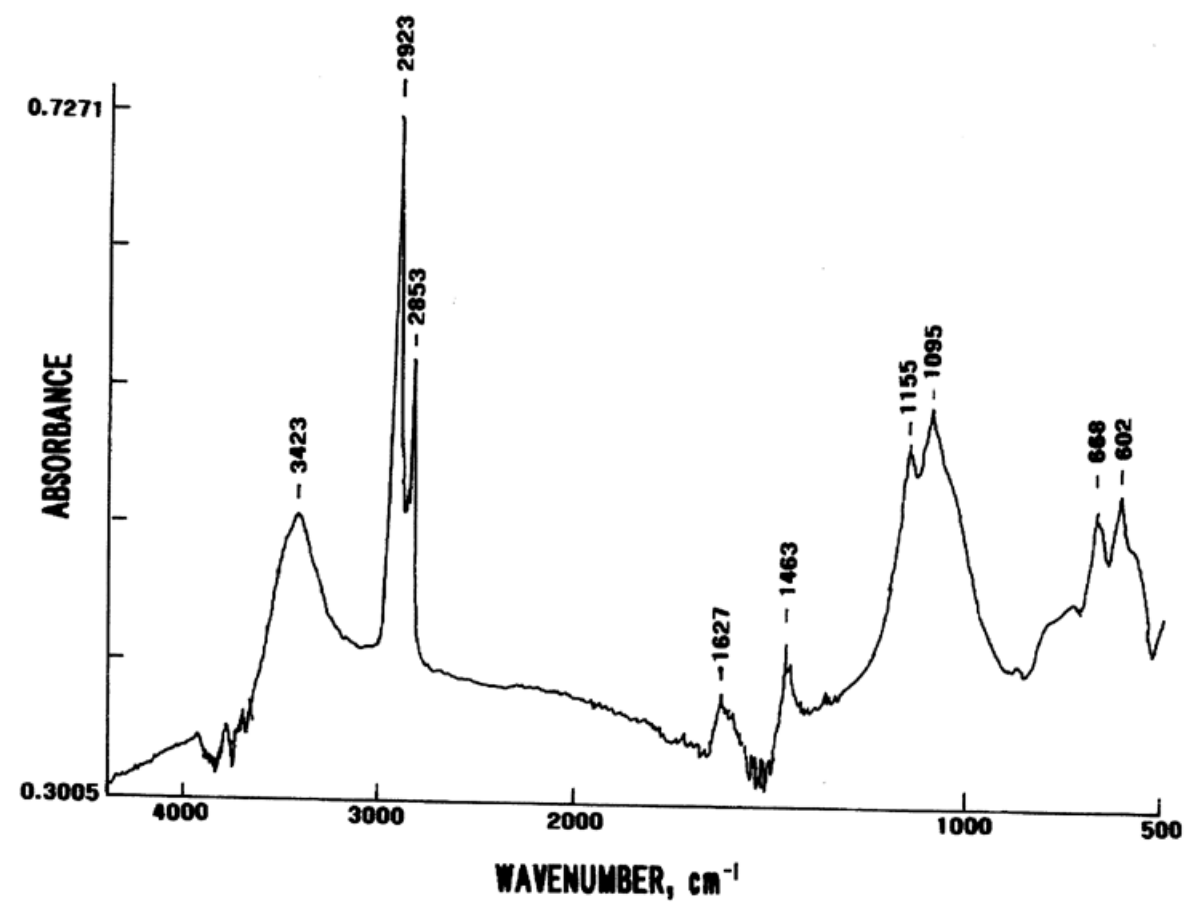

Figure 27. FT-IR spectrum of scale collected from the exposed liner's surfaces.

Next, out attention was focused on assessing how well the scale adheres to the tube's surfaces. We adapted the technique for hydroblasing to obtain this information. Thus, the extent of the adherence of the scales to the surfaces of liners and the stainless steel tube was estimated by determining the hydropressure needed for scouring all scales from the internal surfaces of the 20-ft.-long tubes. The results showed that the scales deposited on both the PTFE-blended PPS and boehmite-filled PPS surfaces were easily removed by relatively low hydropressure, ranging from 10.3 to $13.8 \mathrm{MPa}$. By contrast, hydropressure of as high as 55.1 MPa was required to dislodge the scales from stainless steel, clearly verifying that the scales adhere to the bare stainless steel much more firmly than they do to the liners. Furthermore, despite using such a high hydropressure, we recognized from a visual inspection that some scales still remained on the stainless steel surfaces. To visualize insight into the microstructure of the remaining scales, a cross- sectional area of the tube was explored by SEM (Figure 28). As seen, the scale layer had a thickness of $5 \mu \mathrm{m}$ and was comprised of two different layers marked as "A" and " $\mathrm{B}$ ". The SEM image 


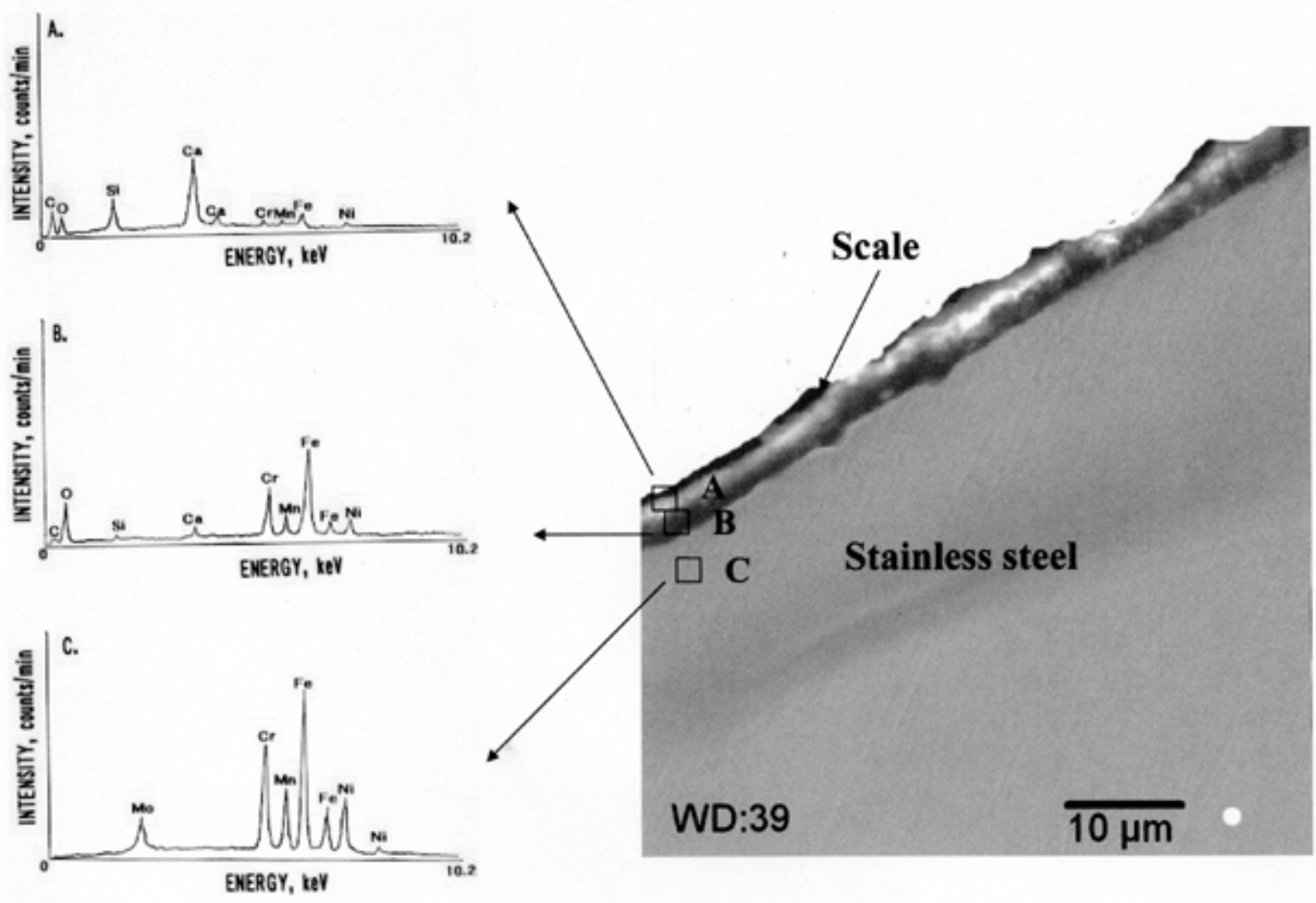

Figure 28. SEM image coupled with EDX analysis for cross-sectional area at critical interfacial region between stainless steel and scale after hydroblasting.

revealed morphological features representing a strong bond between these two layers. The EDX spectrum of the top layer in the scale gave four strong signals from $\mathrm{C}, \mathrm{O}, \mathrm{Si}$, and $\mathrm{Ca}$, as the principal elements. The contributors to these elements are calcium silicate hydrate, silica, and organic contaminants. There were no $\mathrm{Na}$ and $\mathrm{Cl}$ elements from salt, inferring that the salt compounds being present in the original scales were washed away by hydroblasting. The EDX data of the bottom layer was characterized by three prominent peaks related to the $\mathrm{O}, \mathrm{Cr}$, and Fe elements, moderately intense $\mathrm{Ca}, \mathrm{Mn}$, and Ni peaks, and weak C and Si signals. As shown by EDX, stainless steel has five major elements, Mo, Cr, Mn, Fe, and Ni. Hence, the source of these four elements that were detected in the bottom layer appears to be the stainless steel. Relating these metal elements to the marked signal of $\mathrm{O}$ element, we assumed that these metals occupying the outermost surface sites of the steel were oxidized during its exposure. If this interpretation is valid, these metal oxide layers that formed over the steel surfaces have some affinity for calcium silicate and silica scales, thereby forming a strong interfacial 
bond between the metal oxide and the scales. This is a main reason why scales deposited on the stainless steel were very difficult to scour away.

In contrast, the scales over the PTFE-blended PPS surfaces were flaked off readily by low-pressure hydroblasing. Compared with the SEM image (Figure 26) from a surface fouled by scale deposits, a dramatic change can be seen after hydroblasting (Figure 29); namely, there are no remnants of any scales. In fact, the EDX analysis did not detect any scale-related elements, such as $\mathrm{Ca}, \mathrm{Si}, \mathrm{Na}, \mathrm{Cl}$, and $\mathrm{Fe}$, demonstrating that
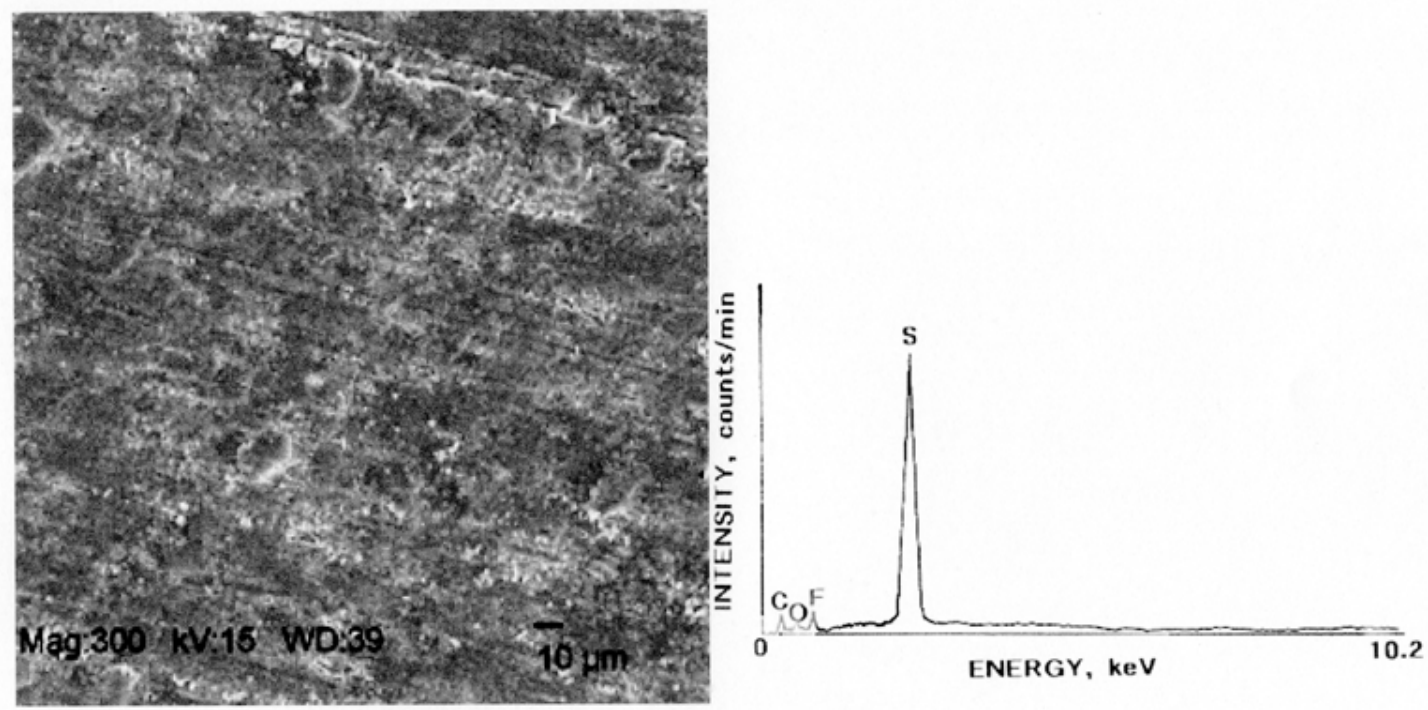

Figure 29. SEM-EDX analysis of PTFE-blended PPS's surface after hydroblasting.

the surfaces of PTFE-blended PPS liner are insensitive to reactions with the scales. This means that the extent of the interfacial bonding force between the liner and the scale is minimal, if any. The SEM image (not shown) of the surfaces of the boehmite-filled PPS liner after hydroblasting showed no significant differences to that of unexposed surfaces. Also, SEM exploration revealed no signs of any surface damages brought about by hydroblasting. However, although the signal intensity was very weak, the EDX (not shown) indicated the presence of $\mathrm{Si}$, which is one of the scale-related elements. Relating this $\mathrm{Si}$ signal to the $\mathrm{O}$ signal that also was detected, a possible interpretation is that some silica seems to remain on the hydroblasted liner's surfaces. 


\subsection{Liner's Surfaces}

To understand why the PPS liner's surfaces without the PTFE become susceptible to the deposition of silica scales during exposure, we investigated the changes in chemical composition of the hydroblasted liner's surfaces after exposure, compared with that of the unexposed ones. The surfaces for the PTFE-blended PPS and boehmite-filled PPS liners before and after exposure were analyzed using XPS. All XPS measurements were made at an electron take-off angle of $40^{\circ}$, which corresponds to an electronpenetration depth of $\sim 5 \mathrm{~nm}$, reflecting the detection of atomic fraction and chemical states present in a superficial layer with a thickness of $\sim 5 \mathrm{~nm}$. Table 5 gives the XPS atomic fractions of all chemical elements detected on the surfaces of liners. The quantitative data for the respective chemical elements were estimated by comparing the XPS $\mathrm{Si}_{2 \mathrm{p}}, \mathrm{S}_{2 \mathrm{p}}, \mathrm{C}_{1 \mathrm{~s}}, \mathrm{O}_{1 \mathrm{~s}}$, and $\mathrm{F}_{1 \mathrm{~s}}$ core-level areas, which were then converted into atomic concentrations. Before exposure, the atomic fraction of PTFE-blended PPS's surfaces

Table 5. Atomic composition of the surfaces of liners before and after two yearlong field exposure test

\begin{tabular}{|c|c|c|c|c|c|c|c|}
\hline \multirow[t]{2}{*}{ Liner } & \multirow{2}{*}{$\begin{array}{c}\text { Field } \\
\text { test }\end{array}$} & \multicolumn{5}{|c|}{ Atomic fractions, $\%$} & \multirow{2}{*}{$\begin{array}{c}\text { Atomic } \\
\text { ratio, } \\
\text { O/C }\end{array}$} \\
\hline & & Si & $\mathbf{S}$ & $\mathbf{C}$ & $\mathbf{O}$ & $\mathbf{F}$ & \\
\hline $\begin{array}{c}\text { PTFE- } \\
\text { blended } \\
\text { PPS }\end{array}$ & Before & 0.0 & 2.1 & 48.0 & 2.6 & 47.3 & 0.054 \\
\hline $\begin{array}{l}\text { PTFE- } \\
\text { blended } \\
\text { PPS }\end{array}$ & After & 0.0 & 1.3 & 48.8 & 3.1 & 46.8 & 0.064 \\
\hline $\begin{array}{c}\text { Boehmite } \\
\text {-filled } \\
\text { PPS }\end{array}$ & Before & 0.0 & 13.1 & 81.6 & 5.3 & 0.0 & 0.065 \\
\hline $\begin{array}{c}\text { Boehmite } \\
\text {-filled } \\
\text { PPS }\end{array}$ & After & 0.4 & 9.7 & 76.9 & 12.8 & 0.0 & 0.167 \\
\hline
\end{tabular}

consisted of two dominant atoms, $48.0 \% \mathrm{C}$ and $47.3 \% \mathrm{~F}$, and two minor ones, $2.6 \% \mathrm{O}$ and $2.1 \% \mathrm{~S}$. The source of $\mathrm{F}$ and $\mathrm{C}$ atoms is the PTFE. Thus, we believe that the superficial layer (no more than $5 \mathrm{~nm}$ thick) was made up almost exclusively of PTFE. 
This phenomenon was due to the typical separation of PTFE from PPS and its migration upwards to the top surface of the liner. Consequently, PTFE occupied the liner's outermost surface site. By comparison, no conspicuous difference in the atomic fraction was obtained from the exposed liner's surfaces. Although some oxygen was incorporated in the superficial layer, its amount was negligible.

The boehmite-filled PPS liner's surfaces were comprised of $13.1 \% \mathrm{~S}, 81.6 \% \mathrm{C}$, and $5.3 \%$ O. No boehmite-related $\mathrm{Al}$ atom was detected, reflecting the coverage of the entire surfaces of all boehmite crystals by a PPS layer, at least $5.0 \mathrm{~nm}$ thick. For the surfaces of the exposed liner, our attention was centered on incorporating a substantial amount of oxygen into the top surface layer. The atomic ratio of $\mathrm{O} / \mathrm{C}$ of the exposed liner rose 2.6 fold to 0.167 compared to the unexposed one. This can be taken as evidence that the PPS's surfaces underwent some degree of hot brine-catalyzed oxidation. The data also indicated that $0.4 \% \mathrm{Si}$ atom was incorporated into the superficial layer. Thus, the oxidation products formed at outermost surface site seem to have an undesirable function similar to that of the oxide compounds accruing on stainless steel's surfaces; namely, they are sensitive to silica deposition. From the above information, PTFE as the antioxidant additive of PPS appears to play an essential role in reducing the rate of the scale deposition and in creating an inert surface to reactions with the scale in the two years exposure periods.

\subsubsection{Field Test at Puna Power Plant}

Our research team including BNL, NREL, and Thermochem Corp. completed a four-week field performance test at the Puna Power Plant, Hawaii, operating at temperature of $\sim 200^{\circ} \mathrm{C}$, of the thermally conductive micorscale carbon finer-reinforced PPS composite-lined HX tubes (0.5-in.-inner diameter by 20-ft.-long) fabricated by BNL. In this test, stainless steel (SS) tube was used as the control. Since most of our field tests thus far were conducted under brine at around $160^{\circ} \mathrm{C}$, this field test was the first opportunity for validating the integrity of PPS-based lining systems at a higher brine temperature of $200^{\circ} \mathrm{C}$.

In this post-test analysis, our focus was centered on analyzing the PPS composite liners deposited on Vaporizer tubes because the test condition of the Vaporizer tube 
$\left(196^{\circ} \mathrm{C}\right.$ inlet and $115^{\circ} \mathrm{C}$ outlet at $\left.\mathrm{pH} 4.5\right)$ was much severer than that of PPS-lined Preheater tube $\left(115^{\circ} \mathrm{C}\right.$ inlet and $77^{\circ} \mathrm{C}$ outlet at $\left.\mathrm{pH} 4.5\right)$.

\subsubsection{Results}

The scale accumulated over the PPS Vaporizer liner's surface at inlet was identified as silica (Figure 30), and its thickness was roughly $88 \mu \mathrm{m}$ (Figure 31). The scale was more likely to be deposited on the inlet liner, rather than on the outlet liner. SEM image (Figure 31) clearly showed that in some areas, the PPS liner poorly adhered to the underlying steel. This poor adherence was due primary to an inadequate deposition of $\mathrm{ZnPh}$ primer on the underlying steel's surface. In fact, an undesirable deposition of ZnPh on the tube's surface can be seen in the SEM image and EDX spectrum indicating a very weak signal of the $\mathrm{Zn}$ element for the tube sample beneath the delaminated liner. An ideal ZnPh layer deposited uniformly over the entire surfaces of tubes must have a thickness of at least $10 \mu \mathrm{m}$. The crystalline $\mathrm{ZnPh}$ primer layer having a very rough surface played an important role in stabilizing the interfacial bond at the $\mathrm{PPS} / \mathrm{ZnPh} / \mathrm{steel}$ joint. Since the longitudinal coefficient of thermal expansion of the PPS liner was more than five fold higher than that of the carbon steel tube $\left(1.3 \times 10^{-5} \mathrm{~mm} / \mathrm{mm}^{\circ} \mathrm{C}\right.$ for steel vs. $6.8 \times 10^{-5} \mathrm{~mm} / \mathrm{mm}^{\circ} \mathrm{C}$ for PPS liner at $300^{\circ} \mathrm{C}$ ), a strong bonding between the PPS and $\mathrm{ZnPh}$ was required to avoid a different linear thermal expansion-caused segregation of the PPS liner from the smooth tube's surface during the fabrication process of liner. Fortunately, the PPS had a great affinity with $\mathrm{ZnPh}$ due to two interfacial bonding mechanisms: One was a chemical bond; the other was a mechanical anchoring of the PPS polymer, which resulted from the penetration of the molten polymer into the open surface microstructure of the $\mathrm{ZnPh}$ layers at $300^{\circ} \mathrm{C}$. Correspondingly, most of liners excepting for delaminated and blistering areas displayed a satisfying interfacial bond (Figure 32). In preparing the $\mathrm{ZnPh}$ for those test tubes with a diameter of less than a half inch, we adapted the standard $\mathrm{ZnPh}$ conversion solution that is still commonly used for one-inch diameter tubes. Although there was no experimental evidence as to why this solution was no longer as effective as applied to the one-inch tube, the modification of this formulation might be needed for smaller diameter tubes. 

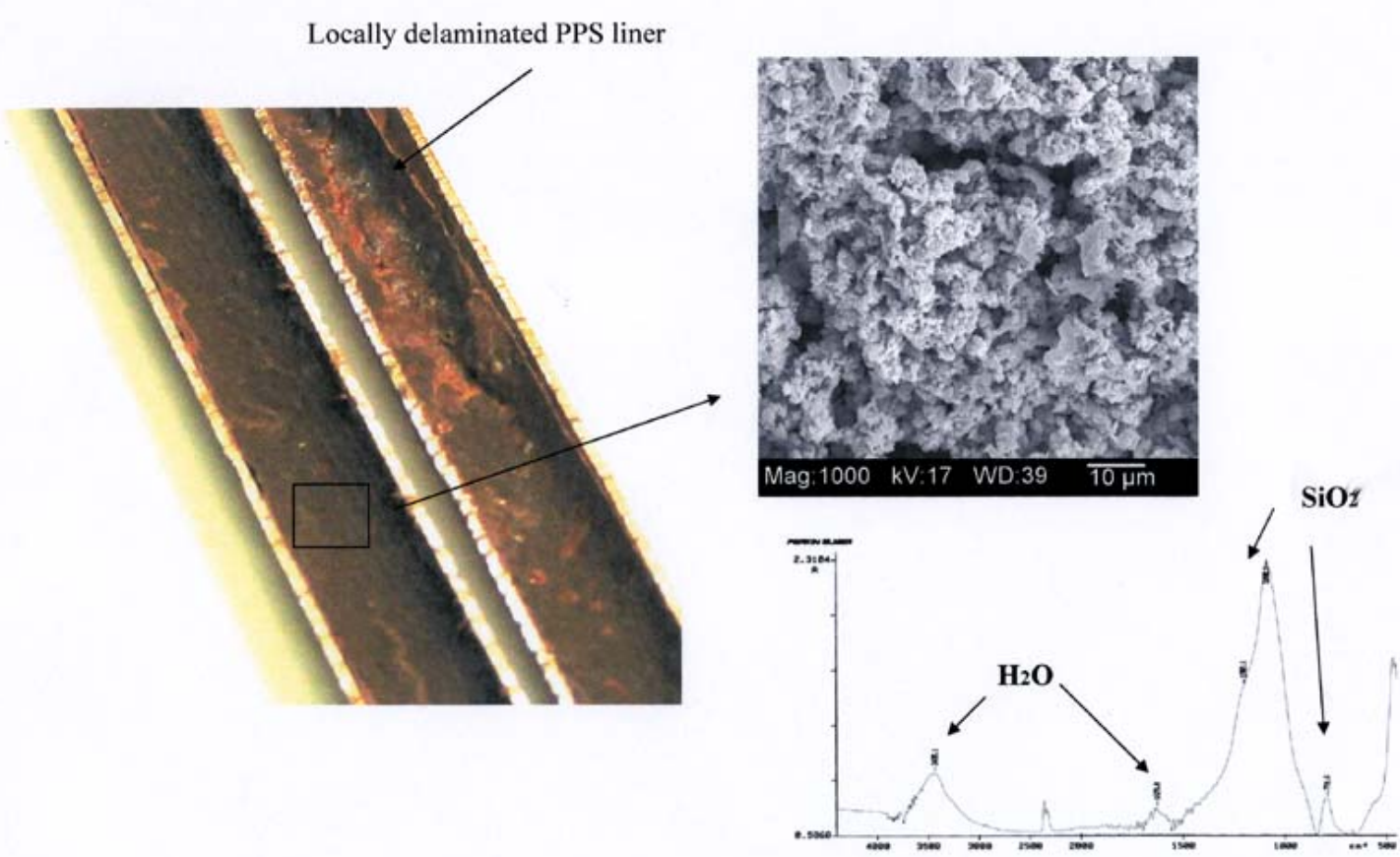

Figure 30. SEM image and FT-IR spectrum of scales deposited on the liner's surface as well as visual observation of locally delaminated liner after exposure at inlet.

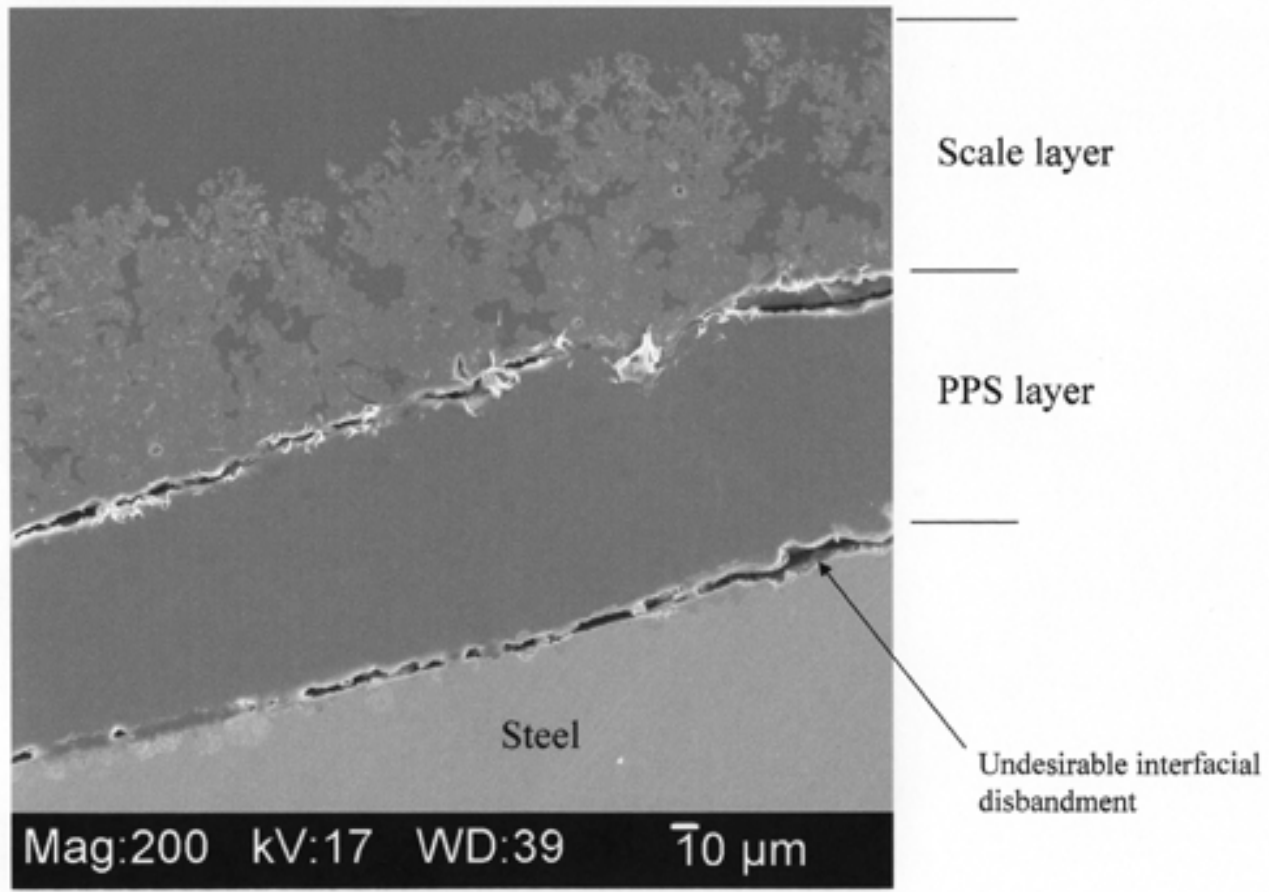

Figure 31. Cross-sectional profile of PPS-lined tube after exposure at inlet. 


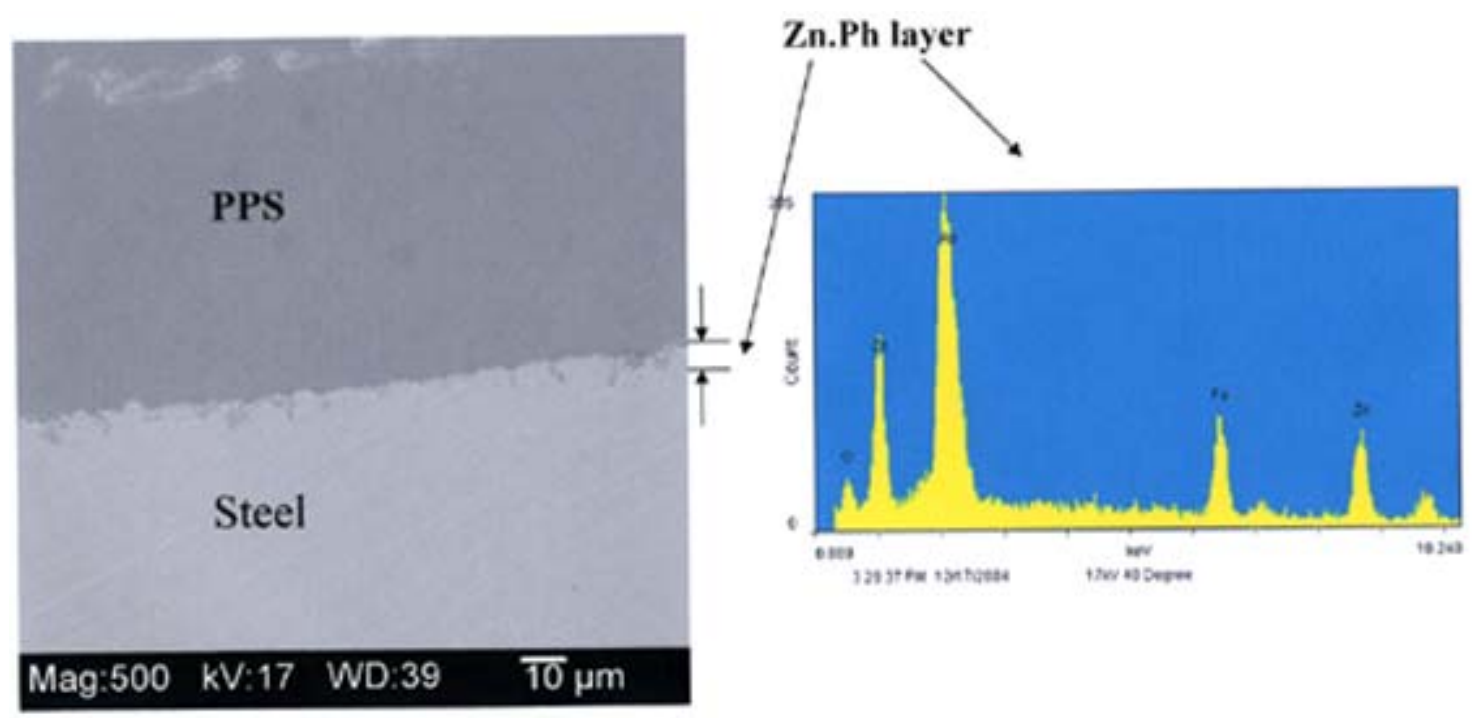

Figure 32. Ideal interfacial bond between PPS and ZnPh primer observed in the lined tube after exposure at inlet.
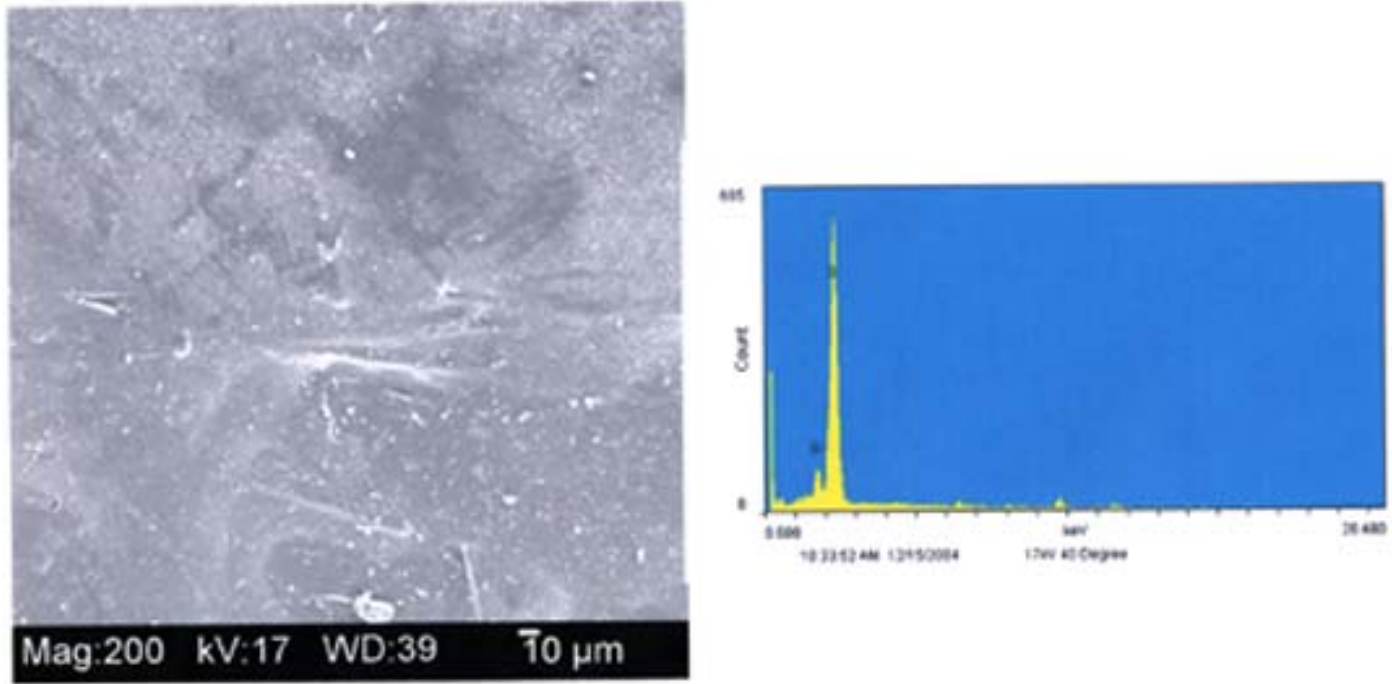

Figure 33. Surface image of liner after removal of scale by rinsing with water. 


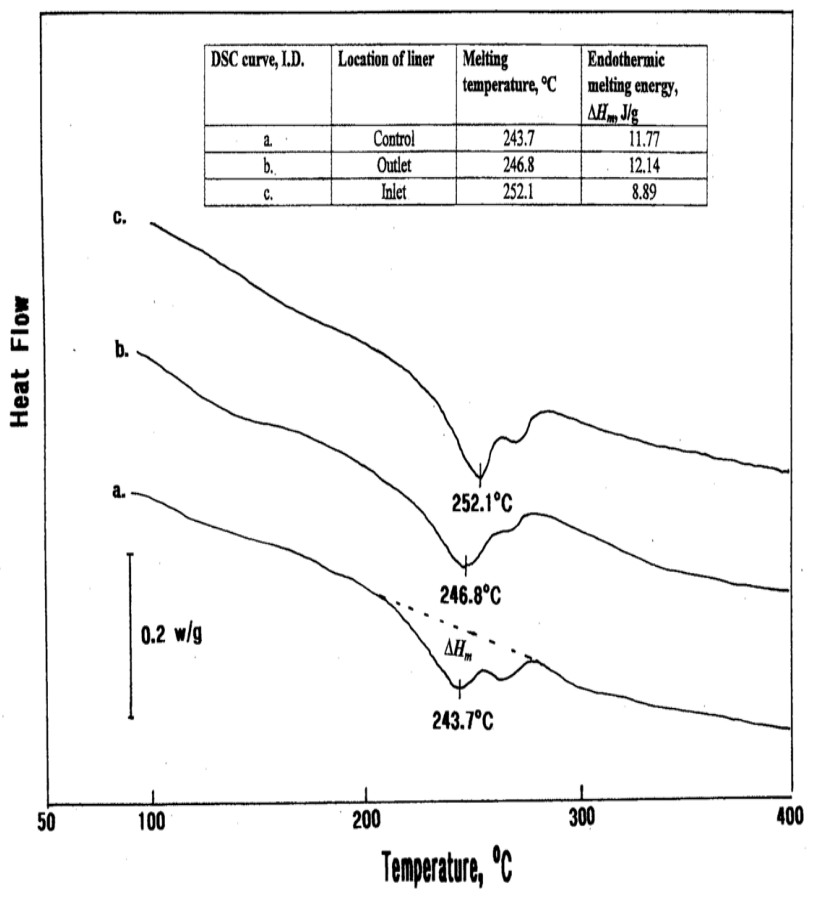

Figure 34. Melting point and melting energy of PPS liners before exposure (a), and after exposure at outlet (b) and at inlet (c) by differential scanning calorimeter (DCS).

The analytical work next sifted to investigating the integrity and quality of PPS liners after exposure. Figure 33 shows the surface texture of PPS inlet liner's surface after removal of scale, revealing no signs of the generation of any fissures brought about by its thermal degradation. In addition, the combined analyses of DSC (Figure 34) and TGA (Figure 35) for the unexposed, and outlet- and inlet-exposed liners strongly demonstrated that the integrity of exposed PPS liners remained in effect because of no significant changes in thermal properties of exposed liners compared with those of unexposed one. 


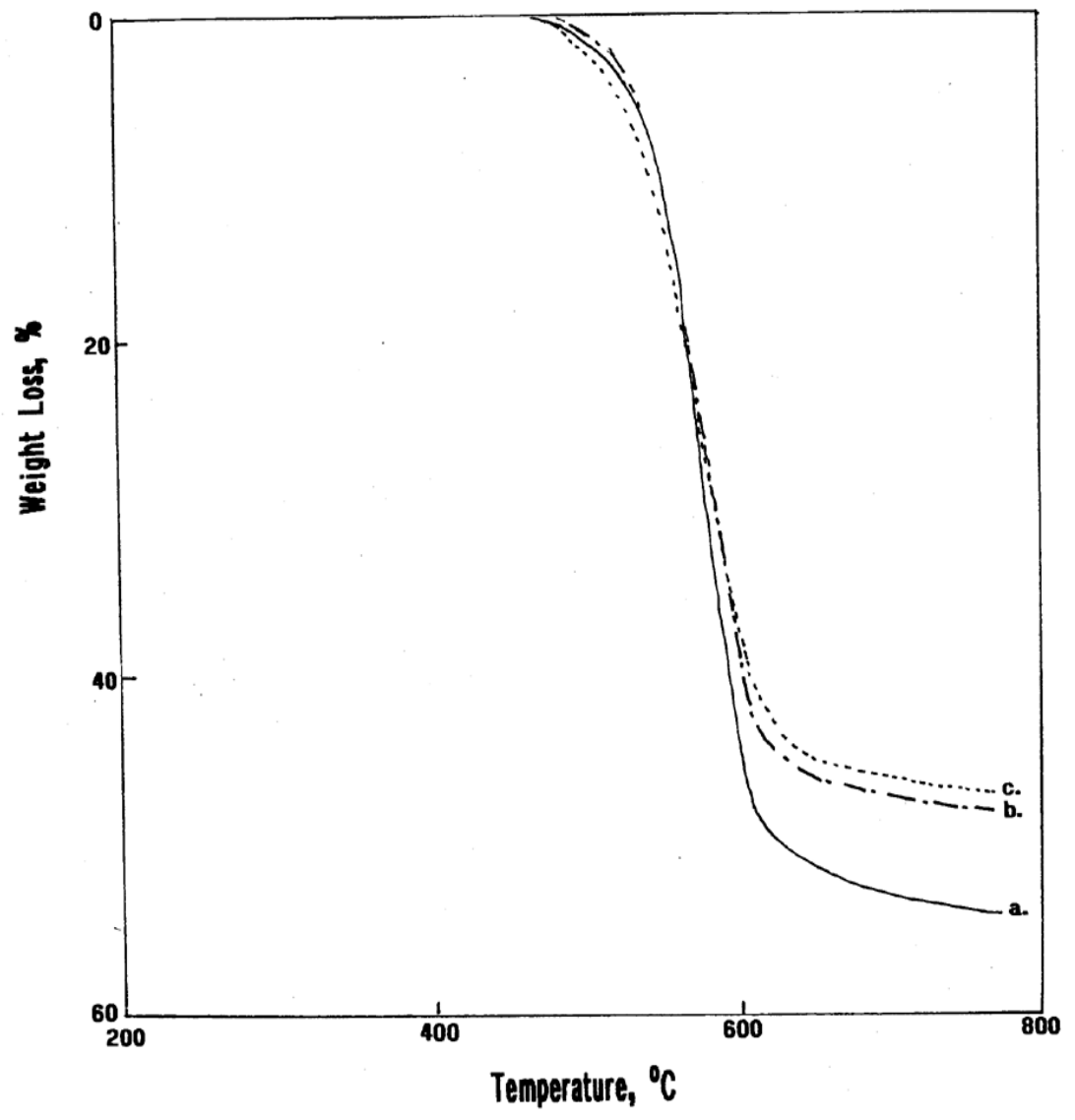

Figure 35. Onset of thermal decomposition for unexposed (a), and outlet (b)-and inlet (c)-exposed liners by thermogravimetric analyzer (TGA).

One issue to be addressed involves the characteristic of scale deposited on the stainless steel (SS). Figure 36 shows the SEM-EDX close-examination of interfacial areas between the scale and SS. As seen, the image represented two distinctive scale layers: One was a densified scale layer about $\sim 5 \mu \mathrm{m}$ thick adhering to the SS's surface; the other referred to the same silica scale layer as that observed from the liner's surface. The EDX for the former scale layer revealed that this scale was attributed to silicatebased scales containing two additional elements, $\mathrm{K}$ and $\mathrm{Ca}$, especially for the calcium silicate scale. The detected $\mathrm{Al}$ element arises from the $\mathrm{Al}_{2} \mathrm{O}_{3}$ polishing powder used to prepare a smooth cross-sectional sample. The ordinal silica scale layer could be easily 


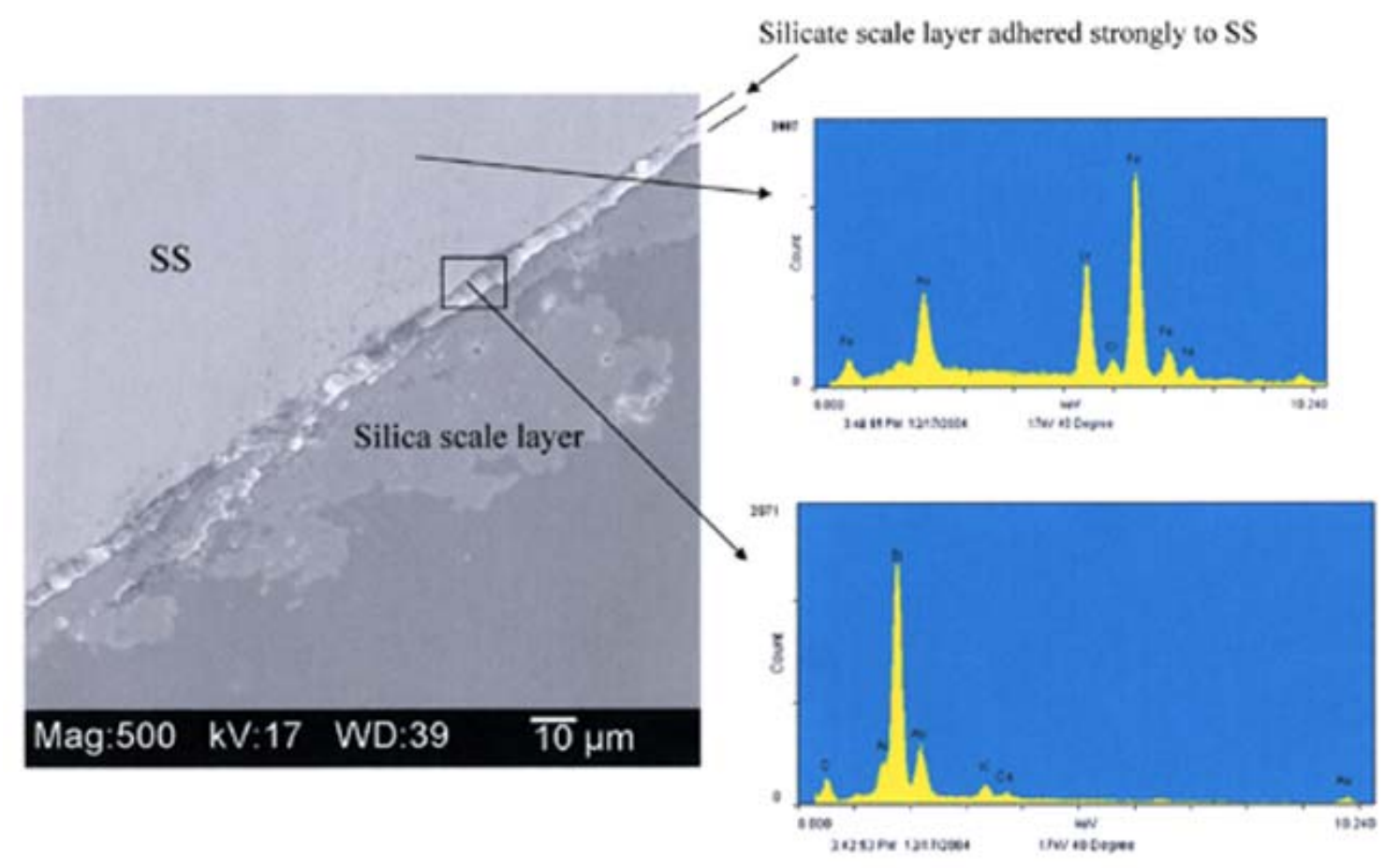

Figure 36. SEM image of two distinctive scale layers deposited on the SS's surface after exposure: Silicate scale layer adhered to SS and ordinal silica scale layer accumulated over silicate scale.

dislodged from the SS by hydroblasting at a lower pressure, but not a silicate scale layer as the irremovable scale adhered strongly to metals. We assumed that this silicate scale would grow as a function of an exposure time. In contrast, the PPS's surface was chemically and mechanically inert with scale. There was no development of the additional scale layer attributed to silicate compounds at the interfaces between the scale and PPS.

Based upon the information described above, the following statement can be drawn as the conclusion: The generation of local delamination and blisters of PPS liners incurred at inlet $\left(196^{\circ} \mathrm{C}\right)$ was more likely to be associated with the poorly deposited Zn.Ph primer layer on the tube's surface, rather than the hydrothermal degradation of PPS liners. Thus, the chemical modification of the standard $\mathrm{ZnPh}$ solution will be required to deposit a rough crystalline $\mathrm{ZnPh}$ layer of at least $10 \mu \mathrm{m}$ thick for tubes with a diameter of $<0.5$ in. 


\subsection{Conclusions}

The following general conclusions can be drawn from the results of in-house and field works for thermally conductive lining materials of carbon steel heat exchanger (HX) tubes.

1. The interfacial bond structure for the polyphenylenesulfide (PPS)/ zinc phosphate $(\mathrm{ZnPh})$ primed steel joint systems had both mechanical interlocking and chemical bonds. For the former bond structure, metallographic observations suggested that the melted PPS thermoplastic penetrates into open-surface microstructure and microfissures in the crystalline $\mathrm{ZnPh}$ layers, thereby contributing to making a good bond at the PPS-to-ZnPh interfaces, by the mechanical anchoring PPS polymer. The latter bond structure was characterized by representative of the formation of zinc sulfide ( $\mathrm{ZnS}$ ) reaction product yielded by chemical interactions between PPS and $\mathrm{Z}$ in the $\mathrm{ZnPh}$ primer layer during exposure in a wet, harsh geothermal environment. The combined bond structures from mechanical and chemical linkages significantly enhanced the extent of the primer-to-PPS adhesive force. The ZnS reaction product at interfaces played a major role in inhibiting the alkali-induced delamination of PPS film from the steels that was caused by cathodic reaction, $\mathrm{H}_{2} \mathrm{O}+1 / 2 \mathrm{O}_{2}+2 \mathrm{e}^{-} \rightarrow 2 \mathrm{OH}^{-}$, occurring at corrosion sites in the steel, because of its insolubility in the alkali solution at $\mathrm{pH} 13$. Thus, this reaction product prevented the alkali dissolution of the intermediate layers, thereby reducing significantly the rate of cathodic delamination of PPS film from the steel.

2. Compared with the chopped carbon fibers of $7.5 \mu \mathrm{m}$ diameter and $\sim 3 \mathrm{~mm}$ long, the major advantage of the shorter milled carbon microfibers (100-200 $\mu \mathrm{m})$ with the same diameter was that it allowed us to incorporate substantially more of the fibers into the PPS coatings. This advantage enhanced the beneficial properties of the coatings; for instant, the thermal conductivity of the PPS coatings raised 2.6 fold to $3.7 \mathrm{~kJ} / \mathrm{hr} . \mathrm{m} .{ }^{\circ} \mathrm{C}$ by incorporating $5 \mathrm{wt} \%$ fiber. Furthermore, $3 \mathrm{wt} \%$ fiberreinforced PPS coating films displayed a great tensile strength and elongation of $40.8 \mathrm{MPa}$ and $5.8 \%$, respectively, corresponding to the improvement of 5.2 times 
and 2.6 times over those of the non-reinforced coatings. However, increasing the amount of fibers, beyond $3 \mathrm{wt} \%$, caused a gradual decline in these mechanical properties. Although we have no experimental evidence, we assumed that such mechanical retrogression is due to the deficient coverage of the fibers' surfaces with PPS caused by incorporating an excessive amount of fibers into the matrix, reflecting the development of low interfacial shear bond strength at the fiber/PPS joint. Although the thermal conductivity of the PPS coatings depended preliminary on their fiber content, the most effective amount of fiber in improving mechanical behaviors was $3 \mathrm{wt} \%$.

3. The $3 \mathrm{wt} \%$ carbon fiber-reinforced PPS composite coating reduced the rate of uptake of corrosive electrolytes by the coating, compared with that of the PPS without carbon fiber, ensuring a better performance in protecting the underlying steel against corrosion in a $200^{\circ} \mathrm{C}$ brine environment. However, incorporating an excessive amount of fiber into PPS impaired the efficacy of the coatings in mitigating the corrosion of steels because of the development of an undesirable porous microstructure due to the air-trapping behavior of the fibers.

4. Ferric oxide $\left(\mathrm{Fe}_{2} \mathrm{O}_{3}\right)$ occupying the outermost surface site of carbon steel had a strong affinity for silica. In fact, when steel panels were immersed in sodium metasilicate-dissociated brine at $200^{\circ} \mathrm{C}$, silica aggressively precipitated on the steel surfaces after immersion for only 24 hours. Further, the silica scales strongly adhered to the steel surfaces, and it was very difficult to scour them off. Although there was no deposition of micro-sized silica over the PPS coating surfaces after 7-day immersion, we detected a negligible amount of silica in the superficial layer ( $~ 5$ nm thickness) of the coatings, suggesting that the surfaces of immersed PPS become sensitive to silica deposits. The creation of such sensitivity was the functional sulfur oxide derivatives yielded by a hot brine-induced oxidation of PPS surfaces. Thus, the formation of an oxide surface layer on either PPS or carbon steel can be taken as one of the critical factors affecting the initiation of silica deposits. In trying to deal with this problem, blending 
polytetrafluoroethylene (PTFE) as the antioxidant into the PPS not only offered improved oxidation resistance of the coating surfaces, but also served in creating the hydrophobic surfaces. The main reason for conferring such improvements was due to the phase segregation of PTFE from the PPS, whereby segregated PTFE formed the outermost surface layer of coating. Therefore, PTFE antioxidantblended PPS coatings had a high potential for use as anti-silica fouling barriers on the carbon steel heat exchanger tubes in geothermal environments containing silica-rich brine.

5. In trying to confer resistance of PPS coatings to blasting wear, nanoscale boehmite fillers with a crystalline size of $\sim 30 \mathrm{~nm}$ were incorporated into PPS coatings. The rate of blasting wear of the unfilled coating was reduced six-fold by adding $15 \mathrm{wt} \%$ boehmite.

6. Calcium alumina (CA) filler was identified as a material with high potential material for sealing and repairing cracks of $\sim 5.3 \mu \mathrm{m}$ wide and $\sim 8$ to $\sim 17 \mu \mathrm{m}$ deep generated in the superficial layer of coatings exposed in the $200^{\circ} \mathrm{CO}_{2}$-laden brine environments. The mechanisms of healing and repairing may be as follows. When the CA fillers disclosed in the grooves come in contact with hot $\mathrm{CO}_{2}$-laden brine, the carbonation and decalcification-hydration reactions of two reactants in the $\mathrm{CA}, \mathrm{CaO} \cdot \mathrm{Al}_{2} \mathrm{O}_{3}$ and $\mathrm{CaO} .2 \mathrm{Al}_{2} \mathrm{O}_{3}$, lead to the formation of two crystalline reaction products, boehmite and calcite, within 2 hours. In particular, the boehmite phase represented by block-like crystals rapidly grows and seals the open grooves. A microscopic agglomeration of well-developed boehmite crystals was observed in the grooves of the damaged coatings after only 24 hours exposure. The outstanding performance of boehmite crystals in reconstituting and restoring the blemished coatings was validated from measurements of the conductivity of corrosive ions; the pore resistance, $\mathrm{Rp}$, value of the failed and blemished coatings rose nearly two orders of magnitude to $3 \times 10^{8} \mathrm{ohm}-\mathrm{cm}^{2}$ after 24 hours exposure. This finding clearly proved that the function of the coatings in protecting the underlying steel against corrosion was restored within that time. No 
change in this value was recorded from coatings further exposed up to 20 days, suggesting that even then, the boehmite crystals packing the grooves do no allow corrosive ions to permeate through them. Although the long-term exposures are needed to validate that the healed coatings continue to protect the underlying steel, we believe that this self-healing and -repairing technology based upon the rapid growth of boehmite crystals is applicable to coatings exposed to hydrothermal environments. We found that about $5 \%$ of the total weight of PPS was the effective amount of CA content in reconstituting and restoring the anticorrosive function of failed coatings.

7. In trying to protect carbon steel heat exchanger (HX) tubes against corrosion and fouling by calcium silicate hydrate and silica scaling in geothermal binary-cycle Mammoth power plants operating at a brine temperature of $160^{\circ} \mathrm{C}$, their internal surfaces were lined with two thermally conductive high-temperature performance material systems. The lined 20-ft.-long tubes ( 1.0 in. outside diameter) then were exposed for two years at the Mammoth power plant site to ensure that these lining systems satisfactory solved these problems facing the geothermal energy industry. One of in these material systems was comprised of three different lining layers, $\mathrm{Zn}$.Ph as the primer, silicon carbide (SiC) thermal conducter-filled polyphenylenesulfide (PPS) as the intermediate layer, and polytetrafluoroethylene (PTFE)-blended PPS as the top surface layer. The other consisted of the $\mathrm{Zn} . \mathrm{Ph}$ primer and nanoscale boehmite-filled PPS layer. AISA AL-6XN stainless steel tubes also were used as the reference bear steel.

The unlined stainless steel HX tube is well protected against corrosion due to the formation of passive $\mathrm{Cr}, \mathrm{Fe}$, and Mn oxide layers at its outermost surface sites. However, these oxide layers were very receptive to the deposition of calcium silicate hydrate and silica scales, which developed a strong adherence to the tubes. This strong bond is reflected in the requirement for high-pressure hydroblasting of 55.1 MPa to scour them off from the tube's surfaces. But, even then, many scales forming a $~ 2.0 \mu \mathrm{m}$ thick layer still remained on the oxide layer after hydroblasting. 
By contrast, the surfaces of a PPS top layer modified with PTFE as an anti-oxidant additive significantly retarded the hydrothermal oxidation of the liner. Such an anti-oxidant surface not only minimized the rate of the scale deposition, but also made it inert to reactions with the scales. Thus, all the scales deposited on the liner's surfaces were easily removed by hydroblasting with only $\sim 18.0 \mathrm{MPa}$ pressure. In addition, the PPS satisfactory withstood a $160^{\circ} \mathrm{C}$ brine temperature and displayed a great resistance to the permeation of brine through the liner, expressing an outstanding performance in protecting the tubes against corrosion.

The boehmite-filled PPS liner's surfaces without the PTFE suffered some degree of the oxidation, causing the remnant of few silica scales on the hydroblasted liner's surfaces. However, there was no internal delamination of the PPS liner from the $\mathrm{ZnPh}$ primer, nor were any blisters generated in the critical interfacial boundary region between the PPS and the $\mathrm{ZnPh}$ primer. Furthermore, brine-related elements were not detected in a superficial layer of $\sim 5 \mu \mathrm{m}$ thick, strongly demonstrating that although the boehmite filler was incorporated, this liner adequately prevented corrosion of the tubes.

8. The chemical state of scale deposited on the surfaces of thermally conductive microscale carbon fiber-reinforced PPS composite liners and stainless steel (SS) tube at Puna Power Station operating at $\sim 200^{\circ} \mathrm{C}$ was identified as silica. This silica scale strongly adhered to the oxide layer occupying the outermost surface side of SS tube, causing an accumulation of $\sim 88 \mu \mathrm{m}$ thickness after only four weeks exposure. In contrast, the surfaces of composite liners were much more inert to scale deposition. Although the exposure time was only four weeks, this composite liners withstood hot brine at $\sim 200^{\circ} \mathrm{C}$. Thus, we believed that the PPS composite material had a high potential as an anti-fouling and $200^{\circ} \mathrm{C}$ hydrothermal stable liner. However, the generation of local delamination and blisters of PPS liners incurred at inlet was more likely to be associated with the poorly deposited $\mathrm{ZnPh}$ primer layer on the tube's surface, rather than the hydrothermal degradation of PPS liners. Thus, the chemical modification of the 
standard $\mathrm{ZnPh}$ solution will be required to deposit a rough crystalline $\mathrm{ZnPh}$ layer of at least $10 \mu \mathrm{m}$ thickness for tubes with a diameter of $<0.5 \mathrm{in}$.

\subsection{Technology Transfer}

Curran International Corp., commercialized this PPS-based lining material system under the trade name "CurraLon". The company estimated that the capital costs of the heat exchanger, containing on average 800 tubes, would be strikingly reuduced by $\sim 83$ $\%$ and $\sim 80 \%$, compared to those of titanium- and stainless steel-based HXs, respectively.

\subsection{Awards}

In 2002 and 2003, this high-performance PPS composite lining system was selected for the prestigious "Research and Development (R\&D) 100 Award" and "Federal Laboratory Consortium for Technology Transfer (FLC) Award".

\subsection{Publications}

- Sugama, T. and Gawlik, K., "Nanoscale Boehmite Filler for Corrosion- and Wear-resitant Polyphenylenesulfide Coatings”, Polymers \& Polym. Comp., 12, 153-167 (2004).

- Sugama, T. and Gawlik, K., "Self-reparing Poly(phenylenesulfide) Coatings in Hydrothermal Environments at 200”’, Matter. Letters, 57, 4282-4290 (2003).

- Sugama, T. and Gawlik, K., "Milled Carbon Microfiber-reinforced Poly(phenylenesulfide) Coatings for Abating Corrosion of Carbon Steel”, Polymers \& Polym. Comp., 11, 161-170 (2003).

- Sugama, T. and Gawlik, K., "Anti-silica Fouling Coatings in Geothermal Environments”, Matter. Letters, 57, 666-673 (2002).

- Sugama, T., Elling, D. and Gawlik, K., "Poly(phenylenesulfide)-based Coatings for Carbon Steel Heat Exchanger Tubes in Geothermal Environments”, J. Mater. Sci., 37, 4871-4800 (2002).

- Sugama, T., Kelly, S.S., and Gawlik, K., "Hydrothermal Degradation Study of Phenolic Polymer Coatings by Advanced Analytical Methods”, J. Coatings Tech., $\underline{73}, 65-71$ (2001). 
- Sugama, T. and Gawlik, K., "Carbon Fiber-reinforced Poly(phenylenesulfide)

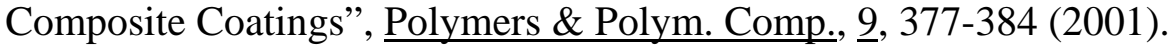

- Sugama, T., Webster, R., Reams, W., and Gawlik, K., "High-performance Polymer Coatings for Carbon Steel Heat Exchanger Tubes in Geothermal Environments”, J. Materials Science, 35, 2145-2154 (2000).

- Sugama, T., "Anti-oxidants for Retarding Hydrothermal Oxidation of Polyphenylenesufide (PPS) Coatings in Geothermal Environments”, Mater. Letters, 43, 185-191 (2000).

- Sugama, T. and Hayenga, P., "Boehmite-reinforced Polyphenylenesulfide as a Wear/Corrosion Resistant Coating”, Polymers \& Polym. Comp. , $\underline{8}$, 307-318 (2000).

- Sugama, T. "Interfaces Between Geothermal Brine-Induced Scales and SiC-filled Polymer linings”, Geothermics, 27, 387-400 (1998).

- Sugama, T., "Polytetrafluoroethylene-blended Polyphenylenesulfide Coatings for Mitigating Corrosion of Steel in Geothermal Environments”, Polymers \& Polym. Comp., $\underline{6}$, 373-385 (1998).

\subsection{Conf. Proceedings}

- Gawlik, K., Sugama, T., and Hirtz, P. “Field Testing and Analysis of Polyphenylenesulfide Composite Coatings Exposed to High Temperature, $\mathrm{pH}-$ modified Brine”, Geothermal Resources Council Transactions, (in press).

- Sugama, T., Gawlik, K., and Hirts, P. "High Temperature Field Testing of Polyphenylenesulfide Composite Coatings”, Geothermal Resources Council Transactions, 28, 450-461 (2004).

- Gawlik, K. and Sugama, T. "Long-term Tests of Polyphenylenesulfide Composite Coatings”, Geothermal Resources Council Transactions, 27, 577-588 (2003).

- Sugama, T. and Gawlik, K. "Filler Materials for Polyphenylenesulfide Composite Coatings”, Geothermal Resources Council Transactions, 25, 41-46 (2001).

- Gawlik, K., Sugama, T., Webster, R. and Reams, W. "Development and Field Testing of Polymer Heat Exchanger Tube Coatings”, Geothermal Resources Council Transactions, 24, 659-664 (2000).

- Gawlik, K., Sugama, T., Webster, R. and Reams, W. "Field Testing of Heat Exchanger Tube Coatings”, Geothermal Resources Council Transactions, 23, 6569 (1999). 
- Gawlik, K., Sugama, T., Webster, R. and Reams, W. "Field Testing of Heat Exchanger Tube Coatings", Geothermal Resources Council Transactions, 22, 385-391 (1998).

\section{Coatings for HX Tube/Sheet and Pipe/Pipe Joint Areas}

Coatings that are curable at low temperature, ranging from $25^{\circ}$ to $80^{\circ} \mathrm{C}$, yet possess high-hydrothermal temperature stability are needed for preventing the corrosion at $\mathrm{HX}$ tube/sheet and pipe/pipes joints after roller expansion or welding joining process. None of the coatings tested can withstand exposure to the high compressive strengths and high temperatures of the joining process. Hence, the joint areas must be recoated with materials possessing the properties described in the Introduction section.

Over the past five years, four candidate coatings, the poly(tetrafluoroethylene)/(hexafluoropropylene) (PTFHFP), NASA-developed polybezimidazole (PBI), polyarylethersulfone (PES), and calcium aluminate cement (CAC)-filled styrene-acrylic latex (SAL), were evaluated to meet the material criteria.

\subsection{PTFHFP Coating}

Room temperature-curable fluorinated ethylene-propylene called polyfluorocarbon is very attractive to use as a tube-end coating because of its excellent thermal stability at temperatures up to $300^{\circ} \mathrm{C}$ [24].

\subsubsection{Experimental}

Organic solvent-based poly-(-tetrafluoroethlene)/(hexaflouoropropylene), the polyfluorocarbon paint, was supplied by the Lauren Manufacturing Company. In an attempt to cure this paint at room temperature, an organic silicon oligomer, obtained from the same company, was used as the catalyst. The catalyst of $7 \%$ by weight of the total mass of the paint was incorporated into the paint, and then blended thoroughly by a stirring rod. The pot life of the catalyzed paint with the viscosity of $\sim 100$ cps was about 2 hours at $25^{\circ} \mathrm{C}$. The 1010 carbon steel panels (size, $6 \mathrm{~cm} \times 6 \mathrm{~cm}$ ) were employed as the substrates. Prior to depositing the paint, the surfaces of the substrates were scoured with an aceton-soaked tissue to remove any contaminants. In preparing the coating film over the cleaned steel surfaces, the steel panels were dipped into the catalyzed paint at room 
temperature, and withdrawn slowly. The paint-wetted panels then were left for 24 hours to allow the paint to form a solid film. This dip-withdraw-curing process was repeated twice more. The average thickness of the film deposited to the panel surface was 0.185 $\mathrm{mm}$. Finally, the coated panels were exposed for up to 20 days in a $200^{\circ} \mathrm{C}$ autoclave containing a $13 \% \mathrm{NaCl}$ solution and 20,000 $\mathrm{ppm} \mathrm{CO}_{2}$.

\subsubsection{Results}

The room-temperature curable PTFHFP coatings adequately protected carbon steel against corrosion in a short-term autoclave exposure test in which they were exposed to 20,000 ppm $\mathrm{CO}_{2}$-laden $13 \% \mathrm{NaCl}$ solution at $200^{\circ} \mathrm{C}$. However, their surfaces underwent some degree of hydrothermal oxidation during exposures for up to 20 days. The incorporation of oxygen into the coating surfaces during exposure led to the replacement of $\mathrm{F}$ atoms in the polyfluorocarbon structure for oxygen atoms, thereby causing the scission and breakage of the backbone C-C linkages in the following two-step hydrothermal oxidation schemes;
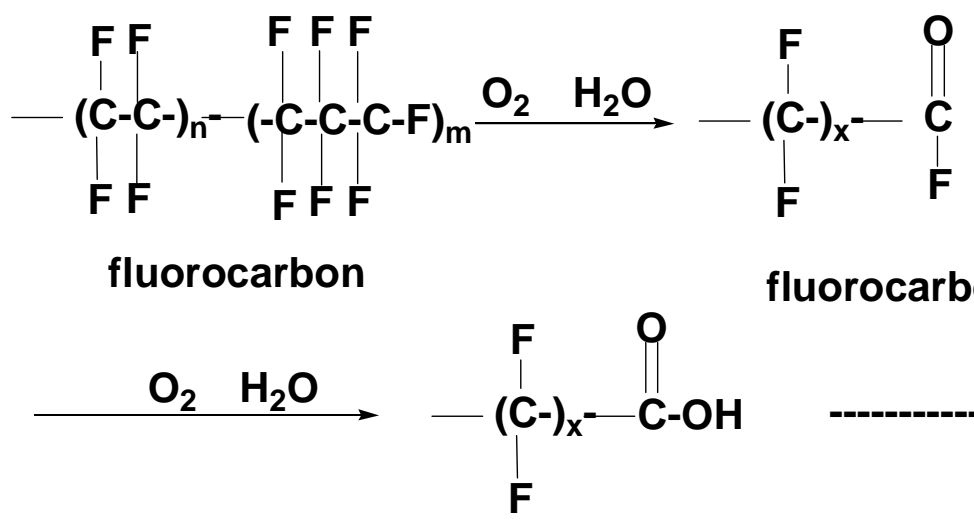

fluorocarbonyl

\section{fluorocarboxylic acid}

Such oxidation damage to the molecular structure generated undesirable hydrophilic fluorocarboxylic acid derivatives, which enhances the wetting of coating surfaces by water, thereby lowering their water-repellent properties. The derivatives also promoted the uptake of moisture by the coatings. Substituting fluorocarbons by fragmental fluorocarboxylic acid not only reduced the extent of the coating's crystallinity, but also was detrimental to the thermal stability of the coatings. In fact, the thermal 
decomposition temperature of fluorocarboxylic acid began at $\sim 170^{\circ} \mathrm{C}$, which was $140^{\circ} \mathrm{C}$ lower than that for fluorocarbon.

Correspondingly, the ionic conductivity of coating layer increased with an extending exposure time; namely, the pore resistance of the unexposed coatings fell by a magnitude of four orders to $2.0 \times 10^{6} \mathrm{ohm}-\mathrm{cm}^{2}$ after a 20-day exposure.

\subsection{PBI Coating}

Polybenzimidazole (PBI) is a hard glassy polymer possessing outstanding thermal stability, including nonflammability, a high heat-deflection temperature of $435^{\circ} \mathrm{C}$, and an upper working temperature of $260-400^{\circ} \mathrm{C}$. Currently, fibrous materials made from PBI are widely applied to fire-resistant protective gears, firemen's turnout coats, astronaut's space suits and industrial workers' suits [25]. Also, it is becoming increasingly attractive as high temperature membrane for solid polymer fuel cells that reliably operate at temperatures up to $200^{\circ} \mathrm{C}$ [26-30]. However, there has been virtually no report on its applicability as an anti-corrosion coating under hydrothermal conditions.

\subsubsection{Experimental}

A 26wt\% polybenzimidazole (PBI) dissolved in a 68wt\% dimethyl acetamide (DMAC) solvent containing 6wt\% $\mathrm{LiCl}$ stabilizer was supplied by Celanese Acetate A.G. Corporation. The metallic substrate used was commercial AISI 1008 carbon steel. The surfaces of cleaned carbon steel were directly coated with a PBI-dissolved DMAC solution using a paint blush, and then left for 24 hours at ambient temperature to allow most of the DMAC to evaporate. After 24 hours, the PBI solution covering the surfaces of the metal had become converted into a solid film. To ensure that the coatings to be tested did not include any DMAC solvent, the PBI-coated steel panels were heated for 15 hours in an air oven at $80^{\circ} \mathrm{C}$. This coating process was repeated three times to make a film of $~ 6$ mil thickness. The PBI-coated steel panels (62.5 $\mathrm{mm}$ x $62.5 \mathrm{~mm})$ then were exposed for up to 14 days in an autoclave containing a $\mathrm{CO}_{2}$-laden brine $(0.5 \mathrm{wt} \%$ sodium hydrogen carbonate, $13 \mathrm{wt} \%$ sodium chloride, and $86.5 \mathrm{wt} \%$ water) at $300^{\circ} \mathrm{C}$ under a hydrothermal pressure of $8.27 \mathrm{MPa}$. 


\subsubsection{Results}

The PBI film derived from the solvent-based precursor displayed a great thermal stability at heat temperatures up to $600^{\circ} \mathrm{C}$. However, the imidazole rings within the PBI's molecular structure were vulnerable to hydrolysis in a brine at $300^{\circ} \mathrm{C}$, thereby promoting the rupture of their $=\mathrm{N}=\mathrm{C}$ and $/ \mathrm{C}-\mathrm{N}=$ bonds. As seen in the hot brine-induced hydrolysis mechanism below, the hydrolysis led to the formation of two hydrolysate derivatives, biphenyl tetra-amine and benzodicarboxylic acid, causing a decline in its thermal stability and an increase in the susceptibility of coating's surface to moisture. Such shortcoming of PBI coating in the hydrothermal environments diminished its maximum effectiveness in protecting the metal against corrosion. Thus, when the PBIcoated carbon steel panels were exposed to $300^{\circ} \mathrm{C}$ brine, the uptake of corrosive electrolyte by PBI coating progressively increased as its exposure time was extended. After 14 days, the coating film was delaminated from the underlying steel surfaces. Therefore, PBI is an inadequate material for use as a corrosion-preventing coating in geothermal environments at a temperature of $300^{\circ} \mathrm{C}$.
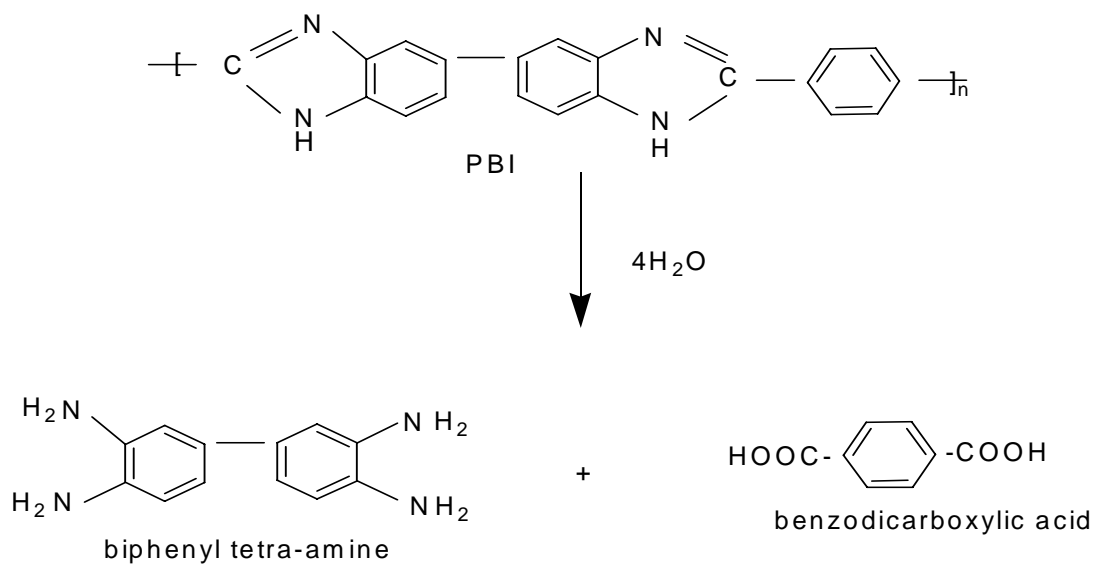

\subsection{PES Coating}

Among the high-performance polyaryl-based engineering polymers, polyethersuflone (PES) is known to possess excellent thermal properties including the deflection temperature of $200^{\circ} \mathrm{C}$ under a pressure of $1.8 \mathrm{MPa}$, a high glass transition 
temperature of $\sim 230^{\circ} \mathrm{C}$, and processing temperature of $\sim 320^{\circ} \mathrm{C}$ [31]. Although it is well documented that exposure to UV radiation causes photo-degradation of the PES film [32,33], two critical factors, its stability at high temperature and its solubility in an organic solvent at relatively low temperature of $90^{\circ} \mathrm{C}$, urged us to evaluate its potential as a low-temperature curable, corrosion-preventing coating for the joint areas in a hot brine environment.

\subsubsection{Experimental}

Solvay Advanced Polymers, LLC., supplied the polyethersulfone (PES) amorphous polymer as a powder. The N-methyl pyrrolidone (NMP) solvent used to dissolve the PES was obtained from Lyondell Chemical Corp. Assuming that the surface of joint areas is prepared by sand blasting, the sand blasted 1010 carbon steel coupons, $6.5 \mathrm{~cm} \times 6.5 \mathrm{~cm}$, were employed as the substrates. The PES powder was dissolved in the NMP solvent at $100^{\circ} \mathrm{C}$, and the precursor solution was left for 24 hours in an ambient temperature before using it. Four formulations were prepared as the solvent-based coatings with PES/NMP weight ratios of 5/95, 10/90, 15/85, and 20/80. The surfaces of the coupons were coated with a PES-dissolved NMP solution using a paintbrush, and then left for 24 hours at ambient temperature to allow most of the NMP to evaporate; after this time, the PES solution covering the surfaces of the metal had become converted into a solid film. To ensure that these coatings did not retain any NMP solvent, the PEScoated steel panels were heated for 10 hours in an air oven at $80^{\circ} \mathrm{C}$. This coating process was repeated four times to make films $\sim 25 \mu \mathrm{m}$ thick for the 5/95 ratio, $\sim 48 \mu \mathrm{m}$ for the $10 / 90$ ratio, $\sim 74 \mu \mathrm{m}$ for the 15/85 ratio, and $\sim 100 \mu \mathrm{m}$ for the $20 / 80$ ratio. The coated coupons were exposed for up to 15 days in an autoclave containing a $\mathrm{CO}_{2}$-laden brine (0.5 wt.\% sodium hydrogen carbonate, $13 \mathrm{wt} \%$ sodium chloride, and $86.5 \mathrm{wt} \%$ water) at $200^{\circ}, 250^{\circ}$, and $300^{\circ} \mathrm{C}$.

\subsubsection{Results}

Among a series of amorphous polyethersulfone (PES) polymer-dissolved N-methyl pyrrolidone (NMP) solvent precursor systems, one consisting of 15wt\% PES and 85wt \% NMP proved to be the most effective in fabricating a coating film for protecting carbons 
steel against corrosion. When the PES films were exposed in brine at $200^{\circ}, 250^{\circ}$, and $300^{\circ} \mathrm{C}$, their surfaces underwent hydrothermal oxidation. At $200^{\circ} \mathrm{C}$, the incorporation of some oxygen into the PES structure led to the rupture of sulfone linkages within PES, causing the formation of intermediate benzosulfone hydroperoxide, which was converted eventually into benzosulfonic acid as the oxidation derivative. Increasing the brine temperature to $250^{\circ} \mathrm{C}$ that incorporated more oxygen, not only markedly promoted the sulfone $\rightarrow$ benzosulfonic acid conformational transformation, but also brought about the scissure of $\mathrm{C}-\mathrm{C}, \mathrm{C}=\mathrm{C}$, and $\mathrm{C}-\mathrm{H}$ bonds in the phenyl rings, thereby resulting in their opening. In addition, a substantial number of the ether linkages within PES were broken as the temperature was further increased to $300^{\circ} \mathrm{C}$;

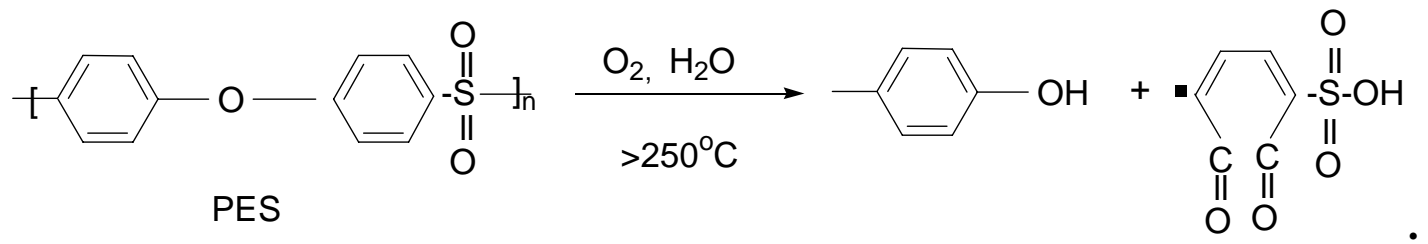

This breakage promoted the ether $\rightarrow$ benzophenol-type oxidation derivative transformation, while the two other oxidation-associated conformational changes, sulfone $\rightarrow$ benzosulfonic acid and ring opening, still continued. In considering the corrosionpreventing performance of coatings, the considerable attention must be paid to these oxidation derivatives and to ring opening that generates the functional hydroxyl and carbonyl groups because they both enhance the magnitude of susceptibility of the coatings' surfaces to moisture. Thus, in a $200^{\circ} \mathrm{C}$ brine environment, PES coating of $\sim 74$ $\mu \mathrm{m}$ thick adequately protected the carbon steel against corrosion because of minimum hydrothermal oxidation. In contrast, at $\geq 250^{\circ} \mathrm{C}$, the maximum efficacy of the coatings in preventing the corrosion was significantly diminished by their severe oxidation.

\subsection{CAC-filled SAL Coating}

In our two previous studies on the effectiveness of hydraulic cement-type fillers in improving the hydrothermal stability of styrene/acrylonitril/trimethylopropane trimethacrylate and polyester/styrene copolymers [34-36], we incorporated an appropriate 
amount of calcium silicate cement (CSC) into the copolymer. The hydrothermal reaction between the Ca ions liberated from CSC filler and the ester groups in copolymer at $240^{\circ} \mathrm{C}$ led to the formation of Ca-complexed carboxylate compounds that were responsible for improving the hydrothermal stability of this copolymer.

On the other hand, a mixture of water-borne latex materials, such as the poly(vinyl acetate), polychloroprene, polymethacrylates, poly(vinyl choloride) and the copolymers of polystyrene, polybutadiene, and polyacrylonitirile, made by emulsion polymerization, is widely used in hydraulic cements to improve their mechanical properties, chemical resistance, and water proofing.

Based upon information described above, our particular interest is to assess the potential of mixed slurries of styrene acrylic latex and calcium aluminate cement (CAC) filler instead of using CSC as room temperature-curable high temperature performance coatings for joint areas.

\subsubsection{Experimental}

Noveon, Inc supplied the water-borne styrene acrylic latex (SAL, Trade Name, HYCA®26-1265). Calcium aluminate cement (CAC, Secar 60) used as the hydraulic filler was obtained from the Lafarge Aluminate Corp. The CAC contained two major oxides, 69.8-72.2 \% aluminum oxide and 26.8-29.2 \% calcium oxide. The filler had a surface area of 3800-4400 cm2/g, and a particle size of $<90 \mu \mathrm{m}$. An x-ray diffraction (XRD) analysis revealed that mineralogically it consisted of crystalline calcium monoaluminate ( $\left.\mathrm{CaO} . \mathrm{Al}_{2} \mathrm{O}_{3}, \mathrm{CA}\right)$ as the principal component, calcium dialuminate $\left(\mathrm{CaO} .2 \mathrm{Al}_{2} \mathrm{O}_{3}, \mathrm{CA}_{2}\right)$ as the secondary component, and pervoskite $\left(\mathrm{CaTiO}_{3}\right)$ as the minor one. The metallic substrate used was commercial AISI 1008 carbon steel. Five slurries made with SAL/CAC ratios of 100/0, 80/20, 60/40, 40/60, and 30/70, by weight, were evaluated. The slurries were prepared in a shear-blender to uniformly distribute the CAC filler in the latex medium. In preparing the test samples, the slurries were directly painted on to the alkali-cleaned carbon steel coupons ( $65 \mathrm{~mm}$ x $65 \mathrm{~mm}$ ), and then left for 24 hours at room temperature to solidify the slurry. This coating process was repeated several times to obtain coating thicknesses ranging from 0.9 to $1.2 \mathrm{~mm}$. For comparison, CAC slurry made with a water/CAC ratio of 0.5 also was deposited on the steel's surface. 
The coated coupons then were exposed for up to 21 days in an autoclave containing 0.05 $\mathrm{M} \mathrm{Na}_{2} \mathrm{CO}_{3}$-laden water at $250^{\circ} \mathrm{C}$.

\subsubsection{Results}

When water-borne styrene acrylic latex (SAL) was mixed with the calcium aluminate cement (CAC) as hydraulic filler at room temperature, the setting time of SAL depended on the content of CAC; namely, an increase in its content shortened the pot-life of SAL. For instance, the setting time of the slurry made with the SAL/CAC ratio of 30/70 by weight, which was the highest content of CAC in this test series, was only a 100 min after mixing these two components. The decreasing content of CAC, as in the 40/60 ratio slurry, extended setting time to $\sim 420 \mathrm{~min}$, corresponding to $\sim 320$ min delay compared with that of the 30/70 ratio. Among the 100/0, 80/20, 60/40, 40/60, and 30/70 ratios, the surface of the slurry made with the 30/70 ratio was converted into the solid state within 240 min at room temperature. This mixture exhibited the lowest magnitude of susceptibility to moisture, suggesting that the surface of the SAL coating became more hydrophobic as the content of CAC was increased.

In the $\mathrm{CO}_{2}$-laden hydrothermal environment at $250^{\circ} \mathrm{C}$, the carboxylic acid, $\mathrm{COOH}$, groups within SAL hydrothermally reacted with the $\mathrm{Ca}^{2+}$ and $\mathrm{OH}^{-}$ions dissociated from the hydrolysis of CAC to form the Ca-complexed carboxylate groupcontaining SAL (Ca-CCG-SAL). Concurrently, the reaction between $\mathrm{Ca}^{2+}$ and $\mathrm{CO}_{3}{ }^{2-}$ led to the formation of crystalline calcite, $\mathrm{CaCO}_{3}$. These two reactions caused the depletion of $\mathrm{Ca}$ in the two calcium aluminate reactants in the CAC, calcium monoaluminate $\left(\mathrm{CaO} . \mathrm{Al}_{2} \mathrm{O}_{3}, \mathrm{CA}\right)$ and calcium dialuminate $\left(\mathrm{CaO} .2 \mathrm{Al}_{2} \mathrm{O}_{3}, \mathrm{CA}_{2}\right)$. The Ca-destitute $\mathrm{CA}$ and $\mathrm{CA}_{2}$ then were transformed into crystalline boehmite, $\gamma$-AlOOH. The carboxylic acid $\rightarrow$ Ca-complexed carboxylate group transition was completed in the first two hours of autoclave exposure. In contrast, the $\mathrm{CaCO}_{3}$ and beohmite crystals grew continuously throughout a 21-day autoclaving period.

The slurry made with 30/70 ratio displayed a great potential as a room temperature-curable anti-corrosion coating for heat exchanger/sheets and pipe/pipe joints. The following three major factors of this coating contributed significantly to mitigating the corrosion of carbon steel in $\mathrm{CO}_{2}$-laden geothermal environment at temperatures up to 
$250^{\circ} \mathrm{C}$ : First was the enhancement of the thermal stability of bulk SAL from $320^{\circ} \mathrm{C}$ to $415^{\circ} \mathrm{C}$ by its transformation into the Ca-CCG-SAL polymer; second was the conversion of the porous structure in the non-autoclaved coating into the densified one due to the growth of crystalline calcite and boemite with increasing autoclave time; and, third was its good adherence to the steel's surface. The integrity of these three factors provided a minimum uptake of corrosive electrolytes by the coating and the inhibition of the cathodic oxygen reduction reaction at the corrosion site of steel. In the former, the extent of corrosive ionic conductivity through the coating layer was considerably reduced as the autoclaving time was extended (Figure 37). This fact strongly demonstrated that this SAL/CAC hybrid coating system has a self-advancing characteristic that enhances its ability to protect the steel against corrosion during autoclaving.

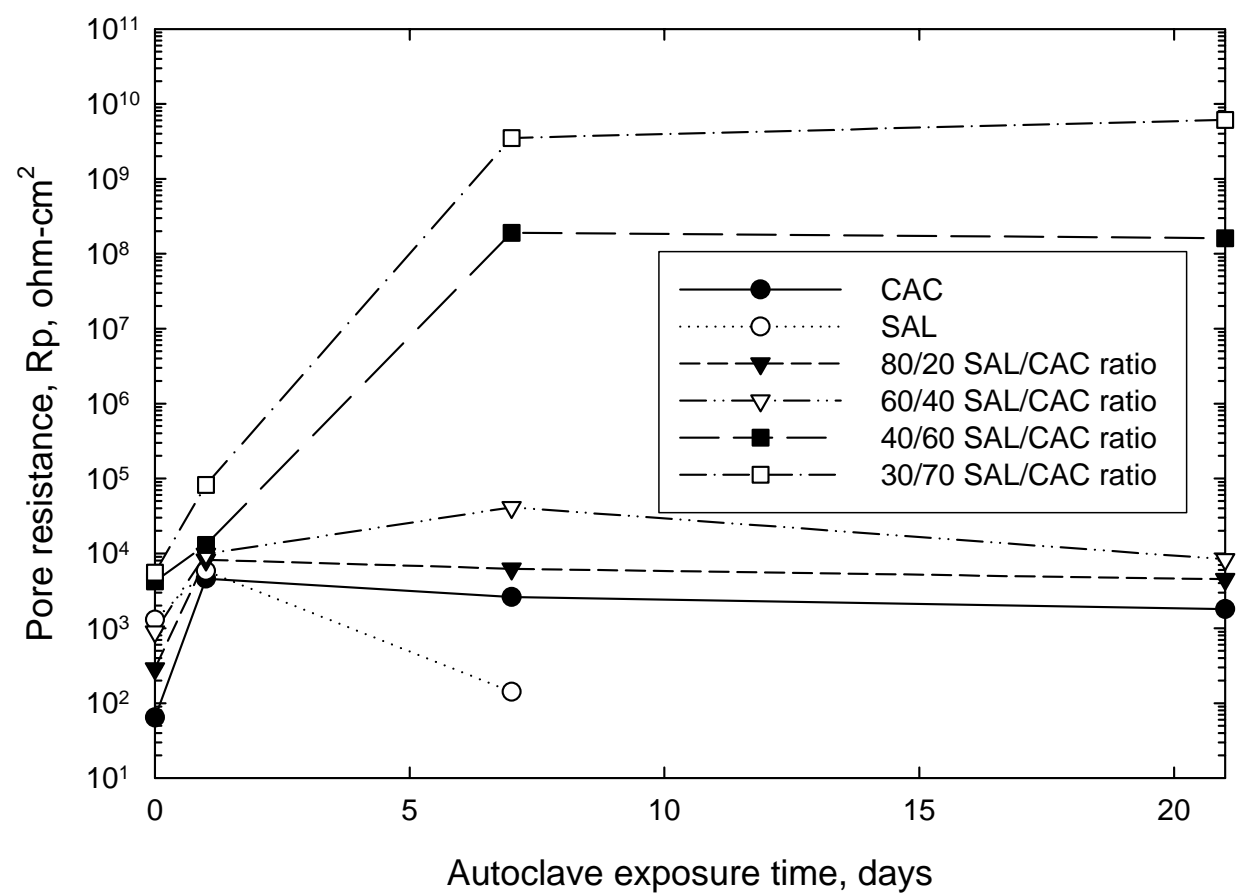

Figure 37. Pore resistance, $\mathrm{Rp}$, of various coatings plotted as a function of autoclaving time.

\subsection{Three-mo.-Long Field Exposure Tests}

Based upon the information described above, two potential coating systems, PES and CAC-filled SAL, were selected to conduct a short-term field validation test at Mammoth Power Plant operating at brine temperature of $\sim 160^{\circ} \mathrm{C}$. To conduct this field test, the 
carbon steel coupons (1-in. wide x 3-in. long) were coated with these materials. The thickness of coatings ranged from 0.3 to $0.8 \mathrm{~mm}$. The results revealed that the PES coating failed after three months exposure; in contrast, the CAC-filled SAL coating withstood this geothermal environment.

\subsection{Conclusions}

1. The appearance of room-temperature-curable poly(tetrafluoroethylene) / (hexafluoropropylene) (PTFHFP) coating after 20 days exposure to $200^{\circ} \mathrm{C}$ brine revealed that they adequately protected the underlying steel substrate against corrosion in this short-term exposure. However, the superficial layer of coating suffered hydrothermal oxidation, leading to conformational transformation of the fluorocarbon structure into a fragmental fluorocarboxylic acid derivative as the oxidation reaction product. This transformation generated three undesirable features; 1) an increase in the susceptibility of the coating surfaces to moisture, 2) a decline in the thermal stability of the coatings, and 3) a reduction of the crystallinity of the polymer.

2. A room-temperature film-forming polybenzimidazole (PBI) coating displayed thermal stability at temperatures up to $600^{\circ} \mathrm{C}$. However, when its film was exposed to $300^{\circ} \mathrm{C}$ brine, it underwent hydrolysis. This shortcoming lowered the film's maximum effectiveness in minimizing the rate of transportation through it of corrosive electrolytes in hydrothermal environments. As a result, the PBI coating film was hydrothermally degraded, and it delaminated from the underlying steel substrates after a 14-day exposure.

3. $\mathrm{A} \sim 75 \mu \mathrm{m}$ thick polyethersulfone (PES) coating performed well in protecting the steel against corrosion in brine at $200^{\circ} \mathrm{C}$. However, at $\geq 250^{\circ} \mathrm{C}$, the PES underwent severe hydrothermal oxidation that caused the cleavage of sulfone- and ether-linkages, and the opening of phenyl rings. These, in turn, led to sulfone $\rightarrow$ benzosulfonic acid and ether $\rightarrow$ benzophenol-type oxidation derivative transformations, and the formation of carbonyl-attached open rings, thereby resulting in the incorporation of the functional groups, hydroxyl and carbonyl, into the coating. The presence of these functional groups raised concerns about 
the diminutions in water-shedding and -repellent properties that are important properties of the anti-corrosion coatings; such changes were reflected in an enhancement of the magnitude of susceptibility of the coatings' surfaces to moisture. Consequently, the disintegration of the PES structure by hydrothermal oxidation was detrimental to the maximum efficacy of the coating in protecting the steel against corrosion, allowing the corrosive electrolytes to infiltrate easily through it.

4. Hydrothermally self-advancing hybrid material, which was prepared by blending two starting materials, water-borne styrene acrylic latex (SAL) as the matrix and calcium aluminate cement (CAC) as the hydraulic filler, was identified as the most promising room-temperature curable anti-corrosion coating for $\mathrm{HX} /$ sheet and pipe/pipe joint areas in $\mathrm{CO}_{2}$-laden geothermal environments at $250^{\circ} \mathrm{C}$. The following two major factors supported the self-improving mechanisms of the coating during its exposure in an autoclave: First was the formation of a high temperature stable polymer structure of Ca-complexed carboxylate groups containing SAL (Ca-CCG-SAL) due to hydrothermal reactions between SAL and CAC; second was the growth with continuing exposure time of crystalline calcite and boehmite phases coexisting with Ca-CCG-SAL. These two factors promoted the conversion of the porous microstructure in the non-autoclaved coating into a densified one after 7 days exposure. The densified microstructure not only considerably reduced the conductivity of corrosive ionic electrolytes through the coatings' layers, but also contributed to the excellent adherence of the coating to underlying steel's surface that, in turn, retarded the cathodic oxygen reduction reaction at the corrosion site of steel. Such characteristics including the minimum uptake of corrosive electrolytes by the coating and the retardation of the cathodic corrosion reaction played an important role in inhibiting the corrosion of carbon steel in geothermal environments.

\subsection{Publications}

- Sugama, T. "Hydrothermally self-advancing Hybrid Coatings for Mitigating Corrosion of Carbon Steel”, BNL publication (in press). 
- Sugama, T. "Polyethersulfone Coating for Mitigating Corrosion of Steel in Geothermal Environemt”, BNL publication (in press).

- Sugama, T., "Hydrothermal Degradation of Polybenzimidazole Coating”, Matter Letters, 58, 1307-1312 (2004).

- Sugama, T. and Gawlik, K., "Poly(tetrafluoroethlyene)/(hexafluoropropylene) Coatings for Mitigating the Corrosion of Steel in a Simulated Geothermal Environment”, Int. J. Prog. In Organic Coatings, 42, 202-208 (2001).

\section{Coatings for Wellhead Components}

As described in the Introduction, the wellhead components encountered very harsh environment with a flow velocity of $\sim 3 \mathrm{~m} / \mathrm{sec}$ of brine at $250^{\circ} \mathrm{C}$. If their components were made of inexpensive carbon steel instead of titanium alloy-based metals, $250^{\circ} \mathrm{C}$ hydrothermal stable coatings would be needed to protect the component's surface against corrosion. Thus far, three different coatings were evaluated: One was the titanium carbonitride [(Ti(CN)] coatings by chemical vapor deposition (CVD) technology; the second was the nickel-aluminum ( $\mathrm{Ni}-\mathrm{Al}$ ) coatings by flame-spray technology; and the third was the PPS/Clay coatings by nanocomposite technology.

\subsection{CVD-Titanium Carbonitride}

Non-oxide titanium nitride (TiN) and titanium carbide (TiC) coatings deposited by chemical vapor deposition (CVD) are currently used as a protective barrier in machine components because of their great wear and chemical resistances as well as their excellent thermal stability [37]. Considering the coating technologies, one of the major advantages of CVD is the fact that there is no requirement for sintering in fabricating non-oxide ceramic films. The films are directly deposited onto the metal surfaces by phase transformation from the raw vapors or gases to the solid in a hot-wall reactor. The thickness of the films is one important key to improving their ability to protect the metal substrates against wear, oxidation, and corrosion; increasing the coating's thickness extends their useful life as a protective barrier.

$\mathrm{CVD}$ at a moderate temperature between $700 \mathrm{E}$ and $900^{\circ} \mathrm{C}$ can fabricate thicker coating layers compared with those produced by conventional CVD-processes at a higher 
deposition temperature of $>$ 900EC. This technology called moderate temperature-CVD (MT-CVD) significantly extended their potential application in protecting machine components, such as carbide milling inserts, carbide cutting tools, saws and blades; a lower temperature mitigates thermal stress on metal substrates, leading to the possibility of depositing thicker coating layers [38].

Thus, the objective of the present study was to assess the usefulness of the MTCVD-derived titanium carbonitride, $\mathrm{Ti}(\mathrm{CN})$, coatings consisting of the combined phases of $\mathrm{TiN}$ and $\mathrm{TiC}$, as corrosion/abrasive wear-resistant barriers for mild carbon steels in a low $\mathrm{pH}$, hypersaline brine geothermal environment at a hydrothermal temperature of 250EC.

\subsubsection{Experimental}

The MT-CVD Ti(CN) coated specimens were prepared by Sylvester Bernex Company, under the following deposition conditions: The CRS panels, $6.25 \times 6.25 \mathrm{~cm}$ in size and $1 \mathrm{~mm}$ in thickness, to be coated were held in an externally heated retort called a hot-wall reactor. The acetonitrile $\left(\mathrm{CH}_{3} \mathrm{CN}\right)$-titanium tetrachloride $\left(\mathrm{TiCl}_{4}\right)$ system was used as a precursor for depositing $\operatorname{Ti}(\mathrm{CN})$ onto CRS surfaces. The vaporized mix precursor was introduced through a gas distribution system into the reactor. The flow velocity of reactive chemical vapor was adjusted by controlling the flow rate of the $\mathrm{H}_{2}$ carrier gas. All the depositions were made in the range of $700 \mathrm{E}$ to $900 \mathrm{EC}$ under a pressure of $\sim 0.03 \mathrm{MPa}$. The chemical reaction occurring between these gaseous phases to deposit $\mathrm{Ti}(\mathrm{CN})$ onto the CRS surfaces may be hypothetically expressed as $2 \mathrm{TiCl}_{4}+$ $\mathrm{CH}_{3} \mathrm{CN}+9 / 2 \mathrm{H}_{2} 6 \mathrm{TiN}+\mathrm{TiC}+\mathrm{CH}_{4}+8 \mathrm{HCl}$ [37]. Two different thicknesses, 4 and 12 $\mu \mathrm{m}$, of the coating films were deposited. The coated and uncoated CRS panels were exposed in an autoclave containing a $\mathrm{pH} 1.68$, brine $\left(1 \mathrm{wt} \% \mathrm{H}_{2} \mathrm{SO}_{4}-13 \mathrm{wt} \% \mathrm{NaCl}-86\right.$ wt\% water) at temperature of 250EC under a pressure of $1.45 \mathrm{MPa}$.

\subsubsection{Results}

Although the effectiveness of $\mathrm{Ti}(\mathrm{CN})$ in mitigating the rate of corrosion of steel depended on its thickness , there were two other critical factors: First, Ti(CN) was susceptible to oxidation reactions with aqueous steam. This reaction led to the 
transformation of $\mathrm{Ti}(\mathrm{CN})$ into $\mathrm{TiO}_{2}$ scales. Furthermore, the $\mathrm{TiO}_{2}$ scales had chemical affinity for $\mathrm{HCl}$ yielded by a reaction between $\mathrm{H}_{2} \mathrm{SO}_{4}$ and $\mathrm{NaCl}$ in acid brine, forming a water-soluble $\mathrm{TiCl}_{4}$ salt, which dissolves in the aqueous media. Thus, the hot acid brine corroded the $\mathrm{Ti}(\mathrm{CN})$ coatings through the processes of oxidation $\left(\mathrm{TiO}_{2}\right) 6$ chlorination $\left(\mathrm{TiCl}_{4}\right) 6$ dissolution. Second, there existed inherent defects and pores in the CVDderived coating layers, allowing the corrosive species to infiltrate easily. In fact, the growth of steel's corrosion products, such as $\mathrm{Fe}_{2} \mathrm{O}_{3}, \mathrm{FeCl}_{3}$, and $\mathrm{Fe}_{3} \mathrm{O}_{4}$, beneath the coating layers caused delamination and buckling of the coatings.

\subsection{Flame-sprayed Nickel-Aluminum (Ni-Al)}

Nickel-aluminum (Ni-Al) coatings applied by flame-spray coating technology are very attractive because they not only bond well to the metal surfaces, but also exhibit good plastic deformation, great wear resistance, a coefficient of thermal expansion similar to that of steels, and excellent thermal conductivity [39,40].

The emphasis in this present work was directed toward assessing the potential application of flame-sprayed Ni-Al coating to the wellhead components. The research therefore focused on investigating the changes in chemistry and microstructure of the Ni$\mathrm{Al}$ coating after exposure in the simulated geothermal environment. The coated carbon steel panels were exposed to a corrosive solution consisting of $1.0 \mathrm{wt} \% \mathrm{H}_{2} \mathrm{SO}_{4}, 13.0 \mathrm{wt} \%$ $\mathrm{NaCl}$, and $86.0 \mathrm{wt} \%$ water at 250EC.

\subsubsection{Experimental}

The metallic substrate used was a mild carbon steel (MCS). SW 3670 wires (ASB Industries, Inc. Orberton, $\mathrm{OH}$, with a nickel/aluminum ratio of $80 / 20$ by weight were used as the starting material for the $\mathrm{Ni}-\mathrm{Al}$ coatings. The coatings were applied using a handheld wire flame gun (Model 12E Perkin-Elmer’s Metco Westbury, NY) under an air

pressure of 0.45 MPa onto MCS specimens of 6.25 x $6.25 \mathrm{~cm}$. Before spraying, the surfaces of MCS were cleaned and decontaminated by blasting them with silica grits with a particle-size range 0.177 to $0.297 \mathrm{~mm}$. The average depth of surface roughness of gritblasted MCS, determined by a dektak surface profile measuring system (Sloan Technology Co., Santsa Barbara, CA), ranged from 0.02 to $0.045 \mathrm{~mm}$. Once the wire 
reached its molten temperature in the propane flame, the molten $\mathrm{Ni}-\mathrm{Al}$ particles were projected from a target distance of $10 \mathrm{~cm}$ onto the MCS surfaces. At room temperature, the molten Ni-Al into was completely converted into a solid coating within 1 min after spraying. The average thickness of this coating was $0.09 \mathrm{~mm}$. The x-ray diffraction analysis of this coating layer revealed that the major phase composition in the layers consisted of the two metal components, $\mathrm{Ni}$ and $\mathrm{Al}$. The coated test panels were exposed for up to 14 days in autoclave containing a low $\mathrm{pH}$, hypersaline brine solution (1 wt\% $\mathrm{H}_{2} \mathrm{SO}_{4}, 13 \mathrm{wt} \% \mathrm{NaCl}$, and $86 \mathrm{wt} \%$ water) at $250^{\circ} \mathrm{C}$.

\subsubsection{Results}

The inherent open pores and void spaces present in the Ni-Al coating layers (Figure 38) was a critical issue in protecting the underlying steel substrates against corrosion, allowing the corrosive ionic species to permeate the coating layer. In fact, the coating panels after exposure for 14 days to a hot brine solution at 250EC developed corrosion-generated stress cracks at the interfacial contact zone between the $\mathrm{Ni}-\mathrm{Al}$ and steel (Figure 39). In addition, the coating suffered oxidation during exposure, leading to the growth of $\mathrm{Al}_{2} \mathrm{O}_{3}$ as the major scale compound, and $\mathrm{NiO}$ as the minor one (Figure 40). 


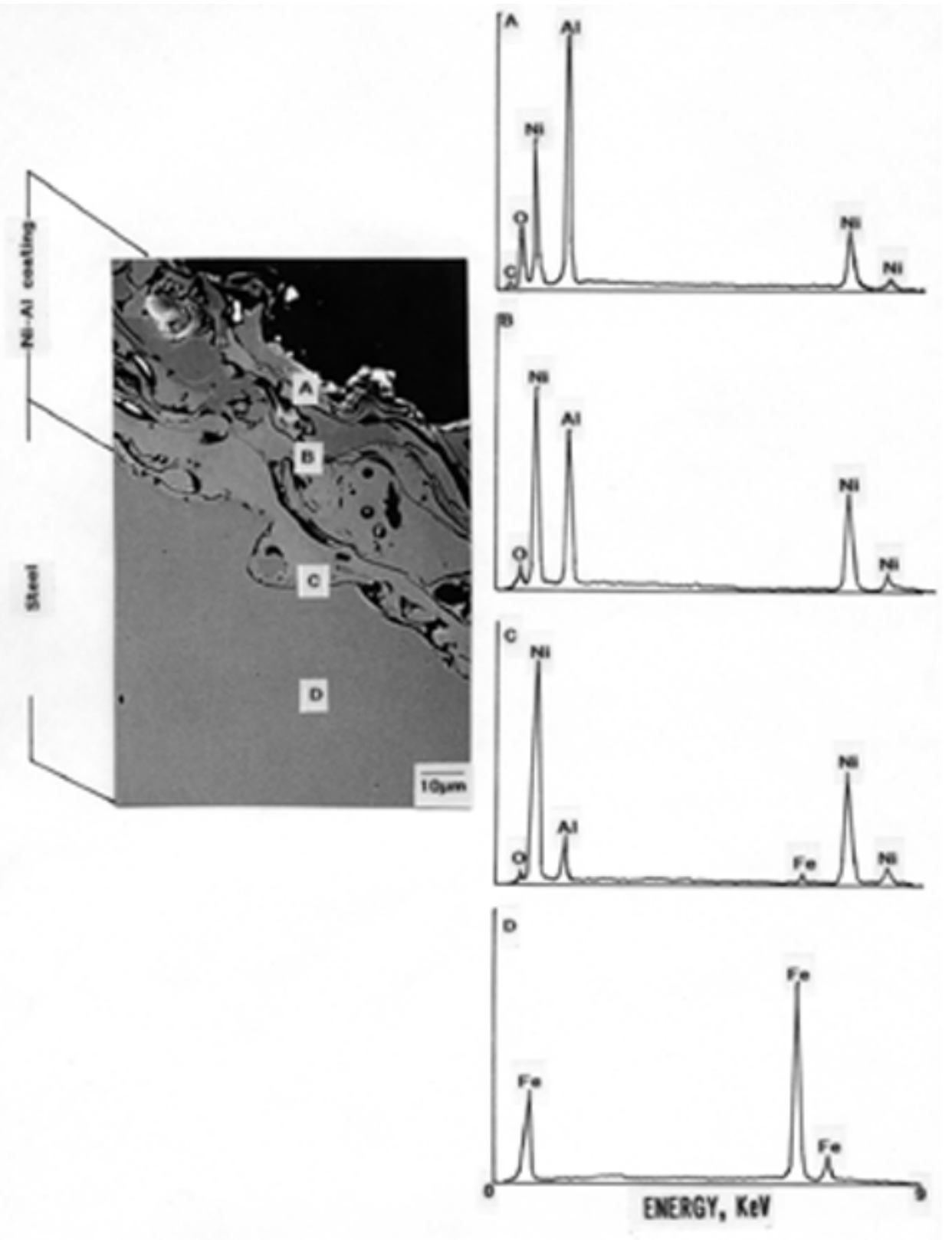

Figure 38. SEM image coupled with (A-D) EDX spectra for a cross-sectional area of the Ni-Al-coated steel before exposure. 


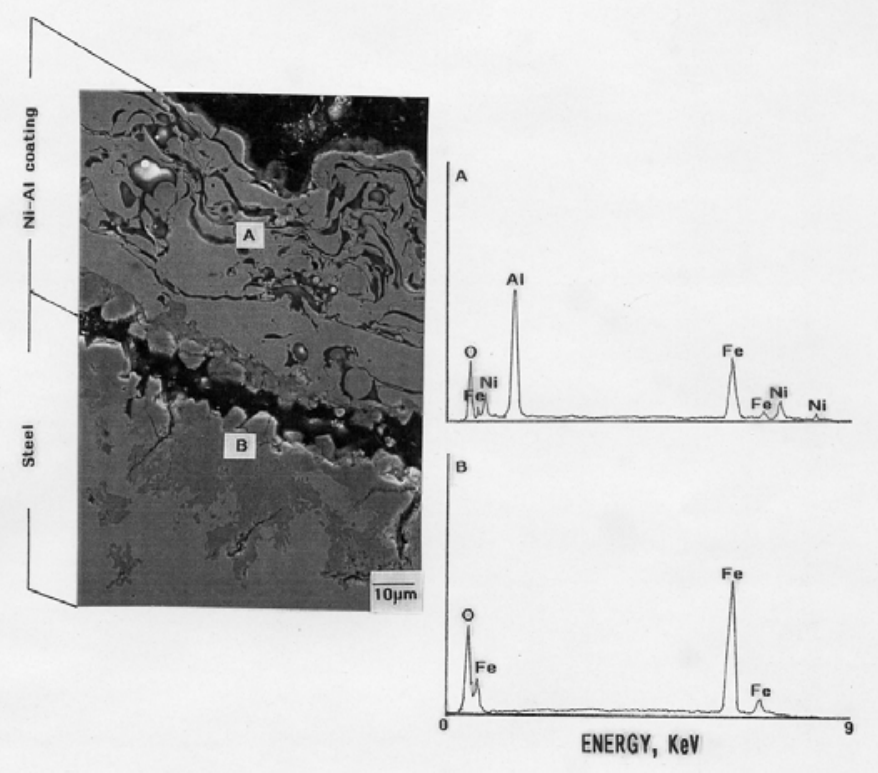

Figure 39. SEM-EDX analyses of cross-sectional area in the Ni-Al/steel joint after exposure for 14 days; EDX for areas (A) and (B).

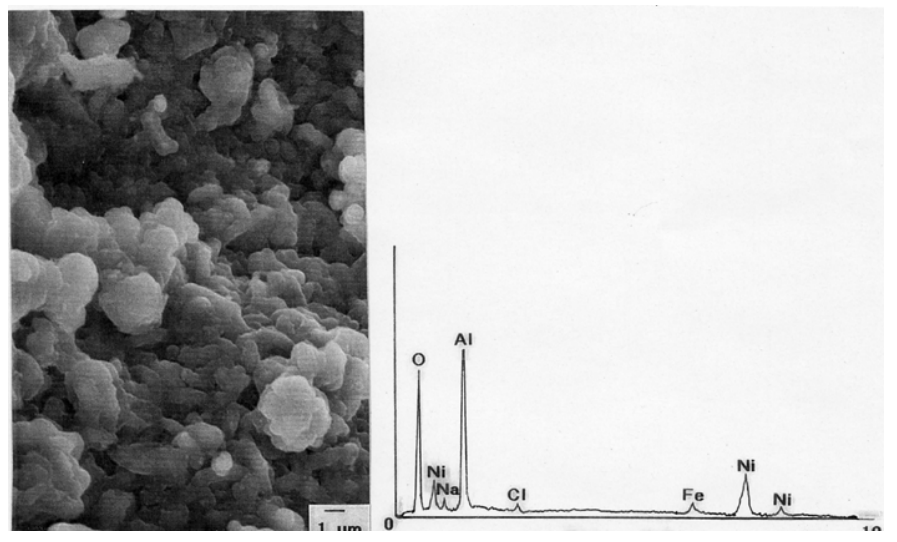

Figure 40. SEM-EDX for the surface of 14 days exposed Ni-Al coating.

\subsection{PPS/Clay Nanocomposite}

Since the melting temperature of PPS is around $250^{\circ} \mathrm{C}$, it is reasonable to assume that this material is able to withstand the brine temperatures up to $200^{\circ} \mathrm{C}$. Thus, our next 
biggest challenge was to apply this composite to the carbon steel plant components intended to be used at higher brine temperatures above $250^{\circ} \mathrm{C}$. We previously studied the hydrothermal stability of this composite coating autoclaved at $250^{\circ} \mathrm{C}$ [41]. The results revealed that the PPS underwent a hot brine-induced oxidation, thereby changing its molecular sulfide bridges to sulfone ones (Figure 41). This alteration allowed corrosive ionic species to permeate gradually through the oxidized PPS with increasing exposure time. Undoubtedly, increasing the melting temperature of PPS is an inevitable next step, if its potential application is targeted towards protecting carbon steel components at $\geq$ $250^{\circ} \mathrm{C}$ against corrosion and scale deposition.

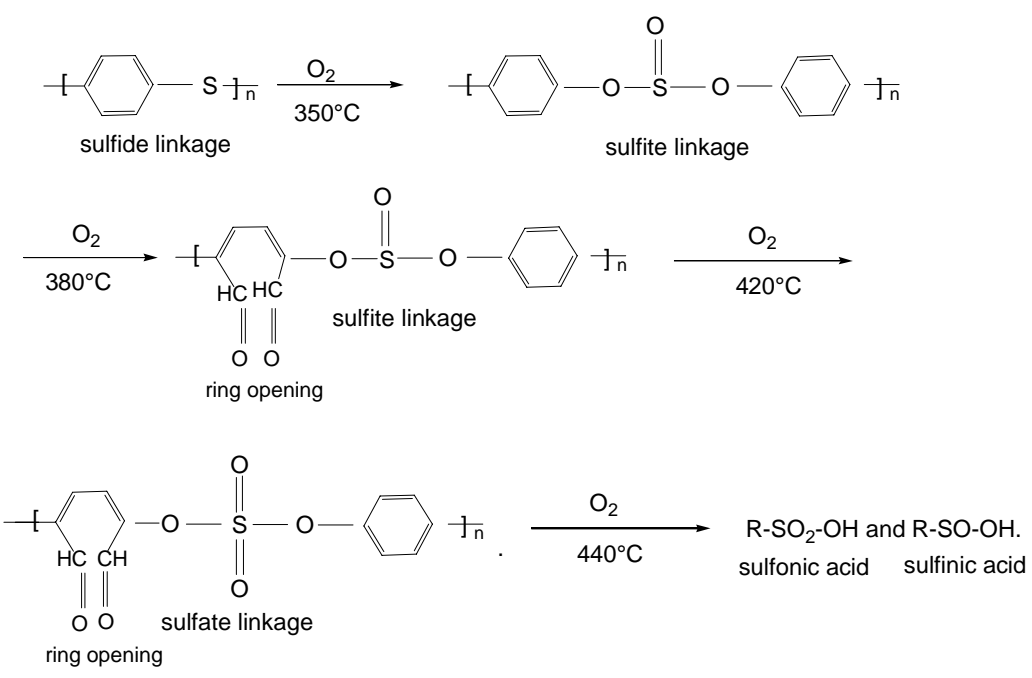

\section{Figure 41. Schematic representation of thermal oxidation pathway for PPS film in temperature range $350^{\circ}-440^{\circ} \mathrm{C}$.}

In trying to enhance the melting point of PPS, our particular interest was in adapting the polymer/clay nanocomposite technology by using montomorillonite (MMT) clay as the alternative nanoscale filler. As well documented by many investigators [4247], MMT consists of one alumina octahedral sheet sandwiched between two silica tetrahedral sheets in which the fundamental unit is a one nanometer (nm) thick, and has an approximately one hundred to several hundred nm long planar structure. Importantly, the basal space and gallery of $\sim 1.1 \mathrm{~nm}$ between the alumina and silica sheets includes various cation-exchangeable $\mathrm{Na}^{+}, \mathrm{K}^{+}, \mathrm{Li}^{+}$, or $\mathrm{Ca}^{2+}$ ions. These ions can easily exchanging 
with organic alkyl amine cations. Thus, the initial step of preparing a nanoscale MMT filler is the intercalation of such organic macromolecule ion exchangers containing a long alkyl chain linked to ionic amine into the gallery, thereby substantially expanding the gallery. Then, these spaces in the gallery expanded by the intercalated alkyl amine macromolecules lead to promoting the co-intercalation of polymers during curing, followed by the exfoliation of individual silicate platelets of $\sim$ one nm thick. Accordingly, the aspect ratio of the exfoliated MMT nanofiller in the polymer matrix is very large. As a result, such dispersion of exfoliated MMT nanofillers significantly improved some properties of the polymer including mechanical properties, thermal stability, and fire retardation [48-51].

Based upon this information, the emphasis of the present study was directed towards investigating the usefulness of chemically treated MMT nanofillers in enhancing the melting point of PPS. Also, it included defining the characteristics of MMT-filled PPS nanocomposite material as a corrosion-preventing coating for carbon steel in a $\mathrm{CO}_{2}$ laden brine at $300^{\circ} \mathrm{C}$.

\subsubsection{Experimental}

Montomorillonite (MMT, 99 \% purity) filler was obtained from Aldrich Chemical Inc; it included cation-exchangeable sodium, $\mathrm{Na}$. The same company also provided the octadecylamine [ $\mathrm{CH}_{3}\left(\mathrm{CH}_{2}\right)_{17} \mathrm{NH}_{2}$, ODA, $90 \%$ purity] used as a pretreatment reagent for the MMT. The ODA was intercalated into the basal gallery of MMT in the following way. First, the two components, MMT dispersed in acidulated deionized water and the ODA dissolved in acidulated alcoholic solution, were separately prepared. For the first component, $10 \mathrm{~g}$ of MMT was added to $500 \mathrm{~g}$ acidulated deionized water containing $25 \mathrm{~g}$ of $1 \mathrm{~N} \mathrm{HCl}$, and then agitated for 10 hours at $75^{\circ} \mathrm{C}$. The resulting colloidal MMT suspension was left for 20 hours to cool to the room temperature, and then was filtered. The MMT particles retained by the filer were dried for 20 hours at $110^{\circ} \mathrm{C}$. Meanwhile, $2.5 \mathrm{~g}$ ODA was incorporated into a solution consisting of $50 \mathrm{~g}$ of isopropyl alcohol (IPA) and $31 \mathrm{~g}$ of $1 \mathrm{~N} \mathrm{HCl}$, and then continuously stirred until the ODA completely dissolved in this acidulated alcoholic solution, and the solution became transparent. Afterward, $50 \mathrm{~g}$ deionized water was added to the ODA solution. Next, $6.7 \mathrm{~g}$ of dried MMT (the first 
component) was added to the ODA solution (the second component), and the mix was vigorously agitated for 5 hours at room temperature to make slurry with suspended MMT particles. The suspension was filtered, and the solid particles remaining on the filter were repeatedly washed with deionized water to eliminate any extra ODA as far as possible, without removing intercalated ones, and then dried for 24 hours at $110^{\circ} \mathrm{C}$. The dried ODA-treated MMT mass was pulverized in a vibrating sample mill for use as the filler.

A thermoplastic polyphenylenesulfide (PPS, $99.9 \%$ purity) powder with a particle size of $<60 \mu \mathrm{m}$ was obtained from Ticona. A 45wt\% PPS powder was mixed with 55wt\% isopropyl alcohol (99.9 \% purity) to make a slurry coating. Then, MMT filler at 5, 8, 11, and $14 \%$ by weight of the total amount of PPS was added to the slurry, and the mix was mechanically blended in a shear blender for 2 min to uniformly disperse the MMT particles throughout the slurry. The MMT-filled PPS coating systems were deposited on the Zn.Ph-primed coupons as follows. The primed coupons were dipped into the slurry, and withdrawn slowly. The slurry -covered coupons were left for 20 hours at ambient temperatures to volatilize the isopropyl alcohol, and simultaneously, to promote the conversion of the slurry layer into a sintering layer. Then, the sintered layer was heated in air at $310^{\circ} \mathrm{C}$ for 3 hours to achieve melt flow, and subsequently cooled to room temperature to make a solid film. This coating process was repeated three times more to assemble coating films ranging from 120 to $150 \mu \mathrm{m}$ thick.

\subsubsection{Results}

The cation- exchange reaction between octadecylamine (ODA) and montomorillonite (MMT) clay led to the intercalation of ODA into the $\sim 1.1 \mathrm{~nm}$ gallery within the MMT structure. Further, this intercalation played an important role in promoting the exfoliation of individual nanoscale silicate plates formed by the cointercalation of molten polyphenylensulfide (PPS), thereby generating a nanoscale MMTfilled PPS nanocomposite. Exfoliation not only uniformly dispersed the MMT nanofillers in the PPS matrix (Figure 42), but also conferred three advanced properties on semicrystalline PPS polymer; 1) an increase in its melting temperature by nearly $40^{\circ}$ to $290^{\circ} \mathrm{C}$ (Figure 43), 2) enhanced crystallization energy, reflecting the development of a good interfacial bond between nanofiller and PPS (Figure 44), and, 3) abatement of its 
hydrothermal oxidation attributed to the transformation of sulfide $\rightarrow$ sulfite linkage. When this advanced PPS/MMT nanocomposite was used as the high temperature corrosion-preventing coating of ZnPh-primed carbon steel, a coating of $\sim 150 \mu \mathrm{m}$ thickness adequately mitigated corrosion of steel during an exposure for 20 days in a $300^{\circ} \mathrm{C}$ brine environment. In fact, there were no significant changes in the coating's pore resistance, Rp, that represents the extent of the uptake of corrosive ionic species (Figure 45). In contrast, the Rp value of the coating without MMT declined with increasing exposure time, meaning that prolonging the exposure time enhanced the extent of infiltration of corrosive ionic species through the coating layer. Also, the adherence of the exposed nanocomposite coating to the $\mathrm{Zn}$.Ph primer was outstanding, as verified by the fact that the bond failure at interfaces between the coating and primer took place in the primer layer.
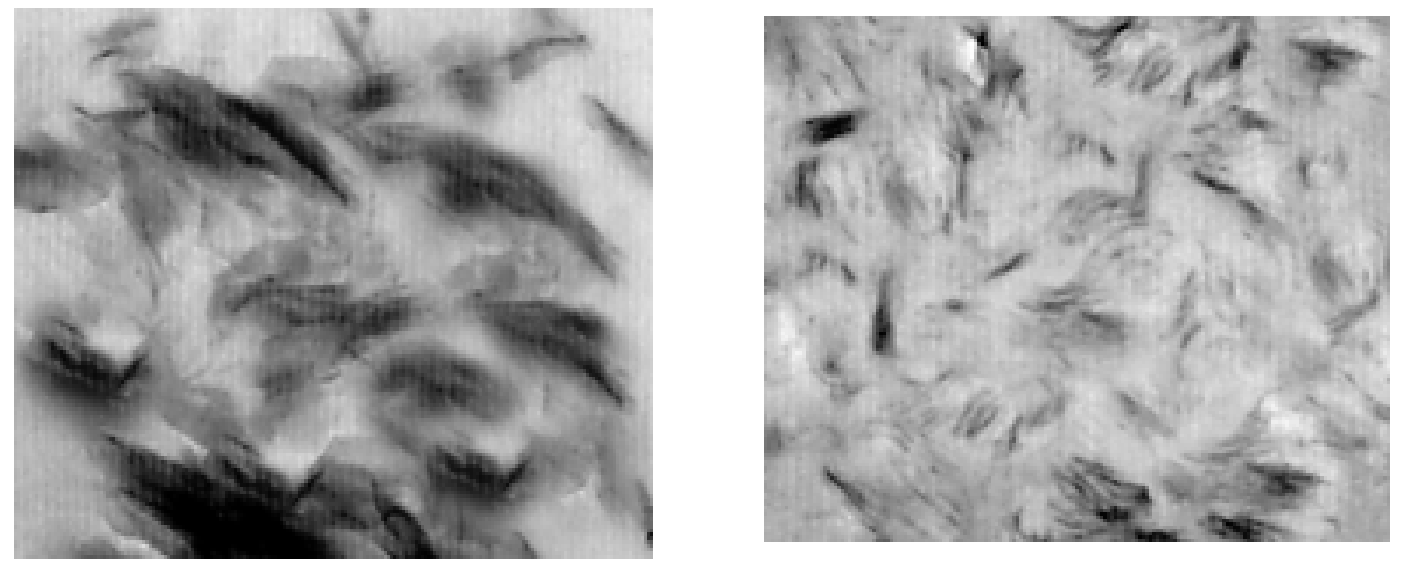

Figure 42. Transmission Electron Microscopy (TEM) images of untreated MMT/PPS (left) and ODA-treated MMT/PPS nanocomposite (right) systems. 


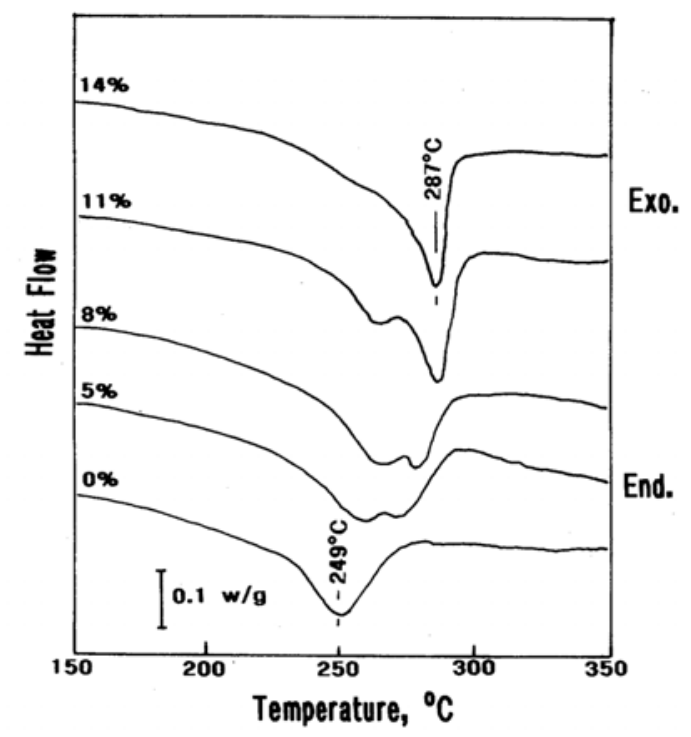

Figure 43. Shift in the melting temperature of PPS as a function of the content of treated MMT.

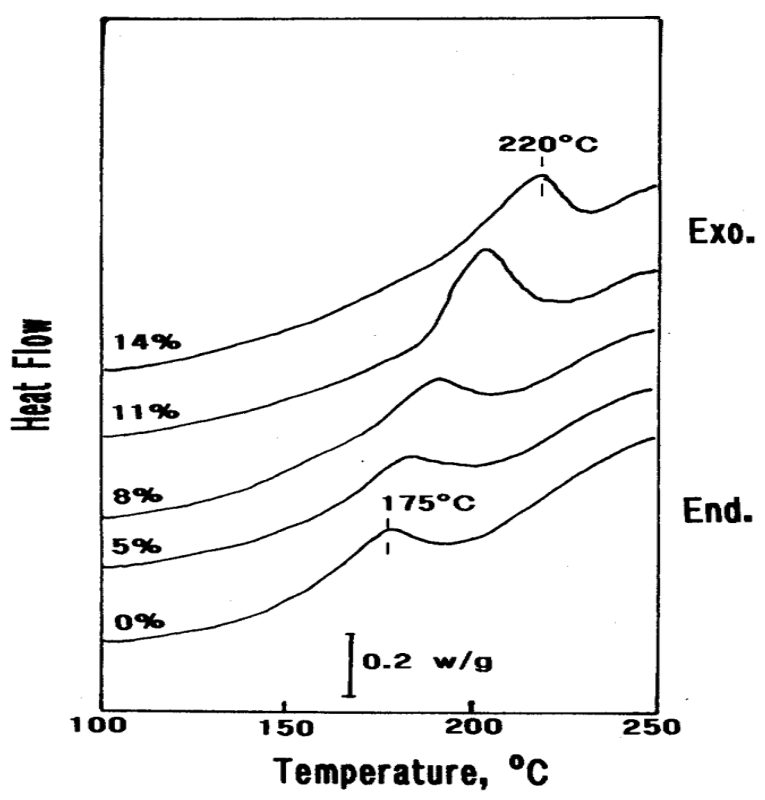

Figure 44. Changes in the exothermic crystallization temperature of PPS as a function of the content of treated MMT. 


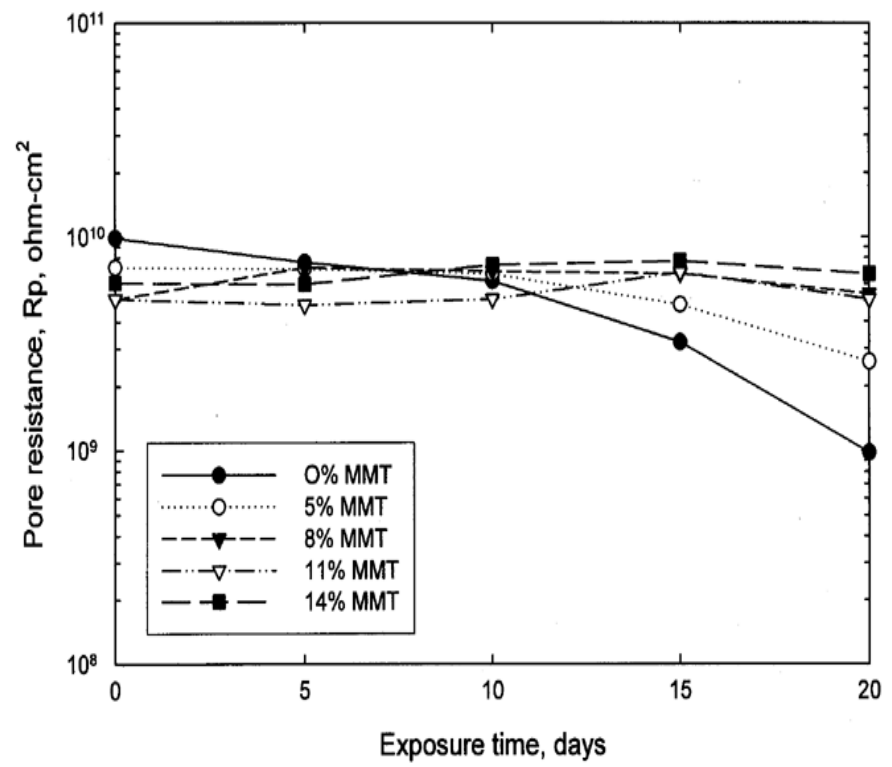

Figure 45. Pore reistance, Rp, of the PPS coatings containing 0, 5, 8, 11, and 14 wt\% treated MMT after exposure for up to 20 days in a $300^{\circ} \mathrm{C}$ brine.

\subsection{Field Exposure Test}

Based upon the information above, the 1.5-in.-diam. carbon steel elbow was coated with 14wt\% treated MMT-filled PPS nanocomposite material (Figure 46), and then the coated elbow was sent to CalEnergy power plant, CA, operating the $\mathrm{pH} \sim 2$, brine temperature of $\sim 260^{\circ} \mathrm{C}$ to conduct the field validation test. The reason for a low $\mathrm{pH}$ environment was due to the use of concentrated $\mathrm{HCl}$ as scale inhibitor. As is seen in a photo, all surfaces are coated with a 0.011-in.-thick multi-layer nanocompostite. The coating has been smoothed on the flange surfaces to allow the gaskets to seal. The field test of this test article is currently being undertaken.

The original assembly of this article was specified in zirconium, which costs about $\$ 150 / \mathrm{kg}$. Carbon steal is about $\$ 0.50 / \mathrm{kg}$, and PPS, $\$ 12 / \mathrm{kg}$. If success, with the amount of PPS applied and the dimensions of the assembly, a preliminary cost estimate shows that using PPS-coated carbon steel results in a $98 \%$ savings in material expense. 


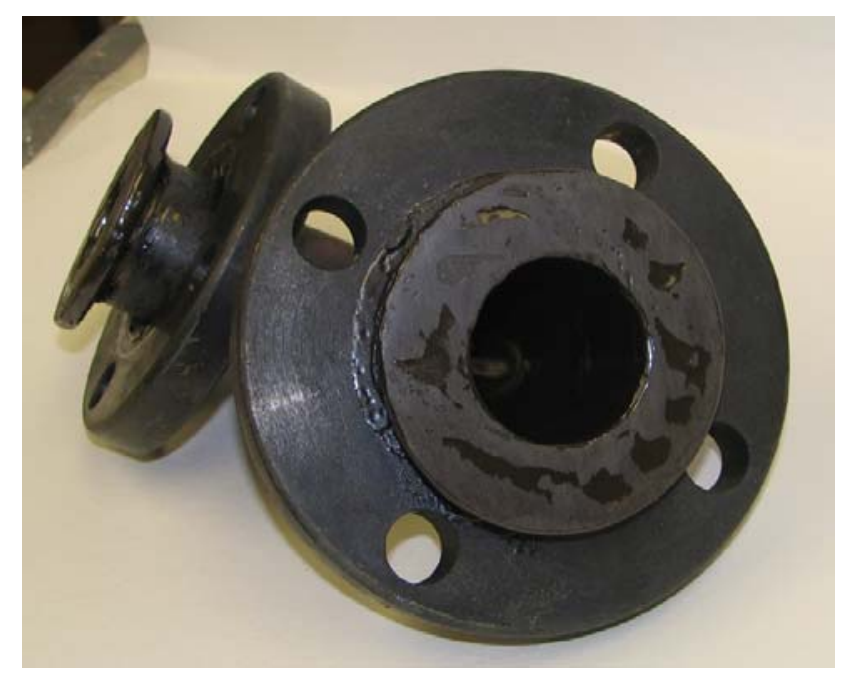

Figure 46. Carbon steel elbow coated with MMT-filled PPS nanocomposite being prepared for field exposure test at CalEnergy.

\subsection{Conclusions}

1. Titanium carbonitride $[(\mathrm{Ti}(\mathrm{CN})]$ coating was deposited onto steel surfaces by chemical vapor deposition (CVD) technology at a moderate temperature; then, its effectiveness in preventing the corrosion of steel was evaluated in an acid-brine geothermal environment at $200^{\circ} \mathrm{C}$ under a hydrothermal pressure of $1.45 \mathrm{MPa}$. Two undesirable attributes caused its failure as corrosion-preventing coatings: One factor was the susceptibility of $\mathrm{Ti}(\mathrm{CN})$ to oxidation reactions with aqueous steam, leading to the formation of $\mathrm{TiO}_{2}$ scale. Furthermore, this $\mathrm{TiO}_{2}$ scale then was transformed into water-soluble $\mathrm{TiCl}_{4}$ salt by the attack of acid brine, thereby resulting in its dissolution in the aqueous medium. The corrosion of $\mathrm{Ti}(\mathrm{CN})$ in such an environment took place through the processes of oxidation 6 chlorination 6 dissolution. The other factor was the inherent defects and pores in the coating layers, allowing the corrosive species to infiltrate easily. In fact, corrosion products from the underlying steel were observed at several different locations in the coating's surfaces. 
2. Although the flame-sprayed $\mathrm{Ni}-\mathrm{Al}$ coatings had an excellent thermal conductivity and a good wear-resistance, the inherent open structure of these coatings allowed the hot brine to permeate them easily under such pressure, causing the development of corrosion-induced stress cracks in the carbon steel. Furthermore, under $250^{\circ} \mathrm{C}$ brine environment, the coatings underwent oxidation with the formulation $\mathrm{Al}_{2} \mathrm{O}_{3}$ as the major scale compound and $\mathrm{NiO}$ as the minor one.

3. Nanoscale montomorillonite (MMT) clay fillers became dispersed in a polyphenylenesulfied (PPS) matrix through the processes of octadecylamine (ODA) intercalation $\rightarrow$ molten PPS co-intercalation $\rightarrow$ exfoliation. Cooling this molten exfoliated material led to the formation of a PPS/MMT nanocomposite. The MMT nanofiller conferred three advanced properties on the semi-crystalline PPS: First, it raised its melting point by nearly $40^{\circ} \mathrm{C}$ to $290^{\circ} \mathrm{C}$; second, it increased its crystallization energy, implying that an excellent adherence of the nanofillers' surfaces to PPS in terms of a good interfacial bond; and, third, it abated the degree of its hydrothermal oxidation due to sulfide $\rightarrow$ sulfite linkage transformations. When this advanced PPS nanocomposite was used as a corrosion-preventing coating for carbon steel in a simulated geothermal environment at $300^{\circ} \mathrm{C}$, a coating of $\sim 150 \mu \mathrm{m}$ thickness adequately protected the steel against hot brine-caused corrosion. In contrast, an MMT-free PPS coating of similar thickness was not nearly as effective in mitigating corrosion as was the nanocompsite; in fact, the uptake of corrosive ionic electrolyte by the unmodified coating increased with an extending exposure time.

\subsection{Publications}

- Sugama, T., "Polyphenylenesulfied/Montomorillonite Clay Nanocomposite Coatings: Their Efficacy in Protecting Steel Against Corrosion”, Matter Letters, (in press).

- Sugama, T. “CVD-Titanium Carbonitride Coatings as Corrosion-Preventing Barriers for Steel in Acid Brine at $200^{\circ}$ C”, Materials Letters, 38, 227-234 (1999). 
- Sugama, T. "Yttrium Acetate-Derived Particle Coatings for Mitigating Oxidation and Corrosion of Inconel 625", J. Sol-Gel Sci. Tech., 12, 35-48 (1998).

- Sugama, T. "Polyphenylene sulfide-Sealed Ni-Al Coatings for Protecting Steel from Corrosion and Oxidation in Geothermal Environments”, J. Materials Science, $\underline{33}$, 3791-3803 (1998).

- $\quad$ Sugama, T. " $\mathrm{Y}_{2} \mathrm{O}_{3}$-sealed Ni-Al Protective Coatings for Inconel 625", $\underline{\text { Surface }}$ Coat. Tech., 106, 106-116 (1998).

\section{Coatings for Condensers}

Regarding the feature of air-cooled aluminum-finned steel condenser tubes, the aluminum fins circling the surfaces of tube are of many different shapes and structures; some of them have zigzag- and wave-shaped conformations. Further, the distance between the fins is less than $3 \mathrm{~mm}$, while the height of fin attached to the steel tube is $15 \mathrm{~mm}$. Thus, the coating solutions to be used are required to be an environmentally benign water-based solution possessing the following characteristics; 1) a low surface tension allowing it to easily permeate and wick through between the fins, and, 2) good wetting behavior on the surfaces of both aluminum and steel. Correspondingly, once the coating solution wetting the surfaces of the entire condenser is converted into a solid film, the film must form a void-free uniform, continuous thin coating layer. Also, its surface must be hydrophobic, yet be inert chemically to silica and silicate scales. All these requirements are essential in fabricating an anti-corrosion and anti-fouling barrier layer to cover the condenser's entire surfaces.

Our previous study of water-based organometallic polymer (OMP) coatings for replacing the environmentally hazardous conventional $\mathrm{Cr}$ and $\mathrm{Pb}$ metal-containing coatings was directed toward synthesizing several metal-containing siloxane polymers by a sol-gel technology that involves a hydrolysis-condensation reaction between the colloidal precursors containing various different organosilanes and the metal alkoxides. The synthesized OMPs then were evaluated to obtain information on their usefulness as corrosion-preventing coatings for lightweight metals, such as aluminum, zinc, and magnesium alloys [52]. Among the organosilane compounds, the 3aminopropyltrimethoxy-silane (APTMS) monomer was one of the effective organosilane precursors in ensuring that the assembled OMP coating adequately protects the metals 
against corrosion and satisfactorily substitutes for the environmentally unacceptable coatings. In polymerizing the APTMS monomer, mineral acid was used to expedite the rate of hydrolysis-condensation reactions of the trimethoxy-silane group, $\equiv \mathrm{Si}\left(-\mathrm{OCH}_{3}\right)_{3}$, within the APTMS; $\equiv \mathrm{Si}\left(\mathrm{OCH}_{3}\right)_{3} \rightarrow \equiv \mathrm{Si}(\mathrm{OH})_{3} \rightarrow \equiv(\mathrm{Si}-\mathrm{O}-\mathrm{Si})_{\mathrm{n}} \equiv$.

Based upon the background described above, our objective in this work centered on assessing the ability of a new-type aminopropylsilane triol (APST) precursor made by substituting the trimethoxy group, $\left(\mathrm{OCH}_{3}\right)_{3}$, within the APTMS for the triol group, $(\mathrm{OH})_{3}$, to protect the air-cooled condensers against corrosion. This work also involved the modification of APST with the cerium (Ce) acetate. Most of our attention was focused on the corrosion rather than studying scale deposition and its prevention. The factors to be assessed included the changes in the extent of wettability and spreadability of the unmodified and modified APST precursors over the both aluminum and carbon steel substrates as a function of its surface tension, the hydrophilic and hydrophobic properties of unmodified and modified APST solid film's surfaces, the thermal stability of film, the precursor $\rightarrow$ polymer conversion mechanism, and the adherence of films to aluminum and steel substrates. All the data were integrated and correlated directly with information obtained from the corrosion-related studies.

\subsection{Polyaminopropylsiloxane Coatings Derived from Unmodified APST}

\subsubsection{Experimental}

Aminopropylsilane triol (APST) (25\% in water), supplied by Gelest, Inc., was used as a network-forming monomeric organosilane precursor. A 25\% APST was diluted by adding an appropriate amount of deionized water to prepare a 2, 5, 10, and $20 \%$ APST aqueous precursor solution. Some $\mathrm{HCl}$ then was incorporated into these precursor solutions in an attempt to adjust the $\mathrm{pH}$ level to within the range of 10.1 to 10.5. The metal substrates were 6061-T6 aluminum (Al) sheet and AISI 1008 carbon steel.

These substrate surfaces were coated with the APST precursor solutions in the following sequence. First, to remove any surface contaminants, the substrates were immersed for $20 \mathrm{~min}$ at $80^{\circ} \mathrm{C}$ in an alkaline solution consisting of $0.4 \mathrm{wt} \% \mathrm{NaOH}, 2.8$ wt $\%$ tetrasodium pyrophosphate, $2.8 \mathrm{wt} \%$ sodium bicarbonate, and $94.0 \mathrm{wt} \%$ water. The alkali-cleaned substrates were washed with deionized water at $25^{\circ} \mathrm{C}$ for $1 \mathrm{~min}$, and dried 
for $15 \mathrm{~min}$ at $100^{\circ} \mathrm{C}$. Then, the substrates were dipped into a soaking bath of precursor solutions at room temperature, and withdrawn slowly. The wetted substrates were heated in an oven for $120 \mathrm{~min}$ at a temperature of $100^{\circ}, 145^{\circ}, 175^{\circ}$, and $200^{\circ} \mathrm{C}$, to yield thin solid films. This dip-withdrawing-baking coating process was repeated once more to deposit dual films on the metal's surface. Using the Dektak Surface Profile Measurement System, the thickness of the solid films deposited on the substrates from 2, 5, 10, and 20 wt $\%$ APST solutions were $~ 0.3, \sim 0.7, \sim 1.2$, and $\sim 2.2 \mu \mathrm{m}$, respectively.

\subsubsection{Results}

Specific amounts of APST in this precursor solution correspondingly reduced the surface tension of the water. For instance, the surface tension of water declined from 73.0 dynes/cm to 50.9 dynes/cm when $10 \mathrm{wt} \%$ APST was added to the water. Since the aircooled condenser is composed of two metal components, aluminum fins and carbon steel tube, the precursors must be compatible with both metals. The magnitude of the wetting of the metals depended on the surface tension of the precursors; namely, a low surface tension enhanced wetting performance. Although both metals were cleaned with the same hot alkali solution, the surfaces of aluminum possessed better wetting characteristics compared with those of the steel component (Figure 47). The precursor solution was transformed into a solid film by heating it, which led to condensation reactions between the silanol groups within the APST, thereby forming the network structure of the polyaminopropylsiloxane (PAPS) polymer;

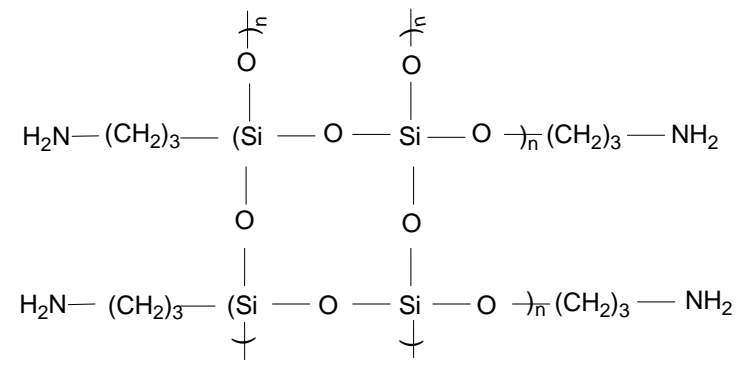




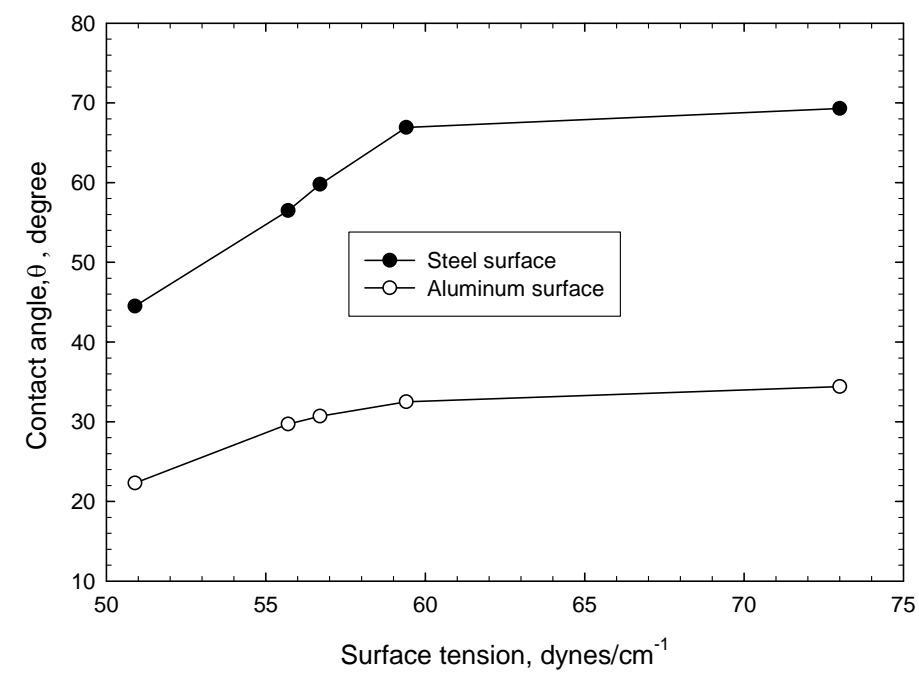

Figure 47. Relation between surface tension and contact angle for APST solutions.

We found that $175^{\circ} \mathrm{C}$ was the most effective heating temperature in making a PAPS film less susceptible to moisture. Further, a thin PAPS coating film $(2.2 \mu \mathrm{m})$ adhered to both these metals, but bonded more favorably to the aluminum's surfaces rather than to those of the steel. These two important properties, excellent wetting by the APST precursor and good adherent to the PAPS film, played an essential role in greatly protecting the aluminum against corrosion. In fact, the corrosion rate, milli-inches per year (mpy) of the bare aluminum was considerably reduced from 1.601 mpy to $1.875 \mathrm{x}$ $10^{-3}$ mpy by depositing on it a $10 \mathrm{wt} \%$ APST-derived PAPS film of $\sim 1.2 \mu \mathrm{m}$ thick. In contrast, using the same APST concentration, the effectiveness of PAPS film in reducing the corrosion rate of steel was much less, by nearly two orders of magnitude. These results were directly correlated with the salt-spraying resistance of the coated metals. By coating bare aluminum with $20 \mathrm{wt} \%$ APST-derived PAPS, its useful lifetime in a $5 \%$ salt-fog chamber at $35^{\circ} \mathrm{C}$ was extended from only $~ 40$ hours to more than 1400 hours. By comparison, this coating extended the useful lifespan of steel from $\sim 10$ hours to 216 hours (Table 6). 
Table 6. Salt-spray resistance tests for non-coated and coated steel and Al panels

\begin{tabular}{|c|c|c|}
\hline Substrate & APST, wt \% & $\begin{array}{c}\text { Salt-spray resistance, } \\
\text { hours }\end{array}$ \\
\hline Steel & 0 & $\sim 10$ \\
\hline Steel & 2 & $\sim 30$ \\
\hline Steel & 5 & $\sim 48$ \\
\hline Steel & 10 & $\sim 120$ \\
\hline Steel & 20 & $\sim 216$ \\
\hline $\mathrm{Al}$ & 0 & $\sim 40$ \\
\hline $\mathrm{Al}$ & 2 & $\sim 240$ \\
\hline $\mathrm{Al}$ & 5 & $\sim 984$ \\
\hline $\mathrm{Al}$ & 10 & $\sim 1224$ \\
\hline $\mathrm{Al}$ & 20 & $>1400$ \\
\hline
\end{tabular}

In trying to further visualize and corroborate the information described above, the cut sections of two condensers with different shape of aluminum fins were coated with 5 wt $\%$ and $20 \mathrm{wt} \%$ APST-derived PAPS polymers and exposed to salt-spraying chamber for 1440 hours. Figure 48 gave the photographs of the appearance of these exposed condensers. The surfaces of PAPS coating are represented by a light golden color. The aluminum fins of the condenser coated with a $5 \mathrm{wt} \%$ APST film were locally corroded, and some corrosion was visually observed in the internal carbon tube. In contrast, although some rust stain was found in the tube, using a $20 \mathrm{wt} \%$ APST film significantly reduced the rate of corrosion of the aluminum fins. This finding strongly validated that the useful lifetime of air-cooled condensers is extended conspicuously using an appropriate amount of APST precursor. 


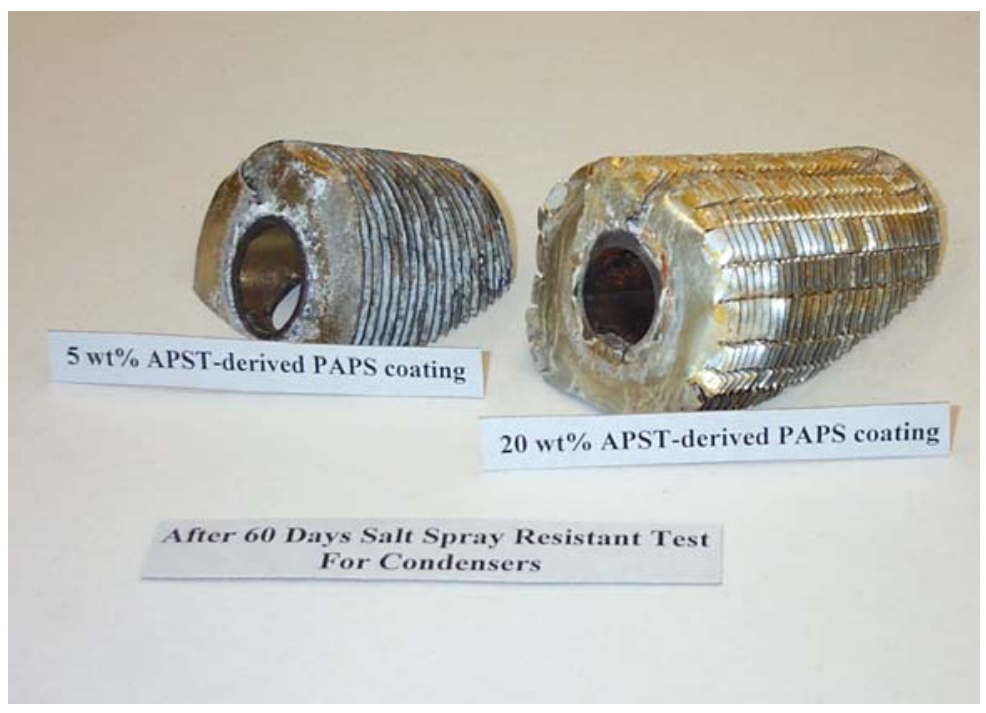

Figure 48. Appearance of the condensers coated with 5 wt $\%$ and 20 wt $\%$ APSTderived PAPS after a 60-day salt spray resistant test.

\subsection{Ce Oxide/Poly-acetamide-acetoxyl methyl-propylsiloxane (PAAMPS) Nanocomposite Coatings Derived from Ce acetate-modified APST}

Emphasis in this work was directed toward improving the corrosion-preventing performance of previously developed PAPS coating for the condensers. Ideally, not only would the improved coating adequately protect the steel tubes in the condensers against corrosion, but also its thin film would mitigate the corrosion of the aluminum fins. Several recent papers [53-58] documented that, among the rare-earth metal salts, the environmentally benign cerium (Ce) salt compounds displayed high potential as a promising alternative corrosion inhibitor for replacing the toxic hexavalent chromium $\left(\mathrm{Cr}^{6+}\right)$ salt compounds used as a pigment in the polymer coatings and conversion coatings in anodizing baths. The formation of Ce oxide- and hydroxide-based passive layers over the aluminum and steel surfaces was thought to be the major contributor to mitigating the corrosion of these metals.

Accordingly, our particular interest was to incorporate this green Ce-based salt compound as a corrosion-inhibiting dopant into the APST and to investigate its ability to enhance the potential of a polysiloxane-based polymer as a corrosion-preventing thin 
barrier layer. From among the Ce-based salt compounds, Ce acetate was chosen for this study. Our study covered the changes in the extent of wettability and spreadability of the APST precursor solutions over both aluminum and steel substrates as a function of the concentration of Ce acetate dopant, the susceptibility to moisture of the surfaces of the polymer film derived from Ce acetate-dopted APST, the conformation of the polymers yielded by the reaction between the Ce acetate and APST, the thermal stability of these polymers, the phase identification of Ce oxides, and the chemistry at the outermost surface sites of the coating films. All the data were integrated and correlated directly with information obtained from the corrosion-related studies including the potentiodynamic polarization curve and salt-spraying resistance.

\subsubsection{Experimental}

Aminopropylsilane triol $\left[\mathrm{H}_{2} \mathrm{~N}-\left(\mathrm{CH}_{2}\right)_{3}-\mathrm{Si}(\mathrm{OH})_{3}\right.$, APST] (25\% in water), supplied by Gelest, Inc., was used as a network-forming monomeric organosilane precursor. A 25\% APST solution was diluted by adding an appropriate amount of deionized water to prepare a $20 \%$ APST aqueous precursor. The cerium (Ce, III) acetate hydrate, $\left(\mathrm{CH}_{3} \mathrm{CO}_{2}\right)_{3} \mathrm{Ce} \cdot \mathrm{xH}_{2} \mathrm{O}$, as the corrosion-inhibiting dopant in APST, was obtained from Aldrich. The $0.2,0.5,1.0,2.0,3.0,6.0$, and $10.0 \mathrm{wt} \%$ Ce acetate was added to the 20 wt $\%$ APST solution to prepare the standard Ce acetate-doped APST solutions. To fabricate a thin film, the standard solutions then were further diluted with deionized water to make a $0.5,1.0$, and $3.0 \mathrm{wt} \%$ doped solutions. The metal substrates, supplied from Advanced Coating Technologies, Inc., were 3003 aluminum (Al) and AISI 1008 carbon steel test panels.

The surfaces of these substrates were coated with the non-doped and doped APST precursor solutions in the following sequence. First, to remove any surface contaminants, the test panels were immersed for $20 \mathrm{~min}$ at $80^{\circ} \mathrm{C}$ in an alkaline solution consisting of 0.4 wt $\% \mathrm{NaOH}, 2.8 \mathrm{wt} \%$ tetrasodium pyrophosphate, $2.8 \mathrm{wt} \%$ sodium bicarbonate, and 94.0 wt $\%$ water. The alkali-cleaned panles were washed with deionized water at $25^{\circ} \mathrm{C}$ for 1 $\min$, and dried for $15 \mathrm{~min}$ at $100^{\circ} \mathrm{C}$. Then, the panels were dipped into a soaking bath of precursor solutions at room temperature, and withdrawn slowly. The wetted substrates were heated in an oven for 120 min at $150^{\circ} \mathrm{C}$ to yield solid films. 


\subsubsection{Results}

The incorporation of Ce acetate not only reduced the $\mathrm{pH}$ of APST solution, ranging from 10.94 for $0 \mathrm{wt} \%$ Ce acetate to 9.49 for10wt\%, but also increased the surface tension of the APST solutions. The latter factor directly affected the changes in wettability and spreadability of the modified solutions on aluminum and steel surfaces; namely, the high surface tension reduced wetting performance. Although both metals were cleaned with the same manner, the surfaces of aluminum possessed better wetting behaviors than those of steel. The transformation of the Ce acetate-doped APST solution into a solid film by heating it at $150^{\circ} \mathrm{C}$ was carried out through the following three spontaneous reactions: 1 ) the condensation reaction between the silanol, $\mathrm{SiOH}$, groups within the APST to assemble siloxane, Si-O-Si, linkages in the polypropylsiloxane polymer network, $\equiv \mathrm{Si}-\mathrm{OH}+\mathrm{HO}-\mathrm{Si} \equiv \rightarrow \equiv \mathrm{Si}-\mathrm{O}-\mathrm{Si} \equiv+\mathrm{H}_{2} \mathrm{O}$; 2) the amidating reaction between Ce acetate and amine, $\mathrm{NH}_{2}$, group in APST to form the acetamide moiety as the end reaction product in the polymer network, $\mathrm{Ce}\left(-\mathrm{O}-\mathrm{COCH}_{3}\right)_{3}+3 \mathrm{H}_{2} \mathrm{~N}-\rightarrow 3 \mathrm{CH}_{3} \mathrm{CO}-\mathrm{NH}-$ $+\mathrm{Ce}(\mathrm{OH})_{3}$; and, 3) the acetoxylation reaction between the Ce acetate and the silanol group to make the acetoxyl methyl moiety, $\mathrm{Ce}\left(-\mathrm{O}-\mathrm{COCH}_{3}\right)_{3}+3 \equiv \mathrm{Si}-\mathrm{OH} \rightarrow 3 \mathrm{CH}_{3} \mathrm{CO}-\mathrm{O}-$ $\mathrm{Si} \equiv+\mathrm{Ce}(\mathrm{OH})_{3}$. The last two reactions led to the in-situ conversion of Ce acetate into the Ce hydroxide, $\mathrm{Ce}(\mathrm{OH})_{3}$, derivative as the reaction byproduct, followed by its in-situ transformation into the nanoscale $\mathrm{Ce}^{3+}$ oxide, $\mathrm{Ce}_{2} \mathrm{O}_{3}$, during heating, $2 \mathrm{Ce}(\mathrm{OH})_{3} \rightarrow \mathrm{Ce}_{2} \mathrm{O}_{3}$ $+3 \mathrm{H}_{2} \mathrm{O}$. Most of amine groups were converted into the acetamide moiety when $3 \mathrm{wt} \% \mathrm{Ce}$ acetate was added to the APST. The extent of densification of siloxane linkages depended primarily on the content of Ce acetate; an increase in its content caused a low densification of siloxane linkage because of the depletion of silanol groups in the APST by an increased rate of acetoxylation. Nevertheless, the polymer structure formed through these reactions was categorized as the poly-acetamide-acetoxyl methyl-propylsiloxane (PAAMPS) polymer: 


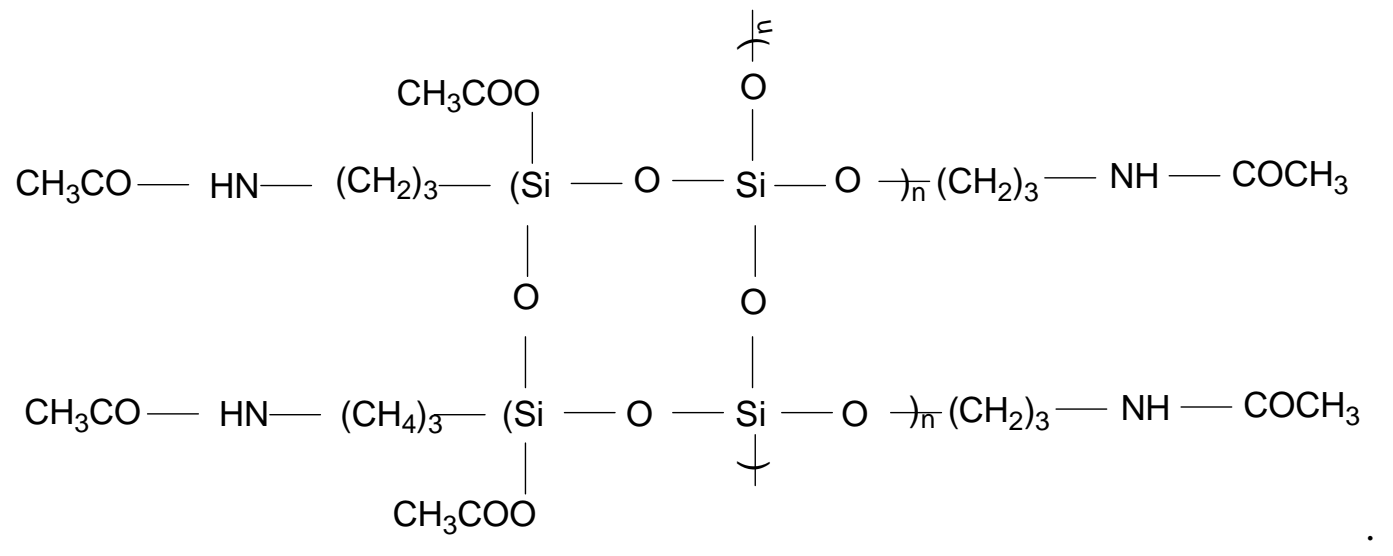

This new self-assembling nanocomposite synthetic technology allowed us to uniformly disperse the nanoscale Ce oxide particles in the PAAMPA matrix. We found that 3wt\% of Ce acetate was the best amount for conferring the maximum performance of a PAAMPS coating in mitigating the corrosion of both aluminum and steel. Among the factors governing this maximum performance was 1) a minimum content of non-reacted water-soluble APST and Ce acetate remaining in the coating, 2) a lower susceptibility of the coating's surfaces to moisture, thereby conferring water-repellent and-shedding properties, 3) the precipitation of a passive $\mathrm{Ce}^{3+}$ oxide film insensitive to $\mathrm{Cl}$ dissolution over the metal's surfaces, and, 4) excellent adherence of the coating to metal's surfaces. The combination of all these factors contributed to decreasing significantly the rate of cathodic oxygen reduction reactions at the corrosion site of metals, so extending their useful lifetime. With an $~ 8.5 \mu \mathrm{m}$ thick coating film, the useful lifetime of steel in a $5 \%$ salt-fog chamber at $35^{\circ} \mathrm{C}$ was prolonged from only $\sim 10$ hours to $~ 768$ hours (Table 7). This lifetime was more than six times as long as that of steel coated with an APST without Ce acetate, strongly demonstrating the great effectiveness of Ce acetate in improving the ability of APST to protect steel against corrosion. The PAAMPS coating displayed a far better corrosion-preventing performance for aluminum, compared with that for steel. In fact, when a coating with nanoscale thickness $\leq 5 \mathrm{~nm}$ was deposited on the aluminum's surfaces, its corrosion rate, milli-inches per year (mpy) was nearly two orders of magnitude lower than that of bare aluminum (Table 8). In addition, the saltspray resistance of aluminum coated with this nanoscale film was strikingly extended from $\sim 40$ hours to $>1440$ hours. 
Table 7. Salt-spray resistance tests for uncoated and coated steel and aluminum panels.

\begin{tabular}{|l|l|l|}
\hline \multicolumn{1}{|c|}{ Substrate } & \multicolumn{1}{|c|}{ Ce acetate, wt\% } & \multicolumn{1}{c|}{$\begin{array}{c}\text { Salt-spray resistance, } \\
\text { hours }\end{array}$} \\
\hline Bare steel & - & $\sim 10$ \\
\hline Steel & 0 & $\sim 120$ \\
\hline Steel & 0.2 & $\sim 165$ \\
\hline Steel & 0.5 & $\sim 264$ \\
\hline Steel & 1.0 & $\sim 312$ \\
\hline Steel & 2.0 & $\sim 720$ \\
\hline Steel & 3.0 & $\sim 768$ \\
\hline Steel & 6.0 & $\sim 600$ \\
\hline Steel & 10.0 & $\sim 408$ \\
\hline Bare Aluminum & - & $\sim 40$ \\
\hline Aluminum & 0 & $\sim 980$ \\
\hline Aluminum & 0.2 & $\sim 1248$ \\
\hline Aluminum & 0.5 & $\sim 1400$ \\
\hline Aluminum & 1.0 & $>1800$ \\
\hline Aluminum & 2.0 & $>1800$ \\
\hline Aluminum & 3.0 & $>1800$ \\
\hline
\end{tabular}

Table 8. Tafel analyses for potentiodynamic polarization curves of thin films derived from 0.5, 1.0, and 3.0wt \% (3wt\% Ce acetate-modified 20wt\% APST) solutions for aluminum panels.

\begin{tabular}{|l|l|l|l|l|l|l|}
\hline Substrate & $\begin{array}{l}\text { Ce acetate- } \\
\text { modified } \\
\text { APST, } \\
\text { wt\% }\end{array}$ & $\begin{array}{l}\mathbf{E}_{\text {corr }}(\mathbf{I}=\mathbf{0}), \\
\mathbf{( V )}\end{array}$ & $\begin{array}{l}\boldsymbol{\beta}_{\mathbf{a}} \\
\mathbf{( V / d e c a d e )}\end{array}$ & $\begin{array}{l}\boldsymbol{\beta}_{\mathbf{c}} \\
(\mathbf{V} / \mathbf{d e c a d e})\end{array}$ & $\begin{array}{l}\mathbf{I}_{\text {corr, }} \\
\left(\mathbf{A} / \mathbf{c m}^{2}\right)\end{array}$ & $\begin{array}{l}\text { Corrosion } \\
\text { rate, } \\
\text { (mpy) }^{*}\end{array}$ \\
\hline $\begin{array}{l}\text { Uncoated } \\
\text { aluminum }\end{array}$ & - & -0.5690 & 0.0903 & 0.3792 & $\begin{array}{l}2.497 \times \\
10^{-7}\end{array}$ & $\begin{array}{l}1.070 \mathrm{x} \\
10^{-1}\end{array}$ \\
\hline Aluminum & 0.5 & -0.5429 & 0.0275 & 0.1758 & $\begin{array}{l}2.295 \times \\
10^{-8}\end{array}$ & $\begin{array}{l}9.829 \mathrm{x} \\
10^{-3}\end{array}$ \\
\hline Aluminum & 1 & -0.5353 & 0.0358 & 0.2140 & $\begin{array}{l}6.979 \times \\
10^{-9}\end{array}$ & $\begin{array}{l}2.989 \mathrm{x} \\
10^{-3}\end{array}$ \\
\hline Aluminum & 3 & -0.4926 & 0.0619 & 0.2055 & $\begin{array}{l}2.810 \times \\
10^{-9}\end{array}$ & $\begin{array}{l}1.203 \mathrm{x} \\
10^{-3}\end{array}$ \\
\hline
\end{tabular}

mpy: milli-inches per year

\subsection{Field Exposure Tests}


In our previous field tests at Mammoth power plant in 2003 and 2004, we evaluated the ability of the polyaminopropylsiloxane (PAPS) polymer coating to protect the surfaces of air-cooled condenser components, aluminum fins and carbon steel tube, against corrosion, and also to minimize the deposition of brine-induced mineral scales, such as silica and calcium compounds, on their surfaces. In this test, the aluminum fins and carbon coupons were covered with PAPS films of four different thickness, $\sim 2 \mu \mathrm{m}, \sim$ $0.5 \mu \mathrm{m}, \sim 50 \mathrm{~nm}$, and $~ 10 \mathrm{~nm}$, and then they were exposed in the NREL-designed brine sprayer apparatus, installed in the maintenance building at the Mammoth power plant. This apparatus subjected the coatings to a 15 minutes brine wet/dry cycle consisting of one-minute spray of cooled brine followed by 14 minutes of drying. The test results revealed that under such a hostile environment the PAPS coating film must be more than $2 \mu \mathrm{m}$ thick, and also was required to contain corrosion-inhibiting additives, such as rare earth metal oxides to further improve its corrosion- and fouling-preventing performance on both the aluminum fins and carbon steel pipes.

Based upon the information described above, our emphasis next shifted to evaluating the efficacy of PAAMPS/Ce oxide nanocomposite coating in protecting the aluminum fins and carbons steel coupons against corrosion in the wet/dry brine spray apparatus at the Mammoth. In this field test, the thickness of coating ranged from $3.5 \mu \mathrm{m}$ to $10 \mu \mathrm{m}$. For the comparison purpose, the Ce oxide-free PAPS coating was used in this test.

\subsubsection{Experimental}

Aminopropylsilane triol $\left[\mathrm{H}_{2} \mathrm{~N}-\left(\mathrm{CH}_{2}\right)_{3}-\mathrm{Si}(\mathrm{OH})_{3}\right.$, APST] (25wt \% in water), supplied by Gelest, Inc., was used as a network-forming monomeric organosilane precursor. The cerium (Ce, III) acetate hydrate, $\left(\mathrm{CH}_{3} \mathrm{CO}_{2}\right)_{3} \mathrm{Ce} \cdot \mathrm{xH}_{2} \mathrm{O}$, as the corrosion-inhibiting dopant in the APST, was obtained from Aldrich. To prepare the Ce acetate-doped APST precursor solutions, 3.0wt\% Ce acetate was added to the $25 \mathrm{wt} \%$ APST solution. The metal substrates were aluminum fins, and AISI 1008 carbon steel test panels.

The surfaces of these substrates were coated with the non-doped and doped APST precursor solutions in the following sequence. First, to remove any surface contaminants, the substrates were immersed for $20 \mathrm{~min}$ at $80^{\circ} \mathrm{C}$ in an alkaline solution consisting of 0.4 
wt\% $\mathrm{NaOH}, 2.8 \mathrm{wt} \%$ tetrasodium pyrophosphate, $2.8 \mathrm{wt} \%$ sodium bicarbonate, and 94.0 wt $\%$ water. The alkali-cleaned panels were washed with deionized water at $25^{\circ} \mathrm{C}$ for 1 min, and dried for $15 \mathrm{~min}$ at $100^{\circ} \mathrm{C}$. Then, the substrates were dipped into a soaking bath of precursor solutions at room temperature, and withdrawn slowly. The wetted substrates were left for 20 hours in an atmospheric environment at room temperature to convert the precursor solution into a gelled film. All the gelled films then were baked in an air oven for $120 \mathrm{~min}$ at $200^{\circ} \mathrm{C}$ to solidify them. The coated aluminum fins and carbon steel coupons then were exposed in the brine spray apparatus at the Mammoth to undergo an extremely harsh 24,500 brine wet/dry cycle fatigue test by NREL.

\subsubsection{Results}

Under an extremely harsh environment, exemplified by the 24,500 brine wet/dry cycle fatigue test, the Ce oxide/PAAMPS nanocomposite coating, fabricated by BNLdeveloped self-assembling nanocomposite technology, displayed very promising results in protecting two metal components, the aluminum fin (Figure 49) and carbon steel (Figure 50), of air-cooled condensers against corrosion, and in minimizing the deposition of scales. The uncoated aluminum fin and carbon steel underwent severe corrosion as reflected by the complete disintegration of fins and the accumulation and spallation of iron oxide-based corrosion scales on the carbon steel coupons. However, two critical issues of a further improvement of corrosion-preventing performance of this coating were considered: One was the poor coverage of the coating over the sharp edges of ring-shaped fin; we observed that most of the corrosion began at the fin's edge. The other issue was to develop more effective nanoscale rare earth metal oxides in inhibiting the cathodic corrosion reaction of metal surfaces underneath the coating, instead of using Ce oxide. Regarding the latter issue, we noted that the Ce oxide nanoparticles in contact with the underlying fin's surface formed an interfacial passive film coexisting with the polymer matrix. This passive film acted to retard the cathodic oxygen reduction reaction, $2 \mathrm{H}_{2} \mathrm{O}+$ $\mathrm{O}_{2}+4 \mathrm{e}^{-} \rightarrow 4 \mathrm{OH}^{-}$, at the corrosion site of the aluminum fin. However, although the Ce oxide offered an improved performance of the coating in inhibiting this reaction, the high-resolution SRFT-IR analysis revealed that the corrosion product, such as $\mathrm{Al}(\mathrm{OH})_{3}$, on the fin was generated at the interfaces between the fin and coating of $\sim 10 \mu \mathrm{m}$ 
thickness (Figure 51). This finding strongly suggested that $\sim 10 \mu \mathrm{m}$ thickness of coating was not enough to abate the permeation of corrosive electrolytes through it during this cycle testing; therefore, an effort must be made to seek other earth metal oxides that will further prevent the cathodic corrosion reaction at the fin/coating interfaces.

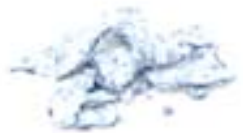

Uncoated

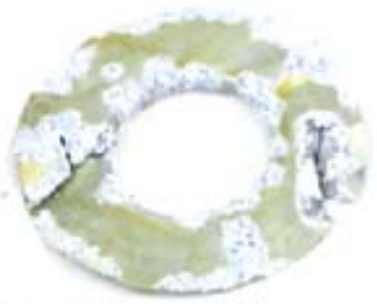

PAPS, $3.5 \mu \mathrm{m}$ thickness

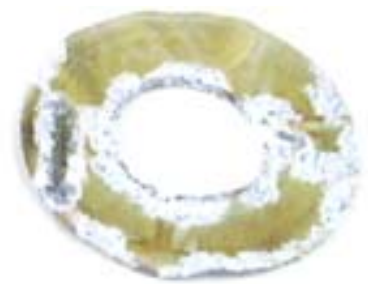

Ce oxide/PAAMPS, $3.5 \mu \mathrm{m}$ thickness

Figure 49. Appearance of uncoated, and PAPS- and Ce oxide/PAAMPS-coated aluminum fins after exposure in a $\mathbf{2 4 , 5 0 0}$ brine wet/dry cycle fatigue test.

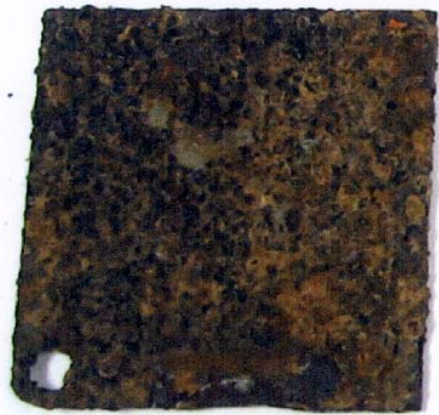

Uncoated

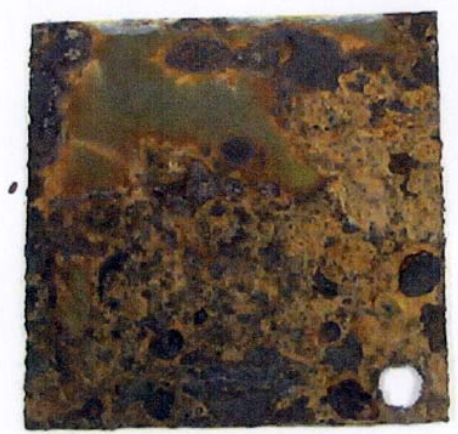

PAPS, $3.5 \mu \mathrm{m}$ thickness

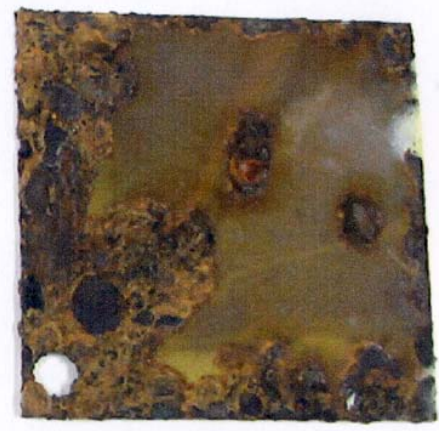

Ce oxide/PAAMPS, $3.5 \mu \mathrm{m}$ thickness

Figure 50. Appearance of uncoated, and PAPS- and Ce oxide/PAAMPS-coated carbon steel panels after a 24,500 cycle test. 


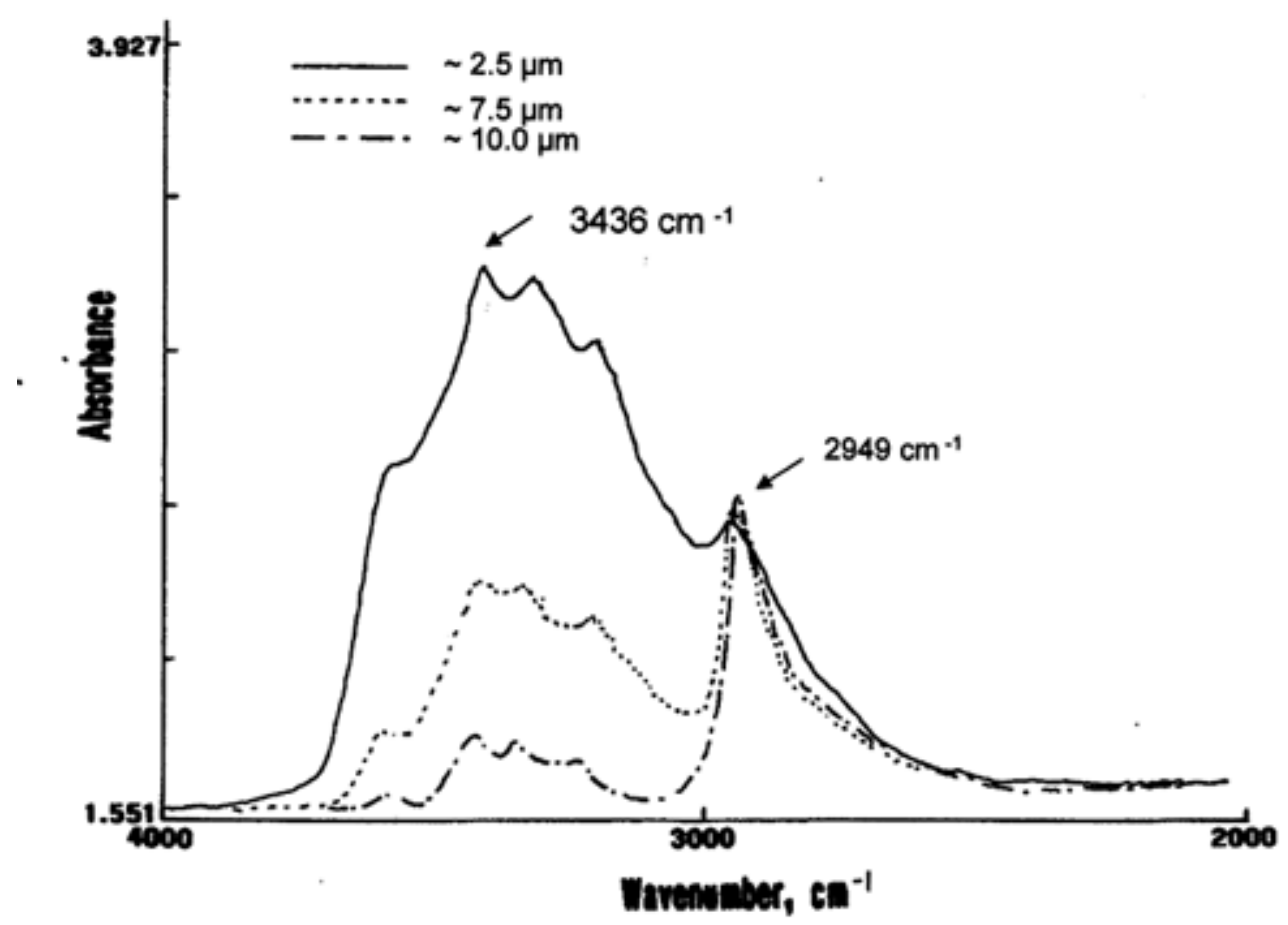

Figure 51. Comparison of peak intensities of $\mathrm{Al}(\mathrm{OH})_{3}$ - and $-\mathrm{CH}_{2}$ - related bands at 3436 and $2949 \mathrm{~cm}^{-1}$, respectively, for fins coated with Ce oxide/PAAMPS of $\sim 2.5$ $\mu \mathrm{m}, \sim 7.5 \mu \mathrm{m}$, and $\sim 10.0 \mu \mathrm{m}$ thickness after the cycle test.

\subsection{Conclusions}

1. A self-assembly nanocomposite synthesis technology was used to put together a $\mathrm{Ce}(\mathrm{OH})_{3}$ - dispersed poly-acetamide-acetoxyl methyl-propylsiloxane (PAAMPA) organometallic polymer. Three spontaneous reactions were involved, condensation, amidation, and acetoxylation, between the Ce acetate and aminopropylsilane triol (APST) at $150^{\circ} \mathrm{C}$. An increase in temperature to $200^{\circ} \mathrm{C}$ led to the in-situ phase transformation of $\mathrm{Ce}(\mathrm{OH})_{3}$ into $\mathrm{Ce}_{2} \mathrm{O}_{3}$ in the PAAMPA matrix. A further increase to $250^{\circ} \mathrm{C}$ caused oxidative degradation of the PAAMPA, thereby generating copious fissures in the composite. We assessed the potential of $\mathrm{Ce}(\mathrm{OH})_{3} /$ and $\mathrm{Ce}_{2} \mathrm{O}_{3} /$ PAAMPA composite materials as corrosionpreventing coatings for carbon steel and aluminum. The $\mathrm{Ce}_{2} \mathrm{O}_{3}$ composite coating displayed better performance in protecting both metals against $\mathrm{NaCl}$-caused corrosion than did the $\mathrm{Ce}(\mathrm{OH})_{3}$ composite. Using this coating formed at $200^{\circ} \mathrm{C}$, we demonstrated that the following four factors played an essential role in further mitigating the corrosion of the metals: First was a minimum susceptibility of 
coating's surface to moisture; second was an enhanced densification of the coating layer; third was the retardation of the cathodic oxygen reduction reaction at the metal's corrosion sites due to the deposition of $\mathrm{Ce}_{2} \mathrm{O}_{3}$ as a passive film over the metal's surface; and, fourth was its good adherence to metals. The last two factors contributed to minimizing the cathodic delamination of coating film from the metal's surface. We also noted that the affinity of the composite with the surface of aluminum was much stronger than that with steel. Correspondingly, the rate of corrosion of aluminum was reduced as much as two orders of magnitude by a nanoscale thick coating. In contrast, its ability to reduce the corrosion rate of steel was lower than one order of magnitude.

2. Under an extremely harsh environment, exemplified by the 24,500 brine wet/dry cycle field fatigue test at Mammoth power plant, this Ce oxide/PAAMPS nanocomposite coatings displayed very promising results in protecting two metal components, the aluminum fin and carbon steel, of air-cooled condensers against corrosion, and in minimizing the deposition of scales. However, two critical issues of a further improvement of corrosion-preventing performance of this coating were considered: One was the poor coverage of the coating over the sharp edges of ring-shaped fin; the other issue was to develop more effective nanoscale rare earth metal oxides in inhibiting the cathodic corrosion reaction of metal surfaces underneath the coating, instead of using Ce oxide.

\subsection{Patent}

Patent entitled “Self-assembly nanocomposite coatings” BNL Docket No. 369208 was filed, and this patent application was made in May 2005.

\subsection{Publications}

- Sugama, T., “Self-assembly Ce Oxide/Organopolysiloxane Composite Coatings”, J. Mater. Sci., (submitted).

- Sugama, T., "Cerium Acetate-modified Aminopropylsilane Triol: A Precursor of Corrosion-preventing Coating for Aluminum-finned Condensers”, J. Coat. Tech., 2, 649-659 (2005). 
- Sugama, T., Gawlik, K. and Jung, D., "Polyaminopropylsiloxane Coatings for Geothermal Air-cooled Condensers”, Recent Res. Devel. Mat. Sci., 4, 695-710 (2003).

\subsection{Conf. Proceeding}

- Gawlik, K; Sugama, T.; Jung, Doug. "Organometallic polymer coatings for geothermal-fluid-sprayed air-cooled condensers.” Geothermal Resources Council Transactions, 26, 657-661 (2002).

\section{Coatings for Steam Separators}

In our previous study [59], we evaluated the usefulness of polytetrafluoroethylene (PTFE) as the anti-oxidant polymeric additive to polyphenylenesulfide (PPS) coating at a brine temperature of $200^{\circ} \mathrm{C}$. The results revealed that the PTFE not only abated the hydrothermal oxidation of PPS, but also made the surface of the coating less susceptible to moisture. The principal reason for such contribution was due to the phase segregation of PTFE from PPS in the melt-flowing process of these mixed polymers at $320^{\circ} \mathrm{C}$; the segregated PTFE favorably migrated toward the outermost surface site of coating. Hence, the PTFE occupying the superficial layer of the coating played a very important role in creating two advanced properties; one was the improved hydrophobic surfaces offering an outstanding water repellency, and the other was a minimum uptake of oxygen by the coating's surfaces, reflected in its inertness to reactions with scales. However, one drawback of PTFE was that its chemical inertness caused poor adherence to the organic polymer and metal substrates. This fact meant that blending PTFE impaired the outstanding adherence of PPS to these substrates. Thus, the PPS/PTFE blend polymer was only used in the top layer of coating deposited on the underlying bulk PPS layer adhering to the primed metals. allowing it to scour off easily from liner's surfaces using hydroblasting cleaning device at low-pressure. Additionally, as is well known, the hydrophobic surfaces of PTFE had the lowest coefficient of friction corresponding to the lowest surface free energy among the conventional polymers [60]. Since the slippery surface of polymer contributes to lowering of its frictional resistance, PTFE's surfaces appear to possess good slipperiness, suggesting the potential use of this blend polymer as a coating for steam separators. Since the continuous operating temperature of the steam separator is $\sim 210^{\circ} \mathrm{C}$, this coating would be required to possess hydrothermal stability at 
least of $250^{\circ} \mathrm{C}$. So, if this coating withstands $300^{\circ} \mathrm{C}$ brine, it would guarantee that it would satisfactorily extend the useful lifetime of the carbons steel steam separators at $210^{\circ} \mathrm{C}$.

Based upon the above information, emphasis in the current work was directed towards assessing the ability of this blend polymer system prepared by varying the ratio of PTFE to PPS to protect the carbon steel against corrosion in a $\mathrm{CO}_{2}$-laden brine at $300^{\circ} \mathrm{C}$. Also, we investigated the alterations in the chemical state of unblended PTFE due to hydrothermal oxidation after exposure to hot brine. To obtain this information, this study had the following two major objectives: One was assessing the changes in the surface energy, the degree of oxidation, and the coefficient of friction of the coatings as a function of exposure time to estimate the extent of slickness and water repellency; the other was measuring thermal decomposition and the alterations of molecular structure to investigate the coating's hydrothermal stability. All the information then was integrated and correlated directly with the corrosion-related data obtained from AC electrochemical impedance spectroscopy (EIS).

\subsection{Experimental}

The polytetrafluoroethylene (PTFE) powder under the commercial trade name “SST-3H” supplied by Shammrock Technologies was used as a slip-enhancing and antioxidant polymeric additive to polyphenylenesulfide (PPS); it has a particle size of $\sim 40$ $\mu \mathrm{m}$. The PPS powder with a particle size of $<20 \mu \mathrm{m}$ was supplied by Ticone. PTFEblended PPS powder, with a PPS/PTFE ratio of 90/10, 80/20, 70/30, and 60/40 by weight, were prepared in a rotary blender. The AISI 1008 carbon steel panels (62.5 mm x $62.5 \mathrm{~mm}$ ) were used as the substrate.

As mentioned early, the PPS/PTFE blend polymer was only used as a top coating layer because of a poor adherence of PTFE to organic, inorganic, and metallic substrates. Accordingly, the coating systems deposited on the ZnPh-primed carbon steel's surface consisted of multi layers, which were prepared in the following step: First, the primed steel was immersed in a slurry consisting of 45wt\% PPS and 55wt\% isopropyl alcohol,

and then it was withdrawn. Second, the slurry-covered panel was left for at least twelve hours to allow the alcohol to volatize at room temperature. Finally, the panel was baked 
for 2 hours at $320^{\circ} \mathrm{C}$ to ensure the melt-flow of PPS, and then was cooled off at room temperature. This entire process, called “dipping-withdrawing-baking-cooling”, was repeated to superimpose the second coating layer of PPS slurry over the first one. Further, this process was repeated one more time to assemble a total of three layers of PPS before depositing the top coating systems containing PTFE. Afterward, a top coating layer of 100/0, 90/10, 80/20, 70/30, and 60/40 PPS/PTFE ratios was overlaid on this PPS underlying layer, using the same coating process. The composition of slurries for these PPS/PTFE blends was same as that of the single PPS, corresponding to $45 \mathrm{wt} \%$ blend power and 55wt\% alcohol. The thickness of these coatings without $\mathrm{Zn}$.Ph primer ranged from 340 to $380 \mu \mathrm{m}$.

\subsection{Results}

The self-segregating character of polytetrafluoroethylene (PTFE) from the polyphenylenesulfide (PPS) in the melt-flowing process of PTFE/PPS blend powder gave it a high potential as a slip-and water repellent-enhancing, anti-corrosion coating for carbon steel steam separators operating at brine temperature of $\sim 210^{\circ} \mathrm{C}$ in geothermal power plants. The segregated PTFE polymer occupied the outermost surface site of coating film. Correspondingly, the surface free energy of this blend polymer coating depended on the PPS/PTFE ratios; this energy significantly declined as more PPS was replaced by PTFE, from $24.3 \mathrm{mJm}^{-1}$ for $100 / 0$ PPS/PTFE ratio to $1.7 \mathrm{mJm}^{-1}$ for $60 / 40$ ratio (Figure 52). In other words, the surfaces of PTFE-rich PPS coating offered enhanced water repellency. However, PTFE underwent hydrothermal oxidation when the blend polymer coating was immersed for up to 35 days in the $\mathrm{CO}_{2}$-laden brine at $300^{\circ} \mathrm{C}$. This oxidation led to the rupture of backbone C-C linkages and C-F bonds within the PTFE, reflecting the transformation of PTFE's molecular structure into an alkylated polyfluorocarboxylate salt complex linked to Na. Such transformation not only caused the increase in kinetic coefficient of friction (KCOF) and surface free energy of the coatings, but also impaired PTFE's thermal stability, from a decomposition temperature of $481^{\circ} \mathrm{C}$ for unexposed PTFE to one of $438^{\circ} \mathrm{C}$ for 35 days of exposure. A marked increase in surface free energy occurred in the first 7 days exposure; beyond that time, it almost leveled off (Table 9). After exposure for 14 days, the surface free energy of the 
PTFE-rich PPS coatings made with the 70/30 and 60/40 ratios was $\sim 26$ and $~ 28 \%$ lower, respectively, than that of the bulk PPS without PTFE. Such changes in surface free energy were correlated directly with the changes in the KCOF value (Figure 53); the increased surface free energy corresponded to the increment of KCOF value. A very low KCOF value of 0.19 was obtained from the 70/30 and 60/40 PPS/PTFE ratios before exposure, reflecting a great slipperiness of coating's surface. This value represented a decrease of $47 \%$ and 2.6-fold compared with that of the PPS surface without Teflon and the stainless steel (SS) surface, respectively. When the Teflon-rich PPS was exposed to hot brine, the KCOF value rose by $21 \%$ to 0.23 in the first 7 days exposure; beyond that time, it seemed to level off. In contrast, the friction of SS surfaces increased from 0.5 before exposure to 0.9 after 14 days exposure, raising concerns that the enhanced surface asperity accompanying this increment in friction might promote the deposition of scales (Figure 54).

Although the increased surface free energy and KCOF raised concerns over the diminution of maximum efficacy of PTFE in enhancing the water repellency and slipperiness of the coating, the top coating layer with 70/30 and 60/40 ratios offered great protection of carbons steel against corrosion in $300^{\circ} \mathrm{C}$ brine. In fact, even though a coating consisting of an underlying PPS layer and top PPS/PTFE blend layer was exposed for 35 days to hot brine, the PTFE-rich top layer considerably reduced the rate of transportation of moisture and corrosive electrolyte through the coating (Figure 55). In contrast, bulk PPS and PTFE-poor PPS blend top coating systems failed after 14 days exposure, as shown by the presence of blisters on the coated steel panels. 


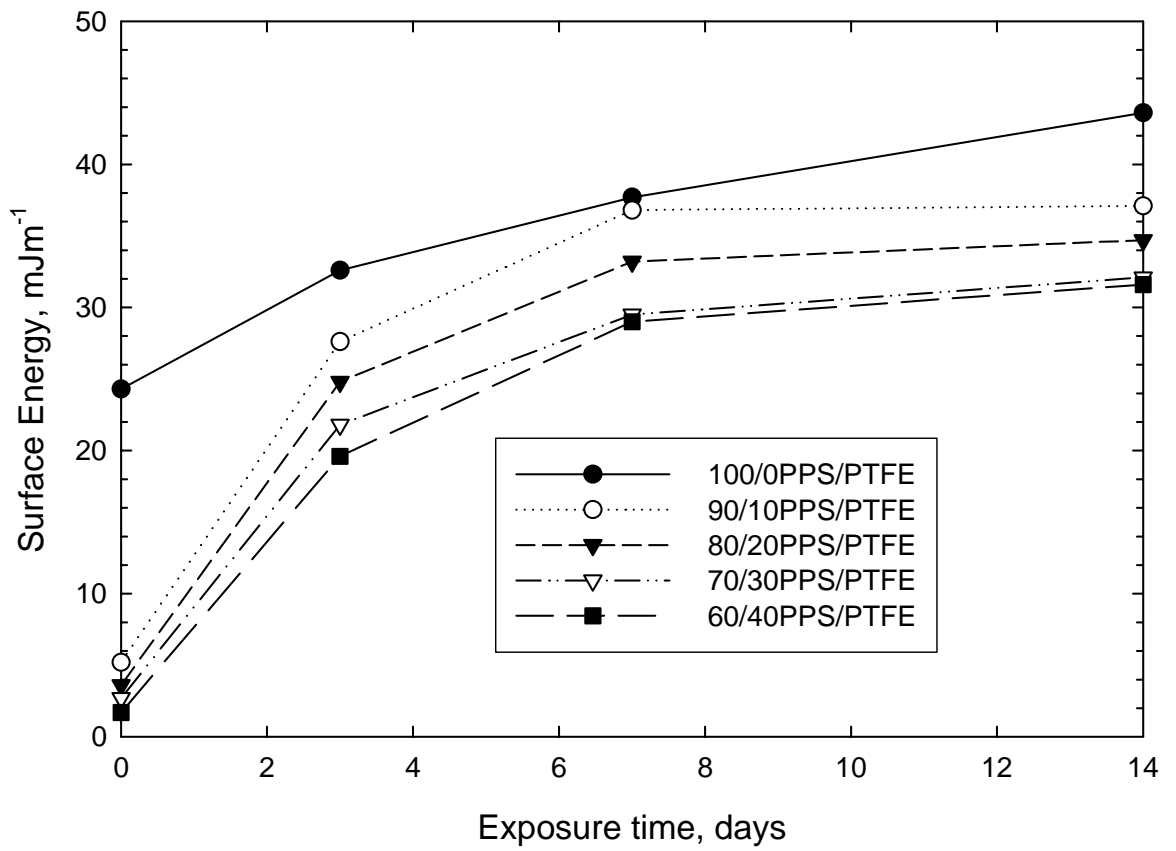

Figure 52. Changes in surface energy of 100/0, 90/10, 80/20, 70/30, and 60/40 PPS/PTFE ratio coatings as a function of exposure time. 
Table 9. Surface free energy and its components for various PPS/PTFE ratio coatings after and before exposure.

\begin{tabular}{|c|c|c|c|c|c|c|}
\hline \multirow[t]{2}{*}{ PPS/PTFE } & \multirow{2}{*}{$\begin{array}{l}\text { Exposure } \\
\text { time } \\
\text { days, }\end{array}$} & \multicolumn{2}{|c|}{$\begin{array}{l}\text { Contact angle, } \\
\text { degrees }\end{array}$} & \multirow{2}{*}{$\begin{array}{l}\text { Disperse } \\
\text { force } \\
\text { component, } \\
\gamma_{\mathrm{s}}^{\mathrm{d}}, \mathrm{mJm}^{-1}\end{array}$} & \multirow{2}{*}{$\begin{array}{l}\text { Polar force } \\
\text { component, } \\
\gamma_{\mathrm{s}}^{\mathbf{p}}, \mathrm{mJm}^{-1}\end{array}$} & \multirow{2}{*}{$\begin{array}{l}\text { Surface } \\
\text { free } \\
\text { energy, } \\
\gamma_{\mathrm{s}, \mathrm{mJm}}{ }^{-1}\end{array}$} \\
\hline & & Water & Glycerol & & & \\
\hline \multirow[t]{4}{*}{$100 / 0$} & 0 & 90.7 & 76.4 & 15.7 & 8.6 & 24.3 \\
\hline & 3 & 71.1 & 72.5 & 5.8 & 26.8 & 32.6 \\
\hline & 7 & 64.9 & 66.6 & 6.6 & 31.1 & 37.7 \\
\hline & 14 & 59.9 & 64.7 & 4.7 & 38.9 & 43.6 \\
\hline \multirow[t]{4}{*}{$90 / 10$} & 0 & 118.1 & 115.6 & 2.3 & 2.9 & 5.2 \\
\hline & 3 & 77.3 & 78.6 & 4.9 & 22.7 & 27.6 \\
\hline & 7 & 70.0 & 76.5 & 2.1 & 34.7 & 36.8 \\
\hline & 14 & 66.6 & 70.0 & 4.8 & 32.3 & 37.1 \\
\hline \multirow[t]{4}{*}{$80 / 20$} & 0 & 124.8 & 121.7 & 1.9 & 1.7 & 3.6 \\
\hline & 3 & 80.6 & 80.6 & 5.5 & 19.3 & 24.8 \\
\hline & 7 & 73.2 & 78.6 & 2.4 & 30.8 & 33.2 \\
\hline & 14 & 73.1 & 80.0 & 1.6 & 33.1 & 34.7 \\
\hline \multirow[t]{4}{*}{$70 / 30$} & 0 & 129.5 & 125.9 & 1.6 & 1.1 & 2.7 \\
\hline & 3 & 84.6 & 82.6 & 6.8 & 15.0 & 21.8 \\
\hline & 7 & 77.3 & 81.8 & 2.5 & 27.0 & 29.5 \\
\hline & 14 & 75.3 & 81.2 & 1.9 & 30.3 & 32.1 \\
\hline \multirow[t]{4}{*}{$60 / 40$} & 0 & 134.4 & 132.1 & 0.9 & 0.8 & 1.7 \\
\hline & 3 & 88.2 & 84.6 & 8.0 & 11.6 & 19.6 \\
\hline & 7 & 77.3 & 84.3 & 2.9 & 26.1 & 29.0 \\
\hline & 14 & 77.3 & 84.4 & 1.1 & 30.5 & 31.6 \\
\hline
\end{tabular}




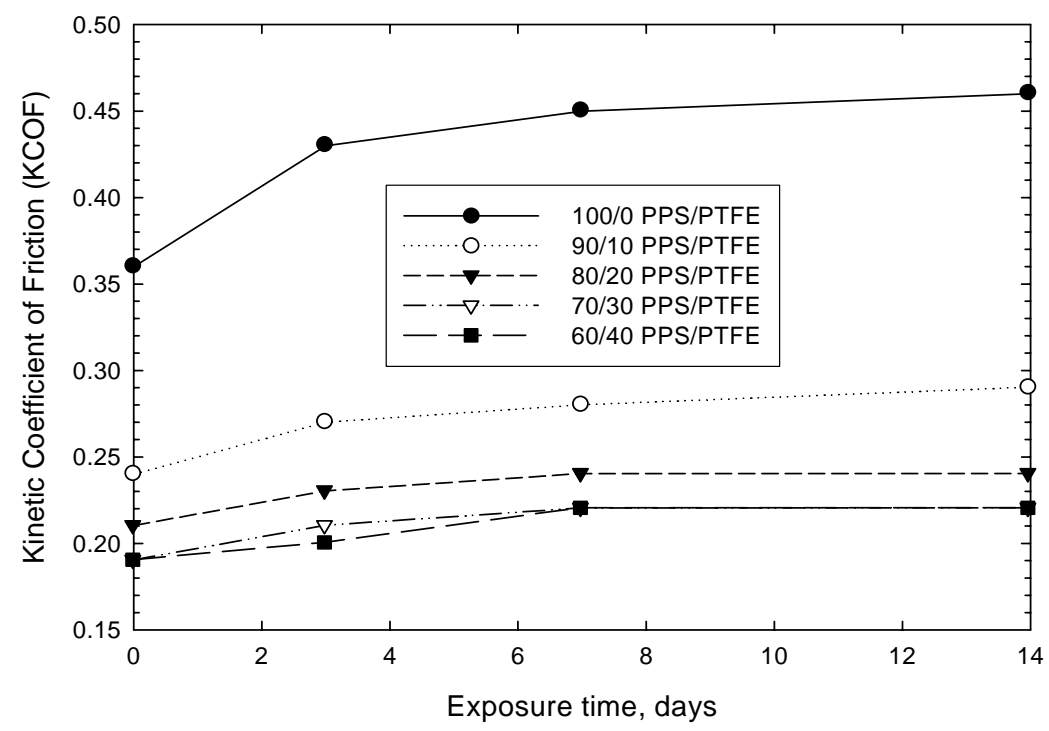

Figure 53. Changes in KCOF value of 100/0, 90/10, 80/20, 70/30, and 60/40 PPS/PTFE ratio coatings as a function of exposure time.

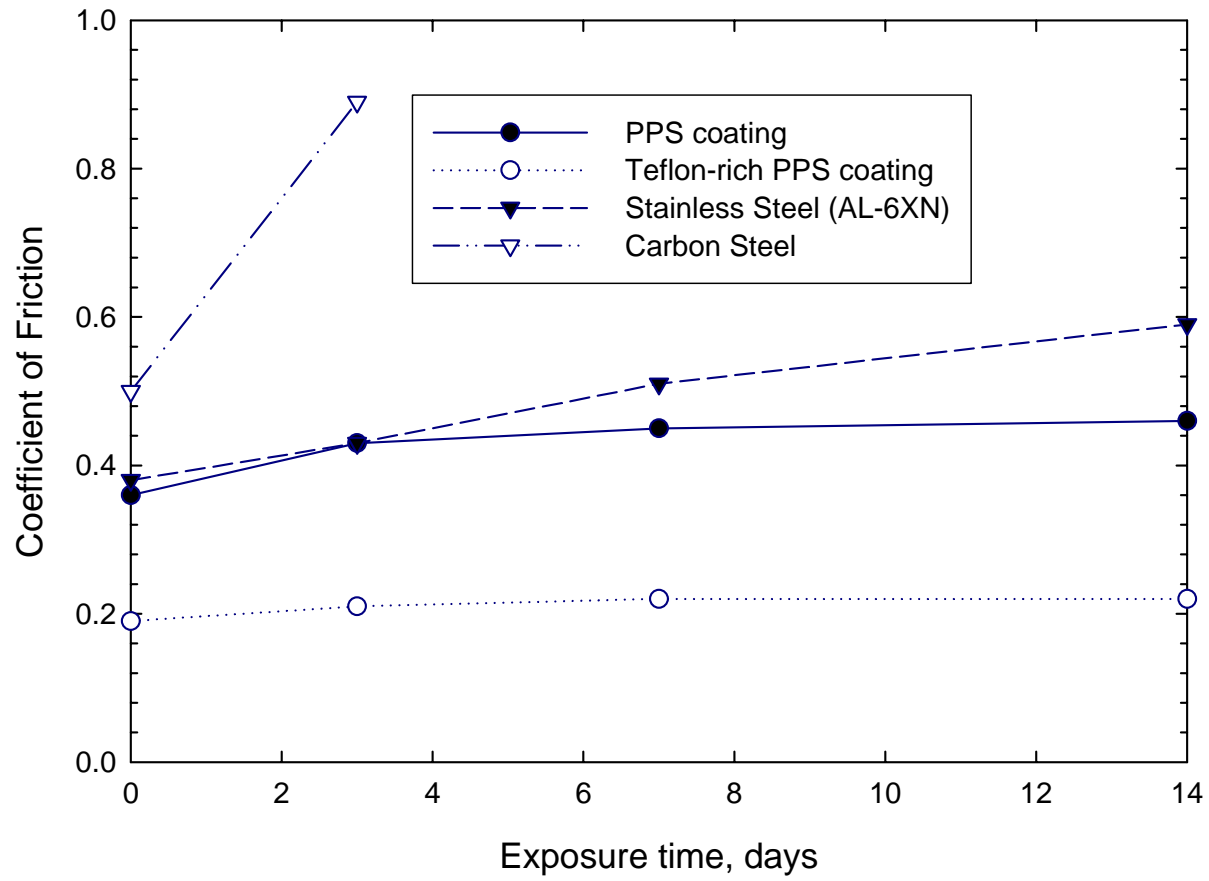

Figure 54. Comparison of KCOF values of PPS, Teflon-rich PPS (60/40 PPS/PTFE ratio), stainless steel, and carbon steel surfaces before and after exposure. 


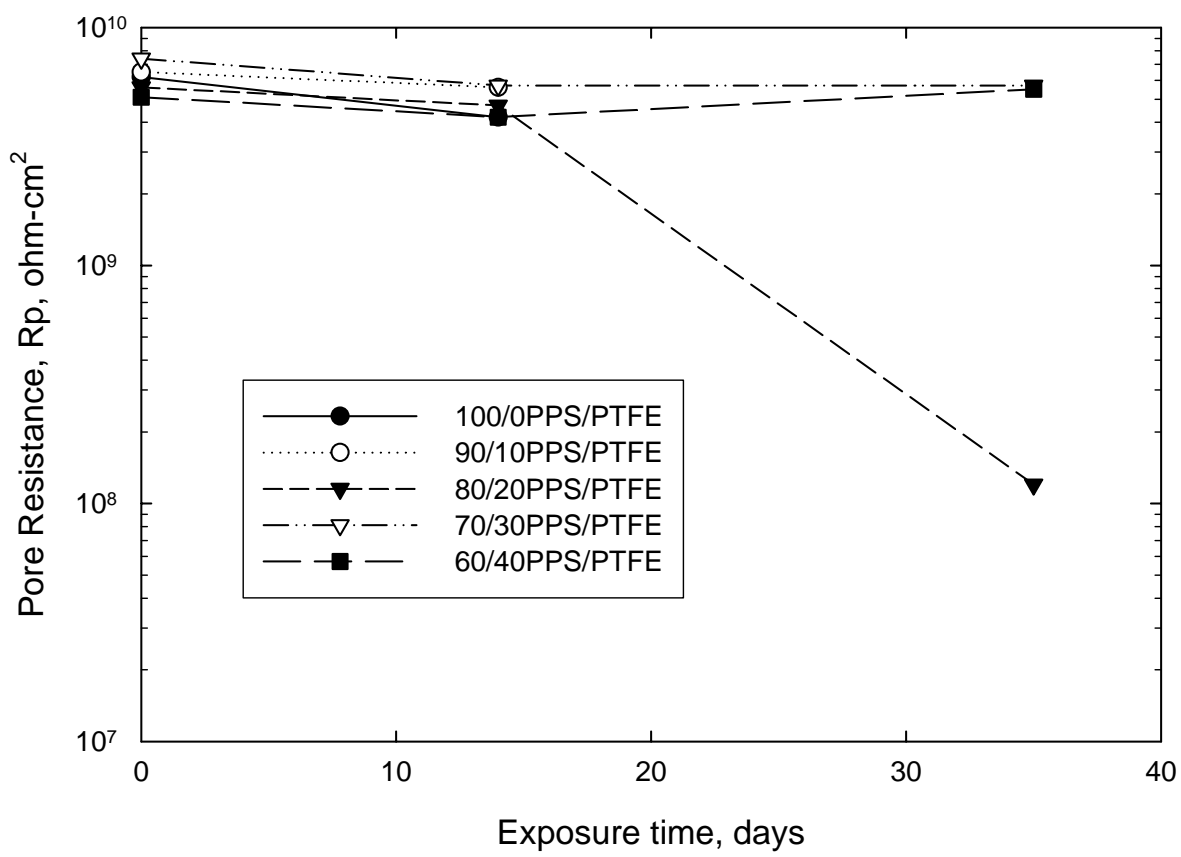

Figure 55. Changes in pore resistance, Rp, of 100/0, 90/10, 80/20, 70/30 and 60/40 PPS/PTFE ratio coatings as a function of exposure time.

\subsection{Conclusions}

The superficial layer of the assembled coating was occupied by PTFE selfsegregated from PPS during the melt-flowing process of this blend polymer; it conferred an outstanding slipperiness and water repellent properties because of its low friction and surface free energy. However, PTFE underwent hydrothermal oxidation in hot brine, transforming its molecular structure into an alkylated polyfluorocarboxylate salt complex linked to Na. Although such molecular transformation increased the friction and surface free energy, and also impaired the thermal stability of PTFE, the top PTFE-rich PPS layer significantly contributed to preventing the permeation of moisture and corrosive electrolytes through the coating film, so mitigating the corrosion of carbon steel.

\subsection{Publication}


- Sugama, T. and Jung D., "Polytetrafluoroethylene-rich Polyphenylenesulfide Blend Top Coatings for Mitigating Corrosion of Carbon Steel in $300^{\circ} \mathrm{C}$ Brine”, Int. J. Prog. in Organic Coatings, (submitted).

\section{Impact of Work}

Nanocomposite and surface processing technologies developed in this project led to the creation of the upgraded coating systems with five significantly advanced properties, 1) the increase in coating's hydrothermal stability to $>250^{\circ} \mathrm{C}, 2$ ) the considerable reduction of hydrothermal oxidation, 3) the extension of useful lifetime of aluminum and carbon steel to more than 1000 hours in very corrosive environment, 4) the generation of very low friction surface, and 5) the mitigation of scale depositions. In addition, new hydrothermally self-improving hybrid coatings provided the likelihood as a low-temperature curable corrosion-preventing coating for the joint areas. Since these innovative coating technologies are aimed at protecting the inexpensive carbon steel- and aluminum-based plant components against corrosion and fouling, contingent upon their scale-up success, they will not only result in a considerable reduction of the needed capital investment, but also decrease the costs of operations and maintenance, contributing to geothermal technologies goal of nearly $20 \%$ reduction of power plant economic factors in 2010. 


\section{References}

[1] C.F. Kutscher and D. Dostenaro, Geothermal Ressour. Counc. Trans., 26, (2002) 775.

[2] R.W. Lenz, C.E. Handlovity, and H.A. Smith, J. Poly. Sci. 58, (1961) 351.

[3] P.C. Dawson and D.J. Blundell, Polymer, 21, (1980) 577.

[4] A.J. Waddon, M.J. Hill, A. Keller, and D.J. Blundell, J. Mater. Sci. 22, (1987) 1173.

[5] K.S. Lenz, Chemical \& Engineering News, 71, (1999) 35.

[6] C.R. Shastry and H.E. Townsend, Corrosion, 45, (1989) 103.

[7] H. Jr. Leidheiser and W. Wang, “Corrosion Control by Organic Coatings”, H. Jr. Leidheiser (Ed.), National Association of Corrosion Engineers, pp 70-77, Houston, TX, 1981.

[8] R.A. Dickie, “Adhesion Aspects of Polymeric Coatings”, K.L. Mittla (Ed.), Plenum Press, pp 319-327, New York, 1983.

[9] J.S. Hammond, J.W. Holubka, J.E. DeVries, and R.A. Dickie, Corro. Sci., 21, (1984) 239.

[10] J.F. Watts and J.E. Castle, J. Mater. Sci., 19, (1981) 239.

[11] R.F. Hamadeh, D.A. Dillard, K.M. Liechti, and J.S. Thomton, J. Adhesion Sci. Tech. $\underline{3}$, (1989) 421.

[12] R. Sard, Plat, Surf. Finish, 74, (1987) 30.

[13] T. Sugama, L.E. Kukacka, N. Carciello, and J.B. Warren, J. Appl. Polym. Sci., 30, (1985) 4357.

[14] T. Sugama and N. Carciello, Int. J. Adhesion and Adhesives, 12, (1992) 27.

[15] T. Sugama and K. Gawlik, Polymers \& Polymer Comp., 9 9, (2001) 377.

[16] T. Sugama and N. Carciello, J. Coat. Tech., 66, (1994) 43.

[17] A. Tamashusky, “Carbon fiber guide”, Asbury Graphite Mills, Inc., Report, February 2000.

[18] F. Mansfield, M.N. Kending, and S. Tsai, Corrosion, 38, (1982) 570.

[19] D.W. Dwight and W.M. Riggs, J. Colloid and Interface Sci., 47, (1974) 650.

[20] D.M. Roy, Z. Nadagawa, B.E. Scheetz, E.L. White, in: J.F. Young (Ed.), Very High Strength Cement-based Materials, Mater. Res. Soc., 42, (1985) 245. 
[21] P.K. Mehta, Cem. Concr. Res., $\underline{6}$, (1976) 169.

[22] J.F. J.F. MacDowell, in: B.E. Scheetz, A.G.Landers, I. Odler and H. Jennings (Eds), Specialty Cements with Advanced Properties, Materials Research Society, 179 (1991) 159.

[23] G.G. Wicks, A.R.A. Londding and M.A. Molecke, Mater. Res. Bull, 18 (1993) 32.

[24] P.E. Cassidy, in: L.Lee (Ed.), Adhesive, Sealants, and Coatings for Space and Harsh Environments, Plenum Press, New York, 1987, p. 187.

[25] M. Share, Chemical \& Engineering News, 79 (2001) 40.

[26] M. Litt, R. Ameri, Y. Wang, R. Savinell and J. Wainright, Mater. Res. Soc. Symp. Proc., 548 (1999) 313.

[27] P. Staiti and M. Minutoli, J. Power Socurces, 94 (2001) 9.

[28] D.J. Jones and J. Roziere, J. Membrane Sci., 185 (2001) 41.

[29] A. Schechter and R.F. Savinell, Solid State Ionics, 147 (2002) 181.

[30] T.H. Lee and J.W. J. Electron Spectrosc. Relat. Phenom., 11 (1977) 112.

[31] H. Ulrich, "Introduction to Industrial Polymers", Palmertone Publishing Corp., New York, 1974, p. 153.

[32] J. Peeling, J. Appl. Polym. Sci. 26 (1981) 3761.

[33] L.H. Perng, Polym. Degrad. Stab. 69 (2000) 323.

[34] T. Sugama, L.E. Kukacka, and W. Horn, "Hydrothermal Stability of Vinyl-Type Polymer Concrete Containing Tricalcium Silicate”, Cem. Conc. Res., 9, (1979) 461-471.

[35] T. Sugama, L. E. Kukacka and W. Horn, "Hydraulic Cement-type Fillers for Hydrotheramlly Stable Polymer Concretes”, J. Mater. Sci., 15, (1980) 1498-1508.

[36] T. Sugama, L. E. Kukacka and W. Horn, "Hydrothermal Durability of Capolyester Complexed Polymer Concrete”, Cem. Conc. Res., 11, (1981) 429-442.

[37] T.E. Hale, in Ceramic Films and Coatings, J.B. Wachtman and R.A. Haber [Eds], Noyes Publication, New Jersey, p.22, (1993).

[38] R.S. Bonetti, H. Wiperachtiger, and E. Mohn, Metal Powder Report, 26 (1990) 657.

[39] S. Sampath, G.A. Banke, H. Herman and S. Rangaswamy, Surf. Eng. 5 (1989) 293. 
[40] D.N. Ronald, R.B. Randy and M.V. Nathal "Physical and Mechanical Metallurgy of NiAl”, National Aeronautics and Space Administration (NASA) Technical Paper 3398, April (1994).

[41] T. Sugama, K. Gawlik, Polym. Polym. Compos. 11 (2003) 161.

[42] Y. Komori, K. Kuroda, in: T.J. Pinnavaia and G.W. Beall (Eds.), Polymer-Clay Nanocomposites, John Wiley \& Sons, Ltd., New York, 2000, pp. 3-18.

[43] E. Ruiz-Hitzky, P. Aranda, in: T.J. Pinnavaia and G.W. Beall (Eds.), PolymerClay Nanocomposites, John Wiley \& Sons, Ltd., New York, 2000, pp. 18-46.

[44] C. Chen, D. Curliss, Polym. Materi. Sci. \& Eng. 88 (2002) 88.

[45] A. Goldstein, M. Beer, J. Euro. Ceram. Soc. 24 (2004) 3187.

[46] J.Y. Lee, H.K. Lee, Mater. Chem. Phys. 85 (2004) 410.

[47] M. Pospisil, A. Kalendova, P. Capkova, J. Simonik, M. Valaskova, Colloid Interface Sci. 277 (2004) 154.

[48] A.B. Morgan, J.W. Gilman, R.H. Harris, C.L. Jackson, C.A. Wilkie, J. Zhu, Polym. Materi. Sci. \& Eng. 83 (2000) 53.

[49] A. Tidjani, C.A. Wilkie, Polym. Degrad. Stab., 74 (2001) 33.

[50] B. Jurkowski, Y.A. Olkhov, Thermochimica Acta, 414 (2004) 243.

[51] C. Lam, H. Cheung, K. Lau, L. Zhou, M. Ho, D. Hui, Composites: Part B, 36 (2005) 263.

[52] T. Sugama, L.E. Kukacka, and N. Carciello, Prog. Org. Coat., 18 (1990) 173.

[53] F. Mansfeld, C.B. Breslin, A. Pardo, and F.J. Perez, Surf. Coat. Tech., 90, (1997) 224.

[54] A. Aballe, M. Bethencourt, F.J. Botana, and M. Marcos, J. Alloys and Compounds, 323, (2001) 855.

[55] M. Dabala, L. Armelao, A. Buchberger, and I. Calliari, Appl. Surf. Sci., 172, (2001) 312.

[56] M. Forsyth, C.M. Forsyth, K. Wilson, T. Behrshing, and G.B. Deacon, Corros. Sci., 44, (2002) 2651. 
[57] W. Fahrenholtz, M.J. O’Keefe, H. Zhou, and J.T. Grant, Surf. Coat. Tech., 155, (2002) 208 .

[58] X. Yu and C. Cao, Thin Solid Films, 423, (2003) 252.

[59] T. Sugama, Polym. Polym. Comp. 6 (1998) 373.

[60] Y. Ikada and Y. Uyama, "Lubricating polymer surfaces”, Technomic Publishing Co., Lancaster, PA, USA, (1993) pp. 41-54. 